\title{
An Archaeological Survey of Twin Buttes Reservoir, Tom Green County, Texas, Volume III
}

\author{
Raymond P. Mauldin \\ Center for Archeological Research, University of Texas at San Antonio \\ David L. Nickels
}

Follow this and additional works at: https://scholarworks.sfasu.edu/ita

Part of the American Material Culture Commons, Archaeological Anthropology Commons, Environmental Studies Commons, Other American Studies Commons, Other Arts and Humanities Commons, Other History of Art, Architecture, and Archaeology Commons, and the United States History Commons

Tell us how this article helped you.

This Article is brought to you for free and open access by the Center for Regional Heritage Research at SFA ScholarWorks. It has been accepted for inclusion in Index of Texas Archaeology: Open Access Gray Literature from the Lone Star State by an authorized editor of SFA ScholarWorks. For more information, please contact cdsscholarworks@sfasu.edu. 


\section{An Archaeological Survey of Twin Buttes Reservoir, Tom Green County, Texas, Volume III}

\section{Creative Commons License}

\section{(c) (1) \&}

This work is licensed under a Creative Commons Attribution-NonCommercial 4.0 International License 


\section{An Arehaedogical Suiver of Iwin Buttes Reservoir t. If Tom-Greeg County, Texas}

\section{Volume III}

- M. ARavmond P. Mauldin

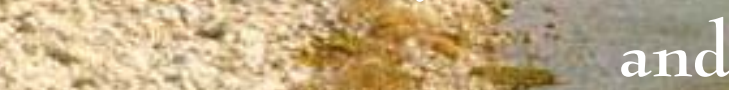 \\ David I. Nickels}

with Contributions by

C. Britt Bousman, Wulf Gose, Ruth Mathews, Preston McWhorter, Kristi Miller, Clemente Murguia, Lee Nordt, Tanya Norris, Katie Plum, Rick Robinson, and Preston Stone

Center for Archaeological Research The University of Texas at San Antonio Archaeological Survey Report, No. 300 2001
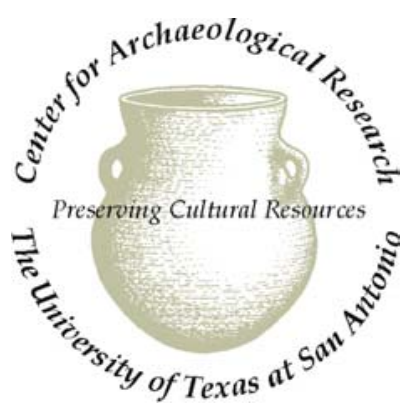

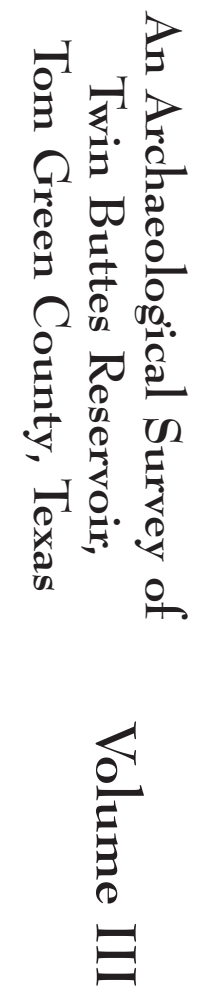

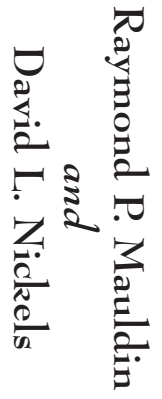

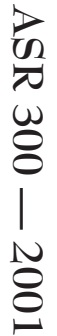




\title{
An Archaeological Survey of Twin Buttes Reservoir, Tom Green County, Texas
}

\author{
Volume III
}

\section{Raymond P. Mauldin and David L. Nickels}

\author{
Robert J. Hard and C. Britt Bousman \\ Principal Investigators
}

Ccopyright 2001

Center for Archaeological Research

The University of Texas at San Antonio

Archaeological Survey Report, No. 300 
The following information is provided in accordance with the General Rules of Practice and Procedure, Chapter 41.11 (Investigative Reports), Texas Antiquities Committee:

1. Type of investigation: Class III archaeological survey

2. Project name: Twin Buttes Archaeological Survey

3. County: Tom Green

4. Principal investigator(s): Robert J. Hard and C. Britt Bousman

5. Name and location of sponsoring agency: Bureau of Reclamation, Oklahoma City Field Office, 4149 Highline Blvd., Suite 200, Oklahoma City, OK 73108

6. Texas Antiquities Permit No.: N/A

7. Published by the Center for Archaeological Research, The University of Texas at San Antonio, 6900 N. Loop 1604 W., San Antonio, Texas 78249-0658, 2001

A list of publications offered by the Center for Archaeological Research is available. Call (210) 458-4378; write to the Center for Archaeological Research, The University of Texas at San Antonio, 6900 N. Loop 1604 W., San Antonio, Texas 78249-0658; e-mail to car@lonestar.utsa.edu; or visit CAR's web site at http://car.utsa.edu. 


\title{
An Archaeological Survey of Twin Buttes Reservoir Tom Green County, Texas
}

\author{
Volume I: \\ Chapter 1: Introduction \\ Chapter 2: Project Overview \\ Chapter 3: Project Environment \\ Chapter 4: Geoarchaeology \\ Chapter 5: Cultural Chronology and Previous Archaeology \\ Chapter 6: Methods
}

Chapter 7: Prehistoric Data; Research Perspective, Analytical Issues, and Project Goals

Chapter 8: Survey Results and Prehistoric Data Analysis

Chapter 9: Archaeological Record Investigations

Chapter 10: Historic Sites; Background and Research Design

Chapter 11: Historic Sites; Survey Results and Analysis

Chapter 12: Summary and Recommendations

\section{Volume II:}

Appendix A: Prehistoric Site Descriptions and Summary

\section{Volume III:}

Appendix B: Shovel and Auger Data

Appendix C: Excavation Summary

Appendix D: Surface Observation Data

Appendix E: Biface Data

Appendix F: Unique Items Data

Appendix G: Isolated Finds Data

Appendix H: Radiocarbon Data

Appendix I: Soil-Stratigraphic Descriptions

Appendix J: Paleomagnetic and Magnetic Soil Susceptibility Results

Appendix K: Project Forms

Appendix L: Previous Land Ownership 


\section{An Archaeological Survey of Twin Buttes Reservoir}

\section{Contents \\ Volume III}

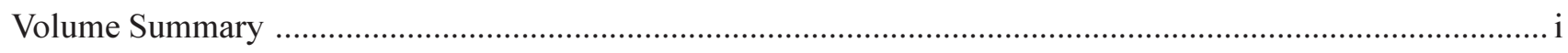

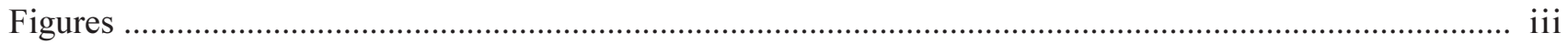

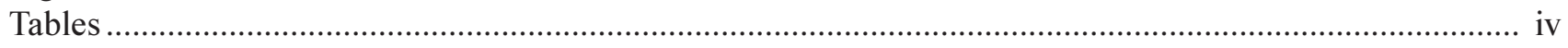

Appendix B

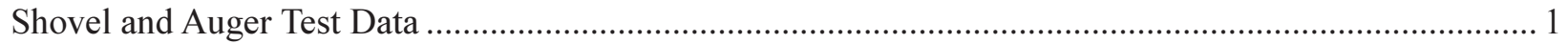

Appendix C

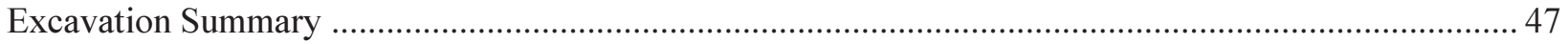

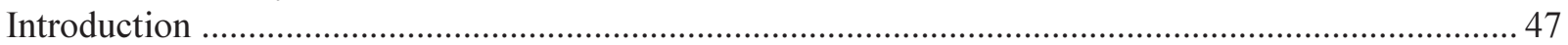

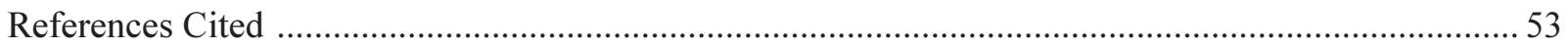

Appendix D

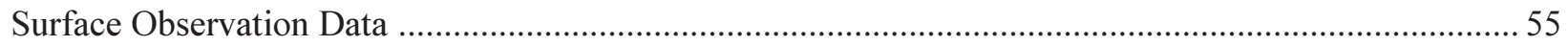

Appendix E

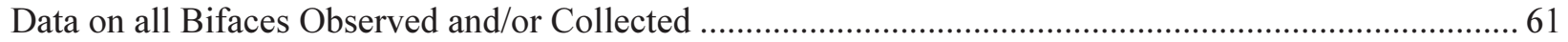

Appendix F

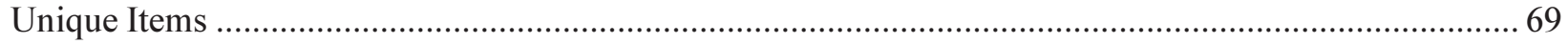

Appendix G

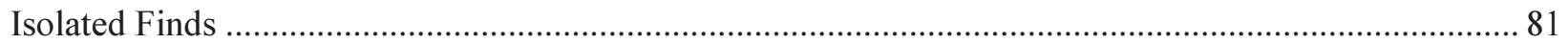

Appendix $\mathbf{H}$

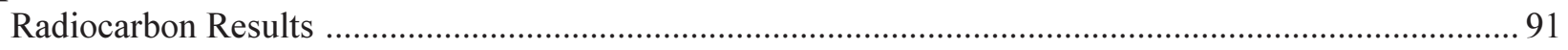

Appendix I

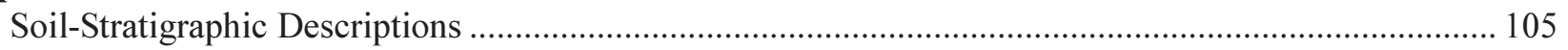

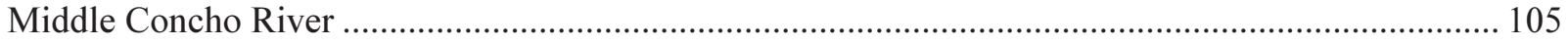

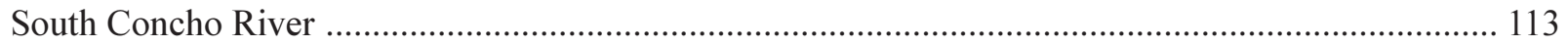

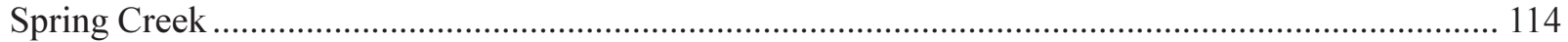

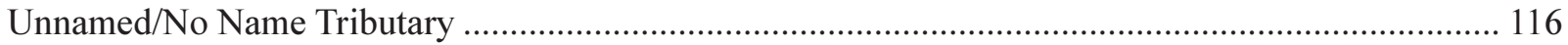

Appendix J

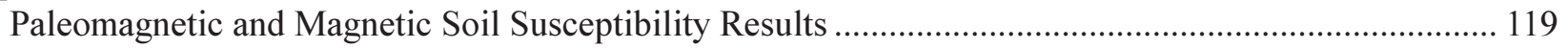

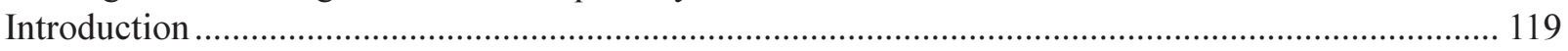

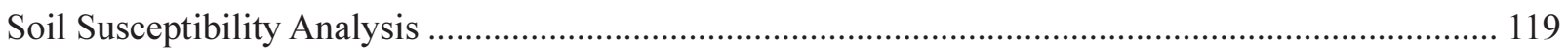

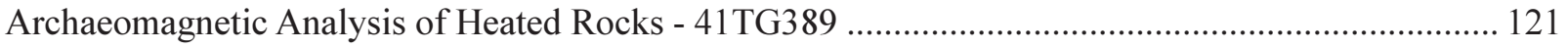

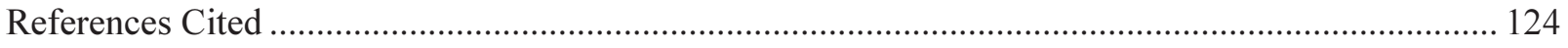

Appendix K

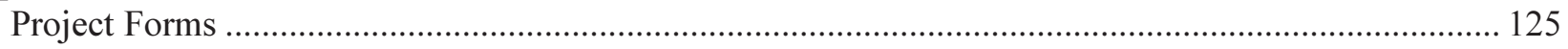

Appendix L

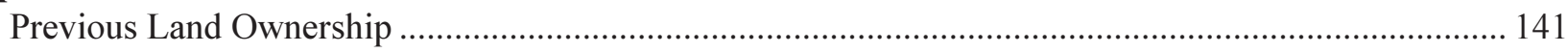




\section{Figures}

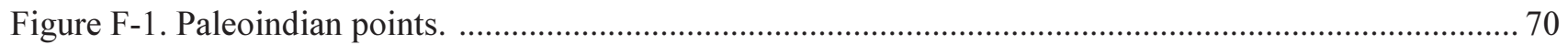

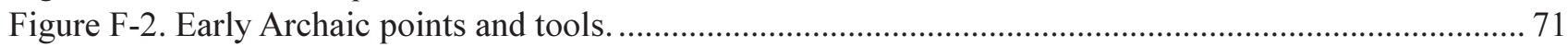

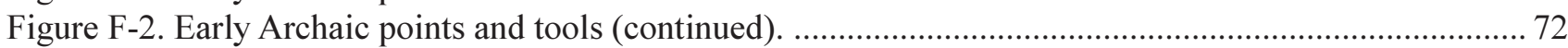

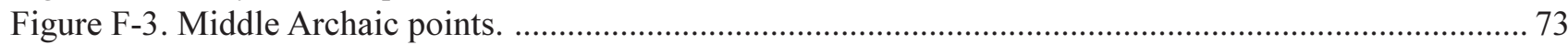

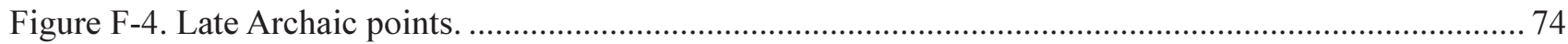

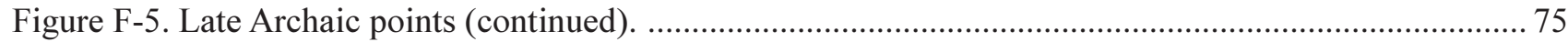

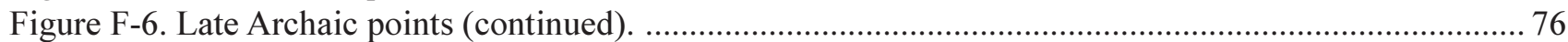

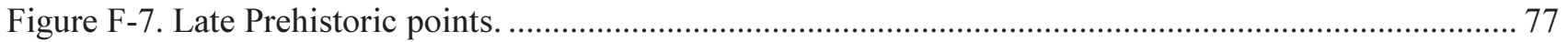

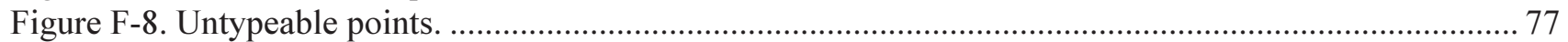

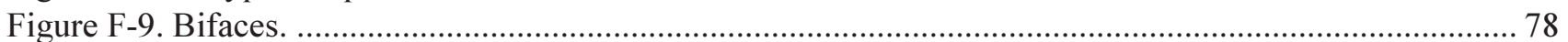

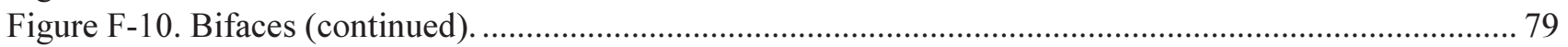

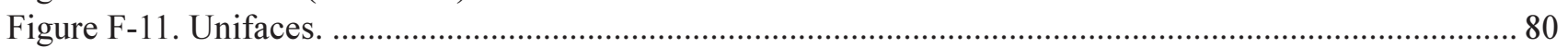

Figure G-1. Distribution of Isolated Finds recorded during Twin Buttes Archaeological Project. .................. 90

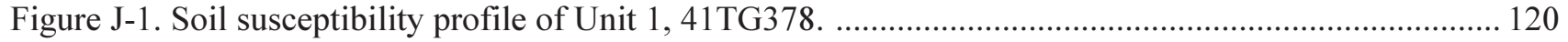

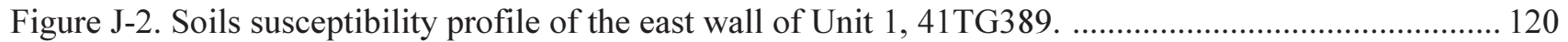

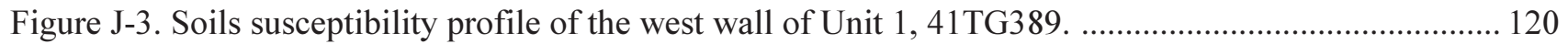

Figure J-4. Soil susceptibility profile of Unit 1, 41TG410. ............................................................ 121

Figure J-5. Components of magnetization in vector component diagrams for rocks J, E, and H. ............... 122

Figure J-6. Directions of the best fitting vectors in a stereographic projection. ......................................... 123

Figure L-1. Survey \#1896 (41TG160), Surveyor's Record, Book 1, Tom Green County............................. 156

Figure L-2. Survey \#1815 (41TG248), Surveyor's Record, Book 1, page 214, Tom Green County. ............ 157

Figure L-3. Survey \#656 (41TG253), Surveyor's Record, Book 2, page 9. .............................................. 158

Figure L-4. Survey \#876 (41TG344 and 41TG459), Surveyor's Record, Book 2, page 315. ..................... 159

Figure L-5. Survey \#824 (41TG419), Surveyor's Record, Book 2, page 311 .......................................... 160

Figure L-6. Survey \#104 (41TG445), Surveyor's Record, Book 1, page 479, Tom Green County. ............... 161

Figure L-7. Survey \#653 (41TG450), Surveyor's Record, School Land E, page 394. ................................ 162

Figure L-8. Survey \#654 (41TG452), Surveyor's Record, Book 2, page 7. ................................................. 163

Figure L-9. Survey \#875 (41TG458), Surveyor's Record, Book 2, page 316. ......................................... 164

Figure L-10. Survey \#817 (41TG501), Surveyor's Record.................................................................. 165

Figure L-11. Survey \#888 (41TG504), Surveyor's Record. .................................................................. 166

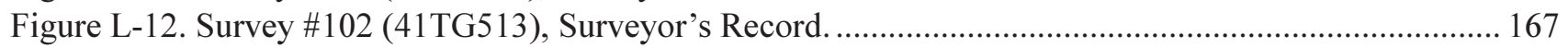

Figure L-13. Survey \#814 (41TG516), Surveyor's Record, Book 2, page 341. .......................................... 168

Figure L-14. Survey \#647 (41TG520, 41TG521, and 41TG523), Surveyor's Record, Book 2, page 340.... 169 


\section{Tables}

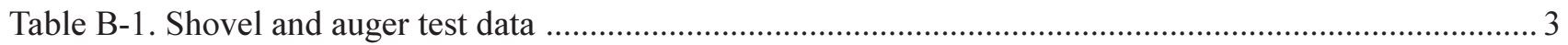

Table C-1. Vertical distribution of flakes and soils from 41TG389 ......................................................... 49

Table C-2. Attributes of flakes recovered from 41TG410 .................................................................. 51

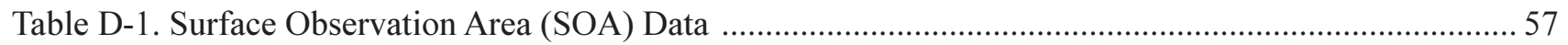

Table E-1. Data on bifaces observed and/or collected during the Twin Buttes survey ................................... 62

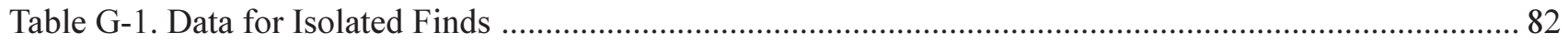

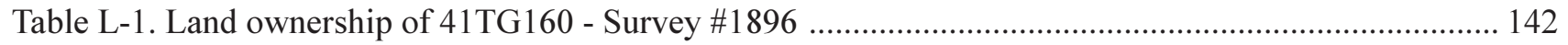

Table L-2. Land ownership of 41TG248 - Survey \#958 and Survey \#1815 …......................................... 142

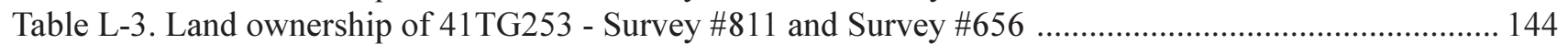

Table L-4. Land ownership of 41TG344 and 41TG459 - Survey \#876 ...................................................... 145

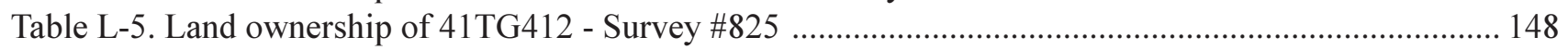

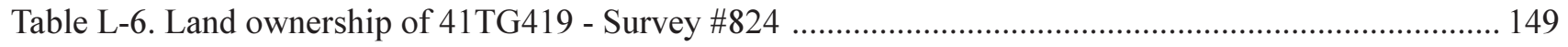

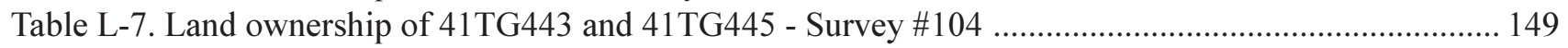

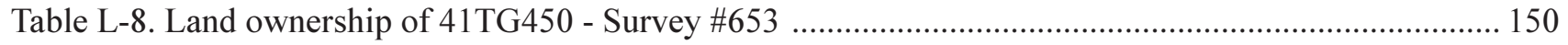

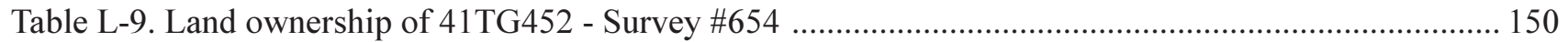

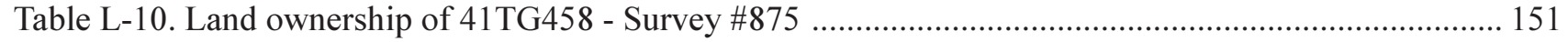

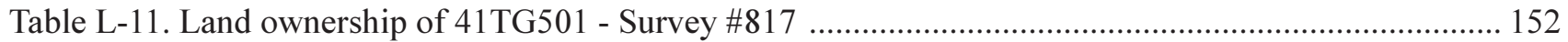

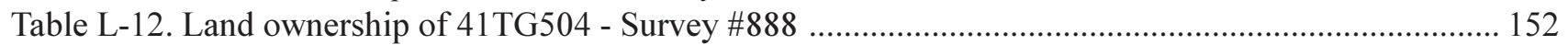

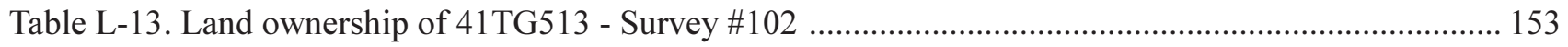

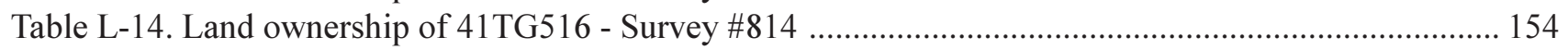

Table L-15. Land ownership of 41TG520, 41TG521, 41TG523 - Survey \#647 …...................................... 155 


\title{
Appendix B: \\ Twin Buttes Archaeological Project
}

\author{
Shovel and Auger Test Data
}

The following definitions, explanations and codes were used during the analysis of cultural material recovered from shovel and auger tests:

\section{Artifact Type}

1. Flakes. All utilized flakes and/or debris which cannot be classified as a core, tool, or ground stone. Flakes and debris do not necessarily have to have a clearly distinguishable ventral and dorsal surface - pieces often referred to as "chunks" or "angular debris" are of this type.

2. Cores. Cores are defined as any parent block from which flakes have been removed. This category does not, however, include tools. Cores must have at least three negative bulbs and cannot have a positive bulb; if a positive bulb is present in this classification, it is a flake and not a core. "Flake cores" were not a category for the purposes of this analysis.

3. Tested Cobbles. A tested cobble must have one or two negative bulbs present; no positive bulb.

4. Bifaces. This type includes any item on which both faces have flake scars that cover at least $1 / 3$ of the face and have been removed in a systematic manner. This class includes points, preforms, and blanks. Pieces elsewhere called "bifacial cores" were considered as cores for the purposes of this analysis, and functional use was not considered.

5. Unifaces. This type includes any item on which only one face has systematically removed flakes that cover at least $1 / 3$ of that face. In some cases, unifacial retouch may be accompanied by use and retouch; in this case the piece was still classified as a uniface.

6. Retouched/Utilized Flakes. This type includes any item on which flakes have been systematically removed from an edge, but which does not qualify as a biface or uniface.

7. Other. This includes any specimen not covered above such as ground stone, hammerstone, etc. Their identity would be specified in the comments section.

\section{Maximum Length}

This is the maximum length of an artifact, recorded in $1-\mathrm{cm}$ units. All artifacts are subject to this category ( 0 to $1 \mathrm{~cm}=1 ; 1$ to $2 \mathrm{~cm}=2 ; 2$ to $3 \mathrm{~cm}=3 \ldots$...tc.). 


\section{Maximum Thickness}

This is the maximum thickness of an artifact, recorded in 1-cm units as shown above for Maximum Length.

\section{$\underline{\text { Cortex }}$}

This is the percentage of cortex on the dorsal surface (including the platform) of all items with the exception of cores, tested cobbles, and the "other" artifact type (see notes below). The following groupings were used:

$\begin{array}{ll}0 \%=\text { No Cortex } & \text { Code as } 0 \\ 1-25 \% \text { Cortex } & \text { Code as } 12 \\ 26-50 \% \text { Cortex } & \text { Code as } 38 \\ 51-75 \% \text { Cortex } & \text { Code as } 62 \\ 76-99 \% \text { Cortex } & \text { Code as } 88 \\ 100 \% \text { Cortex } & \text { Code as } 100\end{array}$

On Cores, tested cobbles, and "other" types the following codes were used:

$\begin{array}{ll}\text { No Cortex } & \text { Code as 0 } \\ \text { Less than 51\% Cortex } & \text { Code as 25 } \\ \text { More than 50\% Cortex } & \text { Code as 75 }\end{array}$

\section{Material Color}

The vast majority of the material was coded as 1, which is either gray, brown, or blue-gray. Codes 2 and 4 are white and/or pale white. Code 3 is either pinkish or red; often heat-treated. Code 9 is any other color.

\section{Fire Cracked Rock}

If present, the type of rock is noted.

\section{Mussel Shell}

Only presence or absence is noted.

\section{Other/Remarks}

The presence or absence of other cultural material, and/or remarks is noted. 
Table B-1. Shovel and auger test data

\begin{tabular}{|c|c|c|c|c|c|c|c|c|c|c|}
\hline $\begin{array}{r}\text { Site } \\
41 \mathrm{TG}\end{array}$ & $\begin{array}{l}\text { Shovel } \\
\text { Test\# }\end{array}$ & $\begin{array}{c}\text { Depth } \\
\text { cm }\end{array}$ & $\begin{array}{c}\text { Artifact } \\
\text { Type }\end{array}$ & $\begin{array}{l}\text { Max. } \\
\text { Length }\end{array}$ & $\begin{array}{c}\text { Max. } \\
\text { Thickness }\end{array}$ & $\begin{array}{c}\text { Cortex } \\
\% \\
\end{array}$ & $\begin{array}{c}\text { Material } \\
\text { Color }\end{array}$ & $\begin{array}{c}\text { Fire Cracked } \\
\text { Rock }\end{array}$ & $\begin{array}{c}\text { Mussel } \\
\text { Shell }\end{array}$ & $\begin{array}{c}\text { Other / } \\
\text { Remarks }\end{array}$ \\
\hline 106 & 1 & $0-10$ & 1 & 3 & 1 & 0 & 1 & & & \\
\hline 106 & 1 & $0-10$ & 1 & 1 & 1 & 0 & 1 & & & \\
\hline 106 & 1 & $0-10$ & 1 & 3 & 1 & 12 & 1 & & & \\
\hline 106 & 1 & $40-50$ & 6 & 2 & 1 & 12 & 1 & & & \\
\hline 106 & 1 & $40-50$ & 1 & 4 & 1 & 0 & 1 & & & \\
\hline 106 & 1 & $40-50$ & 1 & 2 & 1 & 12 & 1 & & & \\
\hline 106 & 1 & $40-50$ & 1 & 2 & 1 & 12 & 1 & & & \\
\hline 106 & 1 & $40-50$ & 1 & 2 & 1 & 0 & 1 & & & \\
\hline 106 & 1 & $50-60$ & 1 & 2 & 1 & 12 & 1 & & & \\
\hline 106 & 1 & 50-60 & 6 & 4 & 1 & 12 & 1 & & & \\
\hline 106 & 2 & $0-10$ & 2 & 4 & 2 & 0 & 1 & & & \\
\hline 106 & 2 & $0-10$ & 1 & 2 & 1 & 0 & 1 & & & \\
\hline 106 & 2 & $0-10$ & 6 & 4 & 1 & 38 & 1 & & & \\
\hline 106 & 2 & $0-10$ & 1 & 2 & 1 & 0 & 1 & & & \\
\hline 106 & 2 & $0-10$ & 1 & 2 & 1 & 0 & 1 & & & \\
\hline 106 & 2 & $0-10$ & 1 & 2 & 1 & 0 & 1 & & & \\
\hline 106 & 2 & $0-10$ & 1 & 2 & 1 & 0 & 3 & & & \\
\hline 106 & 2 & $10-20$ & 2 & 7 & 3 & 12 & 1 & & & 7 Flake scars \\
\hline 106 & 2 & $10-20$ & 1 & 2 & 1 & 0 & 1 & & & \\
\hline 106 & 2 & $10-20$ & 1 & 1 & 1 & 0 & 1 & & & \\
\hline 106 & 2 & $10-20$ & 1 & 2 & 1 & 38 & 2 & & & \\
\hline 106 & 2 & $20-30$ & 1 & 1 & 1 & 0 & 1 & & & \\
\hline 106 & 2 & $40-50$ & 1 & 4 & 1 & 0 & 1 & & & \\
\hline 106 & 2 & $40-50$ & 1 & 2 & 1 & 0 & 2 & & & \\
\hline 106 & 3 & $0-10$ & 1 & 2 & 1 & 0 & 3 & & & \\
\hline 106 & 3 & $0-10$ & 1 & 2 & 1 & 0 & 1 & & & \\
\hline 106 & 3 & $0-10$ & 1 & 2 & 1 & 0 & 1 & & & \\
\hline 106 & 3 & $0-10$ & - & - & - & - & - & & & 3 Burned bone fragments \\
\hline 106 & 4 & $0-10$ & 1 & 2 & 1 & 0 & 1 & & & Overshot flake \\
\hline 106 & 4 & $10-20$ & 1 & 2 & 1 & 12 & 1 & & & \\
\hline 106 & 4 & $10-20$ & 1 & 2 & 1 & 12 & 1 & & & \\
\hline 106 & 4 & $20-30$ & 1 & 3 & 1 & 0 & 1 & & & \\
\hline 106 & 4 & $20-30$ & 1 & 1 & 1 & 12 & 1 & & & \\
\hline 106 & 4 & $20-30$ & 1 & 2 & 1 & 12 & 1 & & & \\
\hline 106 & 4 & $30-40$ & 1 & 3 & 1 & 0 & 1 & & & \\
\hline 106 & 4 & $30-40$ & 1 & 2 & 1 & 0 & 1 & & & \\
\hline 106 & 4 & $30-40$ & 1 & 3 & 1 & 0 & 1 & & & \\
\hline 106 & 4 & $40-50$ & 1 & 2 & 1 & 0 & 1 & & & \\
\hline 106 & 4 & $40-50$ & 1 & 1 & 1 & 12 & 4 & & & \\
\hline 106 & 5 & $0-10$ & 1 & 3 & 1 & 12 & 1 & & & \\
\hline 106 & 5 & $0-10$ & 6 & 3 & 1 & 0 & 1 & & & \\
\hline 106 & 5 & $0-10$ & 1 & 2 & 1 & 0 & 1 & & & \\
\hline 106 & 5 & $0-10$ & 1 & 2 & 1 & 0 & 1 & & & \\
\hline 106 & 5 & $0-10$ & 1 & 2 & 1 & 0 & 1 & & & \\
\hline 106 & 5 & $0-10$ & 1 & 2 & 1 & 38 & 1 & & & \\
\hline 106 & 5 & $0-10$ & 1 & 1 & 1 & 0 & 2 & & & \\
\hline 106 & 5 & $10-20$ & 1 & 2 & 1 & 0 & 1 & Limestone & & \\
\hline
\end{tabular}


Table B-1. continued...

\begin{tabular}{|c|c|c|c|c|c|c|c|c|c|c|}
\hline $\begin{array}{r}\text { Site } \\
\text { 41TG }\end{array}$ & $\begin{array}{r}\text { Shovel } \\
\text { Test \# }\end{array}$ & $\begin{array}{c}\text { Depth } \\
\text { cm }\end{array}$ & $\begin{array}{c}\text { Artifact } \\
\text { Type }\end{array}$ & $\begin{array}{l}\text { Max. } \\
\text { Length }\end{array}$ & $\begin{array}{c}\text { Max. } \\
\text { Thickness }\end{array}$ & $\begin{array}{c}\text { Cortex } \\
\%\end{array}$ & \begin{tabular}{|c|} 
Material \\
Color
\end{tabular} & $\begin{array}{c}\text { Fire Cracked } \\
\text { Rock }\end{array}$ & $\begin{array}{c}\text { Mussel } \\
\text { Shell }\end{array}$ & $\begin{array}{c}\text { Other / } \\
\text { Remarks }\end{array}$ \\
\hline 106 & 5 & $10-20$ & 1 & 2 & 1 & 0 & 1 & & & \\
\hline 106 & 5 & $20-30$ & 1 & 1 & 1 & 0 & 1 & & & \\
\hline 106 & 5 & $20-30$ & 1 & 2 & 1 & 0 & 1 & & & \\
\hline 106 & 5 & $20-30$ & 1 & 2 & 1 & 0 & 1 & & & \\
\hline 106 & 5 & $20-30$ & 1 & 2 & 1 & 0 & 1 & & & \\
\hline 106 & 5 & $40-50$ & 1 & 3 & 2 & 12 & 2 & & & \\
\hline 106 & 5 & $40-50$ & 1 & 2 & 1 & 0 & 1 & & & \\
\hline 106 & 5 & $40-50$ & 1 & 2 & 1 & 12 & 1 & & & \\
\hline 106 & 5 & $40-50$ & 1 & 1 & 1 & 0 & 2 & & & \\
\hline 106 & 5 & $40-50$ & 1 & 3 & 1 & 38 & 1 & & & \\
\hline 106 & 5 & $40-50$ & 1 & 2 & 1 & 0 & 2 & & & \\
\hline 106 & 5 & $50-60$ & 1 & 2 & 1 & 0 & 2 & & & \\
\hline 109 & 3 & $0-10$ & - & - & - & - & - & Chert & & \\
\hline 109 & 3 & $0-10$ & 1 & 3 & 1 & 12 & 1 & & & \\
\hline 109 & 3 & $0-10$ & 1 & 2 & 1 & 0 & 1 & & & \\
\hline 109 & 5 & $10-20$ & 1 & 2 & 1 & 0 & 1 & & & \\
\hline 109 & 5 & $10-20$ & 1 & 2 & 1 & 0 & 1 & & & \\
\hline 109 & 8 & $10-20$ & 1 & 2 & 1 & 38 & 1 & & & \\
\hline 109 & 9 & $10-20$ & 6 & 1 & 2 & 0 & 1 & & & \\
\hline 110 & 1 & $0-10$ & 1 & 2 & 1 & 0 & 1 & Limestone & & Clear glass bottle sherd \\
\hline 117 & 1 & $0-10$ & 1 & 3 & 1 & 62 & 1 & & & \\
\hline 117 & 3 & $0-10$ & 1 & 3 & 1 & 0 & 1 & & & \\
\hline 117 & 5 & $0-10$ & 1 & 3 & 1 & 88 & 1 & & & \\
\hline 159 & 3 & $30-40$ & 1 & 2 & 1 & 88 & 1 & & & \\
\hline 159 & 4 & $0-10$ & 1 & 3 & 1 & 0 & 1 & & & \\
\hline 244 & 1 & $0-10$ & 1 & 3 & 1 & 88 & 1 & & & \\
\hline 244 & 1 & $0-10$ & 1 & 2 & 1 & 12 & 1 & & & \\
\hline 244 & 1 & $0-10$ & 1 & 2 & 1 & 100 & 1 & & & \\
\hline 244 & 1 & $0-10$ & 1 & 2 & 1 & 0 & 1 & & & \\
\hline 244 & 1 & $0-10$ & - & - & - & - & $\ldots$ & Chert & & \\
\hline 244 & 1 & $0-10$ & 1 & 4 & 1 & 62 & 1 & & & \\
\hline 244 & 1 & $0-10$ & 1 & 4 & 3 & 62 & 1 & & & \\
\hline 244 & 1 & $0-10$ & 1 & 3 & 1 & 12 & 1 & & & \\
\hline 244 & 1 & $10-20$ & 1 & 2 & 1 & 0 & 1 & & & \\
\hline 244 & 1 & $10-20$ & 1 & 1 & 1 & 0 & 1 & & & \\
\hline 244 & 1 & 10-20 & 1 & 2 & 1 & 88 & 1 & & & \\
\hline 244 & 1 & $10-20$ & - & - & - & - & - & & & Chert \\
\hline 244 & 2 & $0-10$ & 1 & 4 & 1 & 0 & 1 & & & Blade \\
\hline 244 & 2 & $0-10$ & 1 & 2 & 1 & 88 & 1 & & & \\
\hline 244 & 2 & $0-10$ & 6 & 5 & 1 & 12 & 4 & & & \\
\hline 244 & 2 & $0-10$ & 1 & 1 & 1 & 0 & 1 & & & \\
\hline 244 & 2 & $0-10$ & 1 & 2 & 1 & 12 & 1 & & & \\
\hline 244 & 5 & $0-7$ & 1 & 4 & 1 & 0 & 1 & & & \\
\hline 244 & 7 & $10-20$ & 1 & 1 & 1 & 0 & 1 & & & \\
\hline 244 & 7 & $10-20$ & - & - & - & - & - & & & Chert potlid \\
\hline 244 & 10 & $20-30$ & 1 & 2 & 1 & 12 & 4 & & & \\
\hline 244 & 13 & $0-10$ & 1 & 4 & 1 & 38 & 1 & & & \\
\hline
\end{tabular}


Table B-1. continued...

\begin{tabular}{|c|c|c|c|c|c|c|c|c|c|c|}
\hline $\begin{array}{r}\text { Site } \\
\text { 41TG }\end{array}$ & $\begin{array}{r}\text { Shovel } \\
\text { Test \# }\end{array}$ & $\begin{array}{c}\text { Depth } \\
\text { cm }\end{array}$ & $\begin{array}{c}\text { Artifact } \\
\text { Tvpe }\end{array}$ & $\begin{array}{c}\text { Max. } \\
\text { Length }\end{array}$ & $\begin{array}{c}\text { Max. } \\
\text { Thickness } \\
\end{array}$ & $\begin{array}{c}\text { Cortex } \\
\% \\
\end{array}$ & $\begin{array}{c}\text { Material } \\
\text { Color }\end{array}$ & $\begin{array}{c}\text { Fire Cracked } \\
\text { Rock }\end{array}$ & $\begin{array}{c}\text { Mussel } \\
\text { Shell }\end{array}$ & $\begin{array}{c}\text { Other / } \\
\text { Remarks }\end{array}$ \\
\hline 244 & 13 & $0-10$ & 1 & 3 & 1 & 12 & 1 & & & \\
\hline 244 & 13 & $0-10$ & 1 & 3 & 2 & 38 & 1 & & & \\
\hline 244 & 13 & $0-10$ & 1 & 2 & 1 & 0 & 1 & & & \\
\hline 244 & 13 & $0-10$ & 1 & 3 & 1 & 12 & 1 & & & \\
\hline 244 & 13 & $0-10$ & 1 & 1 & 1 & 0 & 1 & & & \\
\hline 244 & 13 & $0-10$ & 1 & 2 & 1 & 0 & 1 & & & \\
\hline 246 & 1 & $0-10$ & 1 & 5 & 1 & 0 & 1 & & & \\
\hline 246 & 2 & $10-20$ & 1 & 4 & 1 & 88 & 1 & & & \\
\hline 247 & 1 & $0-10$ & 1 & 3 & 1 & 0 & 1 & & & \\
\hline 247 & 1 & $0-10$ & 2 & 4 & 2 & 38 & 1 & & & \\
\hline 247 & 1 & $0-10$ & 1 & 1 & 1 & 0 & 1 & & & \\
\hline 247 & 1 & $0-10$ & 1 & 2 & 1 & 0 & 1 & & & \\
\hline 247 & 2 & $10-20$ & 6 & 4 & 2 & 0 & 1 & & & \\
\hline 247 & 2 & $10-20$ & 1 & 2 & 1 & 88 & 1 & & & \\
\hline 247 & 3 & $0-10$ & 1 & 3 & 1 & 0 & 1 & & & \\
\hline 247 & 5 & $0-10$ & 1 & 2 & 1 & 0 & 1 & & & \\
\hline 247 & 5 & $0-10$ & 1 & 2 & 1 & 0 & 1 & & & \\
\hline 247 & 5 & $0-10$ & 1 & 2 & 1 & 0 & 1 & & & \\
\hline 247 & 6 & $0-10$ & 1 & 5 & 1 & 12 & 1 & & & \\
\hline 247 & 6 & $0-10$ & 1 & 3 & 1 & 0 & 1 & & & \\
\hline 247 & 6 & $0-10$ & 1 & 2 & 1 & 0 & 1 & & & \\
\hline 247 & 8 & $0-10$ & 1 & 2 & 1 & 0 & 1 & & & \\
\hline 247 & 8 & $0-10$ & 1 & 2 & 1 & 0 & 2 & & & \\
\hline 247 & 8 & $0-10$ & 1 & 2 & 1 & 12 & 1 & & & \\
\hline 247 & 8 & $0-10$ & 1 & 2 & 1 & 0 & 1 & & & \\
\hline 247 & 8 & $0-10$ & 1 & 3 & 1 & 0 & 1 & & & \\
\hline 247 & 8 & $0-10$ & 1 & 2 & 1 & 0 & 1 & & & \\
\hline 247 & 9 & $0-10$ & 1 & 4 & 1 & 0 & 1 & & & \\
\hline 247 & 9 & $0-10$ & 1 & 2 & 1 & 0 & 1 & & & \\
\hline 247 & 9 & $0-10$ & 1 & 3 & 1 & 12 & 1 & & & \\
\hline 247 & 9 & $0-10$ & 1 & 3 & 1 & 0 & 1 & & & \\
\hline 247 & 9 & $0-10$ & 1 & 2 & 1 & 0 & 1 & & & \\
\hline 250 & 3 & $0-10$ & 1 & 3 & 1 & 62 & 1 & & & \\
\hline 250 & 5 & $0-10$ & 1 & 6 & 2 & 12 & 1 & & & \\
\hline 250 & 5 & $0-10$ & 6 & 3 & 1 & 0 & 1 & & & \\
\hline 252 & 2 & $10-20$ & 1 & 2 & 1 & 0 & 1 & & & \\
\hline 252 & 10 & $10-20$ & 1 & 3 & 1 & 0 & 1 & & & \\
\hline 252 & 10 & $10-20$ & 1 & 3 & 1 & 88 & 1 & & & \\
\hline 252 & 11 & $10-20$ & - & - & - & - & - & Chert & & \\
\hline 253 & 1 & $0-10$ & 1 & 3 & 1 & 12 & 1 & & & \\
\hline 253 & 1 & $40-50$ & 6 & 4 & 1 & 12 & 1 & & & \\
\hline 253 & 2 & $20-30$ & - & - & - & - & - & Sandstone & & \\
\hline 253 & 3 & $0-10$ & 1 & 2 & 1 & 0 & 1 & & & \\
\hline 253 & 3 & $0-10$ & 1 & 2 & 1 & 0 & 1 & & & \\
\hline 253 & 3 & $0-10$ & - & - & - & - & - & Limestone & & \\
\hline 253 & 3 & $10-20$ & 1 & 1 & 1 & 0 & 1 & & & \\
\hline 253 & 3 & $20-30$ & 1 & 2 & 1 & 0 & 1 & & & \\
\hline
\end{tabular}


Table B-1. continued...

\begin{tabular}{|c|c|c|c|c|c|c|c|c|c|c|}
\hline $\begin{array}{r}\text { Site } \\
\text { 41TG }\end{array}$ & $\begin{array}{l}\text { Shovel } \\
\text { Test \# }\end{array}$ & $\begin{array}{c}\text { Depth } \\
\text { cm }\end{array}$ & $\begin{array}{c}\text { Artifact } \\
\text { Type }\end{array}$ & $\begin{array}{l}\text { Max. } \\
\text { Length }\end{array}$ & $\begin{array}{c}\text { Max. } \\
\text { Thickness } \\
\end{array}$ & $\begin{array}{c}\text { Cortex } \\
\% \\
\end{array}$ & $\begin{array}{c}\text { Material } \\
\text { Color }\end{array}$ & $\begin{array}{c}\text { Fire Cracked } \\
\text { Rock }\end{array}$ & $\begin{array}{c}\text { Mussel } \\
\text { Shell }\end{array}$ & $\begin{array}{r}\text { Other / } \\
\text { Remarks }\end{array}$ \\
\hline 253 & 3 & $20-30$ & 1 & 5 & 1 & 100 & 1 & & & \\
\hline 253 & 3 & $30-40$ & 4 & 3 & 1 & 0 & 4 & & & \\
\hline 253 & 3 & $30-40$ & 1 & 1 & 1 & 0 & 1 & & & \\
\hline 253 & 3 & $30-40$ & 1 & 2 & 1 & 12 & 1 & & & \\
\hline 253 & 3 & $30-40$ & 1 & 2 & 1 & 12 & 4 & & & \\
\hline 253 & 3 & $30-40$ & 1 & 1 & 1 & 100 & 1 & & & \\
\hline 253 & 3 & $30-40$ & 1 & 2 & 1 & 0 & 1 & & & \\
\hline 253 & 4 & 45 & 6 & 3 & 1 & 0 & 3 & & & \\
\hline 253 & 4 & $0-10$ & 1 & 3 & 2 & 100 & 1 & & & \\
\hline 253 & 4 & $0-10$ & 1 & 2 & 1 & 0 & 1 & & & \\
\hline 253 & 4 & $10-20$ & 1 & 2 & 1 & 12 & 1 & & & \\
\hline 253 & 4 & $10-20$ & 1 & 3 & 1 & 100 & 1 & & & \\
\hline 253 & 4 & $10-20$ & 1 & 3 & 2 & 100 & 1 & & & \\
\hline 253 & 4 & $10-20$ & 1 & 1 & 1 & 0 & 1 & & & \\
\hline 253 & 4 & $10-20$ & 1 & 2 & 1 & 12 & 3 & & & \\
\hline 253 & 5 & $30-40$ & 1 & 5 & 1 & 0 & 1 & & & \\
\hline 253 & 5 & $30-40$ & 1 & 3 & 1 & 0 & 1 & & & \\
\hline 253 & 5 & $30-40$ & 1 & 2 & 1 & 12 & 1 & & & \\
\hline 253 & 5 & $30-40$ & 1 & 2 & 1 & 0 & 1 & & & \\
\hline 253 & 6 & $0-10$ & 6 & 4 & 1 & 12 & 1 & & & \\
\hline 253 & 6 & $0-10$ & 1 & 4 & 1 & 12 & 1 & & & \\
\hline 253 & 6 & $10-20$ & 1 & 4 & 1 & 12 & 1 & & & \\
\hline 253 & 6 & $20-30$ & 1 & 3 & 2 & 0 & 1 & & & \\
\hline 253 & 7 & $0-10$ & 1 & 2 & 1 & 0 & 1 & & & \\
\hline 253 & 7 & $0-10$ & 1 & 3 & 1 & 0 & 1 & & & \\
\hline 253 & 7 & $10-20$ & 1 & 2 & 1 & 0 & 1 & & & \\
\hline 253 & 7 & $10-20$ & 6 & 6 & 2 & 12 & 1 & & & \\
\hline 253 & 7 & $20-30$ & . & - & - & - & - & Limestone & & \\
\hline 253 & 8 & $0-10$ & 1 & 4 & 1 & 0 & 1 & & & \\
\hline 253 & 8 & $0-10$ & 1 & 5 & 2 & 88 & 1 & & & \\
\hline 253 & 8 & $10-20$ & 1 & 4 & 1 & 0 & 1 & & & \\
\hline 253 & 8 & $20-30$ & 1 & 2 & 1 & 0 & 1 & & & \\
\hline 253 & 8 & $20-30$ & 1 & 2 & 1 & 0 & 1 & & & \\
\hline 253 & 8 & $20-30$ & 1 & 2 & 1 & 0 & 1 & & & \\
\hline 253 & 8 & $20-30$ & 1 & 2 & 1 & 0 & 1 & & & \\
\hline 253 & 8 & $20-30$ & 1 & 3 & 1 & 0 & 1 & & & \\
\hline 253 & 9 & $0-10$ & 1 & 4 & 1 & 0 & 1 & & & \\
\hline 253 & 10 & $0-10$ & 6 & 3 & 1 & 0 & 1 & & & \\
\hline 253 & 10 & $0-10$ & - & - & - & - & - & Limestone & & \\
\hline 253 & 10 & $10-20$ & - & - & - & - & - & Limestone & & \\
\hline 253 & 10 & $20-30$ & 1 & 2 & 1 & 0 & 1 & & & \\
\hline 253 & 10 & $20-30$ & 1 & 1 & 1 & 12 & 3 & & & \\
\hline 362 & 4 & $0-10$ & 1 & 4 & 1 & 38 & 4 & & & \\
\hline 362 & 4 & $0-10$ & 1 & 2 & 1 & 12 & 1 & & & \\
\hline 364 & 3 & $0-10$ & 1 & 2 & 1 & 100 & 1 & & & \\
\hline 364 & 3 & $0-10$ & 1 & 3 & 1 & 0 & 1 & & & \\
\hline 364 & 3 & $0-10$ & 1 & 1 & 1 & 0 & 1 & & & \\
\hline
\end{tabular}


Table B-1. continued...

\begin{tabular}{|c|c|c|c|c|c|c|c|c|c|c|}
\hline $\begin{array}{r}\text { Site } \\
\text { 41TG } \\
\end{array}$ & $\begin{array}{l}\text { Shovel } \\
\text { Test \# }\end{array}$ & $\begin{array}{c}\text { Depth } \\
\text { cm }\end{array}$ & $\begin{array}{c}\text { Artifact } \\
\text { Type }\end{array}$ & $\begin{array}{c}\text { Max. } \\
\text { Length }\end{array}$ & $\begin{array}{c}\text { Max. } \\
\text { Thickness }\end{array}$ & $\begin{array}{c}\text { Cortex } \\
\% \\
\end{array}$ & \begin{tabular}{|c|}
$\begin{array}{c}\text { Material } \\
\text { Color }\end{array}$ \\
\end{tabular} & $\begin{array}{c}\begin{array}{c}\text { Fire Cracked } \\
\text { Rock }\end{array} \\
\end{array}$ & $\begin{array}{c}\text { Mussel } \\
\text { Shell }\end{array}$ & $\begin{array}{c}\text { Other / } \\
\text { Remarks } \\
\end{array}$ \\
\hline 364 & 5 & $0-10$ & 1 & 1 & 1 & 12 & 1 & & & \\
\hline 365 & 2 & $0-10$ & 1 & 2 & 1 & 0 & 1 & & & \\
\hline 366 & 4 & $0-10$ & 1 & 3 & 1 & 88 & 4 & & & \\
\hline 367 & 1 & $30-40$ & 1 & 1 & 1 & 12 & 1 & & & \\
\hline 367 & 1 & $30-40$ & 1 & 2 & 1 & 0 & 1 & & & \\
\hline 367 & 2 & $30-40$ & 1 & 4 & 2 & 0 & 1 & & & \\
\hline 367 & 4 & $30-40$ & 1 & 3 & 1 & 38 & 4 & & & \\
\hline 367 & 4 & $30-40$ & 1 & 2 & 1 & 12 & 3 & & & \\
\hline 367 & 4 & $30-40$ & - & - & - & - & - & Limestone & & \\
\hline 367 & 4 & $30-40$ & - & - & - & 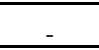 & - & Sandstone & & \\
\hline 372 & 1 & $20-30$ & 7 & - & - & _- & - & & & Yellow ochre \\
\hline 372 & 1 & $30-40$ & - & - & - & - & - & Limestone & & \\
\hline 372 & 2 & $0-10$ & 1 & 1 & 1 & 0 & 1 & & & \\
\hline 372 & 2 & $10-20$ & - & - & - & - & - & & $\mathrm{x}$ & \\
\hline 372 & 5 & $40-50$ & 1 & 2 & 1 & 100 & 4 & & & \\
\hline 372 & 9 & $0-10$ & 1 & 2 & 1 & 0 & 1 & & & \\
\hline 372 & 9 & $0-10$ & 1 & 2 & 1 & 0 & 1 & & & \\
\hline 372 & 9 & $0-10$ & 1 & 1 & 1 & 0 & 1 & & & \\
\hline 373 & 3 & $20-25$ & 2 & 5 & 1 & 0 & 1 & & & Retouched medial frag \\
\hline 373 & 3 & $20-25$ & 1 & 6 & 1 & 0 & 1 & & & Distal fragment \\
\hline 373 & 3 & $20-25$ & 1 & 2 & 1 & 0 & 1 & & & Medial fragment \\
\hline 373 & 6 & $20-30$ & - & - & - & - & - & & & Charcoal \\
\hline 373 & 6 & $30-40$ & - & - & - & - & - & & & Charcoal \\
\hline 374 & 2 & $40-50$ & 1 & 1 & 1 & 0 & 1 & & & \\
\hline 377 & 1 & $10-20$ & - & - & - & - & - & Limestone & & \\
\hline 377 & 1 & $20-30$ & - & - & _. & - & - & & & Charcoal \\
\hline 377 & 1 & $20-30$ & 1 & 2 & 1 & 0 & 1 & & & \\
\hline 377 & 1 & $20-30$ & 1 & 2 & 1 & 12 & 1 & & & \\
\hline 377 & 1 & $20-30$ & 1 & 1 & 1 & 0 & 1 & & & \\
\hline 377 & 1 & $20-30$ & 1 & 1 & 1 & 0 & 3 & & & \\
\hline 377 & 1 & $30-40$ & - & - & - & - & - & Limestone & & \\
\hline 377 & 3 & $60-70$ & 1 & 1 & 1 & 0 & 1 & & & \\
\hline 377 & 8 & $0-10$ & 3 & 4 & 4 & 75 & 4 & & & 2 Flake scars \\
\hline 377 & 8 & $0-10$ & 1 & 1 & 1 & 0 & 1 & & & \\
\hline 378 & 1 & $0-10$ & 1 & 3 & 1 & 100 & 1 & & & \\
\hline 378 & 1 & $0-10$ & 1 & 2 & 1 & 12 & 1 & & & \\
\hline 378 & 1 & $0-10$ & 1 & 2 & 1 & 88 & 1 & & & \\
\hline 378 & 6 & $0-10$ & 6 & 5 & 2 & 38 & 1 & & & \\
\hline 378 & 6 & $0-10$ & 1 & 3 & 1 & 12 & 1 & & & \\
\hline 378 & 6 & $0-10$ & 1 & 3 & 1 & 12 & 1 & & & \\
\hline 378 & 6 & $0-10$ & 1 & 3 & 1 & 12 & 1 & & & \\
\hline 378 & 6 & $0-10$ & 1 & 2 & 1 & 12 & 1 & & & \\
\hline 378 & 6 & $0-10$ & 1 & 3 & 1 & 12 & 1 & & & \\
\hline 378 & 6 & $0-10$ & 1 & 2 & 1 & 0 & 1 & & & \\
\hline 378 & 6 & $0-10$ & 1 & 1 & 1 & 0 & 1 & & & Burned \\
\hline 378 & 6 & $0-10$ & 1 & 2 & 1 & 100 & 3 & & & \\
\hline 378 & 6 & $0-10$ & 1 & 1 & 1 & 12 & 3 & & & \\
\hline
\end{tabular}


Table B-1. continued...

\begin{tabular}{|c|c|c|c|c|c|c|c|c|c|c|}
\hline $\begin{array}{r}\text { Site } \\
41 \mathrm{TG} \\
\end{array}$ & $\begin{array}{l}\text { Shovel } \\
\text { Test \# }\end{array}$ & $\begin{array}{c}\text { Depth } \\
\text { cm }\end{array}$ & $\begin{array}{c}\text { Artifact } \\
\text { Type }\end{array}$ & $\begin{array}{l}\text { Max. } \\
\text { Length }\end{array}$ & $\begin{array}{l}\text { Max. } \\
\text { Thickness }\end{array}$ & $\begin{array}{c}\text { Cortex } \\
\% \\
\end{array}$ & $\begin{array}{c}\text { Material } \\
\text { Color } \\
\end{array}$ & $\begin{array}{c}\text { Fire Cracked } \\
\text { Rock }\end{array}$ & $\begin{array}{c}\text { Mussel } \\
\text { Shell }\end{array}$ & $\begin{array}{c}\text { Other / } \\
\text { Remarks }\end{array}$ \\
\hline 378 & 6 & $0-10$ & 1 & 1 & 1 & 0 & 1 & & & \\
\hline 378 & 6 & $0-10$ & 1 & 1 & 1 & 12 & 1 & & & \\
\hline 378 & 6 & $0-10$ & 1 & 1 & 1 & 0 & 1 & & & \\
\hline 378 & 6 & $0-10$ & 1 & 1 & 1 & 0 & 1 & & & \\
\hline 378 & 6 & $0-10$ & 1 & 1 & 1 & 0 & 1 & & & \\
\hline 378 & 6 & $0-10$ & 1 & 1 & 1 & 0 & 1 & & & \\
\hline 378 & 6 & $0-10$ & 1 & 2 & 1 & 0 & 1 & & & \\
\hline 378 & 6 & $0-10$ & 1 & 2 & 1 & 0 & 1 & & & \\
\hline 378 & 6 & $0-10$ & 1 & 2 & 1 & 0 & 1 & & & \\
\hline 378 & 6 & $0-10$ & 1 & 1 & 1 & 0 & 1 & & & \\
\hline 378 & 6 & $0-10$ & 1 & 1 & 1 & 0 & 1 & & & \\
\hline 378 & 6 & $0-10$ & 1 & 1 & 1 & 0 & 1 & & & \\
\hline 378 & 6 & $0-10$ & 1 & 1 & 1 & 0 & 1 & & & \\
\hline 378 & 8 & $0-10$ & - & - & - & - & - & Limestone & & \\
\hline 380 & 2 & $50-70$ & 7 & - & - & - & - & & & Burned clay sample \\
\hline 380 & 3 & $50-60$ & - & - & - & - & - & & & Charcoal \\
\hline 380 & 7 & $0-10$ & 1 & 3 & 1 & 0 & 1 & & & \\
\hline 380 & 7 & $10-20$ & - & - & - & - & - & & & Charcoal \\
\hline 380 & 7 & $10-20$ & - & - & - & - & - & Sandstone & & \\
\hline 380 & 8 & $0-10$ & 1 & 8 & 2 & 0 & 1 & & & \\
\hline 380 & 8 & $0-10$ & 1 & 2 & 1 & 0 & 1 & & & \\
\hline 380 & 8 & $40-50$ & - & - & - & - & - & & & Charcoal \\
\hline 381 & 6 & $0-10$ & - & - & - & - & - & Limestone & & \\
\hline 382 & 1 & $0-10$ & 1 & 1 & 1 & 0 & 1 & & & \\
\hline 382 & 1 & $0-10$ & 1 & 2 & 1 & 0 & 1 & & & \\
\hline 382 & 1 & $0-10$ & - & - & - & - & - & Limestone & & \\
\hline 382 & 1 & $20-30$ & - & - & - & - & - & & & 2 Bone fragments \\
\hline 382 & 5 & $0-10$ & - & - & - & - & - & Sandstone & & \\
\hline 382 & 5 & $20-30$ & 1 & 2 & 1 & 0 & 1 & & & \\
\hline 382 & 6 & $0-10$ & 1 & 2 & 1 & 0 & 1 & & & \\
\hline 382 & 6 & $40-50$ & 1 & 3 & 1 & 0 & 1 & & & \\
\hline 382 & 17 & $20-30$ & - & - & - & - & - & Limestone & & \\
\hline 382 & 18 & $20-30$ & 1 & 1 & 1 & 0 & 1 & & & \\
\hline 382 & 18 & $20-30$ & 1 & 1 & 1 & 0 & 1 & & & \\
\hline 382 & 18 & $20-30$ & 1 & 1 & 1 & 88 & 1 & & & \\
\hline 382 & 18 & $20-30$ & 1 & 3 & 1 & 88 & 1 & & & \\
\hline 382 & 18 & $20-30$ & - & - & - & - & - & Sandstone & & \\
\hline 382 & 18 & $20-30$ & - & - & - & - & - & Limestone & & \\
\hline 382 & 18 & $30-40$ & 7 & - & - & - & - & & $x$ & \\
\hline 382 & 19 & $30-40$ & 1 & 2 & 1 & 0 & 1 & & & \\
\hline 382 & 19 & $30-40$ & 1 & 1 & 1 & 0 & 1 & & & \\
\hline 382 & 19 & $30-40$ & - & - & - & - & - & Limestone & & \\
\hline 382 & 19 & $30-40$ & - & - & - & - & - & Sandstone & & \\
\hline 382 & 20 & $20-30$ & 1 & 2 & 1 & 0 & 1 & & & \\
\hline 382 & 20 & $30-40$ & - & - & - & - & - & Limestone & & \\
\hline 384 & 2 & $0-10$ & 1 & 2 & 1 & 0 & 1 & & & \\
\hline 384 & 2 & $10-20$ & - & 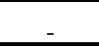 & 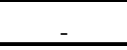 & - & 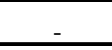 & Limestone & & \\
\hline
\end{tabular}


Table B-1. continued...

\begin{tabular}{|c|c|c|c|c|c|c|c|c|c|c|}
\hline $\begin{array}{r}\text { Site } \\
\text { 41TG } \\
\end{array}$ & $\begin{array}{l}\text { Shovel } \\
\text { Test \# }\end{array}$ & $\begin{array}{c}\text { Depth } \\
\text { cm }\end{array}$ & \begin{tabular}{|c|} 
Artifact \\
Type
\end{tabular} & $\begin{array}{l}\text { Max. } \\
\text { Length }\end{array}$ & $\begin{array}{c}\text { Max. } \\
\text { Thickness }\end{array}$ & $\begin{array}{c}\text { Cortex } \\
\%\end{array}$ & \begin{tabular}{|c|}
$\begin{array}{c}\text { Material } \\
\text { Color }\end{array}$ \\
\end{tabular} & $\begin{array}{c}\text { Fire Cracked } \\
\text { Rock }\end{array}$ & $\begin{array}{c}\begin{array}{c}\text { Mussel } \\
\text { Shell }\end{array} \\
\end{array}$ & $\begin{array}{c}\text { Other / } \\
\text { Remarks }\end{array}$ \\
\hline 384 & 3 & $10-20$ & - & - & - & - & - & Sandstone & & \\
\hline 384 & 3 & $10-20$ & - & - & - & - & - & & $\mathrm{X}$ & \\
\hline 384 & 3 & $20-30$ & - & - & - & - & - & & $\mathrm{X}$ & \\
\hline 384 & 4 & $0-10$ & 1 & 3 & 1 & 0 & 1 & & & \\
\hline 384 & 5 & $0-10$ & 1 & 4 & 1 & 0 & 1 & & & \\
\hline 384 & 5 & $0-10$ & 1 & 1 & 1 & 12 & 3 & & & \\
\hline 384 & 5 & $0-10$ & 1 & 1 & 1 & 0 & 1 & & & \\
\hline 384 & 5 & $0-10$ & - & - & - & - & - & Limestone & & \\
\hline 384 & 5 & $0-10$ & - & - & - & - & - & & $\mathrm{X}$ & \\
\hline 384 & 5 & $0-10$ & - & - & - & - & - & Limestone & & \\
\hline 384 & 5 & $10-20$ & 1 & 1 & 1 & 0 & 1 & & & \\
\hline 384 & 5 & $10-20$ & - & - & - & - & - & & $\mathrm{X}$ & \\
\hline 384 & 5 & $20-30$ & 7 & - & - & - & - & & & Pecan shell \\
\hline 384 & 6 & $0-10$ & 1 & 5 & 1 & 100 & 1 & & & \\
\hline 384 & 6 & $0-10$ & 1 & 2 & 1 & 0 & 1 & & & \\
\hline 384 & 6 & $20-30$ & - & - & - & - & - & Sandstone & & \\
\hline 384 & 6 & $20-30$ & 7 & - & - & - & - & & & Sandstone metate frag \\
\hline 384 & 6 & $30-40$ & - & - & - & - & - & Limestone & & \\
\hline 384 & 6 & $40-50$ & 1 & 6 & 2 & 88 & 1 & & & \\
\hline 384 & 6 & $40-50$ & 1 & 4 & 1 & 0 & 1 & & & \\
\hline 384 & 6 & $40-50$ & 1 & 4 & 1 & 0 & 1 & & & Refit \\
\hline 384 & 6 & $40-50$ & 1 & 3 & 1 & 0 & 1 & & & Refit \\
\hline 384 & 6 & $40-50$ & 1 & 2 & 1 & 100 & 1 & & & \\
\hline 384 & 6 & $40-50$ & - & - & - & - & - & Limestone & & \\
\hline 384 & 7 & $10-20$ & 1 & 1 & 1 & 0 & 1 & & & \\
\hline 384 & 7 & $10-20$ & 1 & 2 & 1 & 100 & 1 & & & \\
\hline 384 & 7 & $10-20$ & - & - & - & - & - & & $\mathrm{X}$ & \\
\hline 384 & 7 & $10-20$ & - & - & - & - & 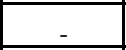 & Limestone & & \\
\hline 384 & 7 & $20-30$ & 1 & 1 & 1 & 88 & 1 & & & Burned \\
\hline 384 & 8 & $0-10$ & 1 & 4 & 1 & 88 & 3 & & & \\
\hline 384 & 8 & $10-20$ & - & - & - & - & - & Limestone & & \\
\hline 384 & 9 & $40-50$ & - & - & - & - & - & & $X$ & \\
\hline 384 & 9 & $40-50$ & - & - & - & - & 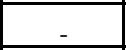 & & & Charcoal \\
\hline 384 & 10 & $0-10$ & 1 & 6 & 2 & 0 & 1 & & & \\
\hline 384 & 10 & $0-10$ & 1 & 2 & 1 & 12 & 1 & & & \\
\hline 384 & 10 & $0-10$ & 1 & 2 & 1 & 0 & 1 & & & Burned \\
\hline 384 & 10 & $0-10$ & 1 & 1 & 1 & 0 & 1 & & & Burned \\
\hline 384 & 10 & $0-10$ & 1 & 3 & 1 & 12 & 1 & & & Coarse grained \\
\hline 384 & 10 & $10-20$ & - & - & - & - & 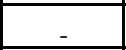 & & $\mathrm{X}$ & \\
\hline 384 & 11 & $0-10$ & - & - & - & - & - & & $\mathrm{X}$ & \\
\hline 384 & 11 & $0-10$ & 2 & 7 & 2 & 75 & 1 & & & 4 Flake scars \\
\hline 384 & 11 & $0-10$ & - & - & - & - & - & Limestone & & \\
\hline 384 & 11 & $0-10$ & 1 & 3 & 1 & 88 & 1 & & & \\
\hline 384 & 11 & $0-10$ & 1 & 4 & 1 & 12 & 1 & & & \\
\hline 384 & 11 & $10-20$ & 1 & 2 & 1 & 0 & 1 & & & \\
\hline 384 & 11 & $10-20$ & 1 & 2 & 1 & 0 & 1 & & & \\
\hline 384 & 11 & $20-30$ & - & - & - & - & - & Limestone & & \\
\hline
\end{tabular}


Table B-1. continued...

\begin{tabular}{|c|c|c|c|c|c|c|c|c|c|c|}
\hline $\begin{array}{r}\text { Site } \\
\text { 41TG } \\
\end{array}$ & $\begin{array}{l}\text { Shovel } \\
\text { Test \# }\end{array}$ & $\begin{array}{c}\text { Depth } \\
\text { cm }\end{array}$ & $\begin{array}{c}\text { Artifact } \\
\text { Type }\end{array}$ & $\begin{array}{c}\text { Max. } \\
\text { Length }\end{array}$ & $\begin{array}{c}\text { Max. } \\
\text { Thickness }\end{array}$ & $\begin{array}{c}\text { Cortex } \\
\% \\
\end{array}$ & $\begin{array}{c}\text { Material } \\
\text { Color } \\
\end{array}$ & $\begin{array}{c}\text { Fire Cracked } \\
\text { Rock } \\
\end{array}$ & $\begin{array}{c}\text { Mussel } \\
\text { Shell } \\
\end{array}$ & $\begin{array}{c}\text { Other / } \\
\text { Remarks }\end{array}$ \\
\hline 384 & 11 & $20-30$ & 1 & 2 & 1 & 0 & 1 & & & \\
\hline 384 & 11 & $20-30$ & 1 & 1 & 1 & 0 & 1 & & & \\
\hline 384 & 11 & $30-40$ & 1 & 4 & 1 & 12 & 1 & & & \\
\hline 384 & 11 & $30-40$ & 1 & 1 & 1 & 0 & 4 & & & \\
\hline 384 & 11 & $30-40$ & 1 & 4 & 1 & 88 & 1 & & & \\
\hline 384 & 12 & $0-10$ & 6 & 8 & 2 & 12 & 1 & & & \\
\hline 384 & 12 & $20-30$ & 1 & 2 & 1 & 0 & 1 & & & \\
\hline 384 & 12 & $20-30$ & - & - & - & - & - & & $\mathrm{X}$ & \\
\hline 384 & 12 & $20-30$ & - & - & - & - & - & Limestone & & \\
\hline 386 & 4 & $0-10$ & 6 & 3 & 1 & 0 & 1 & & & \\
\hline 386 & 4 & $0-10$ & 1 & 3 & 1 & 88 & 1 & & & \\
\hline 387 & 2 & $10-20$ & 1 & 3 & 2 & 12 & 1 & & & \\
\hline 388 & 4 & $30-40$ & - & - & - & - & - & & $\mathrm{X}$ & \\
\hline 388 & 4 & $30-40$ & - & - & - & - & - & & & Charcoal \\
\hline 388 & 4 & $30-40$ & - & - & - & - & - & & & Charcoal \\
\hline 388 & 6 & $0-10$ & - & - & - & - & - & Limestone & & \\
\hline 388 & 6 & $50-60$ & - & - & - & - & - & & $\mathrm{X}$ & \\
\hline 388 & 6 & $60-70$ & 1 & 1 & 1 & 0 & 1 & & & \\
\hline 388 & 6 & $60-70$ & - & - & - & - & - & Limestone & & \\
\hline 388 & 6 & $60-70$ & - & - & - & - & - & & $\mathrm{X}$ & \\
\hline 388 & 7 & $0-10$ & 1 & 7 & 1 & 38 & 1 & & & \\
\hline 388 & 7 & $0-10$ & 1 & 9 & 3 & 12 & 1 & & & \\
\hline 388 & 7 & $0-10$ & 2 & 10 & 3 & 25 & 1 & & 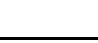 & 5 Flake scars \\
\hline 388 & 7 & $0-10$ & - & - & - & - & - & & $\mathrm{X}$ & \\
\hline 388 & 7 & $0-10$ & 1 & 2 & 1 & 38 & 1 & & & \\
\hline 388 & 7 & $0-10$ & - & - & - & - & - & Limestone & & \\
\hline 388 & 8 & $0-10$ & 1 & 4 & 1 & 0 & 1 & & & \\
\hline 388 & 8 & $0-10$ & 1 & 3 & 1 & 0 & 1 & & & \\
\hline 388 & 8 & $0-10$ & 1 & 3 & 1 & 0 & 1 & & & \\
\hline 388 & 8 & $0-10$ & 1 & 2 & 1 & 12 & 1 & & & \\
\hline 388 & 8 & $0-10$ & 1 & 2 & 1 & 0 & 1 & & & \\
\hline 388 & 8 & $0-10$ & - & - & - & - & - & Sandstone & & \\
\hline 388 & 8 & $10-20$ & - & - & - & - & - & Limestone & & \\
\hline 388 & 8 & $10-20$ & - & - & - & - & - & Sandstone & & \\
\hline 388 & 8 & $20-30$ & 1 & 2 & 1 & 0 & 1 & & & \\
\hline 388 & 8 & $20-30$ & 1 & 2 & 1 & 0 & 1 & & & \\
\hline 388 & 8 & $20-30$ & 1 & 3 & 1 & 0 & 1 & & & \\
\hline 388 & 8 & $20-30$ & 1 & 1 & 1 & 0 & 1 & & & \\
\hline 388 & 8 & $20-30$ & - & - & - & - & - & Limestone & & \\
\hline 388 & 8 & $30-40$ & - & - & - & - & - & Limestone & & \\
\hline 388 & 9 & $20-30$ & 1 & 1 & 1 & 0 & 1 & & & \\
\hline 388 & 9 & $30-40$ & 1 & 2 & 1 & 100 & 4 & & & \\
\hline 388 & 9 & $60-70$ & 1 & 2 & 1 & 0 & 1 & & & \\
\hline 388 & 11 & $0-10$ & - & - & - & - & - & & $\mathrm{X}$ & \\
\hline 388 & 11 & $10-20$ & 6 & 5 & 1 & 0 & 1 & & & \\
\hline 388 & 12 & $0-10$ & 1 & 2 & 1 & 0 & 1 & & & \\
\hline 388 & 12 & $0-10$ & 1 & 2 & 1 & 0 & 1 & & & \\
\hline
\end{tabular}


Table B-1. continued...

\begin{tabular}{|c|c|c|c|c|c|c|c|c|c|c|}
\hline $\begin{array}{r}\text { Site } \\
41 \mathrm{TG} \\
\end{array}$ & $\begin{array}{l}\text { Shovel } \\
\text { Test \# }\end{array}$ & $\begin{array}{c}\text { Depth } \\
\text { cm }\end{array}$ & \begin{tabular}{|c|}
$\begin{array}{c}\text { Artifact } \\
\text { Type }\end{array}$ \\
\end{tabular} & $\begin{array}{r}\text { Max. } \\
\text { Length }\end{array}$ & $\begin{array}{c}\text { Max. } \\
\text { Thickness }\end{array}$ & $\begin{array}{c}\text { Cortex } \\
\% \\
\end{array}$ & \begin{tabular}{|c|}
$\begin{array}{c}\text { Material } \\
\text { Color }\end{array}$ \\
\end{tabular} & $\begin{array}{c}\begin{array}{c}\text { Fire Cracked } \\
\text { Rock }\end{array} \\
\end{array}$ & $\begin{array}{c}\text { Mussel } \\
\text { Shell }\end{array}$ & $\begin{array}{c}\text { Other / } \\
\text { Remarks } \\
\end{array}$ \\
\hline 388 & 12 & $0-10$ & 1 & 2 & 1 & 12 & 1 & & & \\
\hline 388 & 12 & $0-10$ & 1 & 2 & 1 & 12 & 1 & & & \\
\hline 388 & 13 & $0-10$ & 1 & 2 & 1 & 0 & 1 & & & \\
\hline 388 & 13 & $30-40$ & 1 & 2 & 1 & 0 & 1 & & & \\
\hline 388 & 13 & $30-40$ & 1 & 2 & 1 & 0 & 1 & & & \\
\hline 388 & 14 & $10-20$ & 1 & 2 & 1 & 0 & 1 & & & \\
\hline 388 & 14 & $30-40$ & 1 & 3 & 1 & 38 & 2 & & & \\
\hline 388 & 14 & $50-60$ & 1 & 4 & 1 & 0 & 1 & & & \\
\hline 388 & AUGER & $100-120$ & 7 & - & - & - & - & & $\mathrm{X}$ & \\
\hline 388 & Auger & $60-80$ & - & - & - & - & - & & & Charcoal \\
\hline 389 & 1 & $0-10$ & 1 & 1 & 1 & 0 & 1 & & & \\
\hline 389 & 1 & $10-20$ & 1 & 2 & 1 & 0 & 1 & & & \\
\hline 389 & 1 & $10-20$ & 1 & 1 & 1 & 0 & 1 & & & \\
\hline 389 & 2 & $0-10$ & 1 & 2 & 1 & 88 & 1 & & & \\
\hline 389 & 2 & $0-10$ & 1 & 2 & 1 & 0 & 1 & & & \\
\hline 389 & 2 & $0-10$ & 1 & 2 & 1 & 0 & 3 & & & \\
\hline 389 & 2 & $0-10$ & 1 & 3 & 1 & 12 & 3 & & & \\
\hline 389 & 2 & $0-10$ & 1 & 2 & 1 & 12 & 1 & & & \\
\hline 389 & 2 & $0-10$ & 1 & 1 & 1 & 0 & 1 & & & \\
\hline 389 & 2 & $0-10$ & 1 & 1 & 1 & 0 & 1 & & & \\
\hline 389 & 2 & $0-10$ & 1 & 1 & 1 & 0 & 1 & & & \\
\hline 389 & 2 & $0-10$ & 1 & 2 & 1 & 0 & 1 & & & \\
\hline 389 & 2 & $0-10$ & 1 & 1 & 1 & 0 & 1 & & & \\
\hline 389 & 2 & $0-10$ & 1 & 1 & 1 & 0 & 9 & & & \\
\hline 389 & 2 & $0-10$ & - & - & - & - & - & Limestone & & \\
\hline 389 & 2 & $0-10$ & 7 & - & - & - & - & & & Clear glass sherd \\
\hline 389 & 2 & $10-20$ & - & - & - & - & - & Limestone & & \\
\hline 389 & 2 & $10-20$ & 1 & 3 & 1 & 0 & 9 & & & \\
\hline 389 & 2 & $10-20$ & 1 & 2 & 1 & 0 & 3 & & & \\
\hline 389 & 2 & $10-20$ & 1 & 3 & 1 & 12 & 1 & & & \\
\hline 389 & 2 & $10-20$ & 1 & 2 & 1 & 0 & 1 & & & \\
\hline 389 & 2 & $10-20$ & 1 & 2 & 1 & 0 & 1 & & & \\
\hline 389 & 2 & $10-20$ & - & - & - & - & - & Chert & & \\
\hline 389 & 2 & $10-20$ & 1 & 2 & 1 & 0 & 1 & & & \\
\hline 389 & 2 & $10-20$ & 1 & 2 & 1 & 0 & 1 & & & \\
\hline 389 & 2 & $10-20$ & 1 & 2 & 1 & 100 & 1 & & & \\
\hline 389 & 2 & $10-20$ & 1 & 2 & 1 & 12 & 12 & & & \\
\hline 389 & 2 & $10-20$ & 1 & 1 & 1 & 0 & 1 & & & \\
\hline 389 & 2 & $10-20$ & 1 & 2 & 1 & 12 & 1 & & & \\
\hline 389 & 2 & $10-20$ & 1 & 2 & 1 & 88 & 1 & & & \\
\hline 389 & 2 & $10-20$ & 1 & 1 & 1 & 12 & 1 & & & \\
\hline 389 & 2 & $20-30$ & 1 & 3 & 1 & 0 & 2 & Sandstone & & \\
\hline 389 & 2 & $20-30$ & 1 & 2 & 1 & 12 & 1 & & & \\
\hline 389 & 2 & $20-30$ & 1 & 2 & 1 & 0 & 1 & & & \\
\hline 389 & 2 & $30-40$ & 1 & 4 & 1 & 88 & 1 & & & \\
\hline 389 & 2 & $30-40$ & 1 & 2 & 1 & 12 & 1 & & & \\
\hline 389 & 2 & $30-40$ & 1 & 2 & 1 & 0 & 1 & & & \\
\hline
\end{tabular}


Table B-1. continued...

\begin{tabular}{|c|c|c|c|c|c|c|c|c|c|c|}
\hline $\begin{array}{r}\text { Site } \\
41 \mathrm{TG} \\
\end{array}$ & $\begin{array}{l}\text { Shovel } \\
\text { Test \# }\end{array}$ & $\begin{array}{c}\text { Depth } \\
\text { cm }\end{array}$ & $\begin{array}{c}\text { Artifact } \\
\text { Type }\end{array}$ & $\begin{array}{l}\text { Max. } \\
\text { Length }\end{array}$ & $\begin{array}{l}\text { Max. } \\
\text { Thickness }\end{array}$ & $\begin{array}{c}\text { Cortex } \\
\% \\
\end{array}$ & $\begin{array}{c}\text { Material } \\
\text { Color } \\
\end{array}$ & $\begin{array}{c}\text { Fire Cracked } \\
\text { Rock } \\
\end{array}$ & $\begin{array}{c}\text { Mussel } \\
\text { Shell } \\
\end{array}$ & $\begin{array}{r}\text { Other / } \\
\text { Remarks }\end{array}$ \\
\hline 389 & 2 & $30-40$ & 1 & 1 & 1 & 0 & 1 & & & \\
\hline 389 & 2 & $30-40$ & 1 & 1 & 1 & 0 & 1 & & & \\
\hline 389 & 2 & $40-50$ & 1 & 2 & 1 & 0 & 1 & & & \\
\hline 389 & 2 & $40-50$ & 1 & 2 & 1 & 0 & 1 & & & \\
\hline 389 & 3 & $0-10$ & 1 & 3 & 1 & 100 & 1 & & & \\
\hline 389 & 3 & $0-10$ & 1 & 5 & 1 & 12 & 1 & & & \\
\hline 389 & 3 & $0-10$ & 1 & 2 & 1 & 12 & 1 & & & \\
\hline 389 & 3 & $0-10$ & 1 & 4 & 1 & 0 & 1 & & & Blade \\
\hline 389 & 3 & $0-10$ & 1 & 1 & 1 & 0 & 1 & & & \\
\hline 389 & 3 & $0-10$ & 1 & 2 & 1 & 12 & 1 & & & \\
\hline 389 & 3 & $0-10$ & 1 & 2 & 1 & 88 & 1 & & & \\
\hline 389 & 3 & $0-10$ & 1 & 2 & 1 & 0 & 1 & & & \\
\hline 389 & 3 & $0-10$ & 1 & 2 & 1 & 0 & 1 & & & \\
\hline 389 & 3 & $0-10$ & 1 & 3 & 1 & 0 & 1 & & & \\
\hline 389 & 3 & $0-10$ & 1 & 2 & 1 & 0 & 1 & & & \\
\hline 389 & 3 & $0-10$ & 1 & 2 & 1 & 0 & 3 & & & \\
\hline 389 & 3 & $0-10$ & 1 & 2 & 1 & 12 & 1 & & & \\
\hline 389 & 3 & $0-10$ & 1 & 1 & 1 & 88 & 3 & & & \\
\hline 389 & 3 & $0-10$ & 1 & 2 & 1 & 0 & 1 & & & \\
\hline 389 & 3 & $0-10$ & 1 & 2 & 1 & 0 & 1 & & & \\
\hline 389 & 3 & $0-10$ & 1 & 1 & 1 & 0 & 1 & & & \\
\hline 389 & 3 & $0-10$ & 1 & 2 & 1 & 0 & 3 & & & \\
\hline 389 & 3 & $0-10$ & 1 & 2 & 1 & 12 & 1 & & & \\
\hline 389 & 3 & $0-10$ & 1 & 2 & 1 & 0 & 1 & & & \\
\hline 389 & 3 & $0-10$ & 1 & 2 & 1 & 0 & 1 & & & \\
\hline 389 & 3 & $0-10$ & 1 & 2 & 1 & 12 & 1 & & & \\
\hline 389 & 3 & $0-10$ & 1 & 2 & 1 & 0 & 1 & & & \\
\hline 389 & 3 & $0-10$ & 1 & 2 & 1 & 0 & 1 & & & \\
\hline 389 & 3 & $0-10$ & 1 & 2 & 1 & 88 & 1 & & & \\
\hline 389 & 3 & $0-10$ & 1 & 2 & 1 & 0 & 1 & & & \\
\hline 389 & 3 & $0-10$ & 1 & 1 & 1 & 0 & 1 & & & \\
\hline 389 & 3 & $0-10$ & - & - & - & - & - & Chert & & \\
\hline 389 & 3 & $10-20$ & 1 & 4 & 1 & 0 & 1 & & & \\
\hline 389 & 3 & $10-20$ & 1 & 4 & 1 & 88 & 1 & & & \\
\hline 389 & 3 & $10-20$ & 1 & 2 & 1 & 0 & 1 & & & \\
\hline 389 & 3 & $10-20$ & 1 & 3 & 1 & 100 & 3 & & & \\
\hline 389 & 3 & $10-20$ & 1 & 2 & 1 & 12 & 1 & & & \\
\hline 389 & 3 & $10-20$ & 1 & 2 & 1 & 0 & 1 & & & \\
\hline 389 & 3 & $10-20$ & 1 & 2 & 1 & 100 & 3 & & & \\
\hline 389 & 3 & $10-20$ & 1 & 1 & 1 & 12 & 1 & & & \\
\hline 389 & 3 & $20-30$ & 6 & 5 & 1 & 12 & 1 & & & \\
\hline 389 & 3 & $20-30$ & 1 & 2 & 1 & 38 & 1 & & & \\
\hline 389 & 3 & $20-30$ & 1 & 2 & 1 & 0 & 1 & & & \\
\hline 389 & 3 & 20-30 & 1 & 2 & 1 & 0 & 1 & & & \\
\hline 389 & 3 & $30-40$ & 1 & 1 & 1 & 0 & 1 & & & \\
\hline 389 & 4 & $0-10$ & 1 & 3 & 1 & 12 & 1 & & & \\
\hline 389 & 4 & $0-10$ & 1 & 3 & 1 & 0 & 1 & & & \\
\hline
\end{tabular}


Table B-1. continued...

\begin{tabular}{|c|c|c|c|c|c|c|c|c|c|c|}
\hline $\begin{array}{r}\text { Site } \\
41 \mathrm{TG} \\
\end{array}$ & $\begin{array}{l}\text { Shovel } \\
\text { Test \# }\end{array}$ & $\begin{array}{c}\text { Depth } \\
\text { cm }\end{array}$ & $\begin{array}{c}\text { Artifact } \\
\text { Type }\end{array}$ & $\begin{array}{c}\text { Max. } \\
\text { Length }\end{array}$ & $\begin{array}{c}\text { Max. } \\
\text { Thickness } \\
\end{array}$ & $\begin{array}{c}\text { Cortex } \\
\% \\
\end{array}$ & \begin{tabular}{|c|} 
Material \\
Color
\end{tabular} & $\begin{array}{c}\text { Fire Cracked } \\
\text { Rock }\end{array}$ & $\begin{array}{c}\text { Mussel } \\
\text { Shell }\end{array}$ & $\begin{array}{c}\text { Other / } \\
\text { Remarks }\end{array}$ \\
\hline 389 & 4 & $0-10$ & 1 & 3 & 1 & 0 & 1 & & & \\
\hline 389 & 4 & $0-10$ & - & - & - & - & - & Sandstone & & \\
\hline 389 & 4 & $10-20$ & 1 & 2 & 1 & 0 & 1 & & & \\
\hline 389 & 4 & $10-20$ & 1 & 2 & 1 & 12 & 1 & & & \\
\hline 389 & 4 & $10-20$ & 1 & 1 & 1 & 0 & 2 & & & \\
\hline 389 & 4 & $20-30$ & 1 & 3 & 1 & 0 & 1 & & & \\
\hline 389 & 4 & $20-30$ & 1 & 2 & 1 & 0 & 1 & & & \\
\hline 389 & 4 & $20-30$ & 1 & 2 & 1 & 0 & 1 & Sandstone & & \\
\hline 389 & 4 & $20-30$ & 1 & 2 & 1 & 0 & 3 & & & \\
\hline 389 & 4 & $20-30$ & 1 & 1 & 1 & 0 & 9 & & & Color is black \\
\hline 389 & 4 & $20-30$ & 1 & 1 & 1 & 0 & 1 & & & \\
\hline 389 & 5 & $0-10$ & 6 & 1 & 5 & 88 & 1 & & & \\
\hline 389 & 5 & $30-40$ & 1 & 2 & 1 & 0 & 1 & & & \\
\hline 389 & 6 & $0-10$ & 1 & 5 & 2 & 12 & 1 & & & \\
\hline 389 & 6 & $0-10$ & 1 & 1 & 1 & 12 & 1 & & & \\
\hline 389 & 6 & $0-10$ & 1 & 1 & 1 & 0 & 1 & & & \\
\hline 389 & 6 & $0-10$ & 1 & 1 & 1 & 0 & 1 & & & \\
\hline 389 & AUGER & $0-20$ & 1 & 2 & 1 & 100 & 1 & & & \\
\hline 389 & AUGER & $0-20$ & 1 & 2 & 1 & 1 & 1 & & & \\
\hline 389 & AUGER & $0-20$ & - & - & - & - & - & & $\mathrm{X}$ & \\
\hline 389 & AUGER & $0-20$ & - & - & - & - & - & & & Chert potlid \\
\hline 391 & 1 & $0-10$ & 1 & 3 & 1 & 0 & 1 & & & \\
\hline 391 & 1 & $0-10$ & 1 & 2 & 1 & 0 & 1 & & & \\
\hline 391 & 3 & $0-10$ & 1 & 1 & 1 & 0 & 1 & & & \\
\hline 391 & 5 & $10-20$ & 1 & 3 & 1 & 0 & 1 & & & \\
\hline 391 & 9 & $0-10$ & 1 & 3 & 1 & 100 & 1 & & & \\
\hline 391 & 9 & $10-20$ & 1 & 1 & 1 & 88 & 1 & & & \\
\hline 391 & 9 & $10-20$ & 1 & 2 & 1 & 88 & 1 & & & \\
\hline 391 & 9 & $10-20$ & 1 & 2 & 1 & 12 & 1 & & & \\
\hline 393 & 6 & $0-10$ & 1 & 3 & 1 & 0 & 1 & & & \\
\hline 394 & 1 & 73 & 7 & - & - & - & - & & & Ochre \\
\hline 394 & 1 & $60-70$ & - & - & - & - & - & Limestone & & \\
\hline 395 & 2 & $0-10$ & 1 & 3 & 1 & 0 & 1 & & & \\
\hline 396 & 3 & $0-10$ & 1 & 3 & 1 & 0 & 1 & & & \\
\hline 399 & 3 & $0-10$ & 1 & 3 & 1 & 88 & 1 & & & \\
\hline 399 & 3 & $0-10$ & 1 & 4 & 1 & 62 & 1 & & & \\
\hline 399 & 3 & $0-10$ & 1 & 2 & 1 & 0 & 1 & & & \\
\hline 399 & 3 & $0-10$ & 1 & 2 & 1 & 88 & 1 & & & \\
\hline 399 & 3 & $0-10$ & - & - & - & - & - & Chert & & \\
\hline 399 & 4 & $0-10$ & 1 & 3 & 1 & 100 & 1 & & & \\
\hline 399 & 4 & $10-20$ & 1 & 2 & 1 & 100 & 1 & & & \\
\hline 399 & 4 & $20-30$ & 1 & 2 & 1 & 0 & 1 & & & \\
\hline 399 & 5 & $0-10$ & 1 & 3 & 1 & 12 & 1 & & & \\
\hline 399 & 6 & $0-10$ & 1 & 3 & 1 & 0 & 1 & & & \\
\hline 399 & 7 & $20-30$ & 1 & 1 & 1 & 0 & 1 & & & \\
\hline 399 & 9 & $0-10$ & 1 & 5 & 2 & 12 & 1 & & & \\
\hline 399 & 9 & $0-10$ & 1 & 2 & 1 & 38 & 1 & & & \\
\hline
\end{tabular}


Table B-1. continued...

\begin{tabular}{|c|c|c|c|c|c|c|c|c|c|c|}
\hline $\begin{array}{r}\text { Site } \\
\text { 41TG } \\
\end{array}$ & $\begin{array}{l}\text { Shovel } \\
\text { Test \# }\end{array}$ & $\begin{array}{c}\text { Depth } \\
\text { cm }\end{array}$ & $\begin{array}{c}\text { Artifact } \\
\text { Type }\end{array}$ & $\begin{array}{c}\text { Max. } \\
\text { Length }\end{array}$ & $\begin{array}{c}\text { Max. } \\
\text { Thickness }\end{array}$ & $\begin{array}{c}\text { Cortex } \\
\% \\
\end{array}$ & $\begin{array}{c}\text { Material } \\
\text { Color }\end{array}$ & $\begin{array}{c}\begin{array}{c}\text { Fire Cracked } \\
\text { Rock }\end{array} \\
\end{array}$ & $\begin{array}{c}\text { Mussel } \\
\text { Shell }\end{array}$ & $\begin{array}{r}\text { Other / } \\
\text { Remarks }\end{array}$ \\
\hline 399 & 9 & $0-10$ & 1 & 1 & 1 & 10 & 1 & & & \\
\hline 399 & 9 & $0-10$ & - & - & - & - & - & Chert & & \\
\hline 399 & 9 & 10-20 & 1 & 3 & 1 & 88 & 1 & & & \\
\hline 400 & 1 & $0-10$ & 1 & 4 & 1 & 88 & 1 & & & \\
\hline 403 & 3 & $60-70$ & 1 & 5 & 1 & 88 & 1 & & & \\
\hline 404 & 3 & $0-10$ & 1 & 5 & 1 & 38 & 2 & & & \\
\hline 405 & 2 & $0-10$ & 1 & 2 & 1 & 0 & 1 & & & \\
\hline 405 & 3 & $20-30$ & 1 & 3 & 1 & 0 & 1 & & & \\
\hline 405 & 3 & $20-30$ & 1 & 1 & 1 & 0 & 1 & & & \\
\hline 405 & 3 & $30-40$ & 1 & 3 & 1 & 0 & 1 & & & \\
\hline 405 & 5 & $0-10$ & 1 & 2 & 1 & 12 & 1 & & & \\
\hline 405 & 6 & $20-30$ & 1 & 1 & 1 & 0 & 1 & & & \\
\hline 405 & 7 & $0-10$ & 1 & 2 & 1 & 0 & 1 & & & \\
\hline 405 & 7 & $0-10$ & 1 & 2 & 1 & 12 & 1 & & & \\
\hline 405 & 7 & $0-10$ & 1 & 2 & 1 & 0 & 1 & & & \\
\hline 405 & 7 & $0-10$ & 1 & 2 & 1 & 12 & 1 & & & \\
\hline 405 & 7 & $0-10$ & 1 & 3 & 1 & 0 & 1 & & & \\
\hline 405 & 7 & $0-10$ & 1 & 2 & 1 & 0 & 1 & & & \\
\hline 405 & 7 & $0-10$ & 1 & 2 & 1 & 0 & 1 & & & \\
\hline 405 & 7 & $0-10$ & 1 & 2 & 1 & 0 & 1 & & & \\
\hline 405 & 7 & $0-10$ & 1 & 1 & 1 & 0 & 1 & & & \\
\hline 405 & 8 & $30-40$ & 1 & 4 & 1 & 12 & 1 & & & \\
\hline 405 & 12 & $10-20$ & 1 & 3 & 1 & 0 & 4 & & & \\
\hline 406 & 1 & $30-40$ & 1 & 1 & 1 & 12 & 1 & & & \\
\hline 406 & 1 & $30-40$ & - & - & - & - & - & Limestone & & \\
\hline 406 & 2 & $0-10$ & 1 & 4 & 1 & 0 & 1 & & & \\
\hline 406 & 2 & $0-10$ & 1 & 2 & 1 & 0 & 1 & & & \\
\hline 406 & 2 & $0-10$ & 1 & 1 & 1 & 0 & 1 & & & \\
\hline 406 & 2 & $0-10$ & 1 & 2 & 1 & 0 & 1 & & & \\
\hline 406 & 2 & $0-10$ & 1 & 1 & 1 & 0 & 1 & & & \\
\hline 406 & 2 & $0-10$ & 1 & 2 & 1 & 0 & 1 & & & \\
\hline 406 & 2 & $0-10$ & 1 & 1 & 1 & 0 & 1 & & & \\
\hline 406 & 3 & $0-10$ & 1 & 3 & 1 & 0 & 1 & & & \\
\hline 406 & 3 & $0-10$ & 1 & 2 & 1 & 0 & 1 & & & \\
\hline 406 & 3 & $0-10$ & 1 & 1 & 1 & 0 & 1 & & & \\
\hline 406 & 3 & 20-30 & 1 & 5 & 1 & 88 & 1 & & & \\
\hline 406 & 3 & $20-30$ & 1 & 4 & 1 & 0 & 3 & & & \\
\hline 406 & 4 & $10-20$ & 1 & 2 & 1 & 0 & 3 & & & \\
\hline 408 & 3 & $0-10$ & 6 & 3 & 1 & 0 & 1 & & & \\
\hline 408 & 3 & $0-10$ & 1 & 3 & 1 & 62 & 1 & & & \\
\hline 408 & 3 & $0-10$ & 1 & 3 & 1 & 0 & 1 & & & \\
\hline 408 & 3 & $0-10$ & 1 & 2 & 1 & 38 & 1 & & & \\
\hline 408 & 3 & $0-10$ & 1 & 3 & 1 & 12 & 1 & & & \\
\hline 408 & 3 & $0-10$ & 6 & 3 & 1 & 0 & 1 & & & \\
\hline 409 & 1 & $0-10$ & 1 & 7 & 2 & 38 & 1 & & & \\
\hline 409 & 1 & $0-10$ & 5 & 3 & 1 & 0 & 1 & & & Fragment \\
\hline 409 & 1 & $0-10$ & 1 & 3 & 1 & 88 & 3 & & & \\
\hline
\end{tabular}


Table B-1. continued...

\begin{tabular}{|c|c|c|c|c|c|c|c|c|c|c|}
\hline $\begin{array}{r}\text { Site } \\
\text { 41TG } \\
\end{array}$ & $\begin{array}{r}\text { Shovel } \\
\text { Test \# }\end{array}$ & $\begin{array}{c}\text { Depth } \\
\text { cm }\end{array}$ & $\begin{array}{c}\text { Artifact } \\
\text { Type }\end{array}$ & $\begin{array}{c}\text { Max. } \\
\text { Length }\end{array}$ & $\begin{array}{c}\text { Max. } \\
\text { Thickness }\end{array}$ & $\begin{array}{c}\text { Cortex } \\
\% \\
\end{array}$ & $\begin{array}{c}\text { Material } \\
\text { Color }\end{array}$ & $\begin{array}{c}\text { Fire Cracked } \\
\text { Rock }\end{array}$ & $\begin{array}{c}\text { Mussel } \\
\text { Shell }\end{array}$ & $\begin{array}{r}\text { Other / } \\
\text { Remarks }\end{array}$ \\
\hline 409 & 1 & $0-10$ & 1 & 2 & 1 & 0 & 1 & & & \\
\hline 409 & 1 & $0-10$ & 1 & 2 & 1 & 0 & 1 & & & \\
\hline 409 & 1 & $0-10$ & 1 & 1 & 1 & 0 & 1 & & & \\
\hline 409 & 1 & $0-10$ & 1 & 2 & 1 & 12 & 1 & & & \\
\hline 409 & 1 & $0-10$ & 1 & 2 & 1 & 0 & 1 & & & \\
\hline 409 & 1 & $0-10$ & 1 & 2 & 1 & 0 & 1 & & & \\
\hline 409 & 1 & $0-10$ & 1 & 2 & 1 & 12 & 1 & & & \\
\hline 409 & 1 & $0-10$ & 1 & 2 & 1 & 12 & 1 & & & \\
\hline 409 & 1 & $0-10$ & 1 & 2 & 1 & 12 & 1 & & & \\
\hline 409 & 1 & $0-10$ & 1 & 2 & 1 & 0 & 3 & & & \\
\hline 409 & 1 & $0-10$ & 7 & - & - & - & _. & & & Ceramic \\
\hline 409 & 1 & $0-10$ & - & - & - & - & - & Limestone & & \\
\hline 409 & 1 & $0-10$ & - & - & - & - & - & Chert & & \\
\hline 409 & 1 & $10-20$ & 1 & 2 & 1 & 88 & 1 & & & \\
\hline 409 & 1 & $10-20$ & 1 & 4 & 1 & 62 & 1 & & & \\
\hline 409 & 1 & $10-20$ & 1 & 4 & 1 & 88 & 1 & & & \\
\hline 409 & 1 & $10-20$ & 1 & 5 & 1 & 0 & 1 & & & \\
\hline 409 & 1 & 10-20 & 1 & 3 & 1 & 38 & 3 & & & \\
\hline 409 & 1 & $10-20$ & 1 & 2 & 1 & 12 & 1 & & & \\
\hline 409 & 1 & $10-20$ & 1 & 3 & 1 & 0 & 1 & & & \\
\hline 409 & 1 & $10-20$ & 1 & 2 & 1 & 0 & 1 & & & \\
\hline 409 & 1 & $10-20$ & 1 & 1 & 1 & 0 & 1 & & & \\
\hline 409 & 1 & $10-20$ & 1 & 2 & 1 & 0 & 1 & & & \\
\hline 409 & 1 & $10-20$ & 1 & 2 & 1 & 88 & 1 & & & \\
\hline 409 & 1 & $10-20$ & 1 & 2 & 1 & 12 & 3 & & & \\
\hline 409 & 1 & $10-20$ & 1 & 2 & 1 & 0 & 1 & & & \\
\hline 409 & 1 & $10-20$ & 1 & 2 & 1 & 0 & 1 & & & \\
\hline 409 & 1 & $10-20$ & 1 & 2 & 1 & 0 & 1 & & & \\
\hline 409 & 1 & $10-20$ & 1 & 2 & 1 & 0 & 1 & & & \\
\hline 409 & 1 & $10-20$ & 1 & 2 & 1 & 0 & 1 & & & \\
\hline 409 & 1 & 10-20 & 1 & 2 & 1 & 0 & 1 & & & \\
\hline 409 & 1 & $10-20$ & 1 & 1 & 1 & 0 & 1 & & & \\
\hline 409 & 1 & $10-20$ & 1 & 2 & 1 & 88 & 3 & & & \\
\hline 409 & 1 & $10-20$ & 1 & 2 & 1 & 0 & 1 & & & \\
\hline 409 & 1 & $10-20$ & 1 & 1 & 1 & 0 & 1 & & & \\
\hline 409 & 1 & $10-20$ & - & - & - & - & - & Limestone & & \\
\hline 409 & 1 & $20-30$ & 2 & 5 & 3 & 62 & 1 & & & \\
\hline 409 & 1 & $20-30$ & 1 & 2 & 1 & 0 & 1 & & & \\
\hline 409 & 1 & $20-30$ & 1 & 2 & 1 & 0 & 1 & & & \\
\hline 409 & 1 & $20-30$ & 1 & 2 & 1 & 0 & 1 & & & \\
\hline 409 & 1 & $20-30$ & 1 & 2 & 1 & 0 & 1 & & & \\
\hline 409 & 1 & $20-30$ & 1 & 2 & 1 & 12 & 1 & & & \\
\hline 409 & 1 & $20-30$ & 1 & 2 & 1 & 12 & 3 & & & \\
\hline 409 & 1 & $20-30$ & 1 & 2 & 1 & 0 & 1 & & & \\
\hline 409 & 1 & $20-30$ & - & - & - & - & - & Limestone & & \\
\hline 409 & 1 & $30-40$ & 1 & 5 & 3 & 88 & 1 & & & \\
\hline 409 & 1 & $30-40$ & 1 & 4 & 1 & 0 & 1 & & & \\
\hline
\end{tabular}


Table B-1. continued...

\begin{tabular}{|c|c|c|c|c|c|c|c|c|c|c|}
\hline $\begin{array}{r}\text { Site } \\
41 \mathrm{TG} \\
\end{array}$ & $\begin{array}{l}\text { Shovel } \\
\text { Test \# }\end{array}$ & $\begin{array}{c}\text { Depth } \\
\text { cm }\end{array}$ & $\begin{array}{c}\text { Artifact } \\
\text { Tvpe }\end{array}$ & $\begin{array}{l}\text { Max. } \\
\text { Length }\end{array}$ & $\begin{array}{c}\text { Max. } \\
\text { Thickness }\end{array}$ & $\begin{array}{c}\text { Cortex } \\
\% \\
\end{array}$ & $\begin{array}{c}\text { Material } \\
\text { Color }\end{array}$ & $\begin{array}{c}\text { Fire Cracked } \\
\text { Rock } \\
\end{array}$ & $\begin{array}{c}\text { Mussel } \\
\text { Shell }\end{array}$ & $\begin{array}{c}\text { Other / } \\
\text { Remarks }\end{array}$ \\
\hline 409 & 1 & $30-40$ & 1 & 1 & 1 & 0 & 1 & & & \\
\hline 409 & 1 & $30-40$ & 1 & 2 & 1 & 0 & 1 & & & \\
\hline 409 & 1 & $30-40$ & 1 & 3 & 1 & 0 & 1 & & & \\
\hline 409 & 1 & $30-40$ & 1 & 3 & 1 & 0 & 1 & & & \\
\hline 409 & 1 & $30-40$ & 1 & 2 & 1 & 0 & 1 & & & \\
\hline 409 & 1 & $30-40$ & 1 & 3 & 1 & 0 & 1 & & & \\
\hline 409 & 1 & $30-40$ & 1 & 2 & 1 & 0 & 1 & & & \\
\hline 409 & 1 & $30-40$ & 7 & - & - & - & - & & & 3 Metate fragments \\
\hline 409 & 1 & $40-50$ & 1 & 3 & 1 & 0 & 1 & & & \\
\hline 409 & 1 & $40-50$ & 1 & 2 & 1 & 0 & 1 & & & \\
\hline 409 & 1 & $40-50$ & 1 & 2 & 1 & 0 & 1 & & & \\
\hline 409 & 1 & $40-50$ & 1 & 2 & 1 & 0 & 1 & & & \\
\hline 409 & 1 & $40-50$ & 1 & 2 & 1 & 88 & 1 & & & \\
\hline 409 & 1 & $40-50$ & - & - & - & - & - & Limestone & & \\
\hline 409 & 1 & $50-60$ & 1 & 7 & 1 & 38 & 1 & & & \\
\hline 409 & 1 & $50-60$ & 1 & 5 & 2 & 12 & 1 & & & \\
\hline 409 & 1 & $50-60$ & 1 & 3 & 1 & 0 & 1 & & & \\
\hline 409 & 1 & $50-60$ & 1 & 4 & 1 & 12 & 1 & & & \\
\hline 409 & 1 & $50-60$ & 1 & 4 & 1 & 12 & 1 & & & \\
\hline 409 & 1 & $50-60$ & 1 & 3 & 1 & 0 & 1 & & & \\
\hline 409 & 1 & $50-60$ & 1 & 2 & 1 & 0 & 1 & & & \\
\hline 409 & 1 & $50-60$ & 1 & 2 & 1 & 0 & 3 & & & \\
\hline 409 & 1 & $50-60$ & 1 & 2 & 1 & 0 & 1 & & & \\
\hline 409 & 1 & $50-60$ & 1 & 2 & 1 & 0 & 1 & & & \\
\hline 409 & 1 & $50-60$ & 1 & 2 & 1 & 0 & 1 & & & \\
\hline 409 & 1 & 50-60 & 1 & 2 & 1 & 0 & 1 & & & \\
\hline 409 & 1 & $50-60$ & 1 & 1 & 1 & 0 & 1 & & & \\
\hline 409 & 1 & $50-60$ & 1 & 1 & 1 & 0 & 1 & & & \\
\hline 409 & 1 & $50-60$ & 1 & 2 & 1 & 0 & 1 & & & \\
\hline 409 & 1 & $50-60$ & 1 & 2 & 1 & 0 & 1 & & & \\
\hline 409 & 1 & $50-60$ & 1 & 2 & 1 & 12 & 1 & & & \\
\hline 409 & 1 & $50-60$ & - & - & - & - & - & Sandstone & & \\
\hline 409 & 1 & $50-60$ & - & - & - & - & - & Limestone & & \\
\hline 409 & 1 & $50-60$ & - & - & - & - & - & Chert & & \\
\hline 409 & 1 & $60-70$ & 1 & 6 & 1 & 38 & 1 & & & \\
\hline 409 & 1 & $60-70$ & 1 & 6 & 1 & 88 & 1 & & & \\
\hline 409 & 1 & $60-70$ & 1 & 3 & 1 & 62 & 1 & & & \\
\hline 409 & 1 & $60-70$ & 1 & 2 & 1 & 0 & 1 & & & \\
\hline 409 & 1 & $60-70$ & 1 & 2 & 1 & 0 & 1 & & & \\
\hline 409 & 1 & $60-70$ & 1 & 3 & 1 & 0 & 1 & & & \\
\hline 409 & 1 & $60-70$ & 1 & 2 & 1 & 0 & 1 & & & \\
\hline 409 & 1 & $60-70$ & 1 & 2 & 1 & 0 & 1 & & & \\
\hline 409 & 1 & $60-70$ & 1 & 1 & 1 & 100 & 1 & & & \\
\hline 409 & 2 & $0-10$ & 1 & 3 & 1 & 12 & 1 & & & Bone \& clear glass \\
\hline 409 & 2 & $0-10$ & 1 & 2 & 1 & 0 & 1 & Chert \& Limestone & & \\
\hline 409 & 2 & $0-10$ & 1 & 3 & 1 & 0 & 1 & & & \\
\hline 409 & 2 & $0-10$ & 1 & 2 & 1 & 0 & 1 & & & \\
\hline
\end{tabular}


Table B-1. continued...

\begin{tabular}{|c|c|c|c|c|c|c|c|c|c|c|}
\hline $\begin{array}{r}\text { Site } \\
\text { 41TG } \\
\end{array}$ & $\begin{array}{l}\text { Shovel } \\
\text { Test \# }\end{array}$ & $\begin{array}{c}\text { Depth } \\
\mathrm{cm}\end{array}$ & \begin{tabular}{|c}
$\begin{array}{c}\text { Artifact } \\
\text { Tvpe }\end{array}$ \\
\end{tabular} & $\begin{array}{c}\text { Max. } \\
\text { Length }\end{array}$ & $\begin{array}{c}\text { Max. } \\
\text { Thickness }\end{array}$ & $\begin{array}{c}\text { Cortex } \\
\% \\
\end{array}$ & \begin{tabular}{|c|} 
Material \\
Color \\
\end{tabular} & $\begin{array}{c}\text { Fire Cracked } \\
\text { Rock }\end{array}$ & $\begin{array}{c}\text { Mussel } \\
\text { Shell } \\
\end{array}$ & $\begin{array}{c}\text { Other / } \\
\text { Remarks }\end{array}$ \\
\hline 409 & 2 & $0-10$ & 1 & 2 & 1 & 0 & 1 & & & \\
\hline 409 & 2 & $0-10$ & 1 & 2 & 1 & 0 & 1 & & & \\
\hline 409 & 2 & $0-10$ & 1 & 2 & 12 & 0 & 1 & & & \\
\hline 409 & 2 & $10-20$ & 1 & 2 & 1 & 0 & 1 & Chert & & \\
\hline 409 & 2 & $10-20$ & 1 & 2 & 1 & 0 & 1 & & & \\
\hline 409 & 2 & $10-20$ & 1 & 2 & 1 & 0 & 1 & & & \\
\hline 409 & 2 & $10-20$ & 1 & 2 & 1 & 0 & 1 & & & \\
\hline 409 & 2 & $10-20$ & 1 & 2 & 1 & 0 & 1 & & & \\
\hline 409 & 2 & $20-30$ & 6 & 3 & 1 & 0 & 1 & & & \\
\hline 409 & 2 & $20-30$ & 1 & 3 & 1 & 12 & 1 & & & \\
\hline 409 & 2 & $20-30$ & 1 & 4 & 1 & 62 & 1 & & & \\
\hline 409 & 2 & $20-30$ & 1 & 2 & 1 & 0 & 1 & & & \\
\hline 409 & 2 & $20-30$ & 1 & 2 & 1 & 0 & 1 & & & \\
\hline 409 & 2 & $20-30$ & 1 & 2 & 1 & 0 & 1 & & & \\
\hline 409 & 2 & $30-40$ & 1 & 6 & 2 & 12 & 1 & Limestone & & \\
\hline 409 & 2 & $30-40$ & 1 & 2 & 1 & 0 & 1 & & & \\
\hline 409 & 2 & $30-40$ & 1 & 4 & 1 & 0 & 1 & & & \\
\hline 409 & 2 & $30-40$ & 1 & 3 & 1 & 0 & 1 & & & \\
\hline 409 & 2 & $30-40$ & 1 & 3 & 1 & 0 & 1 & & & \\
\hline 409 & 2 & $40-50$ & 1 & 2 & 1 & 0 & 1 & & & \\
\hline 409 & 2 & $40-50$ & 1 & 3 & 1 & 0 & 1 & & & \\
\hline 409 & 2 & $40-50$ & 1 & 5 & 1 & 0 & 1 & & & \\
\hline 409 & 2 & $40-50$ & 1 & 2 & 1 & 0 & 1 & & & \\
\hline 409 & 2 & $40-50$ & 1 & 2 & 1 & 0 & 1 & & & \\
\hline 409 & 2 & $50-60$ & 1 & 4 & 1 & 0 & 1 & Chert & & \\
\hline 409 & 2 & $50-60$ & 1 & 4 & 1 & 0 & 1 & & & \\
\hline 409 & 2 & $50-60$ & 1 & 2 & 1 & 0 & 1 & & & \\
\hline 409 & 2 & $60-70$ & 1 & 2 & 1 & 12 & 1 & Chert \& Limestone & & \\
\hline 409 & 2 & $60-70$ & 1 & 3 & 1 & 12 & 1 & & & \\
\hline 409 & 2 & $60-70$ & 1 & 2 & 1 & 0 & 1 & & & \\
\hline 409 & 2 & $60-70$ & 1 & 2 & 1 & 0 & 1 & & & \\
\hline 409 & 2 & $60-70$ & 1 & 2 & 1 & 0 & 1 & & & \\
\hline 409 & 3 & $20-30$ & 1 & 2 & 1 & 0 & 1 & & & \\
\hline 409 & 3 & $20-30$ & 1 & 2 & 1 & 0 & 1 & & & \\
\hline 409 & 3 & $50-60$ & 1 & 2 & 1 & 100 & 1 & & & \\
\hline 409 & Auger & $0-20$ & 2 & 6 & 4 & 25 & 1 & & & \\
\hline 409 & Auger & $0-20$ & - & - & - & - & - & Chert & & \\
\hline 409 & Auger & $0-20$ & 1 & 4 & 1 & 12 & 1 & & & \\
\hline 409 & Auger & $0-20$ & 1 & 4 & 1 & 0 & 1 & & & \\
\hline 409 & Auger & $0-20$ & 1 & 3 & 1 & 0 & 1 & & & \\
\hline 409 & Auger & $0-20$ & 1 & 3 & 1 & 0 & 1 & & & \\
\hline 409 & Auger & $0-20$ & 1 & 3 & 1 & 0 & 1 & & & \\
\hline 409 & Auger & $120-140$ & 1 & 3 & 1 & 0 & 1 & & & \\
\hline 410 & 1 & $40-50$ & - & - & - & - & - & Limestone & & \\
\hline 410 & 2 & $30-40$ & 7 & - & - & - & - & & & Charcoal \\
\hline 410 & 2 & $30-40$ & 1 & 3 & 1 & 88 & 1 & & & \\
\hline 410 & 3 & $30-40$ & 1 & 4 & 1 & 0 & 1 & & & \\
\hline
\end{tabular}


Table B-1. continued...

\begin{tabular}{|c|c|c|c|c|c|c|c|c|c|c|}
\hline $\begin{array}{r}\text { Site } \\
41 \mathrm{TG} \\
\end{array}$ & $\begin{array}{r}\text { Shovel } \\
\text { Test \# }\end{array}$ & $\begin{array}{c}\text { Depth } \\
\text { cm }\end{array}$ & $\begin{array}{c}\text { Artifact } \\
\text { Type }\end{array}$ & $\begin{array}{l}\text { Max. } \\
\text { Length }\end{array}$ & $\begin{array}{c}\text { Max. } \\
\text { Thickness } \\
\end{array}$ & $\begin{array}{c}\text { Cortex } \\
\% \\
\end{array}$ & $\begin{array}{c}\text { Material } \\
\text { Color }\end{array}$ & $\begin{array}{c}\text { Fire Cracked } \\
\text { Rock }\end{array}$ & $\begin{array}{c}\text { Mussel } \\
\text { Shell }\end{array}$ & $\begin{array}{c}\text { Other / } \\
\text { Remarks }\end{array}$ \\
\hline 410 & 3 & $30-40$ & 1 & 2 & 1 & 12 & 2 & & & \\
\hline 410 & 3 & $30-40$ & 1 & 2 & 1 & 0 & 1 & & & \\
\hline 410 & 3 & $40-50$ & - & - & - & - & - & Limestone & & \\
\hline 411 & 1 & $0-10$ & 1 & 5 & 2 & 12 & 1 & & & \\
\hline 411 & 1 & $10-20$ & 1 & 3 & 1 & 0 & 1 & & & \\
\hline 411 & 1 & $10-20$ & _- & - & - & - & - & Sandstone & & \\
\hline 411 & 1 & $20-30$ & 1 & 7 & 2 & 0 & 1 & & & \\
\hline 411 & 1 & $20-30$ & 1 & 4 & 1 & 12 & 1 & & & \\
\hline 411 & 1 & $30-40$ & 1 & 5 & 2 & 12 & 3 & & & \\
\hline 411 & 1 & $30-40$ & - & - & - & - & - & Limestone & & \\
\hline 411 & 1 & $30-40$ & 1 & 2 & 1 & 0 & 1 & & & \\
\hline 411 & 1 & $30-40$ & 1 & 1 & 1 & 0 & 1 & & & \\
\hline 411 & 1 & $30-40$ & 1 & 2 & 1 & 0 & 1 & & & \\
\hline 411 & 1 & $30-40$ & 1 & 2 & 1 & 0 & 1 & & & \\
\hline 411 & 1 & $30-40$ & 1 & 2 & 1 & 0 & 1 & & & \\
\hline 411 & 1 & $30-40$ & 1 & 1 & 1 & 0 & 1 & & & \\
\hline 411 & 1 & $30-40$ & 1 & 2 & 1 & 0 & 1 & & & \\
\hline 411 & 1 & $60-70$ & 1 & 8 & 2 & 12 & 1 & & & \\
\hline 411 & 2 & $30-40$ & 2 & 4 & 2 & 12 & 1 & Limestone \& Chert & & 10 and 35 fragments \\
\hline 411 & 2 & $30-40$ & 1 & 5 & 2 & 12 & 1 & & & \\
\hline 411 & 2 & $30-40$ & 4 & 4 & 1 & 0 & 1 & & & \\
\hline 411 & 2 & $30-40$ & 1 & 4 & 1 & 0 & 1 & & & \\
\hline 411 & 2 & $30-40$ & 1 & 4 & 2 & 12 & 1 & & & \\
\hline 411 & 2 & $30-40$ & 1 & 2 & 1 & 0 & 1 & & & \\
\hline 411 & 2 & $30-40$ & 1 & 3 & 1 & 0 & 1 & & & \\
\hline 411 & 2 & $30-40$ & 1 & 2 & 1 & 12 & 1 & & & \\
\hline 411 & 2 & $30-40$ & 1 & 4 & 2 & 0 & 1 & & & \\
\hline 411 & 2 & $30-40$ & 1 & 4 & 3 & 12 & 1 & & & \\
\hline 411 & 2 & $30-40$ & 1 & 2 & 1 & 0 & 1 & & & \\
\hline 411 & 2 & $30-40$ & 1 & 2 & 1 & 12 & 1 & & & \\
\hline 411 & 2 & $30-40$ & 1 & 2 & 1 & 0 & 1 & & & \\
\hline 411 & 2 & $30-40$ & 1 & 2 & 1 & 0 & 1 & & & \\
\hline 411 & 2 & $30-40$ & 1 & 2 & 1 & 12 & 1 & & & \\
\hline 411 & 2 & $30-40$ & 1 & 2 & 1 & 0 & 1 & & & \\
\hline 411 & 2 & $30-40$ & 1 & 2 & 1 & 0 & 1 & & & \\
\hline 411 & 2 & $30-40$ & 1 & 1 & 1 & 0 & 1 & & & \\
\hline 411 & 3 & $0-10$ & - & - & - & - & - & Limestone & & \\
\hline 411 & 3 & $10-20$ & 1 & 3 & 1 & 0 & 1 & & $\mathrm{X}$ & \\
\hline 411 & 3 & $10-20$ & 1 & 2 & 1 & 12 & 1 & Limestone \& Other & & $23 \& 18$ fragments \\
\hline 411 & 3 & $10-20$ & 6 & 5 & 1 & 12 & 1 & & & \\
\hline 411 & 3 & $10-20$ & 1 & 4 & 1 & 0 & 1 & & & \\
\hline 411 & 3 & $10-20$ & 1 & 2 & 1 & 12 & 3 & & & \\
\hline 411 & 3 & $10-20$ & 1 & 4 & 1 & 12 & 1 & & & \\
\hline 411 & 3 & $10-20$ & 1 & 3 & 1 & 0 & 1 & & & \\
\hline 411 & 3 & $10-20$ & 1 & 2 & 1 & 0 & 1 & & & \\
\hline 411 & 3 & $10-20$ & 1 & 3 & 1 & 88 & 1 & & & \\
\hline 411 & 3 & $10-20$ & 1 & 3 & 1 & 0 & 2 & & & \\
\hline
\end{tabular}


Table B-1. continued...

\begin{tabular}{|c|c|c|c|c|c|c|c|c|c|c|}
\hline $\begin{array}{r}\text { Site } \\
\text { 41TG } \\
\end{array}$ & $\begin{array}{l}\text { Shovel } \\
\text { Test \# }\end{array}$ & $\begin{array}{c}\text { Depth } \\
\text { cm }\end{array}$ & \begin{tabular}{|c}
$\begin{array}{c}\text { Artifact } \\
\text { Tvpe }\end{array}$ \\
\end{tabular} & $\begin{array}{c}\text { Max. } \\
\text { Length }\end{array}$ & $\begin{array}{c}\text { Max. } \\
\text { Thickness }\end{array}$ & $\begin{array}{c}\text { Cortex } \\
\% \\
\end{array}$ & \begin{tabular}{|c} 
Material \\
Color
\end{tabular} & $\begin{array}{c}\begin{array}{c}\text { Fire Cracked } \\
\text { Rock }\end{array} \\
\end{array}$ & $\begin{array}{c}\text { Mussel } \\
\text { Shell } \\
\end{array}$ & $\begin{array}{c}\text { Other / } \\
\text { Remarks }\end{array}$ \\
\hline 411 & 3 & $10-20$ & 1 & 3 & 1 & 12 & 1 & & & \\
\hline 411 & 3 & $10-20$ & 1 & 2 & 1 & 0 & 2 & & & \\
\hline 411 & 3 & $10-20$ & 1 & 2 & 1 & 0 & 1 & & & \\
\hline 411 & 3 & $10-20$ & 1 & 2 & 1 & 0 & 2 & & & \\
\hline 411 & 3 & $10-20$ & 1 & 2 & 1 & 0 & 1 & & & \\
\hline 411 & 3 & $10-20$ & 1 & 2 & 1 & 0 & 1 & & & \\
\hline 411 & 3 & $10-20$ & 1 & 2 & 1 & 0 & 1 & & & \\
\hline 411 & 3 & $10-20$ & 1 & 2 & 1 & 0 & 1 & & & \\
\hline 411 & 3 & $10-20$ & 1 & 2 & 1 & 0 & 1 & & & \\
\hline 411 & 3 & $10-20$ & 1 & 2 & 1 & 0 & 3 & & & \\
\hline 411 & 3 & $10-20$ & 1 & 2 & 1 & 0 & 1 & & & \\
\hline 411 & 3 & $10-20$ & 1 & 2 & 1 & 0 & 1 & & & \\
\hline 411 & 3 & $10-20$ & 1 & 2 & 1 & 0 & 1 & & & \\
\hline 411 & 3 & $10-20$ & 1 & 7 & 3 & 62 & 1 & & & \\
\hline 411 & 3 & $10-20$ & 1 & 9 & 4 & 38 & 1 & & & \\
\hline 411 & 3 & $20-30$ & 1 & 2 & 1 & 0 & 1 & & & \\
\hline 411 & 3 & $20-30$ & 1 & 2 & 1 & 0 & 1 & & & \\
\hline 411 & 3 & 20-30 & 1 & 2 & 1 & 0 & 1 & & & \\
\hline 411 & 3 & $20-30$ & 1 & 2 & 1 & 0 & 1 & & & \\
\hline 411 & 3 & $20-30$ & 1 & 2 & 1 & 0 & 1 & & & \\
\hline 411 & 3 & $20-30$ & 1 & 2 & 1 & 0 & 1 & & & \\
\hline 411 & 3 & $20-30$ & 1 & 2 & 1 & 0 & 1 & & & \\
\hline 411 & 3 & $20-30$ & 1 & 2 & 1 & 0 & 1 & & & \\
\hline 411 & 3 & $20-30$ & 1 & 1 & 1 & 0 & 1 & & & \\
\hline 411 & 3 & $20-30$ & 1 & 1 & 1 & 0 & 1 & & & \\
\hline 411 & 3 & $20-30$ & 1 & 1 & 1 & 0 & 1 & & & \\
\hline 411 & 3 & $20-30$ & - & - & - & - & - & Limestone & & \\
\hline 412 & 2 & $0-10$ & 1 & 2 & 1 & 0 & 1 & & & \\
\hline 412 & 3 & $10-20$ & 1 & 5 & 1 & 0 & 1 & Limestone & & \\
\hline 412 & 3 & $10-20$ & 1 & 3 & 1 & 12 & 1 & & & \\
\hline 412 & 3 & $10-20$ & 1 & 3 & 1 & 12 & 1 & & & \\
\hline 412 & 3 & $10-20$ & 1 & 2 & 1 & 0 & 1 & & & \\
\hline 412 & 3 & $10-20$ & 1 & 1 & 1 & 38 & 1 & & & \\
\hline 412 & 3 & $10-20$ & 1 & 2 & 1 & 0 & 1 & & & \\
\hline 412 & 4 & $0-10$ & 6 & 6 & 1 & 0 & 1 & & & \\
\hline 412 & 4 & $0-10$ & 4 & 6 & 2 & 0 & 1 & Sandstone & & \\
\hline 412 & 4 & $0-10$ & 1 & 2 & 1 & 0 & 1 & & & \\
\hline 412 & 5 & $0-10$ & 1 & 4 & 2 & 12 & 1 & & & \\
\hline 412 & 6 & $0-10$ & 1 & 2 & 1 & 0 & 1 & & & \\
\hline 412 & 6 & $0-10$ & 1 & 2 & 1 & 0 & 1 & & & \\
\hline 412 & 6 & $0-10$ & 1 & 2 & 1 & 0 & 1 & & & \\
\hline 414 & 1 & $0-10$ & 1 & 4 & 1 & 12 & 1 & & & \\
\hline 414 & 1 & $0-10$ & 1 & 5 & 2 & 100 & 1 & & & \\
\hline 414 & 1 & $0-10$ & 1 & 4 & 1 & 62 & 1 & & & \\
\hline 414 & 1 & $0-10$ & 1 & 3 & 1 & 62 & 1 & & & \\
\hline 414 & 1 & $0-10$ & 1 & 3 & 1 & 100 & 1 & & & \\
\hline 414 & 1 & $0-10$ & 1 & 2 & 1 & 0 & 1 & & & \\
\hline
\end{tabular}


Table B-1. continued...

\begin{tabular}{|c|c|c|c|c|c|c|c|c|c|c|}
\hline $\begin{array}{r}\text { Site } \\
\text { 41TG }\end{array}$ & $\begin{array}{r}\text { Shovel } \\
\text { Test \# }\end{array}$ & $\begin{array}{c}\text { Depth } \\
\text { cm }\end{array}$ & $\begin{array}{c}\text { Artifact } \\
\text { Tvpe }\end{array}$ & $\begin{array}{c}\text { Max. } \\
\text { Length }\end{array}$ & $\begin{array}{c}\text { Max. } \\
\text { Thickness } \\
\end{array}$ & $\begin{array}{c}\text { Cortex } \\
\% \\
\end{array}$ & $\begin{array}{c}\text { Material } \\
\text { Color }\end{array}$ & $\begin{array}{c}\text { Fire Cracked } \\
\text { Rock }\end{array}$ & $\begin{array}{c}\text { Mussel } \\
\text { Shell }\end{array}$ & $\begin{array}{c}\text { Other / } \\
\text { Remarks }\end{array}$ \\
\hline 414 & 1 & $0-10$ & 1 & 3 & 1 & 0 & 1 & & & \\
\hline 414 & 1 & $0-10$ & 1 & 2 & 1 & 12 & 1 & & & \\
\hline 414 & 1 & $0-10$ & 1 & 2 & 1 & 0 & 1 & & & \\
\hline 414 & 1 & $0-10$ & 1 & 2 & 1 & 0 & 1 & & & \\
\hline 414 & 1 & $0-10$ & 1 & 1 & 1 & 0 & 1 & & & \\
\hline 414 & 1 & $0-10$ & 1 & 2 & 1 & 0 & 1 & & & \\
\hline 414 & 1 & $0-10$ & 1 & 2 & 1 & 0 & 1 & & & \\
\hline 414 & 1 & $0-10$ & 1 & 2 & 1 & 0 & 1 & & & \\
\hline 414 & 1 & $0-10$ & 1 & 2 & 1 & 0 & 1 & & & \\
\hline 414 & 1 & $0-10$ & 1 & 2 & 1 & 0 & 1 & & & \\
\hline 414 & 1 & $0-10$ & 1 & 2 & 1 & 0 & 1 & & & \\
\hline 414 & 1 & $0-10$ & 1 & 2 & 1 & 0 & 1 & & & \\
\hline 414 & 1 & $0-10$ & 1 & 1 & 1 & 0 & 1 & & & \\
\hline 414 & 1 & $0-10$ & 1 & 2 & 1 & 100 & 1 & & & \\
\hline 414 & 1 & $0-10$ & 1 & 2 & 1 & 0 & 1 & & & \\
\hline 414 & 1 & $0-10$ & 1 & 1 & 1 & 0 & 1 & & & \\
\hline 414 & 1 & $10-20$ & 1 & 2 & 1 & 100 & 1 & & & \\
\hline 414 & 1 & $10-20$ & 1 & 2 & 1 & 0 & 1 & & & \\
\hline 414 & 1 & $10-20$ & 1 & 2 & 1 & 0 & 1 & & & \\
\hline 414 & 1 & $10-20$ & 1 & 2 & 1 & 0 & 1 & & & \\
\hline 414 & 2 & $0-10$ & 1 & 5 & 1 & 12 & 1 & Limestone, Chert & $\underline{X}$ & \\
\hline 414 & 2 & $0-10$ & 1 & 4 & 2 & 12 & 1 & & & \\
\hline 414 & 2 & $0-10$ & 6 & 5 & 2 & 12 & 1 & & & \\
\hline 414 & 2 & $0-10$ & 1 & 4 & 1 & 12 & 1 & & & \\
\hline 414 & 2 & $0-10$ & 1 & 3 & 2 & 88 & 1 & & & \\
\hline 414 & 2 & $0-10$ & 6 & 3 & 1 & 0 & 1 & & & \\
\hline 414 & 2 & $0-10$ & 1 & 3 & 1 & 0 & 1 & & & \\
\hline 414 & 2 & $0-10$ & 6 & 3 & 1 & 62 & 1 & & & \\
\hline 414 & 2 & $0-10$ & 1 & 3 & 1 & 0 & 1 & & & \\
\hline 414 & 2 & $0-10$ & 1 & 2 & 1 & 0 & 1 & & & \\
\hline 414 & 2 & $0-10$ & 1 & 3 & 1 & 38 & 1 & & & \\
\hline 414 & 2 & $0-10$ & 1 & 3 & 1 & 0 & 1 & & & \\
\hline 414 & 2 & $0-10$ & 1 & 2 & 1 & 0 & 1 & & & \\
\hline 414 & 2 & $0-10$ & 1 & 2 & 1 & 0 & 1 & & & \\
\hline 414 & 2 & $0-10$ & 1 & 2 & 1 & 12 & 1 & & & \\
\hline 414 & 2 & $0-10$ & 1 & 2 & 1 & 0 & 1 & & & \\
\hline 414 & 2 & $0-10$ & 6 & 3 & 1 & 0 & 1 & & & \\
\hline 414 & 2 & $0-10$ & 1 & 2 & 1 & 0 & 1 & & & \\
\hline 414 & 2 & $0-10$ & 1 & 2 & 1 & 0 & 1 & & & \\
\hline 414 & 2 & $0-10$ & 1 & 2 & 1 & 12 & 1 & & & \\
\hline 414 & 2 & $0-10$ & 1 & 2 & 1 & 0 & 1 & & & \\
\hline 414 & 2 & $0-10$ & 1 & 2 & 1 & 0 & 1 & & & \\
\hline 414 & 2 & $0-10$ & 1 & 2 & 1 & 0 & 1 & & & \\
\hline 414 & 2 & $0-10$ & 6 & 3 & 1 & 0 & 1 & & & Blade \\
\hline 414 & 2 & $0-10$ & 4 & 2 & 1 & 0 & 1 & & & Distal end \\
\hline 414 & 2 & $0-10$ & 1 & 2 & 1 & 0 & 1 & & & \\
\hline 414 & 2 & $0-10$ & 1 & 1 & 1 & 0 & 1 & & & \\
\hline
\end{tabular}


Table B-1. continued...

\begin{tabular}{|c|c|c|c|c|c|c|c|c|c|c|}
\hline $\begin{array}{r}\text { Site } \\
41 \mathrm{TG} \\
\end{array}$ & $\begin{array}{l}\text { Shovel } \\
\text { Test \# }\end{array}$ & $\begin{array}{c}\text { Depth } \\
\text { cm }\end{array}$ & $\begin{array}{c}\text { Artifact } \\
\text { Type }\end{array}$ & $\begin{array}{c}\text { Max. } \\
\text { Length }\end{array}$ & $\begin{array}{c}\text { Max. } \\
\text { Thickness } \\
\end{array}$ & $\begin{array}{c}\text { Cortex } \\
\% \\
\end{array}$ & \begin{tabular}{|c} 
Material \\
Color
\end{tabular} & $\begin{array}{c}\text { Fire Cracked } \\
\text { Rock }\end{array}$ & $\begin{array}{c}\text { Mussel } \\
\text { Shell }\end{array}$ & $\begin{array}{c}\text { Other } / \\
\text { Remarks }\end{array}$ \\
\hline 414 & 2 & $0-10$ & 1 & 2 & 1 & 0 & 1 & & & \\
\hline 414 & 2 & $0-10$ & 1 & 2 & 1 & 38 & 1 & & & \\
\hline 414 & 2 & $0-10$ & 1 & 2 & 1 & 0 & 1 & & & \\
\hline 414 & 2 & $0-10$ & 1 & 2 & 1 & 0 & 3 & & & \\
\hline 414 & 2 & $0-10$ & 1 & 2 & 1 & 0 & 1 & & & \\
\hline 414 & 2 & $0-10$ & 1 & 2 & 1 & 0 & 1 & & & \\
\hline 414 & 2 & $0-10$ & 1 & 2 & 1 & 0 & 1 & & & \\
\hline 414 & 2 & $10-20$ & 1 & 5 & 2 & 0 & 1 & & & \\
\hline 414 & 2 & $10-20$ & 1 & 2 & 1 & 0 & 1 & & & \\
\hline 414 & 2 & $10-20$ & 1 & 2 & 1 & 0 & 1 & & & \\
\hline 414 & 2 & $10-20$ & 6 & 3 & 1 & 88 & 1 & & & \\
\hline 414 & 2 & $10-20$ & 1 & 2 & 1 & 0 & 1 & & & \\
\hline 414 & 2 & $10-20$ & 1 & 3 & 1 & 88 & 1 & & & \\
\hline 414 & 2 & $10-20$ & 1 & 2 & 1 & 0 & 1 & & & \\
\hline 414 & 2 & $10-20$ & 1 & 3 & 1 & 0 & 1 & & & \\
\hline 414 & 2 & $10-20$ & 1 & 2 & 1 & 0 & 1 & & & \\
\hline 414 & 2 & $10-20$ & 6 & 3 & 1 & 0 & 1 & & & \\
\hline 414 & 2 & $10-20$ & 1 & 2 & 1 & 0 & 3 & & & \\
\hline 414 & 2 & $10-20$ & 1 & 2 & 1 & 0 & 1 & & & \\
\hline 414 & 2 & $10-20$ & 1 & 2 & 1 & 0 & 1 & & & \\
\hline 414 & 2 & $10-20$ & 1 & 2 & 1 & 0 & 1 & & & \\
\hline 414 & 2 & $10-20$ & 1 & 2 & 1 & 0 & 1 & & & \\
\hline 414 & 2 & $10-20$ & 1 & 2 & 1 & 0 & 1 & & & \\
\hline 414 & 2 & $10-20$ & 1 & 2 & 1 & 0 & 1 & & & \\
\hline 414 & 2 & $10-20$ & 1 & 3 & 1 & 0 & 3 & & & \\
\hline 414 & 2 & $10-20$ & 1 & 2 & 1 & 0 & 1 & & & \\
\hline 414 & 2 & $10-20$ & 1 & 2 & 1 & 0 & 1 & & & \\
\hline 414 & 2 & $10-20$ & 1 & 2 & 1 & 0 & 1 & & & \\
\hline 414 & 2 & $10-20$ & 1 & 2 & 1 & 0 & 1 & & & \\
\hline 414 & 2 & $10-20$ & 1 & 2 & 1 & 0 & 1 & & & \\
\hline 414 & 2 & $10-20$ & 1 & 2 & 1 & 12 & 1 & & & \\
\hline 414 & 2 & $10-20$ & 1 & 2 & 1 & 38 & 1 & & & \\
\hline 414 & 2 & $10-20$ & 1 & 3 & 1 & 12 & 3 & & & \\
\hline 414 & 2 & $20-30$ & 6 & 3 & 1 & 0 & 1 & Limestone & & \\
\hline 414 & 2 & $20-30$ & 1 & 4 & 1 & 12 & 1 & & & \\
\hline 414 & 2 & $20-30$ & 1 & 5 & 2 & 12 & 1 & & & \\
\hline 414 & 2 & $20-30$ & 1 & 2 & 1 & 12 & 1 & & & \\
\hline 414 & 2 & $20-30$ & 1 & 2 & 1 & 0 & 3 & & & \\
\hline 414 & 2 & $20-30$ & 1 & 2 & 1 & 0 & 1 & & & \\
\hline 414 & 2 & $20-30$ & 1 & 2 & 1 & 0 & 1 & & & \\
\hline 414 & 3 & $0-10$ & 1 & 4 & 1 & 12 & 1 & Chert & & \\
\hline 414 & 3 & $0-10$ & 6 & 6 & 2 & 12 & 1 & & & \\
\hline 414 & 3 & $0-10$ & 1 & 3 & 1 & 12 & 1 & & & \\
\hline 414 & 3 & $0-10$ & 1 & 3 & 1 & 88 & 1 & & & \\
\hline 414 & 3 & $0-10$ & 1 & 3 & 1 & 12 & 1 & & & \\
\hline 414 & 3 & $0-10$ & 6 & 3 & 1 & 0 & 1 & & & \\
\hline 414 & 3 & $0-10$ & 1 & 2 & 1 & 0 & 1 & & & \\
\hline
\end{tabular}


Table B-1. continued...

\begin{tabular}{|c|c|c|c|c|c|c|c|c|c|c|}
\hline $\begin{array}{r}\text { Site } \\
\text { 41TG } \\
\end{array}$ & $\begin{array}{l}\text { Shovel } \\
\text { Test \# }\end{array}$ & $\begin{array}{c}\text { Depth } \\
\mathrm{cm}\end{array}$ & $\begin{array}{c}\text { Artifact } \\
\text { Type }\end{array}$ & $\begin{array}{c}\text { Max. } \\
\text { Length }\end{array}$ & $\begin{array}{c}\text { Max. } \\
\text { Thickness }\end{array}$ & $\begin{array}{c}\text { Cortex } \\
\%\end{array}$ & $\begin{array}{c}\text { Material } \\
\text { Color }\end{array}$ & $\begin{array}{c}\text { Fire Cracked } \\
\text { Rock }\end{array}$ & $\begin{array}{c}\text { Mussel } \\
\text { Shell }\end{array}$ & $\begin{array}{r}\text { Other / } \\
\text { Remarks }\end{array}$ \\
\hline 414 & 3 & $0-10$ & 1 & 1 & 1 & 88 & 1 & & & \\
\hline 414 & 3 & $0-10$ & 1 & 2 & 1 & 0 & 1 & & & \\
\hline 414 & 3 & $0-10$ & 1 & 2 & 1 & 0 & 1 & & & \\
\hline 414 & 3 & $0-10$ & 1 & 2 & 1 & 0 & 1 & & & \\
\hline 414 & 3 & $0-10$ & 1 & 2 & 1 & 0 & 1 & & & \\
\hline 414 & 3 & $0-10$ & 1 & 2 & 1 & 0 & 1 & & & \\
\hline 414 & 3 & $0-10$ & 1 & 2 & 1 & 0 & 1 & & & \\
\hline 414 & 3 & $0-10$ & 1 & 2 & 1 & 0 & 1 & & & \\
\hline 414 & 3 & $0-10$ & 1 & 2 & 1 & 0 & 1 & & & \\
\hline 414 & 3 & $0-10$ & 1 & 2 & 1 & 0 & 1 & & & \\
\hline 414 & 3 & $0-10$ & 1 & 2 & 1 & 0 & 1 & & & \\
\hline 414 & 3 & $0-10$ & 1 & 2 & 1 & 0 & 3 & & & \\
\hline 414 & 3 & $10-20$ & 1 & 4 & 1 & 12 & 1 & & & \\
\hline 414 & 3 & $10-20$ & 1 & 2 & 1 & 0 & 1 & & & \\
\hline 414 & 3 & $10-20$ & 1 & 2 & 1 & 0 & 1 & & & \\
\hline 414 & 3 & $10-20$ & 1 & 2 & 1 & 0 & 1 & & & \\
\hline 414 & 3 & $10-20$ & 1 & 1 & 1 & 12 & 1 & & & \\
\hline 414 & 3 & $10-20$ & 1 & 2 & 1 & 0 & 1 & & & \\
\hline 414 & 3 & $10-20$ & 1 & 2 & 1 & 0 & 1 & & & \\
\hline 414 & 3 & $10-20$ & 1 & 2 & 1 & 0 & 1 & & & \\
\hline 414 & 3 & $10-20$ & 1 & 2 & 1 & 0 & 1 & & & \\
\hline 414 & 3 & $10-20$ & 1 & 2 & 1 & 0 & 1 & & & \\
\hline 414 & 3 & $10-20$ & 1 & 2 & 1 & 0 & 1 & & & \\
\hline 414 & 3 & $10-20$ & 1 & 3 & 1 & 0 & 1 & & & \\
\hline 414 & 3 & $10-20$ & 1 & 2 & 1 & 0 & 3 & & & \\
\hline 414 & 3 & $20-30$ & 1 & 2 & 1 & 0 & 1 & Limestone & & \\
\hline 414 & 3 & $20-30$ & 1 & 3 & 1 & 0 & 1 & & & \\
\hline 414 & 3 & $20-30$ & 1 & 3 & 1 & 0 & 3 & & & \\
\hline 414 & 3 & $20-30$ & 6 & 7 & 4 & 12 & 1 & & & \\
\hline 414 & 3 & $20-30$ & 1 & 5 & 1 & 0 & 1 & & & \\
\hline 414 & 3 & $20-30$ & 1 & 4 & 1 & 12 & 1 & & & \\
\hline 414 & 3 & $20-30$ & 1 & 2 & 1 & 0 & 1 & & & \\
\hline 414 & 3 & $30-40$ & 1 & 2 & 1 & 0 & 3 & & & \\
\hline 414 & 3 & $30-40$ & 1 & 3 & 1 & 0 & 1 & & & \\
\hline 414 & 3 & $30-40$ & 1 & 2 & 1 & 0 & 1 & & & \\
\hline 414 & 3 & $30-40$ & 1 & 2 & 1 & 0 & 1 & & & \\
\hline 414 & 3 & $30-40$ & 1 & 2 & 1 & 0 & 1 & & & \\
\hline 414 & 3 & $30-40$ & 1 & 2 & 1 & 0 & 1 & & & \\
\hline 414 & 3 & $30-40$ & 1 & 2 & 1 & 12 & 1 & & & \\
\hline 414 & 3 & $40-50$ & 1 & 4 & 1 & 0 & 1 & & & \\
\hline 416 & 2 & $10-20$ & 1 & 5 & 1 & 88 & 1 & & & \\
\hline 416 & 4 & $10-20$ & 1 & 5 & 2 & 88 & 1 & & & \\
\hline 416 & 4 & $20-30$ & 1 & 2 & 1 & 0 & 2 & Chert & & \\
\hline 417 & 1 & $30-40$ & 1 & 6 & 2 & 88 & 1 & & & \\
\hline 418 & 2 & $10-20$ & 1 & 1 & 1 & 0 & 1 & & & \\
\hline 418 & 2 & & 1 & 2 & 1 & 62 & 1 & & & \\
\hline 419 & 1 & $0-10$ & 1 & 3 & 1 & 0 & 2 & & & \\
\hline
\end{tabular}


Table B-1. continued...

\begin{tabular}{|c|c|c|c|c|c|c|c|c|c|c|}
\hline $\begin{array}{r}\text { Site } \\
\text { 41TG } \\
\end{array}$ & $\begin{array}{l}\text { Shovel } \\
\text { Test \# }\end{array}$ & $\begin{array}{c}\text { Depth } \\
\text { cm }\end{array}$ & \begin{tabular}{|c}
$\begin{array}{c}\text { Artifact } \\
\text { Tvpe }\end{array}$ \\
\end{tabular} & $\begin{array}{c}\text { Max. } \\
\text { Length }\end{array}$ & $\begin{array}{c}\text { Max. } \\
\text { Thickness }\end{array}$ & $\begin{array}{c}\text { Cortex } \\
\% \\
\end{array}$ & \begin{tabular}{|c} 
Material \\
Color
\end{tabular} & $\begin{array}{c}\text { Fire Cracked } \\
\text { Rock }\end{array}$ & $\begin{array}{c}\text { Mussel } \\
\text { Shell } \\
\end{array}$ & $\begin{array}{c}\text { Other / } \\
\text { Remarks }\end{array}$ \\
\hline 419 & 2 & $0-10$ & 1 & 3 & 1 & 100 & 1 & & & \\
\hline 419 & 2 & $0-10$ & 1 & 2 & 1 & 0 & 1 & & & \\
\hline 419 & 2 & $10-20$ & 1 & 2 & 1 & 0 & 1 & Chert & & \\
\hline 419 & 6 & $0-10$ & 1 & 4 & 1 & 88 & 1 & & & \\
\hline 419 & 9 & $0-10$ & 1 & 3 & 1 & 0 & 2 & Chert & & \\
\hline 419 & 9 & $0-10$ & 1 & 5 & 2 & 38 & 2 & & & \\
\hline 419 & 9 & $0-10$ & 1 & 2 & 1 & 0 & 1 & & & \\
\hline 419 & 9 & $0-10$ & 6 & 3 & 1 & 88 & 1 & & & \\
\hline 419 & 9 & $0-10$ & 1 & 3 & 1 & 0 & 1 & & & \\
\hline 419 & 9 & $0-10$ & 5 & 5 & 2 & 88 & 1 & & & \\
\hline 419 & 9 & $0-10$ & 2 & 7 & 3 & 25 & 1 & & & \\
\hline 419 & 9 & $0-10$ & 6 & 4 & 2 & 62 & 1 & & & \\
\hline 424 & 1 & $10-20$ & 4 & 2 & 1 & 0 & 1 & & & \\
\hline 424 & 2 & $10-20$ & 1 & 2 & 1 & 88 & 3 & & & \\
\hline 424 & 2 & $50-60$ & 1 & 5 & 2 & 0 & 1 & & & \\
\hline 424 & 2 & $50-60$ & 1 & 4 & 2 & 38 & 1 & & & \\
\hline 424 & 3 & $50-60$ & 6 & 3 & 1 & 0 & 1 & & & \\
\hline 424 & 7 & $0-10$ & - & - & - & - & - & Limestone \& Other & & \\
\hline 424 & 7 & $60-70$ & - & - & - & - & - & Sandstone & & \\
\hline 424 & 8 & $10-20$ & - & - & - & - & - & Limestone & & \\
\hline 424 & 13 & $0-10$ & 6 & 5 & 2 & 12 & 1 & & & Finely flaked \\
\hline 424 & 13 & $0-10$ & 1 & 2 & 1 & 12 & 1 & & & \\
\hline 424 & 14 & $0-10$ & - & - & - & - & - & Limestone \& Chert & & \\
\hline 426 & 1 & $50-60$ & 1 & 4 & 1 & 0 & 1 & & & \\
\hline 426 & 1 & $50-60$ & 1 & 2 & 1 & 0 & 1 & & & \\
\hline 426 & 1 & $50-60$ & - & - & - & - & - & & $\mathrm{X}$ & Bird bone fragments \\
\hline 426 & 2 & $0-10$ & - & - & - & - & - & & $\mathrm{X}$ & Bone fragments, Charcoal \\
\hline 426 & 2 & $10-20$ & 7 & - & - & - & - & & & Charcoal \\
\hline 426 & 4 & $0-10$ & - & - & - & - & - & Limestone & $\mathrm{X}$ & \\
\hline 426 & 5 & $20-30$ & 1 & 2 & 1 & 0 & 1 & & & \\
\hline 426 & 5 & $20-30$ & 1 & 2 & 1 & 0 & 1 & & & \\
\hline 426 & 6 & $0-10$ & 1 & 2 & 1 & 0 & 3 & & & \\
\hline 426 & 6 & $0-10$ & 7 & - & - & - & - & & $\mathrm{X}$ & \\
\hline 426 & 6 & $0-10$ & 1 & 1 & 1 & 0 & 1 & & & \\
\hline 426 & 6 & $0-10$ & 1 & 2 & 1 & 0 & 1 & & & \\
\hline 426 & 6 & $0-10$ & 1 & 1 & 1 & 0 & 1 & & & \\
\hline 426 & 6 & $0-10$ & 1 & 2 & 1 & 0 & 3 & & & \\
\hline 426 & 7 & $0-10$ & 1 & 3 & 1 & 0 & 1 & Limestone \& Chert & & Charcoal \\
\hline 426 & 7 & $0-10$ & 1 & 3 & 1 & 0 & 1 & & & \\
\hline 426 & 7 & $0-10$ & 1 & 1 & 1 & 0 & 1 & & & \\
\hline 426 & 7 & $30-40$ & 1 & 2 & 1 & 100 & 1 & & & \\
\hline 427 & 2 & $0-10$ & 1 & 3 & 1 & 12 & 1 & & & \\
\hline 427 & 2 & $0-10$ & 1 & 4 & 1 & 12 & 1 & & & \\
\hline 427 & 2 & $0-10$ & 1 & 2 & 1 & 0 & 1 & & & \\
\hline 427 & 2 & $0-10$ & 1 & 2 & 1 & 0 & 1 & & & \\
\hline 427 & 2 & $0-10$ & 1 & 2 & 1 & 12 & 1 & & & \\
\hline 427 & 2 & $0-10$ & 1 & 1 & 1 & 0 & 1 & & & \\
\hline
\end{tabular}


Table B-1. continued...

\begin{tabular}{|c|c|c|c|c|c|c|c|c|c|c|}
\hline $\begin{array}{r}\text { Site } \\
41 \mathrm{TG} \\
\end{array}$ & $\begin{array}{l}\text { Shovel } \\
\text { Test \# }\end{array}$ & $\begin{array}{c}\text { Depth } \\
\text { cm }\end{array}$ & $\begin{array}{c}\text { Artifact } \\
\text { Tvpe }\end{array}$ & $\begin{array}{l}\text { Max. } \\
\text { Length }\end{array}$ & $\begin{array}{c}\text { Max. } \\
\text { Thickness }\end{array}$ & $\begin{array}{c}\text { Cortex } \\
\% \\
\end{array}$ & $\begin{array}{c}\text { Material } \\
\text { Color }\end{array}$ & $\begin{array}{c}\text { Fire Cracked } \\
\text { Rock } \\
\end{array}$ & $\begin{array}{c}\text { Mussel } \\
\text { Shell }\end{array}$ & $\begin{array}{c}\text { Other / } \\
\text { Remarks }\end{array}$ \\
\hline 427 & 2 & $0-10$ & 1 & 2 & 1 & 12 & 1 & & & \\
\hline 427 & 2 & $0-10$ & 1 & 2 & 1 & 0 & 1 & & & \\
\hline 427 & 2 & $0-10$ & 1 & 2 & 1 & 12 & 1 & & & \\
\hline 427 & 2 & $0-10$ & 1 & 2 & 1 & 0 & 1 & & & \\
\hline 427 & 2 & $0-10$ & - & - & - & - & - & Limestone & & \\
\hline 427 & 2 & $0-10$ & - & - & - & - & - & Sandstone & & \\
\hline 427 & 3 & $0-10$ & 1 & 3 & 1 & 12 & 1 & & & \\
\hline 427 & 3 & $0-10$ & 1 & 2 & 1 & 0 & 1 & & & \\
\hline 427 & 3 & $0-10$ & 1 & 2 & 1 & 0 & 1 & & & \\
\hline 427 & 3 & $0-10$ & 1 & 2 & 1 & 0 & 1 & & & \\
\hline 427 & 3 & $0-10$ & - & - & - & - & - & Chert & & \\
\hline 427 & 3 & $10-20$ & 1 & 2 & 1 & 100 & 1 & & & \\
\hline 427 & 4 & $0-10$ & 1 & 2 & 1 & 0 & 1 & & & \\
\hline 427 & 4 & $0-10$ & 1 & 1 & 1 & 0 & 1 & & & \\
\hline 427 & 4 & $0-10$ & 1 & 1 & 1 & 0 & 1 & & & \\
\hline 427 & 4 & $10-20$ & 1 & 3 & 1 & 0 & 4 & & & \\
\hline 427 & 4 & $10-20$ & 7 & - & - & - & - & & $x$ & \\
\hline 427 & 4 & $20-30$ & 1 & 4 & 1 & 0 & 1 & & & \\
\hline 427 & 5 & $0-10$ & - & - & - & - & - & Limestone & & \\
\hline 427 & 5 & $10-20$ & - & - & - & - & - & Chert & & \\
\hline 427 & 5 & $10-20$ & - & - & - & - & - & Limestone & & \\
\hline 427 & 5 & $10-20$ & - & - & - & - & - & & $x$ & \\
\hline 427 & 5 & $10-20$ & - & - & - & - & - & Sandstone & & \\
\hline 427 & 5 & $10-20$ & 1 & 2 & 1 & 0 & 1 & & & \\
\hline 427 & 5 & $30-40$ & 1 & 6 & 1 & 88 & 1 & & & \\
\hline 427 & 5 & $30-40$ & 1 & 2 & 1 & 12 & 1 & & & \\
\hline 427 & 5 & $30-40$ & 1 & 2 & 1 & 0 & 3 & & & \\
\hline 427 & 5 & $30-40$ & - & - & - & - & - & & & Chert potlid \\
\hline 427 & 5 & $30-40$ & - & - & - & - & - & Limestone & & \\
\hline 427 & 5 & $40-48$ & 1 & 4 & 1 & 12 & 1 & & & \\
\hline 427 & 5 & $40-48$ & 1 & 2 & 1 & 0 & 4 & & & \\
\hline 427 & 5 & $40-48$ & 6 & 4 & 1 & 12 & 1 & & & \\
\hline 427 & 5 & $40-48$ & 1 & 4 & 1 & 0 & 1 & & & Blade \\
\hline 427 & 5 & $40-48$ & 1 & 2 & 1 & 88 & 1 & & & \\
\hline 427 & 5 & $40-48$ & 1 & 2 & 1 & 0 & 1 & & & \\
\hline 427 & 5 & $40-48$ & 1 & 2 & 1 & 0 & 1 & & & \\
\hline 427 & 5 & $40-48$ & 1 & 2 & 1 & 12 & 1 & & & \\
\hline 427 & 5 & $40-48$ & 1 & 2 & 1 & 0 & 1 & & & \\
\hline 427 & 5 & $40-48$ & 1 & 2 & 1 & 12 & 4 & & & \\
\hline 427 & 5 & $40-48$ & 1 & 2 & 1 & 0 & 1 & & & \\
\hline 427 & 5 & $40-48$ & 1 & 2 & 1 & 0 & 1 & & & \\
\hline 427 & 5 & $40-48$ & - & - & - & - & - & & $X$ & \\
\hline 427 & 6 & $0-10$ & 6 & 5 & 1 & 88 & 1 & & & \\
\hline 427 & 6 & $0-10$ & 6 & 5 & 2 & 38 & 1 & & & \\
\hline 427 & 6 & $0-10$ & 1 & 5 & 1 & 12 & 1 & & & \\
\hline 427 & 6 & $0-10$ & 1 & 4 & 2 & 12 & 1 & & & \\
\hline 427 & 6 & $0-10$ & 1 & 4 & 2 & 12 & 1 & & & \\
\hline
\end{tabular}


Table B-1. continued...

\begin{tabular}{|c|c|c|c|c|c|c|c|c|c|c|}
\hline $\begin{array}{r}\text { Site } \\
41 \mathrm{TG} \\
\end{array}$ & $\begin{array}{l}\text { Shovel } \\
\text { Test \# }\end{array}$ & $\begin{array}{c}\text { Depth } \\
\text { cm }\end{array}$ & $\begin{array}{c}\text { Artifact } \\
\text { Type }\end{array}$ & $\begin{array}{c}\text { Max. } \\
\text { Length }\end{array}$ & $\begin{array}{c}\text { Max. } \\
\text { Thickness } \\
\end{array}$ & $\begin{array}{c}\text { Cortex } \\
\% \\
\end{array}$ & \begin{tabular}{|c} 
Material \\
Color
\end{tabular} & $\begin{array}{c}\text { Fire Cracked } \\
\text { Rock }\end{array}$ & $\begin{array}{c}\text { Mussel } \\
\text { Shell }\end{array}$ & $\begin{array}{c}\text { Other } / \\
\text { Remarks }\end{array}$ \\
\hline 427 & 6 & $0-10$ & 1 & 3 & 1 & 100 & 1 & & & \\
\hline 427 & 6 & $0-10$ & 1 & 3 & 1 & 88 & 1 & & & \\
\hline 427 & 6 & $0-10$ & 6 & 3 & 1 & 0 & 1 & & & \\
\hline 427 & 6 & $0-10$ & 6 & 4 & 1 & 12 & 1 & & & \\
\hline 427 & 6 & $0-10$ & 1 & 2 & 1 & 12 & 3 & & & \\
\hline 427 & 6 & $0-10$ & 1 & 2 & 1 & 0 & 1 & & & \\
\hline 427 & 6 & $0-10$ & 1 & 2 & 1 & 88 & 1 & & & \\
\hline 427 & 6 & $0-10$ & 1 & 2 & 1 & 0 & 1 & & & \\
\hline 427 & 6 & $0-10$ & 1 & 1 & 1 & 0 & 1 & & & \\
\hline 427 & 6 & $0-10$ & 1 & 2 & 1 & 0 & 1 & & & \\
\hline 427 & 6 & $0-10$ & 1 & 1 & 1 & 0 & 1 & & & \\
\hline 427 & 6 & $0-10$ & 1 & 1 & 1 & 0 & 1 & & & \\
\hline 427 & 6 & $0-10$ & - & - & - & - & - & Limestone & & \\
\hline 427 & 6 & $0-10$ & - & - & - & - & - & Chert & & \\
\hline 427 & 6 & $10-20$ & 6 & 5 & 2 & 12 & 1 & & & \\
\hline 427 & 6 & $10-20$ & 6 & 7 & 1 & 88 & 1 & & & \\
\hline 427 & 6 & $10-20$ & 1 & 3 & 1 & 0 & 1 & & & \\
\hline 427 & 6 & $10-20$ & 1 & 4 & 1 & 12 & 1 & & & \\
\hline 427 & 6 & $10-20$ & 1 & 3 & 1 & 100 & 4 & & & \\
\hline 427 & 6 & $10-20$ & 6 & 3 & 1 & 12 & 1 & & & \\
\hline 427 & 6 & $10-20$ & 1 & 3 & 1 & 12 & 1 & & & \\
\hline 427 & 6 & $10-20$ & 7 & - & - & - & - & & $\mathrm{X}$ & \\
\hline 427 & 6 & $10-20$ & - & _. & - & - & _. & Limestone & & \\
\hline 427 & 6 & $10-20$ & - & - & - & - & - & Sandstone & & \\
\hline 427 & 7 & $0-10$ & 6 & 3 & 1 & 0 & 1 & & & \\
\hline 427 & 7 & $0-10$ & 7 & - & - & - & - & & $\mathrm{x}$ & \\
\hline 427 & 7 & $0-10$ & 1 & 1 & 1 & 12 & 1 & & & \\
\hline 427 & 8 & $0-10$ & - & - & - & - & - & Limestone & & \\
\hline 427 & 8 & $0-10$ & 4 & 6 & 3 & 0 & 1 & & & \\
\hline 427 & 8 & $0-10$ & - & - & - & - & - & Chert & & \\
\hline 427 & 8 & $0-10$ & 1 & 3 & 1 & 0 & 1 & & & \\
\hline 427 & 8 & $0-10$ & 1 & 3 & 1 & 12 & 1 & & & \\
\hline 427 & 8 & $0-10$ & 1 & 2 & 1 & 0 & 1 & & & \\
\hline 427 & 8 & $0-10$ & 1 & 2 & 1 & 12 & 1 & & & \\
\hline 427 & 8 & $0-10$ & 1 & 3 & 1 & 0 & 1 & & & \\
\hline 427 & 8 & $0-10$ & 1 & 3 & 1 & 0 & 1 & & & \\
\hline 427 & 8 & $0-10$ & 1 & 2 & 1 & 12 & 1 & & & \\
\hline 427 & 8 & $0-10$ & 1 & 2 & 1 & 0 & 1 & & & \\
\hline 427 & 8 & $0-10$ & 1 & 3 & 1 & 0 & 3 & & & \\
\hline 427 & 8 & $0-10$ & 1 & 2 & 1 & 0 & 1 & & & \\
\hline 427 & 8 & $0-10$ & 1 & 2 & 1 & 0 & 1 & & & \\
\hline 427 & 8 & $0-10$ & 1 & 1 & 3 & 12 & 1 & & & \\
\hline 427 & 8 & $0-10$ & 1 & 2 & 1 & 0 & 1 & & & \\
\hline 427 & 8 & $0-10$ & 1 & 1 & 1 & 0 & 1 & & & \\
\hline 427 & 8 & $0-10$ & 1 & 2 & 1 & 0 & 1 & & & \\
\hline 427 & 8 & $0-10$ & 1 & 1 & 1 & 0 & 1 & & & \\
\hline 427 & 8 & $0-10$ & 1 & 1 & 1 & 12 & 1 & & & \\
\hline
\end{tabular}


Table B-1. continued...

\begin{tabular}{|c|c|c|c|c|c|c|c|c|c|c|}
\hline $\begin{array}{r}\text { Site } \\
\text { 41TG } \\
\end{array}$ & $\begin{array}{r}\text { Shovel } \\
\text { Test \# }\end{array}$ & $\begin{array}{c}\text { Depth } \\
\text { cm }\end{array}$ & $\begin{array}{c}\text { Artifact } \\
\text { Tvpe }\end{array}$ & $\begin{array}{c}\text { Max. } \\
\text { Length }\end{array}$ & $\begin{array}{c}\text { Max. } \\
\text { Thickness } \\
\end{array}$ & $\begin{array}{c}\text { Cortex } \\
\% \\
\end{array}$ & $\begin{array}{c}\text { Material } \\
\text { Color }\end{array}$ & $\begin{array}{c}\text { Fire Cracked } \\
\text { Rock }\end{array}$ & $\begin{array}{c}\text { Mussel } \\
\text { Shell }\end{array}$ & $\begin{array}{r}\text { Other / } \\
\text { Remarks }\end{array}$ \\
\hline 427 & 8 & $0-10$ & 1 & 2 & 1 & 38 & 1 & & & \\
\hline 427 & 8 & $0-10$ & 1 & 2 & 1 & 62 & 3 & & & \\
\hline 427 & 8 & $0-10$ & 1 & 2 & 1 & 0 & 1 & & & \\
\hline 427 & 8 & $0-10$ & 1 & 2 & 1 & 100 & 1 & & & \\
\hline 427 & 8 & $0-10$ & 1 & 2 & 1 & 12 & 1 & & & \\
\hline 427 & 8 & $0-10$ & 1 & 2 & 1 & 0 & 4 & & & \\
\hline 427 & 8 & $0-10$ & 1 & 3 & 1 & 100 & 4 & & & \\
\hline 427 & 8 & $0-10$ & 1 & 2 & 1 & 0 & 1 & & & \\
\hline 427 & 8 & $0-10$ & 1 & 2 & 1 & 12 & 1 & & & \\
\hline 427 & 8 & $0-10$ & 1 & 1 & 1 & 12 & 1 & & & \\
\hline 427 & 8 & $0-10$ & 1 & 2 & 1 & 0 & 1 & & & \\
\hline 427 & 8 & $0-10$ & 1 & 2 & 1 & 12 & 3 & & & \\
\hline 427 & 8 & $0-10$ & 1 & 1 & 1 & 12 & 1 & & & \\
\hline 427 & 8 & $0-10$ & 1 & 2 & 1 & 38 & 1 & & & \\
\hline 427 & 8 & $0-10$ & 1 & 1 & 1 & 100 & 4 & & & \\
\hline 427 & 8 & $0-10$ & 1 & 1 & 1 & 100 & 1 & & & \\
\hline 427 & 8 & $0-10$ & 1 & 1 & 1 & 0 & 1 & & & \\
\hline 427 & 8 & $0-10$ & 1 & 1 & 1 & 0 & 1 & & & \\
\hline 427 & 8 & $0-10$ & 1 & 2 & 1 & 0 & 1 & & & \\
\hline 427 & 8 & $0-10$ & 1 & 2 & 1 & 0 & 1 & & & \\
\hline 427 & 8 & $0-10$ & 1 & 2 & 1 & 0 & 1 & & & \\
\hline 427 & 8 & $0-10$ & 1 & 2 & 1 & 0 & 1 & & & \\
\hline 427 & 8 & $0-10$ & 1 & 1 & 1 & 0 & 1 & & & \\
\hline 427 & 8 & $0-10$ & 1 & 1 & 1 & 0 & 1 & & & \\
\hline 427 & 8 & $0-10$ & 1 & 1 & 1 & 0 & 1 & & & \\
\hline 427 & 8 & $0-10$ & 1 & 2 & 1 & 0 & 1 & & & \\
\hline 427 & 8 & $0-10$ & 1 & 1 & 1 & 0 & 1 & & & \\
\hline 427 & 8 & $0-10$ & 1 & 1 & 1 & 0 & 1 & & & \\
\hline 427 & 8 & $0-10$ & 1 & 1 & 1 & 38 & 1 & & & \\
\hline 427 & 8 & $0-10$ & 1 & 2 & 1 & 0 & 1 & & & \\
\hline 427 & 8 & $0-10$ & 1 & 1 & 1 & 0 & 1 & & & \\
\hline 427 & 8 & $0-10$ & 1 & 1 & 1 & 0 & 1 & & & \\
\hline 427 & 8 & $0-10$ & 1 & 1 & 1 & 0 & 1 & & & \\
\hline 427 & 8 & $0-10$ & 1 & 1 & 1 & 0 & 1 & & & \\
\hline 427 & 8 & $0-10$ & 1 & 1 & 1 & 38 & 1 & & & \\
\hline 427 & 8 & $10-20$ & 6 & 3 & 1 & 0 & 1 & & & \\
\hline 427 & 8 & 10-20 & 1 & 2 & 1 & 0 & 1 & & & \\
\hline 427 & 8 & $10-20$ & 1 & 1 & 1 & 0 & 1 & & & \\
\hline 427 & 8 & $10-20$ & 1 & 1 & 1 & 0 & 1 & & & \\
\hline 427 & 8 & $10-20$ & 1 & 1 & 1 & 0 & 4 & & & \\
\hline 427 & 8 & $10-20$ & 1 & 2 & 1 & 38 & 1 & & & \\
\hline 427 & 8 & $10-20$ & 7 & - & $\ldots$ & . & - & & $\mathrm{X}$ & \\
\hline 427 & 8 & $10-20$ & - & - & - & - & - & Limestone & & \\
\hline 427 & 9 & $0-10$ & 1 & 2 & 1 & 88 & 1 & & & \\
\hline 427 & 9 & $0-10$ & 1 & 3 & 1 & 0 & 1 & & & \\
\hline 427 & 9 & $0-10$ & ـ & _. & - & - & _- & Limestone & & \\
\hline 427 & 9 & $40-50$ & 1 & 1 & 1 & 0 & 1 & & & \\
\hline
\end{tabular}


Table B-1. continued...

\begin{tabular}{|c|c|c|c|c|c|c|c|c|c|c|}
\hline $\begin{array}{r}\text { Site } \\
\text { 41TG } \\
\end{array}$ & $\begin{array}{l}\text { Shovel } \\
\text { Test \# }\end{array}$ & $\begin{array}{c}\text { Depth } \\
\text { cm }\end{array}$ & \begin{tabular}{|c|} 
Artifact \\
Type
\end{tabular} & $\begin{array}{l}\text { Max. } \\
\text { Length }\end{array}$ & $\begin{array}{c}\text { Max. } \\
\text { Thickness }\end{array}$ & $\begin{array}{c}\text { Cortex } \\
\%\end{array}$ & \begin{tabular}{|c|}
$\begin{array}{c}\text { Material } \\
\text { Color }\end{array}$ \\
\end{tabular} & $\begin{array}{c}\text { Fire Cracked } \\
\text { Rock }\end{array}$ & $\begin{array}{c}\begin{array}{c}\text { Mussel } \\
\text { Shell }\end{array} \\
\end{array}$ & $\begin{array}{c}\text { Other / } \\
\text { Remarks }\end{array}$ \\
\hline 427 & 10 & $0-10$ & - & - & - & - & - & Limestone & & \\
\hline 429 & 2 & $0-10$ & 6 & 4 & 1 & 12 & 1 & & & \\
\hline 429 & 2 & $0-10$ & 1 & 4 & 1 & 12 & 1 & & & \\
\hline 429 & 2 & $20-30$ & - & - & - & - & - & Limestone & & \\
\hline 429 & 2 & $40-50$ & 1 & 2 & 1 & 0 & 1 & & & \\
\hline 429 & 2 & $50-60$ & 1 & 2 & 1 & 12 & 1 & & & \\
\hline 429 & 2 & 50-60 & 1 & 2 & 1 & 100 & 1 & & & \\
\hline 429 & 2 & $60-70$ & 1 & 1 & 1 & 12 & 9 & & & \\
\hline 429 & 3 & $0-10$ & 1 & 2 & 1 & 0 & 1 & & & \\
\hline 429 & 3 & $0-10$ & 1 & 1 & 1 & 0 & 1 & & & \\
\hline 429 & 5 & $0-10$ & - & - & 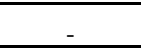 & 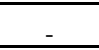 & - & & & Charcoal \\
\hline 429 & 5 & $10-20$ & 6 & 4 & 1 & 62 & 1 & & & Spokeshave? \\
\hline 429 & 5 & $10-20$ & 1 & 5 & 2 & 12 & 1 & & & \\
\hline 429 & 6 & $10-20$ & - & - & - & - & - & & & Charcoal \\
\hline 429 & 6 & $10-20$ & 6 & 3 & 1 & 0 & 1 & & & Burin \\
\hline 429 & 6 & 10-20 & 1 & 1 & 1 & 12 & 1 & & & \\
\hline 429 & 6 & $10-20$ & 1 & 2 & 1 & 0 & 1 & & & \\
\hline 429 & 7 & $30-40$ & 1 & 5 & 1 & 0 & 1 & & & \\
\hline 429 & 7 & $40-50$ & 1 & 2 & 1 & 88 & 1 & & & \\
\hline 429 & 7 & $50-60$ & 7 & - & - & - & - & & $\mathrm{X}$ & \\
\hline 430 & 2 & $10-20$ & 1 & 3 & 1 & 12 & 1 & & & \\
\hline 431 & 1 & - & - & - & - & - & - & Limestone & & \\
\hline 432 & 1 & $0-10$ & 1 & 2 & 1 & 0 & 1 & & & \\
\hline 433 & 1 & $40-50$ & 1 & 3 & 1 & 0 & 1 & & & Blade \\
\hline 433 & 2 & $20-30$ & - & - & - & - & - & Limestone & & \\
\hline 436 & 1 & $0-10$ & 1 & 4 & 1 & 0 & 1 & & & \\
\hline 436 & 1 & $0-10$ & 1 & 2 & 1 & 12 & 1 & & & \\
\hline 436 & 1 & $10-20$ & 1 & 4 & 1 & 0 & 1 & Sandstone & & \\
\hline 436 & 2 & $20-30$ & 1 & 3 & 1 & 0 & 1 & & & \\
\hline 436 & 2 & $20-30$ & 1 & 2 & 1 & 0 & 1 & & & \\
\hline 437 & 1 & $0-10$ & 1 & 2 & 1 & 0 & 1 & Chert & & \\
\hline 437 & 1 & $10-20$ & 1 & 5 & 2 & 38 & 1 & & & \\
\hline 437 & 1 & $30-40$ & 1 & 2 & 1 & 0 & 1 & & & Flaked edge \\
\hline 437 & 1 & $30-40$ & 1 & 2 & 1 & 0 & 1 & & & \\
\hline 437 & 2 & $0-10$ & 1 & 4 & 1 & 0 & 1 & & & \\
\hline 437 & 2 & $10-20$ & 6 & 3 & 1 & 12 & 1 & & & \\
\hline 437 & 2 & $10-20$ & 1 & 2 & 1 & 88 & 1 & & & \\
\hline 437 & 2 & $30-40$ & 1 & 3 & 1 & 0 & 1 & & & \\
\hline 437 & 2 & $40-50$ & 3 & 8 & 2 & 88 & 1 & & & \\
\hline 437 & 2 & $40-50$ & 1 & 3 & 1 & 0 & 1 & & & \\
\hline 437 & 2 & $40-50$ & 1 & 5 & 1 & 12 & 1 & & & \\
\hline 437 & 3 & $10-20$ & 4 & 6 & 2 & 0 & 1 & & & Crudely worked \\
\hline 437 & 3 & $10-20$ & 1 & 2 & 1 & 0 & 1 & & & \\
\hline 437 & 3 & $10-20$ & 1 & 2 & 1 & 12 & 1 & & & \\
\hline 437 & 3 & $20-30$ & 1 & 2 & 1 & 0 & 1 & Limestone & & \\
\hline 437 & 3 & $30-40$ & - & 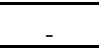 & - & - & - & & & Flaked clear glass \\
\hline 437 & 3 & $30-40$ & 1 & 2 & 1 & 0 & 1 & & & \\
\hline
\end{tabular}


Table B-1. continued...

\begin{tabular}{|c|c|c|c|c|c|c|c|c|c|c|}
\hline $\begin{array}{r}\text { Site } \\
\text { 41TG } \\
\end{array}$ & $\begin{array}{l}\text { Shovel } \\
\text { Test \# }\end{array}$ & $\begin{array}{c}\text { Depth } \\
\text { cm }\end{array}$ & $\begin{array}{c}\text { Artifact } \\
\text { Type }\end{array}$ & $\begin{array}{c}\text { Max. } \\
\text { Length }\end{array}$ & $\begin{array}{c}\text { Max. } \\
\text { Thickness } \\
\end{array}$ & $\begin{array}{c}\text { Cortex } \\
\%\end{array}$ & $\begin{array}{c}\text { Material } \\
\text { Color }\end{array}$ & $\begin{array}{c}\text { Fire Cracked } \\
\text { Rock }\end{array}$ & $\begin{array}{c}\text { Mussel } \\
\text { Shell }\end{array}$ & $\begin{array}{c}\text { Other / } \\
\text { Remarks }\end{array}$ \\
\hline 437 & 4 & $20-30$ & 1 & 2 & 1 & 0 & 1 & & & \\
\hline 437 & 4 & $20-30$ & 1 & 2 & 1 & 0 & 1 & & & \\
\hline 437 & 4 & $30-40$ & 1 & 2 & 1 & 0 & 1 & & & \\
\hline 437 & 6 & $0-10$ & 1 & 3 & 1 & 0 & 1 & & & \\
\hline 437 & 6 & $0-10$ & 1 & 2 & 1 & 0 & 1 & & & \\
\hline 437 & 6 & $0-10$ & 1 & 2 & 1 & 0 & 1 & & & \\
\hline 437 & 6 & $0-10$ & 1 & 1 & 1 & 0 & 1 & & & \\
\hline 437 & 6 & $10-20$ & 1 & 2 & 1 & 0 & 1 & & & \\
\hline 438 & 1 & $20-30$ & 6 & 4 & 1 & 0 & 1 & & & \\
\hline 438 & 1 & $30-40$ & 1 & 2 & 1 & 0 & 1 & & & \\
\hline 438 & 1 & $30-40$ & 1 & 2 & 1 & 0 & 1 & & & \\
\hline 438 & 1 & $30-40$ & 1 & 2 & 1 & 0 & 1 & & & \\
\hline 438 & 1 & $30-40$ & 1 & 1 & 1 & 0 & 1 & & & \\
\hline 438 & 1 & $30-40$ & 1 & 2 & 1 & 12 & 1 & & & \\
\hline 438 & 1 & $30-40$ & 1 & 1 & 1 & 0 & 1 & & & \\
\hline 438 & 1 & $30-40$ & 1 & 1 & 1 & 0 & 1 & & & \\
\hline 438 & 1 & $50-60$ & 1 & 2 & 1 & 0 & 1 & & & \\
\hline 438 & 1 & $60-70$ & 1 & 4 & 1 & 12 & 1 & & & \\
\hline 438 & 3 & $0-10$ & 1 & 2 & 1 & 0 & 1 & & & \\
\hline 438 & 4 & $10-20$ & 1 & 3 & 1 & 0 & 3 & & & \\
\hline 438 & 4 & $10-20$ & 1 & 2 & 1 & 12 & 1 & & & \\
\hline 438 & 4 & $10-20$ & 1 & 1 & 1 & 0 & 1 & & & \\
\hline 438 & 4 & $10-20$ & 3 & 8 & 4 & 75 & 1 & & & \\
\hline 438 & 4 & $30-40$ & 1 & 2 & 1 & 0 & 1 & & & \\
\hline 438 & 4 & $50-60$ & 1 & 2 & 1 & 0 & 1 & & & \\
\hline 438 & 5 & $60-70$ & 3 & 8 & 3 & 75 & 1 & & & \\
\hline 438 & 6 & $20-30$ & 1 & 2 & 1 & 0 & 1 & & & \\
\hline 438 & 6 & $30-40$ & 1 & 2 & 1 & 0 & 1 & & & \\
\hline 438 & 6 & $30-40$ & 1 & 2 & 1 & 0 & 1 & & & \\
\hline 438 & 6 & $30-40$ & 1 & 2 & 1 & 0 & 1 & & & \\
\hline 438 & 6 & $30-40$ & 1 & 1 & 1 & 0 & 1 & & & \\
\hline 439 & 3 & $0-10$ & 1 & 2 & 1 & 0 & 1 & & & \\
\hline 439 & 3 & $20-30$ & 1 & 3 & 1 & 12 & 1 & & & \\
\hline 441 & 1 & $0-10$ & 1 & 2 & 1 & 0 & 1 & & & \\
\hline 441 & 1 & $0-10$ & 1 & 2 & 1 & 88 & 1 & & & \\
\hline 441 & 1 & $0-10$ & 1 & 2 & 1 & 12 & 1 & & & \\
\hline 441 & 1 & $0-10$ & 1 & 3 & 1 & 88 & 1 & & & \\
\hline 441 & 1 & $20-30$ & 1 & 2 & 1 & 12 & 1 & & & \\
\hline 441 & 2 & $10-20$ & 1 & 2 & 1 & 0 & 1 & & & \\
\hline 441 & & $30-40$ & - & - & - & - & - & Limestone & & \\
\hline 444 & 1 & $0-10$ & 1 & 3 & 1 & 12 & 1 & & & \\
\hline 447 & 1 & $0-10$ & 4 & 6 & 2 & 12 & 1 & & & Heat-treated preform \\
\hline 447 & 1 & $10-20$ & 1 & 3 & 1 & 0 & 1 & & & \\
\hline 447 & 1 & $10-20$ & 1 & 2 & 1 & 0 & 1 & & & \\
\hline 447 & 2 & $10-20$ & 1 & 2 & 1 & 0 & - & Sandstone & & \\
\hline 447 & 2 & $30-40$ & 1 & 3 & 1 & 0 & 1 & & & \\
\hline 447 & 3 & $0-10$ & 1 & 3 & 1 & 88 & 1 & & & \\
\hline
\end{tabular}


Table B-1. continued...

\begin{tabular}{|c|c|c|c|c|c|c|c|c|c|c|}
\hline $\begin{array}{r}\text { Site } \\
\text { 41TG } \\
\end{array}$ & $\begin{array}{l}\text { Shovel } \\
\text { Test \# }\end{array}$ & $\begin{array}{c}\text { Depth } \\
\text { cm }\end{array}$ & \begin{tabular}{|c}
$\begin{array}{c}\text { Artifact } \\
\text { Tvpe }\end{array}$ \\
\end{tabular} & $\begin{array}{c}\text { Max. } \\
\text { Length }\end{array}$ & $\begin{array}{c}\text { Max. } \\
\text { Thickness }\end{array}$ & $\begin{array}{c}\text { Cortex } \\
\% \\
\end{array}$ & \begin{tabular}{|c|} 
Material \\
Color \\
\end{tabular} & $\begin{array}{c}\text { Fire Cracked } \\
\text { Rock }\end{array}$ & $\begin{array}{c}\text { Mussel } \\
\text { Shell }\end{array}$ & $\begin{array}{c}\text { Other / } \\
\text { Remarks }\end{array}$ \\
\hline 447 & 3 & $0-10$ & 1 & 4 & 1 & 0 & 1 & & & \\
\hline 447 & 3 & $0-10$ & 1 & 3 & 1 & 12 & 1 & & & \\
\hline 447 & 4 & $0-10$ & 1 & 2 & 1 & 0 & - & Chert & & \\
\hline 447 & 4 & $0-10$ & 1 & 2 & 1 & 0 & 1 & & & \\
\hline 447 & 4 & $0-10$ & 1 & 2 & 1 & 0 & 1 & & & \\
\hline 447 & 4 & $10-20$ & - & - & - & - & - & Sandstone & & \\
\hline 447 & 5 & $0-10$ & 6 & 4 & 1 & 0 & 1 & Chert \& Limestone & & \\
\hline 447 & 5 & $0-10$ & 6 & 5 & 1 & 0 & 1 & & & \\
\hline 447 & 5 & $0-10$ & 1 & 3 & 1 & 0 & 1 & & & \\
\hline 447 & 5 & $0-10$ & 1 & 2 & 1 & 12 & 1 & & & \\
\hline 447 & 5 & $0-10$ & 1 & 2 & 1 & 0 & 1 & & & \\
\hline 447 & 5 & $0-10$ & 1 & 2 & 1 & 0 & 1 & & & \\
\hline 447 & 5 & $0-10$ & 1 & 2 & 1 & 0 & 1 & & & \\
\hline 447 & 5 & $0-10$ & 1 & 2 & 1 & 0 & 1 & & & \\
\hline 447 & 5 & $0-10$ & 1 & 2 & 1 & 0 & 1 & & & \\
\hline 447 & 5 & $0-10$ & 1 & 3 & 1 & 0 & 1 & & & \\
\hline 447 & 5 & $10-20$ & 1 & 2 & 1 & 0 & 1 & Limestone & & \\
\hline 447 & 5 & $10-20$ & 1 & 2 & 1 & 0 & 1 & & & \\
\hline 447 & 5 & $10-20$ & 1 & 2 & 1 & 0 & 1 & & & \\
\hline 447 & 5 & $10-20$ & 1 & 2 & 1 & 0 & 1 & & & \\
\hline 447 & 5 & $10-20$ & 1 & 2 & 1 & 0 & 1 & & & \\
\hline 447 & 5 & $10-20$ & 6 & 4 & 1 & 12 & 1 & & & \\
\hline 447 & 5 & $10-20$ & 1 & 3 & 1 & 12 & 1 & & & \\
\hline 447 & 5 & $10-20$ & 1 & 3 & 1 & 0 & 1 & Limestone & & \\
\hline 447 & 5 & $10-20$ & 1 & 2 & 1 & 12 & 1 & & & \\
\hline 447 & 5 & $20-30$ & 1 & 1 & 1 & 0 & 1 & & & \\
\hline 447 & 5 & $20-30$ & 1 & 2 & 1 & 0 & 1 & & & \\
\hline 447 & 5 & 20-30 & 1 & 2 & 1 & 0 & 1 & & & \\
\hline 447 & 5 & $20-30$ & 1 & 2 & 1 & 0 & 1 & & & \\
\hline 447 & 5 & $20-30$ & 1 & 2 & 1 & 0 & 1 & & & \\
\hline 447 & 5 & $20-30$ & 1 & 3 & 1 & 0 & 1 & & & \\
\hline 447 & 5 & $20-30$ & 1 & 3 & 1 & 88 & 1 & & & \\
\hline 447 & 5 & $20-30$ & 1 & 3 & 1 & 100 & 1 & & & \\
\hline 447 & 5 & $20-30$ & 1 & 4 & 1 & 0 & 3 & & & \\
\hline 447 & 5 & $30-40$ & 6 & 5 & 2 & 0 & 1 & Limestone & & \\
\hline 447 & 5 & $30-40$ & 1 & 2 & 1 & 0 & 1 & & & \\
\hline 447 & 5 & $30-40$ & 6 & 5 & 2 & 0 & 1 & & & \\
\hline 447 & 5 & $40-50$ & 1 & 5 & 2 & 12 & 1 & Chert & & \\
\hline 447 & 5 & $40-50$ & 6 & 3 & 1 & 12 & 1 & & & \\
\hline 447 & 5 & $40-50$ & 1 & 2 & 1 & 88 & 1 & & & \\
\hline 447 & 5 & $40-50$ & 1 & 2 & 1 & 0 & 1 & & & \\
\hline 447 & 5 & $40-50$ & 1 & 2 & 1 & 0 & 1 & & & \\
\hline 447 & 5 & $40-50$ & 1 & 2 & 1 & 0 & 1 & & & \\
\hline 447 & 5 & $40-50$ & 1 & 2 & 1 & 0 & 1 & & & \\
\hline 447 & 5 & $40-50$ & 1 & 2 & 1 & 12 & 1 & & & \\
\hline 447 & 5 & $40-50$ & 1 & 2 & 1 & 62 & 1 & & & \\
\hline 447 & 5 & $40-50$ & 1 & 2 & 1 & 88 & 1 & & & \\
\hline
\end{tabular}


Table B-1. continued...

\begin{tabular}{|c|c|c|c|c|c|c|c|c|c|c|}
\hline $\begin{array}{r}\text { Site } \\
41 \mathrm{TG} \\
\end{array}$ & $\begin{array}{l}\text { Shovel } \\
\text { Test \# }\end{array}$ & $\begin{array}{c}\text { Depth } \\
\text { cm }\end{array}$ & $\begin{array}{c}\text { Artifact } \\
\text { Type }\end{array}$ & $\begin{array}{c}\text { Max. } \\
\text { Length }\end{array}$ & $\begin{array}{c}\text { Max. } \\
\text { Thickness } \\
\end{array}$ & $\begin{array}{c}\text { Cortex } \\
\% \\
\end{array}$ & \begin{tabular}{|c|} 
Material \\
Color \\
\end{tabular} & $\begin{array}{c}\text { Fire Cracked } \\
\text { Rock }\end{array}$ & $\begin{array}{c}\text { Mussel } \\
\text { Shell }\end{array}$ & $\begin{array}{c}\text { Other / } \\
\text { Remarks }\end{array}$ \\
\hline 447 & 5 & $50-60$ & 1 & 3 & 1 & 12 & 1 & Limestone & & \\
\hline 447 & 5 & $50-60$ & 1 & 3 & 1 & 12 & 1 & & & \\
\hline 447 & 5 & $50-60$ & 1 & 2 & 1 & 0 & 2 & & & \\
\hline 447 & 5 & $50-60$ & 1 & 2 & 1 & 12 & 1 & & & \\
\hline 447 & 6 & $0-10$ & - & - & - & - & - & Sandstone & & \\
\hline 447 & 6 & $0-10$ & - & - & - & - & - & & $\mathrm{X}$ & \\
\hline 447 & 6 & $0-10$ & 1 & 2 & 1 & 0 & 1 & & & \\
\hline 447 & 6 & $0-10$ & 1 & 2 & 1 & 0 & 1 & & & \\
\hline 447 & 6 & $0-10$ & 1 & 2 & 1 & 38 & 3 & & & \\
\hline 447 & 6 & $0-10$ & 1 & 2 & 1 & 0 & 3 & & & \\
\hline 447 & 6 & $0-10$ & 1 & 3 & 1 & 0 & 1 & & & \\
\hline 447 & 6 & $10-20$ & 1 & 2 & 1 & 0 & 1 & & & \\
\hline 447 & 6 & $20-30$ & 1 & 2 & 1 & 0 & 1 & & & \\
\hline 447 & 6 & $20-30$ & 1 & 2 & 1 & 0 & 1 & & & \\
\hline 447 & 6 & $20-30$ & 1 & 2 & 1 & 0 & 1 & & & \\
\hline 447 & 6 & $30-40$ & 1 & 2 & 1 & 0 & 2 & & & \\
\hline 447 & 6 & $30-40$ & 1 & 2 & 1 & 0 & 2 & & & \\
\hline 447 & 8 & $0-10$ & 1 & 3 & 1 & 0 & 1 & & & \\
\hline 447 & 9 & $0-10$ & 6 & 3 & 1 & 0 & 1 & & & \\
\hline 447 & 9 & $0-10$ & 1 & 2 & 1 & 0 & 1 & & & \\
\hline 447 & 10 & $0-10$ & 6 & 3 & 1 & 0 & 1 & & & \\
\hline 447 & 11 & $0-10$ & 1 & 4 & 2 & 0 & 1 & Limestone & & \\
\hline 447 & 11 & $0-10$ & 6 & 5 & 2 & 88 & 1 & & & \\
\hline 447 & 11 & $0-10$ & 1 & 2 & 1 & 38 & 1 & & & \\
\hline 447 & 11 & $0-10$ & 1 & 2 & 1 & 0 & 1 & & & \\
\hline 447 & 11 & $0-10$ & 1 & 2 & 1 & 0 & 1 & & & \\
\hline 447 & 13 & $0-10$ & 1 & 3 & 1 & 0 & 1 & Limestone & & \\
\hline 447 & 13 & $0-10$ & 1 & 3 & 1 & 0 & 1 & & & \\
\hline 447 & 13 & $0-10$ & 1 & 3 & 1 & 0 & 1 & & & \\
\hline 447 & 13 & $0-10$ & 1 & 3 & 1 & 88 & 1 & & & \\
\hline 447 & 13 & $0-10$ & 1 & 3 & 2 & 81 & 1 & & & \\
\hline 447 & 13 & $0-10$ & 1 & 2 & 1 & 0 & 1 & & & \\
\hline 447 & 13 & $0-10$ & 1 & 2 & 1 & 0 & 1 & & & \\
\hline 447 & 13 & $20-30$ & 1 & 3 & 1 & 0 & 3 & & & \\
\hline 447 & 13 & $20-30$ & 1 & 3 & 1 & 0 & 1 & & & \\
\hline 447 & 13 & $20-30$ & 1 & 2 & 1 & 0 & 1 & & & \\
\hline 447 & 13 & $20-30$ & 1 & 2 & 1 & 0 & 3 & & & \\
\hline 447 & 13 & $20-30$ & - & - & - & - & - & Limestone & & \\
\hline 447 & 13 & $30-40$ & 1 & 3 & 1 & 0 & 1 & & & \\
\hline 447 & 13 & $30-40$ & 1 & 3 & 1 & 0 & 1 & & & \\
\hline 447 & 13 & $30-40$ & 1 & 2 & 1 & 0 & 1 & & & \\
\hline 447 & 13 & $30-40$ & 1 & 3 & 1 & 0 & 1 & & & \\
\hline 447 & 13 & $30-40$ & 1 & 1 & 1 & 0 & 1 & & & \\
\hline 447 & 13 & $30-40$ & 1 & 2 & 1 & 0 & 1 & & & \\
\hline 447 & 13 & $30-40$ & 1 & 1 & 1 & 0 & 3 & & & \\
\hline 447 & 13 & $30-40$ & - & - & - & - & - & Limestone & & \\
\hline 447 & 13 & $40-50$ & 1 & 4 & 1 & 0 & 1 & & & \\
\hline
\end{tabular}


Table B-1. continued...

\begin{tabular}{|c|c|c|c|c|c|c|c|c|c|c|}
\hline $\begin{array}{r}\text { Site } \\
41 \mathrm{TG} \\
\end{array}$ & $\begin{array}{c}\text { Shovel } \\
\text { Test \# }\end{array}$ & $\begin{array}{c}\text { Depth } \\
\mathrm{cm}\end{array}$ & $\begin{array}{c}\text { Artifact } \\
\text { Type }\end{array}$ & $\begin{array}{l}\text { Max. } \\
\text { Length }\end{array}$ & $\begin{array}{c}\text { Max. } \\
\text { Thickness }\end{array}$ & $\begin{array}{c}\text { Cortex } \\
\% \\
\end{array}$ & $\begin{array}{c}\text { Material } \\
\text { Color }\end{array}$ & $\begin{array}{c}\text { Fire Cracked } \\
\text { Rock }\end{array}$ & $\begin{array}{c}\text { Mussel } \\
\text { Shell }\end{array}$ & $\begin{array}{c}\text { Other / } \\
\text { Remarks }\end{array}$ \\
\hline 447 & 13 & $50-60$ & 1 & 3 & 1 & 0 & 1 & & & \\
\hline 447 & 13 & $60-70$ & 1 & 2 & 1 & 12 & 1 & & & \\
\hline 447 & 13 & $60-70$ & 4 & 6 & 1 & 0 & 1 & & & Finely flaked knife \\
\hline 447 & Auger & $100-110$ & 1 & 2 & 1 & 0 & 1 & & & \\
\hline 447 & Auger & $120-140$ & - & - & - & - & - & Limestone & & \\
\hline 447 & Auger & $140-160$ & 1 & 2 & 1 & 0 & 1 & & & \\
\hline 447 & Auger & $20-40$ & 1 & 2 & 1 & 0 & 1 & & & \\
\hline 447 & Auger & $20-40$ & 1 & 2 & 1 & 0 & 1 & & & \\
\hline 447 & AUGER & $20-40$ & 1 & 2 & 1 & 12 & 1 & & & \\
\hline 449 & 1 & $0-10$ & 1 & 3 & 1 & 0 & 1 & & & \\
\hline 449 & 1 & $0-10$ & 1 & 3 & 1 & 0 & 1 & & & \\
\hline 449 & 1 & $0-10$ & 1 & 3 & 1 & 0 & 1 & & & \\
\hline 449 & 1 & $0-10$ & 1 & 2 & 1 & 0 & 1 & & & \\
\hline 449 & 1 & $0-10$ & 2 & 5 & 4 & 25 & 1 & & & \\
\hline 449 & 1 & $0-10$ & - & - & - & - & - & & & Purple bottle glass sherd \\
\hline 449 & 1 & $10-20$ & 1 & 6 & 2 & 88 & 1 & & & \\
\hline 449 & 2 & $0-10$ & 1 & 5 & 1 & 0 & 1 & & & Brown bottle glass \\
\hline 449 & 2 & $10-20$ & 1 & 3 & 1 & 12 & 1 & Sandstone & & \\
\hline 449 & 2 & $30-40$ & - & - & - & - & - & Limestone & & \\
\hline 449 & 2 & $30-40$ & 1 & 3 & 1 & 0 & 1 & & & \\
\hline 449 & 2 & $30-40$ & 1 & 1 & 1 & 0 & 1 & & & \\
\hline 449 & 2 & $30-40$ & 1 & 2 & 1 & 0 & 1 & & & \\
\hline 449 & 2 & $30-40$ & 1 & 2 & 1 & 0 & 1 & & & \\
\hline 449 & 2 & $30-40$ & 1 & 2 & 1 & 0 & 1 & & & \\
\hline 449 & 2 & $30-40$ & 1 & 2 & 1 & 0 & 1 & & & \\
\hline 449 & 2 & $30-40$ & 1 & 2 & 1 & 0 & 1 & & & \\
\hline 449 & 2 & $40-50$ & 1 & 2 & 1 & 62 & 1 & & & \\
\hline 449 & 2 & $50-60$ & 1 & 3 & 1 & 0 & 1 & & & \\
\hline 449 & 2 & $50-60$ & 1 & 4 & 1 & 0 & 1 & & & \\
\hline 449 & 2 & $60-70$ & 1 & 2 & 1 & 0 & 1 & & & \\
\hline 449 & 3 & $0-10$ & 1 & 5 & 2 & 12 & 1 & & & \\
\hline 449 & 3 & $0-10$ & 6 & 5 & 1 & 0 & 1 & & & \\
\hline 449 & 3 & $0-10$ & 1 & 2 & 1 & 0 & 1 & & & \\
\hline 449 & 3 & $0-10$ & 1 & 2 & 1 & 0 & 1 & & & \\
\hline 449 & 3 & $0-10$ & 1 & 2 & 1 & 0 & 1 & & & \\
\hline 449 & 3 & $20-30$ & 1 & 2 & 1 & 0 & 1 & & & \\
\hline 449 & 3 & $30-40$ & 1 & 2 & 1 & 0 & 1 & & & \\
\hline 449 & 3 & $40-50$ & 1 & 3 & 1 & 0 & 1 & & & \\
\hline 449 & Auger & $120-140$ & 1 & 2 & 1 & 0 & 1 & & & \\
\hline 449 & Auger & $120-140$ & - & - & - & - & - & Limestone & & \\
\hline 449 & AUGER & $20-40$ & - & - & - & - & - & Limestone & & \\
\hline 449 & AUGER & $20-40$ & - & - & - & - & - & Sandstone & & \\
\hline 449 & AUGER & $20-40$ & 1 & 2 & 1 & 0 & 1 & & & Burned \\
\hline 449 & $\overline{\text { AUGER }}$ & $20-40$ & 1 & 2 & 1 & 0 & 1 & & & Burned \\
\hline 449 & AUGER & $20-40$ & 1 & 2 & 1 & 0 & 1 & & & Burned \\
\hline 449 & AUGER & $80-100$ & - & - & - & - & - & Limestone & & \\
\hline 451 & 1 & $0-10$ & 6 & 6 & 2 & 38 & 1 & & & \\
\hline
\end{tabular}


Table B-1. continued...

\begin{tabular}{|c|c|c|c|c|c|c|c|c|c|c|}
\hline $\begin{array}{r}\text { Site } \\
41 \mathrm{TG} \\
\end{array}$ & $\begin{array}{l}\text { Shovel } \\
\text { Test \# }\end{array}$ & $\begin{array}{c}\text { Depth } \\
\mathrm{cm}\end{array}$ & $\begin{array}{c}\text { Artifact } \\
\text { Type }\end{array}$ & $\begin{array}{l}\text { Max. } \\
\text { Length }\end{array}$ & $\begin{array}{c}\text { Max. } \\
\text { Thickness } \\
\end{array}$ & $\begin{array}{c}\text { Cortex } \\
\%\end{array}$ & \begin{tabular}{|c|} 
Material \\
Color \\
\end{tabular} & $\begin{array}{c}\text { Fire Cracked } \\
\text { Rock }\end{array}$ & $\begin{array}{c}\text { Mussel } \\
\text { Shell }\end{array}$ & $\begin{array}{c}\text { Other / } \\
\text { Remarks }\end{array}$ \\
\hline 451 & 6 & $0-10$ & 1 & 2 & 1 & 0 & 1 & & & \\
\hline 453 & 1 & $10-20$ & 1 & 2 & 1 & 0 & 1 & & & \\
\hline 453 & 1 & $10-20$ & 1 & 2 & 1 & 0 & 1 & & & \\
\hline 453 & 1 & $10-20$ & 1 & 2 & 1 & 0 & 1 & & & \\
\hline 453 & 1 & $10-20$ & 1 & 2 & 1 & 0 & 3 & & & \\
\hline 453 & 2 & $30-40$ & 1 & 2 & 1 & 0 & 1 & & & \\
\hline 453 & 2 & $30-40$ & 1 & 2 & 1 & 0 & 1 & & & \\
\hline 453 & 3 & $0-10$ & 6 & 2 & 1 & 0 & 1 & & & \\
\hline 454 & 4 & $60-70$ & 1 & 3 & 1 & 0 & 1 & & $\mathrm{X}$ & Charcoal \\
\hline 455 & 1 & $0-10$ & - & - & - & - & - & & & 5 Rusted Metal Frags \\
\hline 455 & 1 & $20-30$ & 1 & 3 & 1 & 12 & 1 & & & \\
\hline 455 & 1 & $20-30$ & 1 & 2 & 1 & 0 & 1 & & & \\
\hline 455 & 1 & $20-30$ & 1 & 2 & 1 & 0 & 1 & & & \\
\hline 455 & 5 & $20-30$ & 1 & 1 & 1 & 12 & 1 & & & \\
\hline 455 & 5 & $20-30$ & 7 & - & - & - & - & & & 3 Metal fragments \\
\hline 456 & 11 & $0-10$ & 1 & 3 & 2 & 61 & 1 & & & \\
\hline 456 & 11 & $0-10$ & 2 & 6 & 3 & 12 & 1 & & & 5 Flake scars \\
\hline 457 & 2 & $50-60$ & 1 & 2 & 1 & 0 & 1 & & & \\
\hline 457 & 2 & $50-60$ & 1 & 2 & 1 & 0 & 1 & & & \\
\hline 457 & 2 & $50-60$ & - & - & - & - & - & Limestone & & \\
\hline 461 & 1 & $10-20$ & 1 & 1 & 1 & 0 & 1 & & & \\
\hline 461 & 1 & $10-20$ & 1 & 3 & 1 & 12 & 1 & & & \\
\hline 461 & 8 & $0-10$ & - & - & - & - & - & & & Fossilized Tooth Frag \\
\hline 461 & 8 & $0-10$ & 1 & 2 & 1 & 0 & 1 & & & \\
\hline 461 & AUGER1 & $0-10$ & 1 & 3 & 1 & 12 & 1 & & & \\
\hline 462 & 4 & $0-10$ & 1 & 2 & 1 & 0 & 1 & & & \\
\hline 462 & 4 & $10-20$ & - & - & - & - & - & Limestone & & Hearth feature \\
\hline 462 & 5 & $0-10$ & 1 & 4 & 1 & 12 & 1 & Limestone & & \\
\hline 462 & 7 & $0-10$ & 1 & 2 & 1 & 0 & 1 & & & \\
\hline 464 & 5 & $20-30$ & 1 & 2 & 1 & 0 & 1 & & & \\
\hline 464 & 7 & 44 & 1 & 2 & 1 & 0 & 1 & & & \\
\hline 464 & 7 & 44 & 5 & 8 & 2 & 0 & 1 & & & \\
\hline 464 & 7 & $0-10$ & - & - & - & - & - & Sandstone & & \\
\hline 464 & 7 & $30-40$ & 1 & 4 & 1 & 0 & 1 & & & \\
\hline 464 & 7 & $30-40$ & 1 & 4 & 1 & 0 & 1 & & & \\
\hline 464 & 7 & $50-60$ & 1 & 5 & 1 & 12 & 1 & & & Blade \\
\hline 464 & 7 & $50-60$ & 1 & 3 & 1 & 0 & 1 & & & Blade \\
\hline 464 & 7 & $50-60$ & 1 & 2 & 1 & 0 & 1 & & & \\
\hline 464 & 7 & $50-60$ & 1 & 2 & 1 & 12 & 1 & & & \\
\hline 464 & 7 & $50-60$ & 1 & 2 & 1 & 0 & 1 & & & \\
\hline 464 & 7 & $50-60$ & 1 & 2 & 1 & 12 & 1 & & & \\
\hline 464 & 7 & $50-60$ & 1 & 2 & 1 & 12 & 1 & & & \\
\hline 464 & 7 & $60-70$ & 1 & 2 & 1 & 0 & 1 & & & \\
\hline 464 & 7 & $60-70$ & 1 & 1 & 1 & 0 & 3 & & & \\
\hline 464 & 8 & $40-50$ & 1 & 2 & 1 & 12 & 1 & & & \\
\hline 464 & 8 & $40-50$ & 1 & 2 & 1 & 0 & 1 & & & \\
\hline 464 & 8 & $40-50$ & 1 & 1 & 1 & 0 & 1 & & & \\
\hline
\end{tabular}


Table B-1. continued...

\begin{tabular}{|c|c|c|c|c|c|c|c|c|c|c|}
\hline $\begin{array}{r}\text { Site } \\
\text { 41TG } \\
\end{array}$ & $\begin{array}{l}\text { Shovel } \\
\text { Test \# }\end{array}$ & $\begin{array}{c}\text { Depth } \\
\text { cm }\end{array}$ & \begin{tabular}{|c}
$\begin{array}{c}\text { Artifact } \\
\text { Tvpe }\end{array}$ \\
\end{tabular} & $\begin{array}{c}\text { Max. } \\
\text { Length }\end{array}$ & $\begin{array}{c}\text { Max. } \\
\text { Thickness }\end{array}$ & $\begin{array}{c}\text { Cortex } \\
\% \\
\end{array}$ & \begin{tabular}{|c} 
Material \\
Color
\end{tabular} & $\begin{array}{c}\begin{array}{c}\text { Fire Cracked } \\
\text { Rock }\end{array} \\
\end{array}$ & $\begin{array}{c}\text { Mussel } \\
\text { Shell } \\
\end{array}$ & $\begin{array}{c}\text { Other / } \\
\text { Remarks }\end{array}$ \\
\hline 464 & 8 & $50-60$ & 1 & 2 & 1 & 12 & 1 & & & \\
\hline 464 & 8 & $50-60$ & 1 & 2 & 1 & 0 & 1 & & & \\
\hline 464 & 8 & $50-60$ & 2 & 13 & 3 & 75 & 1 & & & \\
\hline 464 & 9 & $40-50$ & 2 & 7 & 4 & 25 & 1 & & & Burned \\
\hline 464 & 9 & $40-50$ & 1 & 2 & 1 & 0 & 1 & & & \\
\hline 464 & 9 & $40-50$ & 1 & 1 & 1 & 0 & 1 & & & \\
\hline 467 & 3 & $20-30$ & 1 & 2 & 1 & 0 & 1 & & & \\
\hline 467 & 3 & $20-30$ & 1 & 1 & 1 & 0 & 1 & & & \\
\hline 467 & 3 & $20-30$ & 4 & 8 & 3 & 25 & 1 & & & \\
\hline 467 & 3 & $30-40$ & 1 & 1 & 1 & 0 & 1 & & & \\
\hline 467 & 3 & $30-40$ & 1 & 2 & 1 & 0 & 1 & & & \\
\hline 467 & 4 & $0-10$ & 1 & 4 & 1 & 12 & 1 & & & \\
\hline 467 & 4 & $0-10$ & 1 & 1 & 1 & 0 & 1 & & & \\
\hline 467 & 4 & $0-10$ & 1 & 2 & 1 & 100 & 3 & & & \\
\hline 467 & 4 & $0-10$ & 1 & 1 & 1 & 0 & 1 & & & \\
\hline 467 & 4 & $0-10$ & 1 & 2 & 1 & 0 & 1 & & & \\
\hline 467 & 4 & $0-10$ & 1 & 2 & 1 & 0 & 1 & & & \\
\hline 467 & 4 & $0-10$ & 7 & - & - & - & - & & & Clear glass sherd \\
\hline 467 & 4 & $10-20$ & 1 & 2 & 1 & 0 & 1 & & & \\
\hline 467 & 5 & $0-10$ & 1 & 2 & 1 & 0 & 1 & & & \\
\hline 467 & 5 & $0-10$ & - & - & - & - & - & Limestone & & \\
\hline 467 & 6 & $0-10$ & 1 & 3 & 1 & 12 & 1 & & & \\
\hline 467 & 6 & $0-10$ & 1 & 1 & 1 & 0 & 1 & & & \\
\hline 467 & 6 & $0-10$ & 1 & 2 & 1 & 38 & 2 & & & \\
\hline 467 & 7 & $0-10$ & 1 & 3 & 2 & 38 & 1 & & & \\
\hline 467 & 10 & $0-10$ & 1 & 3 & 1 & 38 & 1 & & & \\
\hline 467 & 10 & $0-10$ & 1 & 1 & 1 & 0 & 1 & & & \\
\hline 467 & 10 & $0-10$ & 1 & 2 & 1 & 0 & 1 & & & \\
\hline 467 & 10 & $10-20$ & 6 & 6 & 1 & 88 & 4 & & & \\
\hline 468 & 3 & $10-20$ & - & - & - & - & - & & & Burned bone \\
\hline 471 & 2 & $10-20$ & 1 & 2 & 1 & 0 & 1 & & & \\
\hline 471 & 2 & $10-20$ & 1 & 2 & 1 & 0 & 1 & & & \\
\hline 471 & 3 & $10-20$ & 1 & 2 & 1 & 0 & 1 & & & \\
\hline 472 & 1 & $0-10$ & - & - & - & - & - & Sandstone & & \\
\hline 472 & 1 & $50-60$ & 1 & 2 & 1 & 0 & 1 & & & \\
\hline 472 & 1 & $50-60$ & 4 & 3 & 1 & 0 & 1 & & & \\
\hline 472 & 1 & $50-60$ & 1 & 3 & 1 & 0 & 1 & & & \\
\hline 472 & 1 & $50-60$ & 1 & 2 & 1 & 0 & 1 & & & \\
\hline 472 & 1 & $50-60$ & 1 & 2 & 1 & 0 & 1 & & & \\
\hline 472 & 1 & $50-60$ & 1 & 2 & 1 & 0 & 1 & & & \\
\hline 472 & 1 & $50-60$ & 1 & 2 & 1 & 0 & 1 & & & \\
\hline 472 & 1 & $50-60$ & 1 & 2 & 1 & 0 & 1 & & & \\
\hline 472 & 1 & $50-60$ & 1 & 3 & 1 & 0 & 1 & & & \\
\hline 472 & 1 & $50-60$ & 1 & 3 & 1 & 0 & 1 & & & \\
\hline 472 & 1 & $50-60$ & 1 & 1 & 1 & 0 & 1 & & & \\
\hline 472 & 1 & $50-60$ & 1 & 2 & 1 & 0 & 1 & & & \\
\hline 472 & 1 & $50-60$ & 1 & 2 & 1 & 0 & 1 & & & \\
\hline
\end{tabular}


Table B-1. continued...

\begin{tabular}{|c|c|c|c|c|c|c|c|c|c|c|}
\hline $\begin{array}{r}\text { Site } \\
\text { 41TG }\end{array}$ & $\begin{array}{l}\text { Shovel } \\
\text { Test \# }\end{array}$ & $\begin{array}{c}\text { Depth } \\
\text { cm }\end{array}$ & $\begin{array}{c}\text { Artifact } \\
\text { Type }\end{array}$ & $\begin{array}{l}\text { Max. } \\
\text { Length }\end{array}$ & $\begin{array}{c}\text { Max. } \\
\text { Thickness } \\
\end{array}$ & $\begin{array}{c}\text { Cortex } \\
\% \\
\end{array}$ & $\begin{array}{c}\text { Material } \\
\text { Color }\end{array}$ & $\begin{array}{c}\text { Fire Cracked } \\
\text { Rock }\end{array}$ & $\begin{array}{c}\text { Mussel } \\
\text { Shell }\end{array}$ & $\begin{array}{r}\text { Other / } \\
\text { Remarks }\end{array}$ \\
\hline 472 & 1 & $50-60$ & 1 & 4 & 1 & 0 & 1 & & & \\
\hline 472 & 1 & $50-60$ & 1 & 1 & 1 & 12 & 1 & & & \\
\hline 472 & 1 & $50-60$ & 1 & 2 & 1 & 12 & 1 & & & \\
\hline 472 & 1 & $50-60$ & 1 & 3 & 1 & 0 & 1 & & & \\
\hline 472 & 1 & $50-60$ & 1 & 3 & 1 & 0 & 1 & & & \\
\hline 472 & 1 & $50-60$ & 6 & 3 & 1 & 12 & 1 & & & \\
\hline 472 & 1 & $50-60$ & 1 & 3 & 1 & 0 & 1 & & & \\
\hline 472 & 1 & $50-60$ & 1 & 2 & 1 & 0 & 1 & & & \\
\hline 472 & 1 & $50-60$ & 1 & 2 & 1 & 0 & 1 & & & \\
\hline 472 & 1 & 50-60 & 1 & 2 & 1 & 0 & 1 & & & \\
\hline 472 & 1 & $50-60$ & 1 & 2 & 1 & 0 & 1 & & & \\
\hline 472 & 1 & $50-60$ & 1 & 2 & 1 & 0 & 1 & & & \\
\hline 472 & 1 & $50-60$ & 1 & 2 & 1 & 0 & 1 & & & \\
\hline 472 & 1 & $50-60$ & 1 & 2 & 1 & 0 & 1 & & & \\
\hline 472 & 1 & $50-60$ & 1 & 2 & 1 & 0 & 1 & & & \\
\hline 472 & 1 & $50-60$ & 1 & 2 & 1 & 0 & 1 & & & \\
\hline 472 & 1 & $50-60$ & 1 & 2 & 1 & 0 & 1 & & & \\
\hline 472 & 1 & $50-60$ & 1 & 2 & 1 & 0 & 1 & & & \\
\hline 472 & 1 & $50-60$ & 1 & 2 & 1 & 0 & 1 & & & \\
\hline 472 & 1 & $50-60$ & 1 & 2 & 1 & 0 & 1 & & & \\
\hline 472 & 1 & $50-60$ & 1 & 2 & 1 & 0 & 1 & & & \\
\hline 472 & 1 & $50-60$ & 1 & 2 & 1 & 0 & 1 & & & \\
\hline 472 & 1 & $50-60$ & 1 & 2 & 1 & 0 & 1 & & & \\
\hline 472 & 1 & $50-60$ & 1 & 2 & 1 & 0 & 1 & & & \\
\hline 472 & 1 & $50-60$ & 1 & 2 & 1 & 0 & 1 & & & \\
\hline 472 & 1 & $50-60$ & 1 & 2 & 1 & 0 & 1 & & & \\
\hline 472 & 1 & $50-60$ & 1 & 2 & 1 & 0 & 1 & & & \\
\hline 472 & 1 & $50-60$ & 1 & 2 & 1 & 0 & 1 & & & \\
\hline 472 & 1 & 50-60 & 1 & 2 & 1 & 0 & 1 & & & \\
\hline 472 & 1 & $50-60$ & 1 & 2 & 1 & 0 & 1 & & & \\
\hline 472 & 1 & $50-60$ & 1 & 2 & 1 & 0 & 1 & & & \\
\hline 472 & 1 & $50-60$ & 1 & 2 & 1 & 0 & 1 & & & \\
\hline 472 & 1 & $50-60$ & 1 & 2 & 1 & 0 & 1 & & & \\
\hline 472 & 1 & $50-60$ & 1 & 2 & 1 & 0 & 1 & & & \\
\hline 472 & 1 & $50-60$ & 1 & 2 & 1 & 0 & 2 & & & \\
\hline 472 & 1 & $60-70$ & 1 & 2 & 1 & 0 & 1 & & & \\
\hline 472 & 1 & $60-70$ & 1 & 2 & 1 & 0 & 1 & & & \\
\hline 472 & 1 & $60-70$ & 1 & 2 & 1 & 0 & 1 & & & \\
\hline 472 & 1 & $60-70$ & 1 & 2 & 1 & 0 & 1 & & & \\
\hline 472 & 1 & $60-70$ & 1 & 2 & 1 & 0 & 1 & & & \\
\hline 472 & 1 & $60-70$ & 1 & 2 & 1 & 0 & 1 & & & \\
\hline 472 & 2 & $0-10$ & 1 & 2 & 1 & 100 & 1 & & & \\
\hline 472 & 2 & $0-10$ & 1 & 3 & 1 & 0 & 1 & & & \\
\hline 472 & 2 & $0-10$ & 1 & 2 & 1 & 12 & 1 & & & \\
\hline 472 & 2 & $0-10$ & 1 & 2 & 1 & 0 & 1 & & & \\
\hline 472 & 2 & $0-10$ & 1 & 2 & 1 & 0 & 1 & & & \\
\hline 472 & 2 & $0-10$ & 1 & 1 & 1 & 0 & 1 & & & \\
\hline
\end{tabular}


Table B-1. continued...

\begin{tabular}{|c|c|c|c|c|c|c|c|c|c|c|}
\hline $\begin{array}{r}\text { Site } \\
\text { 41TG } \\
\end{array}$ & $\begin{array}{l}\text { Shovel } \\
\text { Test \# } \\
\end{array}$ & $\begin{array}{c}\text { Depth } \\
\text { cm }\end{array}$ & \begin{tabular}{|c}
$\begin{array}{c}\text { Artifact } \\
\text { Tvpe }\end{array}$ \\
\end{tabular} & $\begin{array}{c}\text { Max. } \\
\text { Length }\end{array}$ & $\begin{array}{c}\text { Max. } \\
\text { Thickness }\end{array}$ & $\begin{array}{c}\text { Cortex } \\
\% \\
\end{array}$ & \begin{tabular}{|c|} 
Material \\
Color \\
\end{tabular} & $\begin{array}{c}\text { Fire Cracked } \\
\text { Rock }\end{array}$ & $\begin{array}{c}\text { Mussel } \\
\text { Shell }\end{array}$ & $\begin{array}{c}\text { Other / } \\
\text { Remarks }\end{array}$ \\
\hline 472 & 2 & $0-10$ & 1 & 1 & 1 & 0 & 1 & & & \\
\hline 472 & 2 & $0-10$ & 7 & - & - & - & - & & & 3 Whiteware ceramics \\
\hline 472 & 2 & $10-20$ & - & - & - & - & - & Limestone & & \\
\hline 472 & 2 & $10-20$ & 1 & 2 & 1 & 0 & 1 & & & \\
\hline 472 & 2 & $10-20$ & 1 & 1 & 1 & 0 & 1 & & & \\
\hline 472 & 2 & $10-20$ & 1 & 2 & 1 & 0 & 1 & & & \\
\hline 472 & 2 & $10-20$ & 7 & - & - & - & - & & & Ochre? \\
\hline 472 & 2 & $20-30$ & 1 & 2 & 1 & 12 & 1 & & & \\
\hline 472 & 2 & $20-30$ & 7 & - & - & - & - & & & 2 Whiteware ceramics \\
\hline 472 & 3 & $0-10$ & 1 & 3 & 1 & 0 & 1 & Limestone \& Chert & & \\
\hline 472 & 3 & $10-20$ & 1 & 2 & 1 & 0 & 1 & & & \\
\hline 472 & 3 & $10-20$ & 1 & 2 & 1 & 0 & 1 & Limestone & & \\
\hline 472 & 3 & $10-20$ & 1 & 2 & 1 & 0 & 1 & & & \\
\hline 472 & 3 & $10-20$ & 1 & 1 & 1 & 0 & 1 & & & \\
\hline 472 & 3 & $10-20$ & 1 & 2 & 1 & 0 & 1 & & & \\
\hline 472 & 3 & $10-20$ & 1 & 1 & 1 & 0 & 1 & & & \\
\hline 472 & 3 & $10-20$ & 1 & 2 & 1 & 0 & 1 & & & \\
\hline 472 & 3 & $10-20$ & 1 & 2 & 1 & 12 & 1 & & & \\
\hline 472 & 3 & $10-20$ & 1 & 1 & 1 & 0 & 1 & & & \\
\hline 472 & 3 & $10-20$ & 1 & 2 & 1 & 0 & 1 & & & \\
\hline 472 & 3 & $10-20$ & 1 & 2 & 1 & 0 & 1 & & & \\
\hline 472 & 3 & $10-20$ & 1 & 2 & 1 & 0 & 1 & & & \\
\hline 472 & 3 & $10-20$ & 1 & 2 & 1 & 0 & 1 & & & \\
\hline 472 & 3 & $10-20$ & 1 & 2 & 1 & 0 & 1 & & & \\
\hline 472 & 3 & $10-20$ & 1 & 1 & 1 & 0 & 1 & & & \\
\hline 472 & 3 & $20-30$ & 1 & 2 & 1 & 0 & 1 & Limestone & & \\
\hline 472 & 3 & $20-30$ & 1 & 2 & 1 & 0 & 1 & & & \\
\hline 472 & 3 & $30-40$ & 1 & 2 & 1 & 0 & 1 & & & \\
\hline 472 & 3 & $30-40$ & 1 & 2 & 1 & 0 & 1 & & & \\
\hline 472 & 3 & $30-40$ & 1 & 2 & 1 & 0 & 1 & & & \\
\hline 472 & 3 & $50-60$ & 1 & 2 & 1 & 0 & 1 & & & \\
\hline 472 & 3 & $60-70$ & 1 & 3 & 1 & 88 & 1 & & & \\
\hline 472 & 3 & $60-70$ & 1 & 2 & 1 & 0 & 1 & & & \\
\hline 472 & 4 & $10-20$ & 1 & 3 & 1 & 38 & 1 & & & \\
\hline 472 & AUGER & $60-80$ & 1 & 2 & 1 & 0 & 1 & & & \\
\hline 474 & 2 & $20-30$ & 1 & 5 & 2 & 12 & 1 & & & \\
\hline 474 & 6 & $0-10$ & 1 & 2 & 1 & 0 & 1 & & & \\
\hline 474 & 6 & $0-10$ & 1 & 7 & 2 & 62 & 1 & & & \\
\hline 478 & 5 & $0-10$ & 3 & 5 & 4 & 88 & 1 & & & \\
\hline 478 & 5 & $0-10$ & 6 & 4 & 2 & 0 & 1 & & & \\
\hline 478 & 5 & $0-10$ & 1 & 3 & 1 & 38 & 1 & & & \\
\hline 478 & 5 & $0-10$ & 1 & 2 & 1 & 0 & 1 & & & \\
\hline 478 & 8 & $0-10$ & 6 & 4 & 1 & 62 & 1 & & & \\
\hline 478 & 10 & $0-10$ & 1 & 2 & 1 & 0 & 1 & & & \\
\hline 478 & 11 & $0-10$ & 1 & 4 & 1 & 12 & 1 & & & \\
\hline 479 & 2 & $0-10$ & 1 & 1 & 1 & 12 & 1 & & & \\
\hline 480 & 1 & $0-10$ & 1 & 3 & 1 & 88 & 1 & & & \\
\hline
\end{tabular}


Table B-1. continued...

\begin{tabular}{|c|c|c|c|c|c|c|c|c|c|c|}
\hline $\begin{array}{r}\text { Site } \\
\text { 41TG } \\
\end{array}$ & $\begin{array}{l}\text { Shovel } \\
\text { Test \# }\end{array}$ & $\begin{array}{c}\text { Depth } \\
\text { cm }\end{array}$ & $\begin{array}{c}\text { Artifact } \\
\text { Type }\end{array}$ & $\begin{array}{c}\text { Max. } \\
\text { Length }\end{array}$ & $\begin{array}{c}\text { Max. } \\
\text { Thickness }\end{array}$ & $\begin{array}{c}\text { Cortex } \\
\%\end{array}$ & $\begin{array}{c}\text { Material } \\
\text { Color }\end{array}$ & $\begin{array}{c}\text { Fire Cracked } \\
\text { Rock }\end{array}$ & $\begin{array}{c}\text { Mussel } \\
\text { Shell }\end{array}$ & $\begin{array}{c}\text { Other / } \\
\text { Remarks }\end{array}$ \\
\hline 480 & 2 & $30-40$ & 1 & 3 & 1 & 12 & 1 & & & \\
\hline 481 & 1 & $0-10$ & 1 & 4 & 1 & 0 & 1 & & & \\
\hline 481 & 2 & $0-10$ & 1 & 3 & 1 & 0 & 1 & & & \\
\hline 481 & 2 & $0-10$ & 1 & 2 & 1 & 0 & 1 & Chert & & \\
\hline 481 & 2 & $20-30$ & 1 & 4 & 2 & 12 & 1 & & & \\
\hline 481 & 2 & $30-40$ & 2 & 8 & 3 & 0 & 1 & & & 5 Flake scars \\
\hline 481 & 2 & $30-40$ & 1 & 6 & 2 & 0 & 1 & & & \\
\hline 481 & 3 & $10-20$ & 1 & 1 & 1 & 0 & 1 & & & \\
\hline 481 & 3 & $10-20$ & - & - & - & - & - & Chert & & \\
\hline 482 & 1 & $0-10$ & 1 & 2 & 1 & 0 & 1 & & & \\
\hline 482 & 1 & $10-20$ & 1 & 2 & 1 & 0 & 1 & & & \\
\hline 482 & 1 & $10-20$ & 1 & 2 & 1 & 0 & 1 & & & \\
\hline 485 & 1 & $10-20$ & 1 & 2 & 1 & 0 & 1 & & & \\
\hline 485 & 7 & $0-10$ & 6 & 5 & 1 & 0 & 1 & & & \\
\hline 485 & 9 & $0-10$ & 1 & 6 & 2 & 88 & 1 & & & \\
\hline 485 & 10 & $20-30$ & 1 & 2 & 1 & 0 & 1 & & & \\
\hline 485 & 11 & $0-10$ & 1 & 2 & 1 & 0 & 1 & & & \\
\hline 485 & 11 & $10-20$ & 1 & 2 & 1 & 38 & 1 & & & \\
\hline 485 & 12 & $0-10$ & 1 & 5 & 1 & 88 & 1 & & & \\
\hline 485 & 13 & $10-20$ & 6 & 3 & 1 & 0 & 1 & & & Burin \\
\hline 486 & 2 & $0-10$ & 1 & 3 & 1 & 38 & 1 & & & \\
\hline 486 & 2 & $30-40$ & 1 & 2 & 1 & 12 & 1 & & & \\
\hline 486 & 3 & $0-10$ & 1 & 6 & 2 & 0 & 1 & & & \\
\hline 486 & 4 & $0-10$ & 6 & 3 & 1 & 0 & 1 & & & \\
\hline 486 & 4 & $0-10$ & 1 & 2 & 1 & 0 & 1 & & & \\
\hline 486 & 4 & $0-10$ & 1 & 2 & 1 & 0 & 1 & & & \\
\hline 486 & 4 & $0-10$ & 1 & 2 & 1 & 62 & 1 & & & \\
\hline 486 & 4 & $0-10$ & 1 & 3 & 1 & 38 & 1 & & & \\
\hline 486 & 5 & $0-10$ & 1 & 2 & 1 & 0 & 1 & & & \\
\hline 486 & 5 & $0-10$ & 1 & 2 & 1 & 0 & 1 & & & \\
\hline 487 & 2 & $20-30$ & 3 & 6 & 4 & 75 & 1 & Limestone & & \\
\hline 487 & 3 & $10-20$ & 1 & 2 & 1 & 0 & 1 & & & \\
\hline 487 & 3 & $10-20$ & 1 & 3 & 1 & 0 & 4 & & & \\
\hline 487 & 4 & $20-30$ & 1 & 2 & 1 & 0 & 1 & & & \\
\hline 487 & 4 & $30-40$ & 1 & 3 & 1 & 12 & 1 & & & \\
\hline 487 & 4 & $30-40$ & 1 & 2 & 1 & 38 & 1 & & & \\
\hline 487 & 4 & $30-40$ & 1 & 2 & 1 & 0 & 2 & & & \\
\hline 487 & 4 & $30-40$ & 1 & 2 & 1 & 12 & 1 & & & \\
\hline 488 & 3 & $0-10$ & 1 & 5 & 1 & 12 & 1 & & & \\
\hline 488 & 6 & $0-10$ & 1 & 2 & 1 & 0 & 1 & & & \\
\hline 488 & 7 & $10-20$ & 2 & 5 & 3 & 38 & 1 & & & 3 Flake scars \\
\hline 489 & 1 & $0-10$ & 1 & 3 & 1 & 0 & 1 & & & \\
\hline 489 & 1 & $0-10$ & 1 & 3 & 1 & 0 & 1 & & & \\
\hline 489 & 2 & $0-10$ & 1 & 2 & 1 & 0 & 1 & & & \\
\hline 489 & 2 & $0-10$ & 1 & 2 & 1 & 0 & 4 & & & \\
\hline 489 & 3 & $0-10$ & 1 & 5 & 2 & 100 & 1 & Limestone \& Chert & $\mathrm{x}$ & \\
\hline 489 & 3 & $0-10$ & 1 & 2 & 1 & 0 & 1 & & & \\
\hline
\end{tabular}


Table B-1. continued...

\begin{tabular}{|c|c|c|c|c|c|c|c|c|c|c|}
\hline $\begin{array}{r}\text { Site } \\
\text { 41TG } \\
\end{array}$ & $\begin{array}{l}\text { Shovel } \\
\text { Test \# }\end{array}$ & $\begin{array}{c}\text { Depth } \\
\text { cm }\end{array}$ & \begin{tabular}{|c}
$\begin{array}{c}\text { Artifact } \\
\text { Tvpe }\end{array}$ \\
\end{tabular} & $\begin{array}{c}\text { Max. } \\
\text { Length }\end{array}$ & $\begin{array}{c}\text { Max. } \\
\text { Thickness }\end{array}$ & $\begin{array}{c}\text { Cortex } \\
\% \\
\end{array}$ & \begin{tabular}{|c|} 
Material \\
Color \\
\end{tabular} & $\begin{array}{c}\begin{array}{c}\text { Fire Cracked } \\
\text { Rock }\end{array} \\
\end{array}$ & $\begin{array}{c}\text { Mussel } \\
\text { Shell } \\
\end{array}$ & $\begin{array}{c}\text { Other / } \\
\text { Remarks }\end{array}$ \\
\hline 489 & 3 & $0-10$ & 1 & 3 & 1 & 12 & 1 & & & \\
\hline 489 & 3 & $0-10$ & 1 & 3 & 1 & 0 & 1 & & & \\
\hline 489 & 3 & $0-10$ & 1 & 3 & 1 & 0 & 1 & & & \\
\hline 489 & 3 & $0-10$ & 1 & 3 & 1 & 0 & 1 & & & \\
\hline 489 & 3 & $0-10$ & 1 & 2 & 1 & 0 & 1 & & & \\
\hline 489 & 4 & $0-10$ & 1 & 3 & 1 & 0 & 1 & & & \\
\hline 489 & 4 & $0-10$ & 1 & 3 & 1 & 0 & 1 & & & \\
\hline 489 & 5 & $0-10$ & 6 & 5 & 2 & 38 & 1 & & & \\
\hline 489 & 5 & $0-10$ & 1 & 3 & 1 & 88 & 1 & & & \\
\hline 489 & 5 & $0-10$ & 1 & 3 & 1 & 88 & 1 & & & \\
\hline 489 & 5 & $0-10$ & 1 & 1 & 1 & 0 & 1 & & & \\
\hline 489 & 5 & $0-10$ & 1 & 2 & 1 & 38 & 1 & & & \\
\hline 489 & 8 & $0-10$ & 1 & 3 & 1 & 0 & 2 & & & \\
\hline 489 & 9 & $0-10$ & 6 & 4 & 1 & 0 & 1 & & & \\
\hline 489 & 9 & $0-10$ & 1 & 2 & 1 & 12 & 2 & & & \\
\hline 489 & 9 & $0-10$ & 1 & 3 & 1 & 0 & 1 & & & \\
\hline 489 & 9 & $0-10$ & 1 & 2 & 1 & 0 & 2 & & & \\
\hline 489 & 9 & $0-10$ & 1 & 2 & 1 & 0 & 1 & & & \\
\hline 489 & 9 & $10-20$ & 1 & 3 & 1 & 0 & 1 & & & \\
\hline 489 & 9 & $10-20$ & 1 & 2 & 1 & 0 & 2 & & & \\
\hline 489 & 9 & $20-30$ & 1 & 3 & 1 & 100 & 1 & Limestone & & \\
\hline 489 & 9 & $20-30$ & 1 & 3 & 1 & 0 & 1 & & & \\
\hline 489 & 9 & $20-30$ & 1 & 2 & 1 & 0 & 1 & & & \\
\hline 489 & 9 & $20-30$ & 1 & 2 & 1 & 0 & 2 & & & \\
\hline 489 & 10 & $0-10$ & 1 & 2 & 1 & 0 & 1 & & & \\
\hline 489 & 10 & $10-20$ & 6 & 4 & 1 & 62 & 1 & & & \\
\hline 489 & 11 & $0-10$ & 1 & 2 & 1 & 0 & 3 & & & \\
\hline 489 & 13 & $0-10$ & - & - & - & - & - & Limestone & & \\
\hline 489 & 13 & $10-20$ & - & - & - & - & - & Limestone & & \\
\hline 489 & 14 & $0-10$ & 1 & 3 & 1 & 12 & 1 & & & \\
\hline 489 & 14 & $10-20$ & - & - & - & - & - & Limestone & & \\
\hline 489 & 15 & $20-30$ & 1 & 2 & 1 & 88 & 2 & & & \\
\hline 489 & 16 & $0-10$ & 1 & 2 & 1 & 0 & 1 & & & \\
\hline 489 & 16 & $0-10$ & 1 & 3 & 1 & 38 & 1 & & & \\
\hline 489 & 16 & $0-10$ & 1 & 2 & 1 & 0 & 1 & & & \\
\hline 489 & 16 & $0-10$ & 1 & 2 & 1 & 38 & 1 & & & \\
\hline 489 & 16 & $0-10$ & 1 & 2 & 1 & 0 & 1 & & & \\
\hline 489 & 16 & $0-10$ & 1 & 2 & 1 & 38 & 1 & & & \\
\hline 489 & 16 & $10-20$ & 1 & 3 & 1 & 38 & 1 & Limestone & & \\
\hline 489 & 16 & $10-20$ & 1 & 2 & 1 & 0 & 1 & & & \\
\hline 489 & 16 & $10-20$ & 1 & 1 & 1 & 0 & 1 & & & \\
\hline 489 & 16 & $10-20$ & 1 & 2 & 1 & 0 & 1 & & & \\
\hline 489 & 16 & $10-20$ & 1 & 2 & 1 & 38 & 2 & & & \\
\hline 489 & 16 & $10-20$ & 1 & 2 & 1 & 0 & 1 & & & \\
\hline 489 & 16 & $20-30$ & 1 & 2 & 1 & 38 & 1 & Sandstone & & \\
\hline 489 & 16 & $20-30$ & 1 & 2 & 1 & 62 & 1 & & & \\
\hline 489 & 16 & $20-30$ & 1 & 2 & 1 & 12 & 1 & & & \\
\hline
\end{tabular}


Table B-1. continued...

\begin{tabular}{|c|c|c|c|c|c|c|c|c|c|c|}
\hline $\begin{array}{r}\text { Site } \\
\text { 41TG }\end{array}$ & $\begin{array}{l}\text { Shovel } \\
\text { Test \# }\end{array}$ & $\begin{array}{c}\text { Depth } \\
\mathrm{cm}\end{array}$ & $\begin{array}{c}\text { Artifact } \\
\text { Tvpe }\end{array}$ & $\begin{array}{c}\text { Max. } \\
\text { Length }\end{array}$ & $\begin{array}{c}\text { Max. } \\
\text { Thickness } \\
\end{array}$ & $\begin{array}{c}\text { Cortex } \\
\% \\
\end{array}$ & $\begin{array}{c}\text { Material } \\
\text { Color }\end{array}$ & $\begin{array}{c}\text { Fire Cracked } \\
\text { Rock }\end{array}$ & $\begin{array}{c}\text { Mussel } \\
\text { Shell }\end{array}$ & $\begin{array}{c}\text { Other / } \\
\text { Remarks }\end{array}$ \\
\hline 491 & 1 & $30-40$ & 1 & 2 & 1 & 0 & 1 & & & \\
\hline 492 & 2 & $0-10$ & 1 & 5 & 2 & 88 & 1 & & & \\
\hline 492 & 2 & $40-50$ & 1 & 3 & 1 & 62 & 2 & & & \\
\hline 492 & 3 & $30-40$ & 1 & 2 & 1 & 0 & 1 & & & \\
\hline 495 & 3 & $0-10$ & 6 & 5 & 2 & 0 & 1 & & & \\
\hline 495 & 3 & $0-10$ & 1 & 3 & 1 & 12 & 1 & & & \\
\hline 496 & 1 & $10-20$ & 1 & 3 & 1 & 88 & 1 & & & \\
\hline 496 & 1 & $20-30$ & 1 & 3 & 1 & 12 & 1 & & & \\
\hline 496 & 2 & $10-20$ & 6 & 3 & 1 & 62 & 1 & & & \\
\hline 496 & 3 & $0-10$ & 1 & 7 & 3 & 88 & 1 & & & \\
\hline 496 & 3 & $0-10$ & 1 & 3 & 1 & 0 & 1 & & & \\
\hline 496 & 3 & $0-10$ & 1 & 3 & 2 & 88 & 1 & & & \\
\hline 496 & 3 & $0-10$ & 1 & 3 & 1 & 0 & 1 & & & \\
\hline 496 & 3 & $10-20$ & 1 & 2 & 1 & 88 & 1 & & & \\
\hline 496 & 3 & $10-20$ & 1 & 2 & 1 & 88 & 1 & & & \\
\hline 497 & 1 & $0-10$ & 1 & 3 & 2 & 88 & 1 & & & \\
\hline 497 & 5 & $0-10$ & 1 & 3 & 1 & 62 & 1 & & & \\
\hline 499 & 1 & $50-60$ & - & - & - & - & - & Sandstone & & Charcoal \\
\hline 500 & 2 & $0-10$ & 1 & 2 & 1 & 0 & 1 & & & \\
\hline 500 & 2 & $10-20$ & 1 & 2 & 1 & 0 & 1 & & & \\
\hline 500 & 2 & $20-30$ & 1 & 3 & 1 & 12 & 1 & & & \\
\hline 500 & 2 & $30-40$ & 1 & 3 & 1 & 0 & 1 & & & \\
\hline 500 & 2 & $30-40$ & 1 & 3 & 1 & 38 & 1 & & & \\
\hline 500 & 2 & $30-40$ & 1 & 3 & 1 & 0 & 1 & & & \\
\hline 500 & 2 & $30-40$ & 1 & 1 & 1 & 0 & 1 & & & \\
\hline 500 & 2 & $30-40$ & 1 & 1 & 1 & 0 & 1 & & & \\
\hline 500 & 2 & $30-40$ & 1 & 2 & 1 & 0 & 3 & & & \\
\hline 500 & 2 & $30-40$ & 1 & 1 & 1 & 0 & 1 & & & \\
\hline 500 & 3 & $0-10$ & - & _ & - & - & - & & $\mathrm{X}$ & \\
\hline 500 & 3 & $10-20$ & 1 & 1 & 1 & 0 & 1 & & & \\
\hline 500 & 3 & $10-20$ & - & - & - & - & - & Limestone & & \\
\hline 500 & 3 & $10-20$ & 1 & 6 & 2 & 88 & 1 & & & \\
\hline 500 & 3 & $40-50$ & - & - & - & - & - & Sandstone & & \\
\hline 500 & 5 & $10-20$ & 6 & 6 & 1 & 12 & 1 & & & Finely flaked \\
\hline 500 & 5 & $10-20$ & 1 & 3 & 1 & 0 & 1 & & & \\
\hline 500 & 5 & $10-20$ & 1 & 3 & 1 & 0 & 1 & & & \\
\hline 500 & 5 & $10-20$ & 1 & 1 & 1 & 12 & 1 & & & \\
\hline 500 & 5 & $10-20$ & 1 & 2 & 1 & 12 & 1 & & & \\
\hline 500 & 5 & $10-20$ & 1 & 2 & 1 & 12 & 1 & & & \\
\hline 500 & 5 & $10-20$ & 1 & 2 & 1 & 0 & 1 & & & \\
\hline 500 & 5 & $20-30$ & 1 & 2 & 1 & 0 & 1 & & & \\
\hline 500 & 5 & $20-30$ & 1 & 2 & 1 & 0 & 1 & & & \\
\hline 500 & 5 & $40-50$ & 1 & 2 & 1 & 0 & 1 & & & \\
\hline 500 & 6 & $0-10$ & 1 & 3 & 1 & 12 & 2 & & & \\
\hline 500 & 6 & $10-20$ & 1 & 2 & 1 & 0 & 1 & & & \\
\hline 500 & AUGER1 & $0-20$ & 1 & 5 & 1 & 12 & 1 & & & \\
\hline 504 & 1 & $20-30$ & 1 & 5 & 2 & 38 & 1 & Limestone & & \\
\hline
\end{tabular}


Table B-1. continued...

\begin{tabular}{|c|c|c|c|c|c|c|c|c|c|c|}
\hline $\begin{array}{r}\text { Site } \\
\text { 41TG }\end{array}$ & $\begin{array}{r}\text { Shovel } \\
\text { Test \# }\end{array}$ & $\begin{array}{c}\text { Depth } \\
\mathrm{cm}\end{array}$ & $\begin{array}{c}\text { Artifact } \\
\text { Type }\end{array}$ & $\begin{array}{c}\text { Max. } \\
\text { Length }\end{array}$ & $\begin{array}{c}\text { Max. } \\
\text { Thickness }\end{array}$ & $\begin{array}{c}\text { Cortex } \\
\% \\
\end{array}$ & \begin{tabular}{|c} 
Material \\
Color \\
\end{tabular} & $\begin{array}{c}\text { Fire Cracked } \\
\text { Rock }\end{array}$ & $\begin{array}{c}\text { Mussel } \\
\text { Shell }\end{array}$ & $\begin{array}{c}\text { Other / } \\
\text { Remarks }\end{array}$ \\
\hline 504 & 1 & $20-30$ & 1 & 5 & 3 & 12 & 1 & & & \\
\hline 504 & 1 & $20-30$ & 1 & 4 & 1 & 0 & 1 & & & \\
\hline 504 & 1 & $20-30$ & 1 & 3 & 1 & 0 & 1 & & & \\
\hline 504 & 1 & $20-30$ & 1 & 3 & 1 & 0 & 1 & & & \\
\hline 504 & 1 & $20-30$ & 1 & 2 & 1 & 0 & 1 & & & \\
\hline 504 & 1 & $20-30$ & 1 & 2 & 1 & 0 & 1 & & & \\
\hline 504 & 1 & $20-30$ & 1 & 2 & 1 & 0 & 1 & & & \\
\hline 504 & 1 & $20-30$ & 1 & 2 & 1 & 0 & 1 & & & \\
\hline 504 & 1 & $20-30$ & 1 & 2 & 1 & 0 & 1 & & & \\
\hline 504 & 1 & $20-30$ & 1 & 2 & 1 & 0 & 1 & & & \\
\hline 504 & 1 & $20-30$ & 1 & 2 & 1 & 0 & 1 & & & \\
\hline 504 & 1 & $20-30$ & 1 & 2 & 1 & 0 & 1 & & & \\
\hline 504 & 1 & $20-30$ & 1 & 2 & 1 & 0 & 1 & & & \\
\hline 504 & 1 & $20-30$ & 1 & 2 & 1 & 0 & 1 & & & \\
\hline 504 & 1 & $20-30$ & 1 & 2 & 1 & 0 & 1 & & & \\
\hline 504 & 1 & $20-30$ & 1 & 2 & 1 & 0 & 3 & & & \\
\hline 504 & 1 & $20-30$ & 1 & 2 & 1 & 0 & 3 & & & \\
\hline 504 & 1 & $20-30$ & 1 & 2 & 1 & 12 & 1 & & & \\
\hline 504 & 1 & $20-30$ & 1 & 2 & 1 & 0 & 1 & & & \\
\hline 504 & 1 & $20-30$ & 2 & 8 & 4 & 25 & 1 & & & 6 Flake scars \\
\hline 504 & 1 & $20-30$ & 2 & 6 & 3 & 0 & 1 & & & 10 Flake scars \\
\hline 504 & 1 & $20-30$ & - & - & - & - & - & Limestone \& Chert & & \\
\hline 504 & 1 & $40-50$ & 1 & 3 & 1 & 0 & 1 & & & \\
\hline 504 & 1 & $50-60$ & 1 & 3 & 1 & 0 & 1 & Chert & & \\
\hline 504 & 3 & $0-10$ & 1 & 4 & 2 & 62 & 1 & & & \\
\hline 504 & 3 & $0-10$ & 1 & 3 & 1 & 12 & 1 & & & \\
\hline 504 & 3 & $0-10$ & 1 & 1 & 1 & 0 & 1 & & & \\
\hline 504 & 3 & $0-10$ & 1 & 2 & 1 & 0 & 1 & & & \\
\hline 504 & 3 & $0-10$ & 1 & 2 & 1 & 0 & 1 & & & \\
\hline 504 & 3 & $10-20$ & 1 & 6 & 3 & 38 & 1 & & & \\
\hline 504 & 3 & $10-20$ & 1 & 1 & 1 & 0 & 1 & & & \\
\hline 504 & 3 & $20-30$ & 1 & 3 & 1 & 12 & 1 & & & \\
\hline 504 & 6 & $0-10$ & 7 & - & - & - & - & & & Whiteware sherd \\
\hline 504 & 7 & $0-10$ & 1 & 1 & 1 & 0 & 1 & & & \\
\hline 504 & 7 & $0-10$ & 1 & 1 & 1 & 12 & 4 & & & \\
\hline 504 & 8 & $0-10$ & 1 & 4 & 2 & 12 & 9 & & & \\
\hline 504 & 8 & $0-10$ & 1 & 3 & 2 & 0 & 1 & & & \\
\hline 504 & 8 & $0-10$ & 1 & 2 & 1 & 0 & 1 & & & \\
\hline 504 & 8 & $0-10$ & 6 & 4 & 1 & 12 & 1 & & & \\
\hline 504 & 8 & $0-10$ & 1 & 2 & 1 & 0 & 1 & & & \\
\hline 504 & 8 & $0-10$ & 1 & 3 & 1 & 12 & 1 & & & \\
\hline 504 & 8 & $0-10$ & 1 & 2 & 1 & 0 & 1 & & & \\
\hline 504 & 8 & $10-20$ & 1 & 2 & 1 & 12 & 1 & & & \\
\hline 504 & 8 & $10-20$ & 1 & 2 & 1 & 0 & 1 & & & \\
\hline 504 & 9 & $0-10$ & 4 & 3 & 2 & 0 & 1 & & & Fragment \\
\hline 504 & 9 & $0-10$ & 7 & - & - & - & - & & & .22 caliber cartridge \\
\hline 504 & 9 & $30-40$ & 1 & 2 & 1 & 0 & 1 & & & \\
\hline
\end{tabular}


Table B-1. continued...

\begin{tabular}{|c|c|c|c|c|c|c|c|c|c|c|}
\hline $\begin{array}{r}\text { Site } \\
\text { 41TG } \\
\end{array}$ & $\begin{array}{r}\text { Shovel } \\
\text { Test \# }\end{array}$ & $\begin{array}{c}\text { Depth } \\
\text { cm }\end{array}$ & $\begin{array}{c}\text { Artifact } \\
\text { Type }\end{array}$ & $\begin{array}{l}\text { Max. } \\
\text { Length }\end{array}$ & $\begin{array}{c}\text { Max. } \\
\text { Thickness } \\
\end{array}$ & $\begin{array}{c}\text { Cortex } \\
\%\end{array}$ & \begin{tabular}{|c|} 
Material \\
Color \\
\end{tabular} & $\begin{array}{c}\text { Fire Cracked } \\
\text { Rock }\end{array}$ & $\begin{array}{c}\text { Mussel } \\
\text { Shell }\end{array}$ & $\begin{array}{c}\text { Other / } \\
\text { Remarks }\end{array}$ \\
\hline 506 & 1 & $0-10$ & 1 & 2 & 1 & 0 & 1 & & & \\
\hline 506 & 1 & $10-20$ & - & - & - & - & - & & & 3 Deer bone fragments \\
\hline 506 & 1 & $10-20$ & 1 & 3 & 1 & 0 & 1 & & & \\
\hline 506 & 1 & $10-20$ & 1 & 2 & 1 & 12 & 1 & & & \\
\hline 506 & 1 & $10-20$ & 1 & 2 & 1 & 0 & 1 & & & \\
\hline 506 & 1 & $10-20$ & 1 & 2 & 1 & 0 & 1 & & & \\
\hline 506 & 1 & $10-20$ & 1 & 2 & 1 & 62 & 1 & & & \\
\hline 506 & 1 & $10-20$ & 1 & 1 & 1 & 0 & 1 & & & \\
\hline 506 & 1 & $20-30$ & 1 & 2 & 1 & 12 & 3 & Limestone \& Chert & & \\
\hline 506 & 1 & $20-30$ & 1 & 2 & 1 & 0 & 1 & & & \\
\hline 506 & 1 & $20-30$ & 1 & 2 & 1 & 0 & 1 & & & \\
\hline 506 & 1 & $20-30$ & - & - & - & - & - & & & Bone fragment \\
\hline 506 & 1 & $30-40$ & 1 & 2 & 1 & 0 & 1 & & & Blade \\
\hline 506 & 1 & $30-40$ & 1 & 2 & 1 & 0 & 1 & Chert & & \\
\hline 506 & 1 & $30-40$ & 1 & 2 & 1 & 0 & 1 & & & \\
\hline 506 & 1 & $30-40$ & 1 & 2 & 1 & 0 & 1 & & & \\
\hline 506 & 1 & $50-60$ & 1 & 3 & 1 & 38 & 1 & & & \\
\hline 506 & 1 & $50-60$ & 1 & 2 & 1 & 38 & 1 & & & \\
\hline 506 & 2 & $10-20$ & - & - & - & - & - & Limestone & & \\
\hline 506 & 2 & $20-30$ & 6 & 3 & 1 & 0 & 1 & Limestone & & \\
\hline 506 & 2 & $30-40$ & 1 & 3 & 1 & 0 & 1 & Limestone & & \\
\hline 506 & 6 & $50-60$ & 3 & 8 & 4 & 75 & 1 & & & \\
\hline 506 & 8 & $0-10$ & 1 & 4 & 3 & 12 & 1 & & & \\
\hline 506 & 8 & $0-10$ & 1 & 5 & 1 & 12 & 1 & & & \\
\hline 506 & 8 & $10-20$ & 4 & 5 & 1 & 0 & 4 & & & \\
\hline 506 & 8 & $10-20$ & 1 & 5 & 1 & 38 & 1 & & & \\
\hline 506 & 8 & $20-30$ & 1 & 2 & 1 & 0 & 1 & & & \\
\hline 506 & 8 & $30-40$ & 1 & 4 & 1 & 0 & 1 & & & \\
\hline 506 & 8 & $30-40$ & 1 & 3 & 1 & 0 & 1 & & & \\
\hline 506 & 8 & $30-40$ & 4 & 2 & 1 & 0 & 1 & & & \\
\hline 506 & 8 & $30-40$ & 1 & 3 & 1 & 12 & 3 & & & \\
\hline 506 & 8 & $30-40$ & 1 & 2 & 1 & 100 & 3 & & & \\
\hline 506 & 8 & $30-40$ & 1 & 2 & 1 & 0 & 3 & & & \\
\hline 506 & 8 & $30-40$ & 1 & 2 & 1 & 0 & 1 & & & \\
\hline 506 & 8 & $30-40$ & 1 & 2 & 1 & 12 & 1 & & & \\
\hline 506 & 8 & $30-40$ & 1 & 2 & 1 & 12 & 1 & & & \\
\hline 506 & 8 & $30-40$ & 1 & 2 & 1 & 12 & 3 & & & \\
\hline 506 & 8 & $30-40$ & 1 & 1 & 1 & 100 & 3 & & & \\
\hline 506 & 8 & $30-40$ & - & - & - & - & - & & $x$ & \\
\hline 506 & 8 & $40-50$ & 1 & 4 & 1 & 12 & 4 & & & \\
\hline 506 & 8 & $40-50$ & 1 & 4 & 1 & 12 & 4 & & & \\
\hline 506 & 8 & $40-50$ & 1 & 2 & 1 & 0 & 1 & & & \\
\hline 506 & 8 & $40-50$ & 1 & 2 & 1 & 12 & 1 & & & \\
\hline 506 & 8 & $40-50$ & 1 & 3 & 1 & 88 & 1 & & & \\
\hline 506 & 8 & $40-50$ & 1 & 3 & 1 & 0 & 1 & & & \\
\hline 506 & 8 & $40-50$ & 1 & 2 & 1 & 0 & 3 & & & \\
\hline 506 & 8 & $40-50$ & 1 & 2 & 1 & 0 & 1 & & & \\
\hline
\end{tabular}


Table B-1. continued...

\begin{tabular}{|c|c|c|c|c|c|c|c|c|c|c|}
\hline $\begin{array}{r}\text { Site } \\
\text { 41TG } \\
\end{array}$ & $\begin{array}{l}\text { Shovel } \\
\text { Test \# }\end{array}$ & $\begin{array}{c}\text { Depth } \\
\text { cm }\end{array}$ & $\begin{array}{c}\text { Artifact } \\
\text { Type }\end{array}$ & $\begin{array}{c}\text { Max. } \\
\text { Length }\end{array}$ & $\begin{array}{c}\text { Max. } \\
\text { Thickness }\end{array}$ & $\begin{array}{c}\text { Cortex } \\
\% \\
\end{array}$ & \begin{tabular}{|c|}
$\begin{array}{c}\text { Material } \\
\text { Color }\end{array}$ \\
\end{tabular} & $\begin{array}{c}\begin{array}{c}\text { Fire Cracked } \\
\text { Rock }\end{array} \\
\end{array}$ & $\begin{array}{c}\text { Mussel } \\
\text { Shell } \\
\end{array}$ & $\begin{array}{c}\text { Other / } \\
\text { Remarks }\end{array}$ \\
\hline 506 & 8 & $40-50$ & 1 & 2 & 1 & 0 & 1 & & & \\
\hline 506 & 8 & $40-50$ & 1 & 2 & 1 & 88 & 2 & & & \\
\hline 506 & 8 & $40-50$ & 1 & 2 & 1 & 0 & 4 & & & \\
\hline 506 & 8 & $40-50$ & 1 & 2 & 1 & 0 & 1 & & & \\
\hline 506 & 8 & $40-50$ & 1 & 2 & 1 & 0 & 3 & & & \\
\hline 506 & 8 & $40-50$ & 1 & 2 & 1 & 0 & 1 & & & \\
\hline 506 & 8 & $40-50$ & 1 & 1 & 1 & 0 & 2 & & & \\
\hline 506 & 8 & $40-50$ & 1 & 1 & 1 & 0 & 3 & & & \\
\hline 506 & 8 & $40-50$ & - & - & - & - & - & Limestone & & \\
\hline 506 & 8 & $40-50$ & _. & - & - & - & - & & $\mathrm{x}$ & \\
\hline 506 & 8 & $50-60$ & 2 & 11 & 3 & 12 & 1 & & & \\
\hline 506 & 8 & $50-60$ & 1 & 4 & 1 & 0 & 1 & & & \\
\hline 506 & 8 & $50-60$ & 1 & 3 & 1 & 0 & 1 & & & \\
\hline 506 & 8 & $50-60$ & 6 & 2 & 1 & 0 & 1 & & & Spokeshave \\
\hline 506 & 8 & $50-60$ & 1 & 2 & 1 & 0 & 1 & & & \\
\hline 506 & 8 & $50-60$ & 1 & 2 & 1 & 0 & 1 & & & \\
\hline 506 & 8 & $50-60$ & _. & - & - & - & - & & $\mathrm{x}$ & \\
\hline 506 & 8 & $60-70$ & 1 & 4 & 1 & 0 & 1 & & & \\
\hline 506 & 8 & $60-70$ & 1 & 2 & 1 & 12 & 1 & & & \\
\hline 506 & 8 & $60-70$ & 1 & 3 & 1 & 12 & 1 & & & \\
\hline 506 & 8 & $60-70$ & 6 & 3 & 1 & 12 & 1 & & & \\
\hline 506 & 8 & $60-70$ & 1 & 2 & 1 & 12 & 1 & & & \\
\hline 506 & 8 & $60-70$ & 1 & 2 & 1 & 0 & 1 & & & \\
\hline 506 & 8 & $60-70$ & 1 & 2 & 1 & 0 & 1 & & & \\
\hline 506 & 8 & $60-70$ & - & - & - & - & - & Chert & & \\
\hline 506 & 9 & $10-20$ & 1 & 2 & 1 & 0 & 1 & & & \\
\hline 506 & 9 & $30-40$ & 4 & 8 & 2 & 25 & 1 & & & Crudely worked \\
\hline 506 & 9 & $30-40$ & 1 & 3 & 1 & 0 & 1 & & & \\
\hline 506 & 9 & $30-40$ & 1 & 3 & 1 & 0 & 1 & & & \\
\hline 506 & 9 & $30-40$ & 1 & 2 & 1 & 62 & 1 & & & \\
\hline 506 & 10 & $20-30$ & 1 & 2 & 1 & 0 & 1 & & $\mathrm{x}$ & \\
\hline 506 & 10 & $20-30$ & 1 & 2 & 1 & 0 & 1 & & & \\
\hline 506 & 10 & $20-30$ & 1 & 2 & 1 & 0 & 1 & & & \\
\hline 506 & 10 & $30-40$ & 1 & 2 & 1 & 0 & 1 & & $\mathrm{x}$ & \\
\hline 506 & 10 & $30-40$ & 1 & 2 & 1 & 0 & 1 & & & \\
\hline 506 & 11 & $10-20$ & 1 & 2 & 1 & 0 & 1 & & & \\
\hline 506 & 12 & $20-30$ & 1 & 2 & 1 & 0 & 1 & & & \\
\hline 506 & 12 & $20-30$ & 1 & 2 & 1 & 0 & 1 & & & \\
\hline 506 & 12 & $20-30$ & 1 & 2 & 1 & 12 & 1 & & & \\
\hline 506 & 13 & $0-10$ & 1 & 4 & 1 & 0 & 1 & Limestone & & \\
\hline 506 & 13 & $0-10$ & 1 & 2 & 1 & 0 & 3 & & & \\
\hline 506 & 13 & $10-20$ & 1 & 3 & 1 & 88 & 1 & Chert \& Limestone & & \\
\hline 506 & 13 & $10-20$ & 1 & 2 & 1 & 0 & 1 & & & \\
\hline 506 & 13 & $10-20$ & 1 & 4 & 2 & 12 & 3 & & & \\
\hline 506 & 13 & $20-30$ & 1 & 6 & 1 & 12 & 1 & Chert \& Limestone & & \\
\hline 506 & 13 & $20-30$ & 1 & 3 & 1 & 0 & 1 & & & \\
\hline 506 & 13 & $20-30$ & 1 & 4 & 1 & 0 & 3 & & & \\
\hline
\end{tabular}


Table B-1. continued...

\begin{tabular}{|c|c|c|c|c|c|c|c|c|c|c|}
\hline $\begin{array}{r}\text { Site } \\
41 \mathrm{TG} \\
\end{array}$ & $\begin{array}{l}\text { Shovel } \\
\text { Test \# }\end{array}$ & $\begin{array}{c}\text { Depth } \\
\text { cm }\end{array}$ & $\begin{array}{c}\text { Artifact } \\
\text { Type }\end{array}$ & $\begin{array}{l}\text { Max. } \\
\text { Length }\end{array}$ & $\begin{array}{l}\text { Max. } \\
\text { Thickness }\end{array}$ & $\begin{array}{c}\text { Cortex } \\
\% \\
\end{array}$ & $\begin{array}{c}\text { Material } \\
\text { Color } \\
\end{array}$ & $\begin{array}{c}\text { Fire Cracked } \\
\text { Rock }\end{array}$ & $\begin{array}{c}\text { Mussel } \\
\text { Shell }\end{array}$ & $\begin{array}{c}\text { Other / } \\
\text { Remarks }\end{array}$ \\
\hline 506 & 13 & $20-30$ & 1 & 2 & 1 & 0 & 1 & & & \\
\hline 506 & 13 & $20-30$ & 1 & 2 & 1 & 0 & 1 & & & \\
\hline 506 & 13 & $20-30$ & 1 & 2 & 1 & 12 & 1 & & & \\
\hline 506 & 13 & $30-40$ & 1 & 2 & 1 & 0 & 1 & Chert \& Sandstone & & \\
\hline 506 & 13 & $30-40$ & 1 & 2 & 1 & 0 & 1 & & & \\
\hline 506 & 13 & $30-40$ & 1 & 2 & 1 & 12 & 1 & & & \\
\hline 506 & 13 & $40-50$ & 1 & 2 & 1 & 0 & 1 & Limestone & & \\
\hline 506 & 13 & $50-60$ & 1 & 3 & 1 & 12 & 1 & & & \\
\hline 506 & 15 & $10-20$ & 1 & 4 & 2 & 88 & 1 & & $\mathrm{X}$ & \\
\hline 506 & 15 & $20-30$ & - & - & - & - & - & Chert & $\mathrm{x}$ & \\
\hline 506 & 16 & $20-30$ & 1 & 6 & 1 & 0 & 1 & & & \\
\hline 506 & 16 & $40-50$ & 1 & 3 & 1 & 0 & 1 & & & \\
\hline 506 & 16 & $40-50$ & 1 & 2 & 1 & 0 & 1 & & & \\
\hline 506 & 17 & $0-10$ & 1 & 3 & 1 & 0 & 1 & & & \\
\hline 506 & 17 & $0-10$ & 1 & 2 & 1 & 12 & 2 & & & \\
\hline 506 & 18 & $10-20$ & 1 & 3 & 1 & 0 & 1 & Chert & & \\
\hline 506 & 18 & $10-20$ & 1 & 2 & 1 & 0 & 1 & & & \\
\hline 506 & 18 & $10-20$ & 1 & 2 & 1 & 0 & 1 & & & \\
\hline 506 & 18 & 10-20 & 1 & 2 & 1 & 0 & 1 & & & \\
\hline 506 & 18 & $10-20$ & 1 & 2 & 1 & 12 & 1 & & & \\
\hline 506 & 18 & $20-30$ & 1 & 2 & 1 & 0 & 1 & Limestone & & \\
\hline 506 & 18 & $20-30$ & 1 & 2 & 1 & 0 & 1 & & & \\
\hline 506 & 18 & $20-30$ & 1 & 2 & 1 & 12 & 1 & & & \\
\hline 506 & 18 & $30-40$ & 1 & 2 & 1 & 0 & 1 & Limestone & & 12 Large cobbles \\
\hline 506 & 18 & $30-40$ & 1 & 2 & 1 & 0 & 1 & & & \\
\hline 506 & 18 & $50-60$ & 1 & 2 & 1 & 0 & 1 & & & \\
\hline 506 & Auger & $40-50$ & 1 & 4 & 2 & 0 & 1 & & & \\
\hline 509 & 1 & $0-10$ & 1 & 6 & 1 & 0 & 1 & & & \\
\hline 509 & 1 & $0-10$ & 1 & 4 & 1 & 0 & 1 & & & \\
\hline 509 & 1 & $0-10$ & 1 & 3 & 1 & 0 & 1 & & & \\
\hline 509 & 1 & $0-10$ & 1 & 4 & 1 & 0 & 1 & & & \\
\hline 509 & 1 & $0-10$ & 1 & 3 & 1 & 0 & 1 & & & \\
\hline 509 & 1 & $0-10$ & 1 & 3 & 1 & 62 & 1 & & & \\
\hline 509 & 1 & $0-10$ & 1 & 3 & 1 & 0 & 1 & & & \\
\hline 509 & 1 & $0-10$ & 1 & 2 & 1 & 0 & 1 & & & \\
\hline 509 & 1 & $0-10$ & 1 & 2 & 1 & 0 & 1 & & & \\
\hline 509 & 1 & $0-10$ & 1 & 2 & 1 & 0 & 1 & & & \\
\hline 509 & 1 & $0-10$ & 1 & 2 & 1 & 0 & 1 & & & \\
\hline 509 & 1 & $0-10$ & 1 & 2 & 1 & 0 & 1 & & & \\
\hline 509 & 1 & $0-10$ & 1 & 1 & 1 & 0 & 1 & & & \\
\hline 509 & 1 & $0-10$ & 1 & 2 & 1 & 0 & 1 & & & \\
\hline 509 & 1 & $0-10$ & 1 & 2 & 1 & 0 & 1 & & & \\
\hline 509 & 1 & $0-10$ & 1 & 2 & 1 & 0 & 1 & & & \\
\hline 509 & 1 & $0-10$ & 1 & 2 & 1 & 0 & 1 & & & \\
\hline 509 & 1 & $10-20$ & - & - & - & - & - & Chert \& Limestone & $x$ & Burned Bone \\
\hline 509 & 1 & $10-20$ & 1 & 4 & 1 & 0 & 1 & Chert & & \\
\hline 509 & 1 & $30-40$ & 1 & 4 & 1 & 12 & 1 & & $x$ & \\
\hline
\end{tabular}


Table B-1. continued...

\begin{tabular}{|c|c|c|c|c|c|c|c|c|c|c|}
\hline $\begin{array}{r}\text { Site } \\
\text { 41TG } \\
\end{array}$ & $\begin{array}{l}\text { Shovel } \\
\text { Test \# }\end{array}$ & $\begin{array}{c}\text { Depth } \\
\text { cm }\end{array}$ & \begin{tabular}{|c}
$\begin{array}{c}\text { Artifact } \\
\text { Tvpe }\end{array}$ \\
\end{tabular} & $\begin{array}{c}\text { Max. } \\
\text { Length }\end{array}$ & $\begin{array}{c}\text { Max. } \\
\text { Thickness }\end{array}$ & $\begin{array}{c}\text { Cortex } \\
\% \\
\end{array}$ & \begin{tabular}{|c|} 
Material \\
Color \\
\end{tabular} & $\begin{array}{c}\text { Fire Cracked } \\
\text { Rock }\end{array}$ & $\begin{array}{c}\text { Mussel } \\
\text { Shell }\end{array}$ & $\begin{array}{c}\text { Other / } \\
\text { Remarks }\end{array}$ \\
\hline 509 & 1 & $40-50$ & 1 & 4 & 1 & 0 & 1 & Chert \& Limestone & & \\
\hline 509 & 1 & $50-60$ & - & - & - & - & - & Sandstone & & \\
\hline 509 & 2 & $20-30$ & - & - & - & - & - & Limestone & & Charcoal \\
\hline 509 & 2 & $40-50$ & - & - & - & - & - & Limestone & & \\
\hline 509 & 3 & $0-10$ & 1 & 4 & 1 & 12 & 1 & & & \\
\hline 509 & 3 & $0-10$ & 1 & 2 & 1 & 0 & 1 & & & \\
\hline 509 & 3 & $30-40$ & 1 & 4 & 3 & 62 & 1 & & & \\
\hline 509 & 4 & $0-10$ & 1 & 1 & 2 & 0 & 1 & Limestone & & \\
\hline 509 & 4 & $0-10$ & 6 & 4 & 1 & 12 & 1 & Chert \& Limestone & $\mathrm{X}$ & Charred pecan shell \\
\hline 509 & 4 & $0-10$ & 1 & 3 & 1 & 12 & 1 & & & \\
\hline 509 & 4 & $0-10$ & 1 & 4 & 1 & 12 & 1 & & & \\
\hline 509 & 4 & $10-20$ & 1 & 5 & 1 & 0 & 1 & Limestone & & \\
\hline 509 & 4 & $20-30$ & 1 & 3 & 1 & 0 & 1 & Limestone & & \\
\hline 509 & 4 & $20-30$ & 1 & 6 & 2 & 88 & 1 & & & \\
\hline 509 & 4 & $30-40$ & - & - & - & - & - & Chert \& Limestone & $\mathrm{X}$ & \\
\hline 509 & 4 & $40-50$ & 7 & 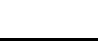 & 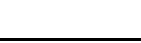 & 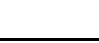 & 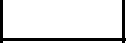 & Limestone & & Not Collected \\
\hline 509 & 5 & $0-10$ & 1 & 5 & 1 & 38 & 1 & & & \\
\hline 509 & 5 & $0-10$ & 1 & 3 & 1 & 0 & 1 & & & \\
\hline 509 & 5 & $0-10$ & 4 & 2 & 1 & 0 & 1 & & & \\
\hline 509 & 5 & $0-10$ & 1 & 2 & 1 & 0 & 3 & & & \\
\hline 509 & 5 & $0-10$ & 1 & 4 & 2 & 12 & 3 & & & \\
\hline 509 & 5 & $0-10$ & 1 & 1 & 1 & 0 & 1 & & & \\
\hline 509 & 5 & $0-10$ & 1 & 2 & 1 & 0 & 1 & & & \\
\hline 509 & 5 & $0-10$ & 1 & 2 & 1 & 0 & 1 & & & \\
\hline 509 & 5 & $0-10$ & - & - & - & - & - & Limestone & & \\
\hline 509 & 5 & $0-10$ & _. & - & _. & - & - & Sandstone & & \\
\hline 509 & 5 & $0-10$ & 7 & - & - & - & - & & $\mathrm{x}$ & \\
\hline 509 & 5 & $0-10$ & 7 & - & - & - & - & & & Bone \\
\hline 509 & 5 & $10-20$ & - & - & - & - & - & Limestone & & \\
\hline 509 & 5 & $10-20$ & 1 & 3 & 1 & 12 & - & & & \\
\hline 509 & 5 & $10-20$ & 1 & 1 & 1 & 0 & 1 & & & \\
\hline 509 & 5 & $10-20$ & 1 & 2 & 1 & 0 & 1 & & & \\
\hline 509 & 5 & $20-30$ & 1 & 2 & 1 & 0 & 1 & & & \\
\hline 509 & 6 & $30-40$ & - & - & - & - & - & Limestone & & \\
\hline 509 & 6 & $30-40$ & 1 & 2 & 1 & 0 & 1 & & & \\
\hline 510 & 1 & $40-50$ & 1 & 3 & 1 & 0 & 1 & & & \\
\hline 510 & 6 & $20-30$ & 1 & 2 & 1 & 0 & 2 & & & \\
\hline 510 & 6 & $50-60$ & 1 & 3 & 1 & 0 & 1 & & & \\
\hline 511 & 2 & $10-20$ & 1 & 2 & 1 & 0 & 1 & & $\mathrm{X}$ & \\
\hline 511 & 7 & $0-10$ & 1 & 2 & 1 & 38 & 1 & & & \\
\hline 512 & 1 & $0-10$ & 1 & 4 & 2 & 0 & 1 & & & \\
\hline 512 & 1 & $0-10$ & 4 & 5 & 1 & 0 & 1 & & & Preform fragment \\
\hline 512 & 1 & $0-10$ & 1 & 2 & 1 & 100 & 3 & & & \\
\hline 512 & 1 & $0-10$ & 1 & 2 & 1 & 0 & 3 & & & \\
\hline 512 & 2 & $0-10$ & 1 & 3 & 1 & 0 & 1 & & & \\
\hline 512 & 2 & $0-10$ & 1 & 3 & 1 & 0 & 1 & & & \\
\hline 512 & 2 & $0-10$ & 1 & 4 & 2 & 12 & 1 & & & \\
\hline
\end{tabular}


Table B-1. continued...

\begin{tabular}{|c|c|c|c|c|c|c|c|c|c|c|}
\hline $\begin{array}{r}\text { Site } \\
41 T G \\
\end{array}$ & $\begin{array}{l}\text { Shovel } \\
\text { Test \# }\end{array}$ & $\begin{array}{c}\text { Depth } \\
\text { cm }\end{array}$ & $\begin{array}{c}\text { Artifact } \\
\text { Type }\end{array}$ & $\begin{array}{l}\text { Max. } \\
\text { Length }\end{array}$ & $\begin{array}{c}\text { Max. } \\
\text { Thickness }\end{array}$ & $\begin{array}{c}\text { Cortex } \\
\%\end{array}$ & \begin{tabular}{|c|} 
Material \\
Color \\
\end{tabular} & $\begin{array}{c}\text { Fire Cracked } \\
\text { Rock }\end{array}$ & $\begin{array}{c}\begin{array}{c}\text { Mussel } \\
\text { Shell }\end{array} \\
\end{array}$ & $\begin{array}{c}\text { Other / } \\
\text { Remarks }\end{array}$ \\
\hline 513 & 1 & $20-30$ & 4 & 4 & 1 & 0 & 2 & Limestone & & \\
\hline 513 & 1 & $30-40$ & 4 & 8 & 2 & 12 & 2 & & & \\
\hline 513 & 2 & $0-10$ & 1 & 4 & 1 & 0 & 1 & & & \\
\hline 513 & 2 & $0-10$ & 1 & 2 & 1 & 0 & 1 & & & \\
\hline 513 & 2 & $0-10$ & 1 & 2 & 1 & 0 & 1 & & & \\
\hline 513 & 2 & $0-10$ & 1 & 2 & 1 & 0 & 1 & & & \\
\hline 513 & 2 & $0-10$ & 1 & 2 & 1 & 0 & 1 & & & \\
\hline 513 & 2 & $0-10$ & 1 & 2 & 1 & 0 & 1 & & & \\
\hline 513 & 2 & $0-10$ & 1 & 2 & 1 & 0 & 1 & & & \\
\hline 513 & 2 & $0-10$ & 1 & 1 & 1 & 0 & 1 & & & \\
\hline 513 & 2 & $0-10$ & 1 & 1 & 1 & 0 & 1 & & & \\
\hline 513 & 2 & $0-10$ & 1 & 1 & 1 & 0 & 1 & & & \\
\hline 513 & 2 & $0-10$ & 1 & 1 & 1 & 0 & 1 & & & \\
\hline 513 & 2 & $0-10$ & 1 & 2 & 1 & 12 & 2 & & & \\
\hline 513 & 2 & $0-10$ & 1 & 2 & 1 & 38 & 1 & & & \\
\hline 513 & 2 & $0-10$ & 1 & 1 & 1 & 0 & 1 & & & \\
\hline 513 & 2 & $10-20$ & 1 & 2 & 1 & 0 & 1 & & & \\
\hline 513 & 2 & $10-20$ & 1 & 2 & 1 & 0 & 1 & & & \\
\hline 513 & 2 & $10-20$ & 1 & 2 & 1 & 0 & 1 & & & \\
\hline 513 & 2 & $10-20$ & 1 & 3 & 1 & 0 & 1 & & & \\
\hline 513 & 3 & $0-10$ & 1 & 2 & 1 & 0 & 1 & & & \\
\hline 513 & 3 & $0-10$ & 1 & 3 & 1 & 12 & 1 & & & \\
\hline 513 & 3 & $10-20$ & 1 & 6 & 2 & 38 & 1 & & & \\
\hline 513 & 4 & $0-10$ & 1 & 4 & 3 & 12 & 1 & & & \\
\hline 513 & 4 & $0-10$ & 1 & 3 & 1 & 0 & 1 & & & \\
\hline 513 & 4 & $0-10$ & 1 & 2 & 1 & 0 & 1 & & & \\
\hline 513 & 5 & $0-10$ & 6 & 3 & 1 & 0 & 1 & & & \\
\hline 516 & 1 & $40-50$ & 4 & 9 & 2 & 25 & 1 & & & Crudely flaked \\
\hline 516 & 1 & $40-50$ & 1 & 4 & 1 & 0 & 1 & & & \\
\hline 516 & 1 & $40-50$ & 1 & 3 & 1 & 38 & 1 & & & \\
\hline 516 & 1 & $40-50$ & 1 & 2 & 1 & 0 & 1 & & & \\
\hline 516 & 1 & $50-60$ & 1 & 2 & 1 & 0 & 1 & & & \\
\hline 516 & 3 & $10-20$ & 1 & 2 & 1 & 0 & 1 & & & \\
\hline 516 & 4 & $20-30$ & 1 & 2 & 1 & 12 & 1 & & & \\
\hline 516 & 4 & $20-30$ & 1 & 2 & 1 & 0 & 1 & & & \\
\hline 516 & 4 & $20-30$ & 1 & 2 & 1 & 0 & 1 & & & \\
\hline 516 & 5 & $10-20$ & 1 & 2 & 1 & 12 & 1 & & & \\
\hline 516 & 5 & $10-20$ & 1 & 2 & 1 & 0 & 1 & & & \\
\hline 516 & 5 & $10-20$ & 1 & 2 & 1 & 0 & 3 & & & \\
\hline 516 & 6 & $20-30$ & 4 & 3 & 1 & 0 & 2 & & & Distal end \\
\hline 516 & 7 & $0-10$ & 1 & 3 & 1 & 0 & 1 & & & Printed whiteware sherd \\
\hline 516 & 7 & $0-10$ & 1 & 2 & 1 & 0 & 1 & Limestone & & \\
\hline 516 & 7 & $0-10$ & 1 & 1 & 1 & 0 & 1 & & & \\
\hline 516 & 7 & $0-10$ & 1 & 2 & 1 & 12 & 1 & & & \\
\hline 516 & 7 & $0-10$ & 1 & 3 & 1 & 12 & 1 & & & \\
\hline 516 & 7 & $10-20$ & 1 & 2 & 1 & 0 & 1 & & $x$ & Rusted metal fragment \\
\hline 516 & 7 & $40-50$ & 1 & 3 & 1 & 0 & 3 & & & \\
\hline
\end{tabular}


Table B-1. continued...

\begin{tabular}{|c|c|c|c|c|c|c|c|c|c|c|}
\hline $\begin{array}{r}\text { Site } \\
\text { 41TG } \\
\end{array}$ & $\begin{array}{l}\text { Shovel } \\
\text { Test \# }\end{array}$ & $\begin{array}{c}\text { Depth } \\
\text { cm }\end{array}$ & \begin{tabular}{|c}
$\begin{array}{c}\text { Artifact } \\
\text { Tvpe }\end{array}$ \\
\end{tabular} & $\begin{array}{c}\text { Max. } \\
\text { Length }\end{array}$ & $\begin{array}{c}\text { Max. } \\
\text { Thickness }\end{array}$ & $\begin{array}{c}\text { Cortex } \\
\% \\
\end{array}$ & \begin{tabular}{|c|} 
Material \\
Color \\
\end{tabular} & $\begin{array}{c}\begin{array}{c}\text { Fire Cracked } \\
\text { Rock }\end{array} \\
\end{array}$ & $\begin{array}{c}\text { Mussel } \\
\text { Shell } \\
\end{array}$ & $\begin{array}{c}\text { Other / } \\
\text { Remarks }\end{array}$ \\
\hline 516 & 7 & $40-50$ & 1 & 2 & 1 & 0 & 1 & & & \\
\hline 516 & 7 & $40-50$ & 1 & 2 & 1 & 88 & 1 & & & \\
\hline 516 & 8 & $0-10$ & - & - & - & - & - & & & Purple \& clear glass \\
\hline 516 & 9 & $10-20$ & 1 & 5 & 2 & 88 & 1 & & & \\
\hline 517 & 2 & $0-10$ & 1 & 2 & 1 & 0 & 1 & & & \\
\hline 517 & 3 & $0-10$ & 1 & 2 & 1 & 12 & 1 & & & \\
\hline 518 & 1 & $20-30$ & 1 & 2 & 1 & 12 & 1 & & & \\
\hline 524 & 1 & $0-10$ & 1 & 2 & 1 & 12 & 1 & & & \\
\hline 524 & 1 & $0-10$ & 1 & 2 & 1 & 100 & 1 & & & \\
\hline 524 & 3 & $10-20$ & 1 & 3 & 1 & 100 & 1 & & & \\
\hline 524 & 7 & $0-10$ & 1 & 3 & 1 & 100 & 1 & & & \\
\hline 524 & 7 & $0-10$ & 1 & 2 & 1 & 88 & 1 & & & \\
\hline 524 & 7 & $0-10$ & 1 & 2 & 1 & 0 & 1 & & & \\
\hline 524 & 8 & $0-10$ & 6 & 5 & 1 & 12 & 1 & & & \\
\hline 524 & 19 & $20-30$ & 1 & 4 & 1 & 38 & 1 & & & \\
\hline 524 & 19 & $20-30$ & 1 & 3 & 1 & 12 & 1 & & & \\
\hline 524 & 19 & $20-30$ & 1 & 3 & 1 & 12 & 1 & & & \\
\hline 524 & 22 & $0-10$ & 3 & 6 & 2 & 25 & 1 & & & \\
\hline 524 & 22 & $0-10$ & 1 & 1 & 1 & 0 & 1 & & & \\
\hline 524 & 22 & $0-10$ & 1 & 2 & 1 & 100 & 1 & & & \\
\hline 524 & 22 & $0-10$ & 1 & 2 & 1 & 88 & 1 & & & \\
\hline 524 & 22 & $0-10$ & 1 & 3 & 1 & 12 & 1 & & & \\
\hline 525 & 2 & $0-10$ & 1 & 2 & 1 & 0 & 1 & & & Brown beer bottle glass \\
\hline 525 & 2 & $20-30$ & 1 & 2 & 1 & 0 & 1 & & $\mathrm{X}$ & \\
\hline 525 & 2 & $20-30$ & 1 & 3 & 1 & 0 & 1 & & & \\
\hline 525 & 2 & $30-40$ & 1 & 3 & 1 & 0 & 1 & & & \\
\hline 525 & 2 & $30-40$ & 1 & 3 & 1 & 12 & 1 & & & \\
\hline 525 & 3 & $40-50$ & 1 & 2 & 1 & 0 & 1 & & & \\
\hline 525 & 5 & $30-40$ & 4 & 6 & 2 & 0 & 1 & & & Crudely worked \\
\hline 525 & 5 & $30-40$ & 1 & 3 & 1 & 88 & 1 & & & \\
\hline 525 & 5 & $30-40$ & 1 & 3 & 1 & 62 & 1 & & & \\
\hline 525 & 5 & $30-40$ & 1 & 2 & 1 & 88 & 1 & & & \\
\hline 525 & 5 & $30-40$ & 1 & 3 & 1 & 88 & 1 & & & \\
\hline 525 & 5 & $30-40$ & 1 & 2 & 1 & 62 & 1 & & & \\
\hline 525 & 5 & $30-40$ & 1 & 2 & 1 & 0 & 1 & & & \\
\hline 526 & 2 & $0-10$ & 1 & 1 & 1 & 12 & 1 & & & \\
\hline 526 & 2 & $30-40$ & 1 & 3 & 1 & 62 & 1 & & & \\
\hline 526 & 2 & $40-50$ & 1 & 3 & 1 & 12 & 1 & & & \\
\hline 526 & 5 & $50-60$ & 1 & 3 & 1 & 0 & 1 & & & \\
\hline 526 & 7 & $10-20$ & 1 & 6 & 2 & 38 & 1 & & & \\
\hline 526 & 7 & $10-20$ & 1 & 2 & 1 & 100 & 4 & & & \\
\hline 526 & 7 & $10-20$ & 1 & 2 & 1 & 0 & 1 & & & \\
\hline 526 & 14 & $10-20$ & 1 & 3 & 1 & 100 & 1 & & & \\
\hline 526 & 14 & $10-20$ & 1 & 2 & 1 & 0 & - & & & \\
\hline 526 & 14 & $20-30$ & 1 & 2 & 1 & 12 & 1 & & & \\
\hline 526 & 16 & $20-30$ & 1 & 2 & 1 & 100 & 1 & & & \\
\hline 526 & 16 & $20-30$ & 1 & 4 & 1 & 12 & 1 & & & \\
\hline
\end{tabular}


Table B-1. continued...

\begin{tabular}{|c|c|c|c|c|c|c|c|c|c|c|}
\hline $\begin{array}{r}\text { Site } \\
41 \mathrm{TG} \\
\end{array}$ & $\begin{array}{l}\text { Shovel } \\
\text { Test \# }\end{array}$ & $\begin{array}{c}\text { Depth } \\
\text { cm }\end{array}$ & $\begin{array}{c}\text { Artifact } \\
\text { Tvpe }\end{array}$ & $\begin{array}{c}\text { Max. } \\
\text { Length }\end{array}$ & $\begin{array}{c}\text { Max. } \\
\text { Thickness } \\
\end{array}$ & $\begin{array}{c}\text { Cortex } \\
\% \\
\end{array}$ & $\begin{array}{c}\text { Material } \\
\text { Color }\end{array}$ & $\begin{array}{c}\begin{array}{c}\text { Fire Cracked } \\
\text { Rock }\end{array} \\
\end{array}$ & $\begin{array}{c}\text { Mussel } \\
\text { Shell } \\
\end{array}$ & $\begin{array}{c}\text { Other / } \\
\text { Remarks }\end{array}$ \\
\hline 526 & 16 & $20-30$ & 1 & 1 & 1 & 12 & 1 & & & \\
\hline 526 & 17 & $40-50$ & 1 & 2 & 1 & 0 & 1 & & & \\
\hline 531 & 1 & $0-10$ & 1 & 2 & 1 & 88 & 1 & & & \\
\hline 531 & 1 & $0-10$ & 1 & 2 & 1 & 88 & 1 & & & \\
\hline 531 & 1 & $0-10$ & 1 & 2 & 1 & 0 & 1 & & & \\
\hline 531 & 1 & $20-30$ & 1 & 3 & 1 & 0 & 1 & & & \\
\hline 531 & 1 & $20-30$ & 1 & 2 & 1 & 12 & 1 & & & \\
\hline 531 & 1 & $20-30$ & 1 & 2 & 1 & 12 & 1 & & & \\
\hline 531 & 1 & $20-30$ & 1 & 4 & 1 & 12 & 1 & & & \\
\hline 531 & 1 & $40-50$ & 6 & 5 & 1 & 0 & 2 & & & \\
\hline 531 & 2 & $0-10$ & 1 & 2 & 1 & 0 & 1 & Chert & & \\
\hline 531 & 2 & $0-10$ & 1 & 2 & 1 & 0 & 1 & & & \\
\hline 531 & 2 & $0-10$ & 1 & 2 & 1 & 0 & 2 & & & \\
\hline 531 & 2 & $0-10$ & 1 & 1 & 1 & 0 & 1 & & & \\
\hline 531 & 4 & $10-20$ & 6 & 3 & 1 & 0 & 1 & & & \\
\hline 532 & 1 & $0-10$ & 1 & 2 & 1 & 0 & 1 & & & \\
\hline 532 & 1 & $0-10$ & 1 & 2 & 1 & 12 & 1 & & & \\
\hline 532 & 1 & $0-10$ & 1 & 2 & 1 & 0 & 1 & & & \\
\hline 532 & 2 & $0-10$ & 1 & 2 & 1 & 0 & 4 & & & \\
\hline 532 & 2 & $0-10$ & 1 & 3 & 1 & 38 & 3 & & & \\
\hline 532 & 2 & $0-10$ & 1 & 2 & 1 & 0 & 3 & & & \\
\hline 533 & 3 & $10-20$ & 1 & 2 & 1 & 12 & 1 & & & \\
\hline 533 & 3 & $10-20$ & 1 & 3 & 1 & 38 & 1 & & & \\
\hline 534 & 3 & $0-10$ & 1 & 3 & 1 & 0 & 1 & & & \\
\hline 534 & 3 & $20-30$ & 1 & 2 & 1 & 0 & 1 & & & \\
\hline 534 & 6 & $10-20$ & 1 & 2 & 1 & 0 & 1 & Limestone & & \\
\hline 534 & 6 & $30-40$ & - & - & - & - & - & Limestone & & \\
\hline 535 & 2 & $0-10$ & 1 & 3 & 1 & 0 & 1 & & & \\
\hline 540 & 3 & $0-10$ & 1 & 4 & 1 & 12 & 1 & & & \\
\hline
\end{tabular}




\title{
Appendix C: Twin Buttes Archaeological Project
}

\author{
Excavation Summary
}

\section{Introduction}

In addition to shovel testing, four previously unrecorded sites were selected for further testing. Two $1-\mathrm{m}^{2}$ test units were excavated at 41TG378 to evaluate the integrity of an early Paleoindian component; one unit each at 41TG389 and 41TG410 to evaluate the integrity of possible Late Archaic components; and one unit at 41TG443 to investigate the remains of a probable historic dugout. This appendix provides the results of those investigations. For a detailed description of each site and the results of preliminary shovel testing and surface observations, the reader is referred to Appendices A, B, and D for more information about the three prehistoric sites, and to Chapter 11 for more information about $41 \mathrm{TG} 443$.

\section{Methods}

All test excavation units were $1 \mathrm{~m}^{2}$ except for the single unit at 41TG443 (Historic component) which was excavated in feet and inches. Excavation was accomplished with trowels, picks and brushes in arbitrary $10 \mathrm{~cm}$ intervals in the prehistoric components, but by stratigraphic levels in the historic component. All sediments were screened through $1 / 4$-inch wire mesh. Cultural material other than special samples were placed in paper bags labeled with the site number, provenience, date, and name of excavator(s). Charcoal samples were placed in aluminum foil, and flotation samples were placed in plastic bags. Photographs using color print and slide film were taken. Photographs were recorded on standard photo logs in the field. In addition to photographing general excavation activities, particular attention was given to features, profiles, and plan views.

Artifacts collected in the field were brought to the Center for Archaeological Research (CAR) laboratory on the UTSA campus. Processing of cultural material recovered began with washing and sorting into categories. Individual lithics were sorted by a size chart and counted. The data were entered on a spreadsheet to facilitate distribution analysis. Spreadsheets were designed to sum the various types of artifacts and to calculate relative percentages.

Acid-free labels were placed in all artifact archival-quality bags for curation. Each bag was labeled with a provenience. Stone tools were labeled with permanent ink and covered by a clear coat of acrylic. Artifact bags were separated by class and stored in acid-free boxes. Boxes were labeled with standard labels.

A single flotation sample of 15 liters of sediments was collected from Feature 1, 41TG389 and transported back to San Antonio for processing at the CAR lab. The sediments were poured into plastic buckets, clean water added, and the mixture gently stirred by hand to bring the light fraction to the water's surface. The floated material was then gently skimmed off the surface or poured through a tightly woven chiffon cloth fitted into a fine wire mesh kitchen 
colander. The cloth with the light fraction on it was then removed and allowed to dry indoors. After drying, the light fraction was poured from the chiffon cloth through graduated nesting screen sizes of $2 \mathrm{~mm}, 1 \mathrm{~mm}$, and $0.5 \mathrm{~mm}$ respectively. A catchment pan was placed on the very bottom to catch any remains finer than $0.5 \mathrm{~mm}$. Any examination of the processed light fractions was done under sterile conditions. The light fraction was then placed in paper letter envelopes and sent to Dr. Philip Dering of Texas A\&M University.

All fire-cracked rock from Feature 1, 41TG389 was collected, brought back to the lab, and was discarded after analysis. No wood charcoal samples were recovered from any of the unit excavations.

Field notes, forms, photographs, and drawings were placed in labeled notebooks. Photographs, slides, and negatives were placed in archival-quality sleeves. All notebooks were stored in acid-free boxes. A copy of the site report and all computer disks pertaining to the investigation of the excavations are stored in an archival box and curated with the field notes and documents. Artifacts, notes, documents, and photographs are permanently housed at the Center for Archaeological Research on The University of Texas at San Antonio campus.

CAR collected 11 core samples from fire-cracked sandstone cobbles in a single feature using a portable rock drill. The cores were oriented according to established paleomagnetic techniques by means of a compass mounted on a goniometer. The elevation of each sample relative to the unit datum was recorded and all samples were identified on a planview map. All samples were analyzed in the Paleomagnetic Laboratory, Department of Geological Sciences, The University of Texas at Austin. All samples were subjected to progressive thermal demagnetization in $50^{\circ} \mathrm{C}$ steps from $150^{\circ} \mathrm{C}$ to $500^{\circ} \mathrm{C}$. The characteristic directions of magnetization were obtained after inspection of the data in orthogonal vector projections and principal component analysis (e.g., Butler 1992).

Samples for magnetic susceptibility studies were collected in four vertical profiles from three excavation units. For each sample column, a $10-\mathrm{cm}$ vertical face of the unit wall was scraped horizontally with a clean trowel to provide a fresh exposure and avoid possible contamination in the open unit. A tape measure was placed vertically on the trench wall and a $>2 \mathrm{~cm}^{3}$ sample of sediment was taken with a trowel every $3 \mathrm{~cm}$ down the face of the unit. The trowel was wiped clean after each sample was taken in order to obtain uncontaminated samples. Samples were collected in plastic bags which were labeled with provenience information. In the laboratory, the samples were placed in 2- $\mathrm{cm}^{3}$ plastic cubes for measurement in a Bartington (MS2) Magnetic Susceptibility Meter.

Sites Excavated

\section{$41 \mathrm{TG} 378$}

\section{Discussion}

A Clovis point base with probable associated debitage, and possibly associated hearth features were found on the surface during the initial survey. Limited shovel testing indicated the potential for buried, intact deposits and thus two $1-\mathrm{m}^{2}$ units were excavated at the site. In addition to the two test units, soil susceptibility samples were collected and processed at the Paleomagnetism Laboratory, The University of Texas at Austin (see Appendix J).

\section{Results and Analysis}

Test Unit 1 was placed at N992 E991, near the discovery of a Clovis point base on the surface (Figure A-20), and was excavated to a depth of $1.1 \mathrm{~m}$. No prehistoric artifacts were recovered. The recovery of modern glass and a 
fishing weight in the upper $20 \mathrm{~cm}$ provided evidence of recent disturbance. The results of a vertical soil susceptibility column analysis taken from the north wall provided no indication of a buried occupation surface.

Test Unit 2 was placed upslope from Test Unit 1, at N1028 E1012. One incomplete flake, one utilized flake, and an indeterminate piece of chert debitage were found in the upper $10 \mathrm{~cm}$. However, modern glass found in the same level provided evidence of recent disturbance. Although two additional incomplete flakes and an indeterminate piece of chert were found between $10-20 \mathrm{~cm}$ below the surface, they were lying just above sloping bedrock. Excavation was terminated $15-40 \mathrm{~cm}$ below the surface on the sloping sandstone bedrock.

Because of the apparent disturbed nature of the flakes found in Unit 2, the absence of cultural material in Unit 1, the lack of evidence for a buried soil in the magnetic susceptibility results (Appendix J), and the geomorphological investigations which indicate that the site is located on a deflated hill slope (see Appendix I), no further work is recommended.

\section{TG389}

\section{Discussion}

During the shovel testing phase a Late Archaic-like dart point was recovered from Shovel Test 1 between 0-10 $\mathrm{cm}$ below the surface, and all six shovel tests contained artifacts between $0-30 \mathrm{~cm}$. Fire-cracked rock was found in Shovel Test 3 and it also yielded a higher density of flakes relative to the other shovel tests (see Appendix B), thus a single $1-\mathrm{m}^{2}$ test unit (N988 E985) was placed nearby (Figure A-31). Archaeomagnetic samples were collected from a fire-cracked rock feature, and soil susceptibility column samples were collected from the east and west walls of the unit.

Results and Analysis

Vertical Distribution of Cultural Remains

A single, complete, ovate-shaped biface measuring $52.61 \mathrm{~mm}$ long, $30.50 \mathrm{~mm}$ wide, and $12.43 \mathrm{~mm}$ thick was found between $30-40 \mathrm{~cm}$ below the surface, just above sloping bedrock. It was made from a cobble of finegrained chert with a few inclusions, and had been heat-treated. It showed evidence of having been reduced to a middle stage of reduction. In addition to the biface, a total of 684 flakes, and two potlids, were also recovered from the single excavation unit. The raw data from the analysis of flakes can be found in Table C-1. Table C-1 provides a summary of mean flake length by depth compared with the Holocene eolian undifferentiated soils recorded by Nordt (see Appendix I).

Table C-1. Vertical distribution of flakes and soils from 41TG389

\begin{tabular}{|c|c|l|}
\hline Depth (cm bs) & $\infty$ Flake Length (mm) & Soil Description \\
\hline $0-6$ & $\mathbf{1 9 . 4 5}(\mathrm{n}=101)$ & $\begin{array}{l}\text { Fine sandy loam, friable, moderate, medium subangular } \\
\text { blocky }\end{array}$ \\
\hline $6-10$ & $18.41(\mathrm{n}=238)$ & Same \\
\hline $10-20$ & $17.24(\mathrm{n}=196)$ & Same, with carbonate clasts \\
\hline $20-30$ & $14.63(\mathrm{n}=98)$ & Same, with carbonate clasts \\
\hline $30-40$ & $\mathbf{1 6 . 2 0}(\mathrm{n}=20)$ & $\begin{array}{l}\text { Sandy clay loam, firm, moderate, medium, coarse } \\
\text { subangular blocky }\end{array}$ \\
\hline $40-50$ & $15.02(\mathrm{n}=24)$ & Same \\
\hline $50-60$ & $\mathbf{1 8 . 0 9}(\mathrm{n}=7)$ & Carbonate cemented channel gravels at $59 \mathrm{~cm}$ \\
\hline
\end{tabular}


Vertical movement of artifacts through layered sediment and soil packages due to size sorting can be problematic in sandy soils (e.g., Stevenson 1991; Vierra 1998). Basically, larger artifacts tend to remain relatively in place, while smaller artifacts tend to move downward commensurate with their decreasing size. Thus, when examined vertically we would expect that larger artifacts represent an occupation zone, with the increasingly smaller artifacts working their way downward below the occupation zone. At other sites in south and central Texas, Vierra (1998), Nickels et al. (1998:91-92), and Nickels (2000) have examined the vertical movement of artifacts through soil horizons, and they have successfully correlated the data with occupation levels.

Table C-1 suggests the presence of human occupation between $0-6 \mathrm{~cm}$ below the surface, within the friable sandy loam. The decreasing mean flake length to $30 \mathrm{~cm}$ below the surface implies size sorting. Nordt (Appendix I) has identified a clear, smooth lower boundary to the friable sandy loam about $29 \mathrm{~cm}$ below the surface, with a transition to a firm, sandy clay loam, and the increase in mean flake length to $16.2 \mathrm{~mm}$ between $30-40 \mathrm{~cm}$ corroborates the implication of a possible occupation surface. Finally, although the numbers are much smaller, the increased mean length in the table also suggest a possible occupation zone between $50-60 \mathrm{~cm}$.

\section{Feature 1}

Feature 1 was a cluster of fire-cracked sandstone cobbles found between $6-17 \mathrm{~cm}$ below the surface, concentrated in the southeastern portion of the unit. Chert debitage and a few mussel shell fragments were found in association, but no charcoal. Eleven core samples drilled and analyzed indicated that the feature was not in situ; presumably the rocks represent discard from a perhaps larger feature to the southeast.

The limited data available from both the shovel tests and single excavation unit indicate that the site still maintains a high degree of integrity and with additional investigations there is a high probability of finding buried, intact cultural features and discrete zones which have the potential to provide valuable information on site formation processes, subsistence, and settlement patterns.

\section{$41 \mathrm{TG} 410$}

\section{Discussion}

During initial testing, a Frio (Late Archaic) dart point was found in Shovel Test 3 between $40-50 \mathrm{~cm}$ below the surface (see Appendix F), and a single radiocarbon assay obtained from charcoal found between 30-40 cm

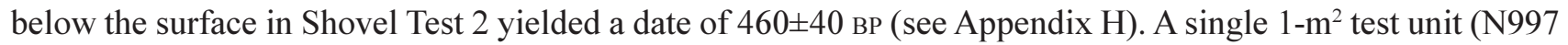
E992) was placed adjacent to the shovel test (Figure A-53) to investigate the possibility of intact features and other cultural material in association with the point. The $1-\mathrm{m}^{2}$ test unit was excavated to $1 \mathrm{~m}$ below the surface.

\section{Results and Analysis}

A total of ten flakes were recovered from the unit, but no fire-cracked rock or other cultural material. All of the flakes were made from chert and none exhibited evidence of heat treating; their attributes are shown in Table C2. The sample size is extremely small and thus any attempted analysis is problematic. However, the number of flakes with multi-faceted platforms, no cortex, and multiple dorsal scars suggests that middle to late stage bifacial reduction activities were occurring at the site. The presence of a rejuvenation flake and an expedient, utilized flake suggest at least a short-term domestic occupation during which some food processing may have occurred. The continuum of recovered flakes between $30-90 \mathrm{~cm}$ did not provide a firm indication of distinct, stratified occupation levels, but does suggest that the site may have been occupied either continuously or intermittently for an extended period of time. 
Table C-2. Attributes of flakes recovered from 41TG410

\begin{tabular}{|c|c|c|c|c|c|c|l|}
\hline $\begin{array}{c}\text { Depth } \\
(\mathbf{c m} \text { bs) }\end{array}$ & Complete? & $\begin{array}{c}\text { Length } \\
(\mathbf{m m})\end{array}$ & $\begin{array}{c}\text { Thickness } \\
(\mathbf{m m})\end{array}$ & $\begin{array}{c}\text { \# Platform } \\
\text { Facets }\end{array}$ & $\begin{array}{c}\text { Cortex } \\
\mathbf{\%}\end{array}$ & $\begin{array}{c}\text { \# Dorsal } \\
\text { Scars }\end{array}$ & Comments \\
\hline $10-20$ & No & 11.53 & 3.26 & 3 & 100 & 0 & \\
\hline $30-40$ & No & 8.81 & 5.29 & 0 & 0 & 9 & \\
\hline $30-40$ & No & 15.79 & 3.40 & 0 & 90 & 1 & \\
\hline $30-40$ & No & 9.42 & 1.75 & 1 & 0 & 2 & Rejuvination \\
\hline $40-50$ & No & 17.32 & 5.20 & 0 & 90 & 1 & \\
\hline $40-50$ & Yes & 24.06 & 3.56 & 4 & 10 & 2 & \\
\hline $40-50$ & No & 31.36 & 6.13 & 1 & 1 & 4 & Utilized \\
\hline $50-60$ & No & 16.59 & 2.01 & 0 & 0 & 1 & \\
\hline $60-70$ & Yes & 28.71 & 5.24 & 4 & 0 & 3 & \\
\hline $80-90$ & Yes & 25.73 & 2.52 & 2 & 0 & 3 & \\
\hline
\end{tabular}

The limited data available from both the shovel tests and single excavation unit indicate that the site still maintains a high degree of integrity and with additional investigations there is a high probability of finding buried, intact cultural features and discrete zones which have the potential to provide valuable information on site formation processes, subsistence, and settlement patterns.

\section{$41 \mathrm{TG} 443$}

\section{Discussion}

A noticeable rectangular depression with a rock alignment in front on the southern portion of the site suggested a possible cellar or dugout had been excavated into the shallow sediments just off the edge of the limestone shelf. A single shovel test within the depression revealed what appeared to be a wooden floor or a collapsed roof of a possible dugout structure. A single $1-\mathrm{m}^{2}$ test unit was placed near the rock alignment at what was surmised to be the entryway on the southern end of the structure (Figure 11-9). The unit was excavated to two inches below the wood layer, into sterile silty loam.

\section{Results and Analysis}

Artifacts collected from the single excavation unit include: From level 1 (0-4 inches), two clear glass sherds that probably date after the 1930s (Kendrick 1967:24; Munsey 1970:55); from Level 3 (5-6 inches), one small whiteware sherd, and a metal wire handle that may have been attached to a pail, and; from Level 4 (6-7 inches), five heavily corroded and unidentifiable metal fragments.

Based on the thickness and paste, the ceramic sherd appears to be whiteware from either a plate or saucer. The whiteware attributes along with a flowing blue pattern date its usage ca. 1880-1900 (Blake and Freeman 1998:18). Based on its context with other artifacts and within the probable dugout at the site, but without a maker's mark or more of the pattern on the sherd, this time frame is presumed (see Chapter 11). 
The research value of this site is considered high. This site may represent one of the earliest historic occupations in the San Angelo/Twin Buttes area. The presence of a dugout that may still have an intact floor is rare. The presence of flaked glass is uncommon, but appears to be a recurring theme, with flaked glass found also at 41TG437 and 41TG445. Without further testing and the recovery of diagnostic stone tools or means of absolute dating, the possibility that the lithic assemblage at the site may be historic or protohistoric cannot be ruled out. 


\section{References Cited}

Blake, M. E., and M. D. Freeman

1998 Nineteenth-century Transfer-printed Ceramics from the Texas Coast: The Quintana Collection. Prewitt and Associates, Inc. Austin, Texas.

Butler, R. F.

1992 Paleomagnetism: Magnetic Domains to Geologic Terranes. Blackwell Scientific Publications, Boston.

Kendrick, G.

1967 Bottle Fragments Betray Age of Historic Sites. El Palacio 74(2):19-24.

Munsey, C.

1970 The Illustrated Guide to Collecting Bottles. Hawthorn Books, New York.

Nickels, D. L.

2000 The Biesenbach Site (41WN88): A Case Study in Diet-Breadth. Unpublished Master's Thesis. The University of Texas at San Antonio.

Nickels, D. L., C. B. Bousman, J. D. Leach, and D. A. Cargill

1998 Test Excavations at the Culebra Creek Site, 41BX126, Bexar County, Texas. Archaeological Survey Report, No. 265. Center for Archaeological Research, The University of Texas at San Antonio; Archeology Studies Program, Report 3. Environmental Affairs Division, Texas Department of Transportation, Austin.

Stevenson, M. G.

1991 Beyond the Formation of Hearth-Associated Artifact Assemblages. In The Interpretation of Archaeological Spatial Patterning, edited by E. M. Kroll and T. D. Price, pp. 269-299. Plenum, New York.

Vierra, B. J.

1998 41MV120: A Stratified Late Archaic Site in Maverick County, Texas. Archaeological Survey Report, No. 251. Center for Archaeological Research, The University of Texas at San Antonio; Archeology Studies Program, Report 7. Environmental Affairs Division, Texas Department of Transportation, Austin. 



\section{Appendix D: Twin Buttes Archaeological Project}

\section{Surface Observation Data}

This appendix presents information on the surface observation area (SOA) procedure. As discussed in Chapter 9 , Volume 1, the goal of the SOA effort was to generate quantitative data on artifacts during the essentially noncollection survey. A series of elements were noted on each of the surface items. The attributes had to be sufficiently general such that a variety of different individuals with different backgrounds in chipped stone analysis and different levels of training could consistently record the attributes. In addition, the process had to be done quickly, maximizing the number of items recorded and the number of areas that could be investigated. Consequently, common reduction terms such as "biface thinning flake" and "preform" were avoided as the application of a specific set of criteria to several thousand flakes would have taken significant time and a substantial amount of inconsistency would certainly be introduced during the recording procedure. The attributes which were eventually selected were pared down from a more detailed list after evaluating initial tests for consistency of recording between individuals. The attributes selected included artifact types defined primarily by morphological attributes (e.g., flake, biface, uniface, retouched and/or utilized flake), maximum length and width of the artifact in $1 \mathrm{~cm}$ intervals, percentage of cortex on chipped stone items in ordinal groups (i.e., $0 \%, 1-25 \%, 26-50 \%, 51-$ $75 \%, 76-99 \%, 100 \%$ ), and material type. Forms and definitions of specific attributes used during the surface observation are presented in Appendix K.

\section{Site and Area Selection}

Sites were selected for inclusion in the sample based on both surface density and location. As our concern was generating quantitative data, those sites which were essentially defined in cutbanks or those sites noted as having few surface artifacts were generally not included in the sample. In addition, we wanted to acquire data from throughout the project area. We recorded artifacts from a total of 131 separate surface observation areas from 97 different sites. As demonstrated in Chapter 9, Figure 9-1, Volume 1, the sites blanket all areas of Twin Buttes Reservoir.

Once a site was selected as a candidate for one or more surface observation areas, a quick inspection of the surface was made with special attention to areas of differential density and exposure. Based on this inspection, one or more areas were arbitrarily selected for observation. In areas of high density, the usual procedure was to establish a circle with a three meter radius as the collection area. With lower densities, areas of various sizes and configurations were established. The boundaries of these areas were then mapped using a GPS unit. In several cases (e.g., 41TG465), the entire site was inventoried.

Once established, closely spaced transects were slowly made across the area by two to three person crews. All artifacts within a SOA were identified by the use of pin flags. Red pin flags were used for all debitage, and all tools and cores were marked with yellow pin flags. Artifacts were then recorded using the form and definitions outlined in Appendix K. Note that Dr. Raymond Mauldin recorded all tools and cores, in order to reduce the potential impact of different recorders on this category. 


\section{Table Explanation}

The following table presents information on the 131 separate SOA's. A total of 18 different columns are presented. Each of these fields is discussed below. Definitions for cores, bifaces, unifaces, tested cobbles, and utilized/retouched flakes can be found in Appendix K.

41TG\#: State of Texas archaeological trinomial number.

SOA \#: Number assigned to each SOA within the site.

Exposure (Exp.) Level: The exposure designation was made in the field by Dr. Raymond Mauldin. A designation of 1 suggests that the surface was not exposed, being characterized either by vegetation that obscured artifacts or by what appeared to be some buildup of sediment. A designation of 2 was assigned to those areas that had both little or no vegetation and evidence of some erosion. These cases frequently were shorelines consisting of extensive gravel deposits, exposed conglomerate, ridge tops with little deposition, and areas with bedrock exposed on the surface.

Tert. Flakes: Number of flakes without cortex observed within the SOA.

Secon. Flakes: Number of flakes with more than $1 \%$ and less than $50 \%$ cortex observed in the SOA.

Prim. Flakes: Number of flakes with more than $50 \%$ cortex observed in the SOA.

Cores with: Number of cores with cortex present in the SOA.

Tested Cobbles: Number of tested cobbles observed in the SOA.

Uniface with: Number of unifaces with cortex present.

Utilized with: Number of utilized or retouched flakes with cortex observed in the SOA.

Cores without: Number of cores without cortex present in the SOA.

Biface with: Number of bifaces with cortex observed in the SOA.

Utilized without: Number of utilized or retouched flakes without cortex observed in the SOA.

Biface without: Number of bifaces without cortex observed in the SOA.

Uniface without: Number of unifaces without cortex present

Metate Frag.: Number of metates, including any metate fragments, observed in the SOA.

Mano: Number of manos, including any fragments, observed in the SOA.

Hammer Stone: Number of hammer stones observed in SOA. 


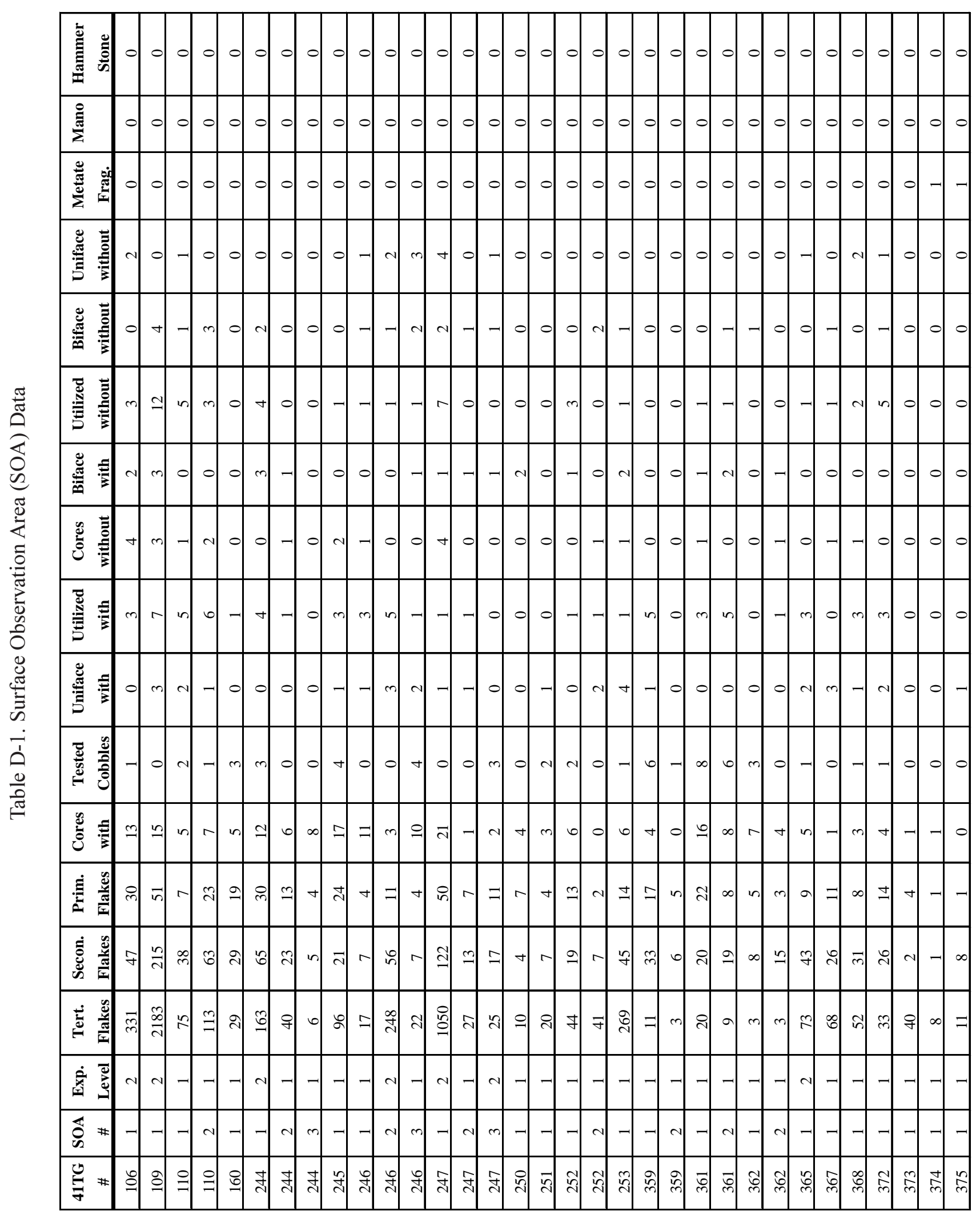




\begin{tabular}{|c|c|c|c|c|c|c|c|c|c|c|c|c|c|c|c|c|c|c|c|c|c|c|c|c|c|c|c|}
\hline 閭 & 0 & 0 & 0 & 00 & 00 & 0 & 0 & & 00 & 0 & 0 & 0 & 00 & 0 & 0 & 0 & & 0 & 0 & 0 & 0 & 00 & 0 & - & 00 & & 0 \\
\hline 离 & 0 & & 0 & 00 & 00 & 0 & o & & 00 & 0 & 0 & 0 & 00 & 0 & 0 & 0 & & 00 & 0 & 0 & of & 00 & 0 & 0 & 00 & 0 & o \\
\hline 总 & 0 & & 0 & & $=0$ & 0 & 0 & & 00 & 0 & 0 & 0 & 00 & 0 & 0 & 0 & & 0 & 0 & 0 & 0 & 00 & - & o & 00 & 0 & 0 \\
\hline 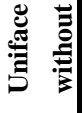 & 0 & & & & 00 & 0 & & & $0-$ & - & 0 & $m$ & 00 & 0 & 0 & of & & -10 & 0 & 0 & 0 & 00 & 0 & - & -0 & 0 & o \\
\hline 总 & - & & -10 & & $0-$ & 0 & $\sim$ & & -1 & $\nabla$ & 0 & $m$ & $\nabla$ & 6 & - & $m$ & $m \mid c$ & $\sim-$ & 0 & - & -1 & -0 & - & $N$ & $\triangle O$ & 0 & - \\
\hline 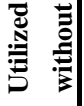 & - & & 0 & 00 & $\Rightarrow-$ & 0 & 6 & & 00 & - & 0 & - & $\sim 0$ & $\sim>$ & - & $m$ & $\neg \infty$ & $\infty$ & 0 & 0 & & $\forall \forall$ & $\infty$ & $\nabla$ & $m-$ & 0 & 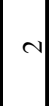 \\
\hline 总 & 0 & 0. & - & 00 & 00 & 0 & $\sim$ & 0. & -0 & 0 & 0 & - & $m$ & - & $\sim$ & 0 & $0 \mathrm{~m}$ & $m o$ & 0 & 0 & & $\curvearrowright \curvearrowright$ & - & $m$ & 00 & 0 & $m$ \\
\hline 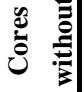 & 0 & & & 00 & 00 & 0 & 0 & $\forall$ & 00 & 0 & 0 & -1 & -0 & -1 & of & - & -0 & 00 & 0 & 0 & & 00 & $\tau$ & of & -0 & 0 & 0 \\
\hline 苞 & 0 & b. & - & 00 & $=1-$ & - & $\infty$ & $n$ & $\infty 7$ & 0 & $\mathrm{~N}$ & - & $m$ in & $a$ & - & $\sim$ & -10 & o & 0 & 0 & - & +- & - & \pm 0 & $m-$ & 0 & $\nabla$ \\
\hline 总 & 0 & of & 0 & 00 & 00 & 0 & $m$ & - & $m$ & 0 & $\sim$ & 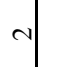 & 00 & $\sim$ & 0 & 0 & -0 & 00 & 0 & 0 & 0 & 00 & $N$ & 0 & 00 & & o \\
\hline 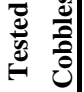 & - & & $\infty$ & & $=1+$ & -1 & $n$ & $m$ & $m-$ & of & in & & $7-$ & 0 & - & - & $0-$ & -19 & 0 & 0 & 0 & $0-1$ & $\tau$ & & $N O$ & 0 & $a$ \\
\hline 总 & - & $\infty$ & - & $\therefore c$ & $=\sigma$ & $\mathrm{A}$ & $\simeq$ & $n$ : & $9=$ & $m$ & - & $m$ & $=0$ & $=$ & $=$ & 의 & $-r$ & $-\infty$ & 0 & 0 & & $\sim \nabla$ & $\mathrm{N}$ & $\mathrm{A}$ & in - & 0 & $\pi$ \\
\hline 赔 & in & $=$ & ef & $n$ in & $n$ & $=$ & $\stackrel{\sim}{\searrow}$ & 9 & $m=$ & $\sigma$ & $\bar{m}$ & 의 & $\stackrel{\infty}{m} \overbrace{}^{\infty}$ & त & 9 & ¿ & त & : $\left.{ }^{\infty}\right)$ & 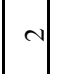 & $\stackrel{-}{-}$ & $\infty:$ & $\therefore=$ & \pm & 잉 & तो - & & en \\
\hline 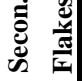 & $\infty$ & 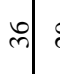 & $\overrightarrow{2}$ & $\therefore$ & $\theta$ iे & - & $\bar{n}$ & 인 & $\exists v$ & $m$ & ले & $m$ & ले $\forall$ & 8 & $q$ & in & n & 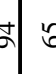 & $\infty$ & $\stackrel{\infty}{f}$ & $=9$ & ले & $\bar{\lambda}$ & $\begin{array}{lll} \pm & 0\end{array}$ & 卆o & & 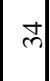 \\
\hline 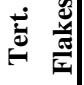 & 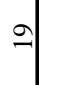 & $n$ & N & $\infty \leq$ & $=\stackrel{m}{m}$ & $\infty$ & 의 & की & तิ & $n$ & ते & त) & ते ते & in & $\stackrel{\infty}{\sim} \mid$ & ल & ป & ثे & $\approx$ & 帘 & 의 & 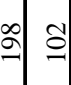 & $\widehat{\sigma}$ & $\vec{n}$ & 6ी 0 & & הి \\
\hline 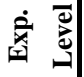 & $\ldots$ & & $\mathrm{N}$ & & $\mathrm{N}$ & -1 & -1 & & - & $N$ & - & - & -0 & $\curvearrowright$ & - & - & $\sim$ & $N-$ & $a$ & $N$ & & $\sim-$ & - & & -- & - & - \\
\hline$\underset{\mathscr{D}}{\mathbb{2}} \#$ & - & $N$ & - & $\sim$ & n & - & - & $\sim$ & -0 & - & - & - & $-\tau$ & - & - & $\sim$ & $-\alpha$ & $N-$ & - & $\mathrm{N}$ & & $\sim-$ & $\sim$ & - & -- & - & - \\
\hline$\underset{f}{=} \#$ & $\stackrel{\infty}{m}$ & $\stackrel{\infty}{m}$ & 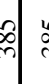 & 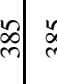 & $\begin{array}{c}n \\
0\end{array}$ & $\mid \begin{array}{l}0 \\
0 \\
0\end{array}$ & $\begin{array}{l}\infty \\
\infty \\
m\end{array}$ & \begin{tabular}{c|c}
$\infty$ \\
$m$ \\
$m$
\end{tabular} & $\begin{array}{l}\dot{m}^{\infty} \\
m\end{array}$ & के & ले & ले & 今े & 원 & | & tे & 尚 & :ी & oे & oे & $\exists$ & $\exists \quad \frac{7}{7}$ & $\frac{7}{7}$ & $\vec{\nabla}$ & ने & $\bar{\gamma}$ & ปิ \\
\hline
\end{tabular}




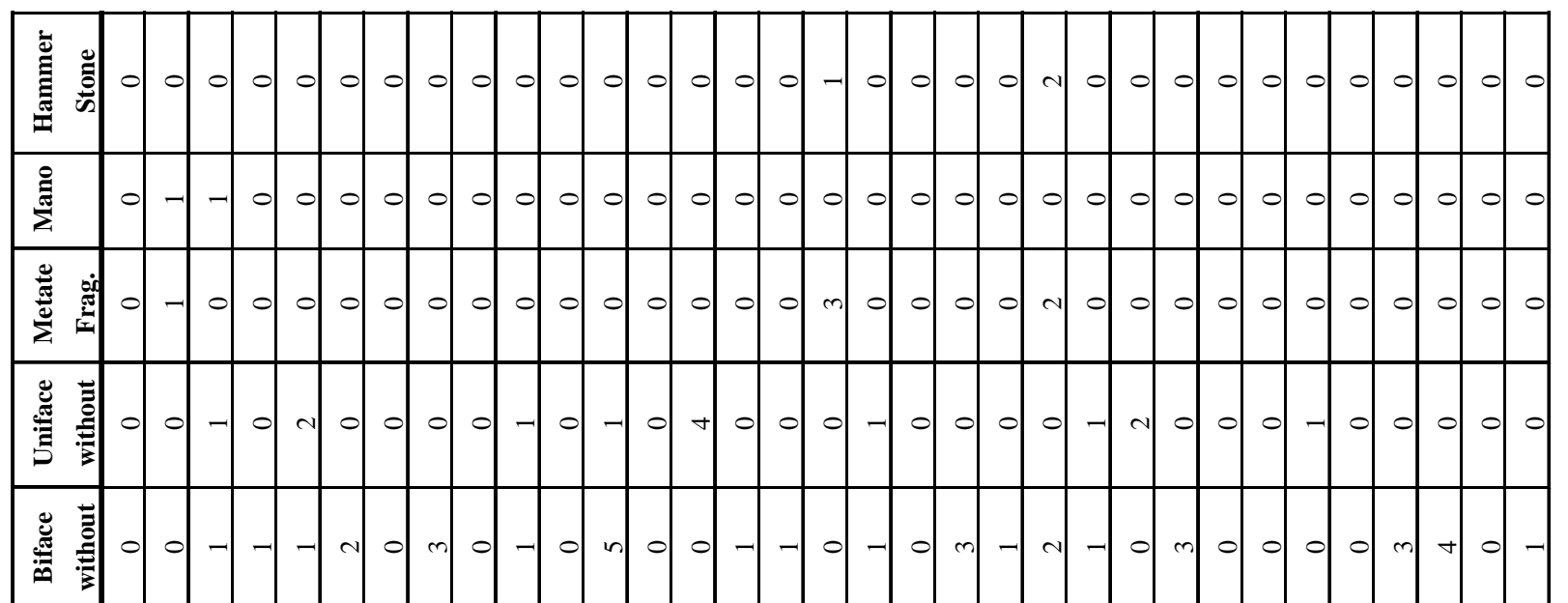

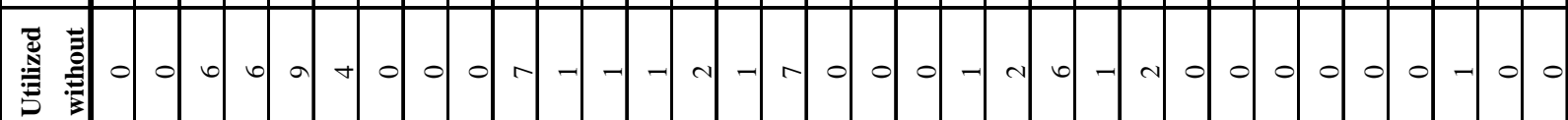

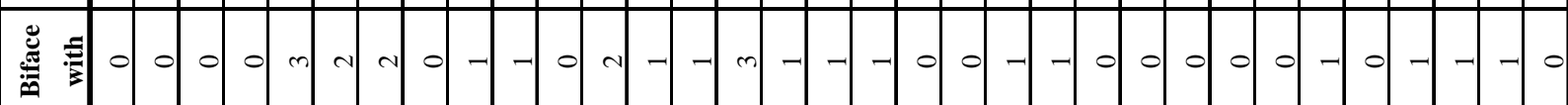

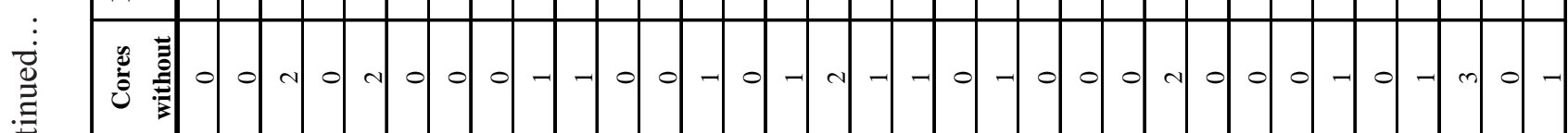

焉 部。

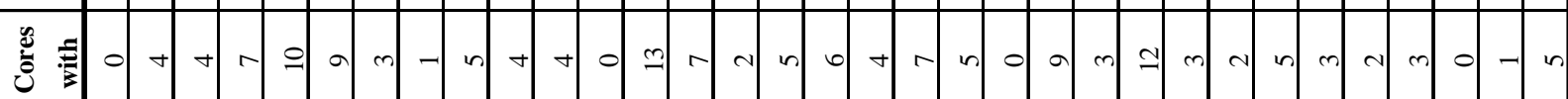

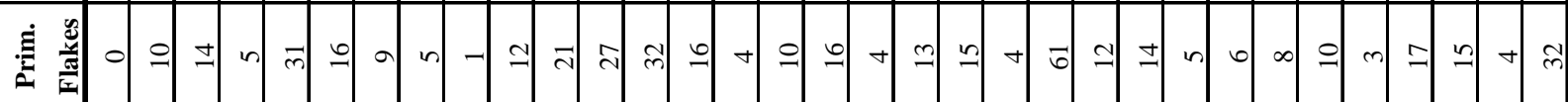

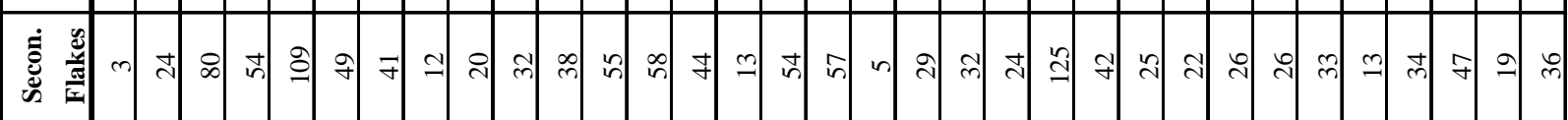

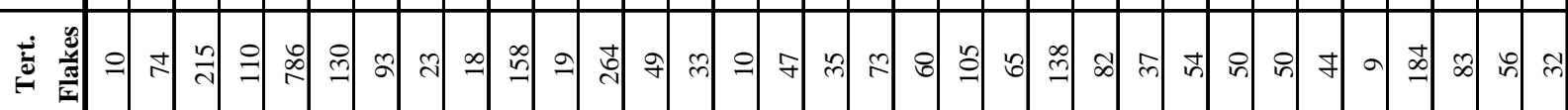

离

遇\#-cm

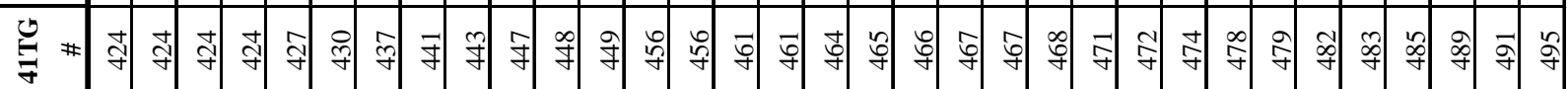




\begin{tabular}{|c|c|c|c|c|c|c|c|c|c|c|c|c|c|c|c|c|c|c|c|c|c|c|c|c|c|c|c|c|c|}
\hline 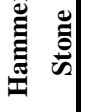 & 0 & 0 & 0 & 0 & & & 0 & of & 0 & 0 & & of & 0 & 00 & 00 & 0 & 0 & of & 0 & 0 & 00 & 0 & 0 & 00 & 0 & 0 & 0 & 0 & 0 \\
\hline 密 & of & 0 & 0 & 0 & & 0 & 0 & of & 0 & 0 & 0 & of & 0 & & $\begin{array}{ll}0 & 0\end{array}$ & 0 & 0 & 0 & o & 0 & 00 & 0 & 0 & 00 & 00 & 0 & 0 & 0 & of \\
\hline 密 & 0 & o & 0 & 0 & 0 & 0 & 0 & 0 & 0 & 0 & 0 & 0 & 0 & & 00 & 0 & 0 & 0 & of & 0 & 00 & 0 & 0 & 00 & 00 & 0 & 0 & 0 & of \\
\hline 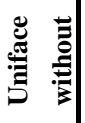 & 0 & o & 0 & - & & 0 & 0 & - & 0 & 0 & 0 & 0 & - & 0 & -0 & -1 & - & - & 0 & 0 & $\begin{array}{lll}0 & 0\end{array}$ & - & 0 & $\sim-$ & -1 & 0 & 0 & 0 & o \\
\hline 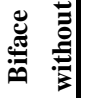 & - & o & - & 0 & $\checkmark$ & 0 & - & in & 0 & - & 0 & 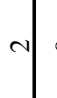 & 0 & & 00 & - & 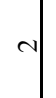 & $\sim$ & o & 0 & \begin{tabular}{l|l|l|}
0 & 4
\end{tabular} & -1 & - & $m 0$ & $=-1$ & - & in & $\theta$ & $c$ \\
\hline 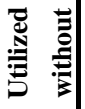 & & 0 & 0 & 0 & & of & $N$ & - & of & o & 0 & o & & 01 & -- & • & $\approx$ & $m$ & 0 & $\sim$ & $\begin{array}{lll}a \\
y\end{array}$ & -1 & 0 & -- & $-1-1$ & - & & $\nabla$ & - \\
\hline 总 & $N$ & 0 & 0 & 0 & 0 & o & 0 & $\forall$ & - & 0 & 0 & - & $\triangle$ & 00 & $0 \mathrm{~m}$ & - & 0 & of & 0 & 0 & $\begin{array}{lll}0 & 0\end{array}$ & 10 & 0 & 00 & 00 & 0 & m & - & 0 \\
\hline 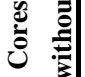 & 0 & o & - & 0 & 0 & - & 0 & $\sim$ & 0 & 0 & 0 & 0 & $m$ & -0 & 00 & - & $m$ & 0 & 0 & 0 & 00 & 0 & - & -0 & 0 & 0 & - & - & r \\
\hline 汽 & $\sim$ & - & - & - & $m$ & 0 & 0 & $m$ & - & 0 & 0 & 0 & - & 0. & $\triangle 0$ & $\nabla$ & $\infty$ & $\sim$ & 0 & $\sim$ & 00 & 0 & - & $n-$ & $-\lambda$ & $m$ & $m$ & $\nabla$ & 7 \\
\hline 莺 部 & 0 & & 0 & 0 & & 0 & 0 & of & of & 0 & o & 0 & $\mathrm{~N}$ & 00 & 00 & - & 0 & -1 & 0 & 0. & -0 & - & 0 & -0 & $=0$ & 0 & & - & 0 \\
\hline 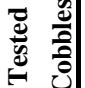 & 7 & - & - & 0 & 0 & of & o & of & in & $\sim$ & o & o & 0 & 00 & $0 \mathrm{~m}$ & 0 & - & o & 0 & 0. & -1 & $\nabla$ & 0 & -0 & 0 & $m$ & & 0 & - \\
\hline 总 & - & 0 & $\nabla$ & - & $N$ & N & 0 & $\sim$ & $\nabla$ & 0 & 0 & $m$ & $\exists$ & 4 & n 0 & $N \mid$ & $\nabla$ & o & in & $\sim 0$ & -1 & 의 & 0 & o - & $-m$ & $a$ & 7 & $\checkmark$ & $\sim$ \\
\hline 焉 & $=$ & - & $\infty$ & ? & $\ddot{\imath}$ & $=$ & $\lambda$ & ते & in & o & ๑) & c) & a & & $\nabla \stackrel{-}{-}$ & 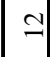 & 2 & 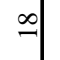 & $\sigma$ & & $n \pm$ & $=$ & in & $\infty$ & -0 & $\because$ & तิ & $\simeq$ & $y$ \\
\hline 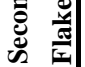 & $m$ & $=$ & $\exists$ & ก) & ले & मे & $\simeq$ & $\vec{~}$ & $\cong$ & $m$ & $\nabla$ & $\infty$ & n $=$ & & $\sigma \stackrel{m}{~}$ & $\infty$ & $\approx$ & 8 & $\nabla$ & $=\mathrm{s}$ & $\forall \approx$ & $=$ & $\cong$ & 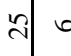 & 0 o & 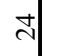 & & $\stackrel{\infty}{q}$ & 근 \\
\hline 这 & $\exists$ & 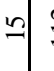 & $\exists$ & 8 & ๙ & है। & $\stackrel{\infty}{\infty}$ & $\underline{n}$ & \pm & $r$ & a & is & $\bar{m}$ & of & $\therefore$ in & 의 & 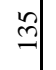 & : & $\nabla$ & $+\delta$ & 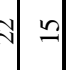 & \pm & ה & $\stackrel{\infty}{+}$ & $\circ=$ & के & & I & in \\
\hline 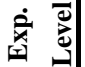 & - & - & - & - & - & $\sim$ & N & - & -1 & - & & - & - & -1 & -4 & 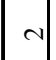 & 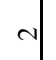 & $\sim$ & - & $-c$ & 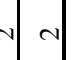 & - & - & $-c$ & $4-$ & - & - & - & - \\
\hline$\underset{\delta}{\mathbb{S}} \#$ & - & - & - & $\sim$ & $m$ & - & $\sim$ & -1 & - & - & - & - & -1 & -- & -- & - & $\sim$ & - & - & - & $-A$ & - & - & -- & $-1-1$ & - & - & - & - \\
\hline$\underset{F}{b}=$ & ఫे & ât & ㅇำ & ㅇํํ| & 인 & 常 & 苟 & : & ही & $\stackrel{\infty}{i}$ & ฉे & ज् & n. & $m$ & $\vec{n} \mid \frac{m}{n}$ & $\stackrel{\infty}{n}$ & $\stackrel{\infty}{n}$ & iे & तิ & กี & กิ & in & nี & in & הत) & in & $\bar{m}$ & กิ & ڤి \\
\hline
\end{tabular}




\section{Appendix E: Twin Buttes Archaeological Project}

\section{Data on all Bifaces Observed and/or Collected}

Table E-1 provides data on 294 bifaces observed and/or collected on the Twin Buttes survey. Ten different columns are present.

41TG\#: State of Texas archaeological site trinomial. Isolated items are designated as 0.

Complete Length: 1 = length measurement is complete; $0=$ length measurement is partial.

Length cm.: Maximum length in $\mathrm{cm}$.

Complete Width: $1=$ width measurement is complete; $0=$ width measurement is partial.

Width cm.: Maximum width in $\mathrm{cm}$.

Complete Thickness: 1 = thickness measurement is complete; $0=$ thickness measurement is partial.

Thickness cm.: Maximum thickness in $\mathrm{cm}$.

Biface Shape: $0=$ irregular; $1=$ round to oval in outline; $3=$ diamond shaped; $4=$ leaf shaped; $5=$ other; $8=$ projectile point.

Cortex Present: $0=$ no cortex; $1=$ less than $50 \%$ cortex cover; $2=$ more than $50 \%$ cortex cover.

Material Types: The vast majority of the material was coded as 1 - which is either gray, brown, or blue-gray. Codes 2 and 4 are white and/or pale white. Code 3 is either pinkish or red; often heat-treated. Code 9 is any other color. 
Table E-1. Data on Bifaces Observed and/or Collected During the Twin Buttes Survey

\begin{tabular}{|c|c|c|c|c|c|c|c|c|c|}
\hline $\begin{array}{c}\text { 41TG } \\
\#\end{array}$ & \begin{tabular}{|c|}
$\begin{array}{c}\text { Complete } \\
\text { Length }\end{array}$ \\
\end{tabular} & $\begin{array}{c}\text { Length } \\
\mathrm{cm} .\end{array}$ & $\begin{array}{c}\text { Complete } \\
\text { Width }\end{array}$ & $\begin{array}{l}\text { Width } \\
\text { cm. }\end{array}$ & $\begin{array}{l}\text { Complete } \\
\text { Thickness }\end{array}$ & \begin{tabular}{|c} 
Thickness \\
cm.
\end{tabular} & $\begin{array}{l}\text { Biface } \\
\text { Shape }\end{array}$ & $\begin{array}{c}\text { Cortex } \\
\text { Present } \\
\end{array}$ & $\begin{array}{c}\text { Materia } \\
\text { Types }\end{array}$ \\
\hline 0 & 0 & 3.111 & 0 & 3.788 & 1 & 1.022 & 0 & 0 & 2 \\
\hline 0 & 1 & 8.667 & 1 & 5.891 & 1 & 1.233 & 0 & 1 & 1 \\
\hline 0 & 1 & 4.010 & 0 & 2.730 & 1 & .669 & 3 & 0 & 1 \\
\hline 0 & 1 & 4.726 & 1 & 2.308 & 1 & .722 & 4 & 0 & 1 \\
\hline 0 & 0 & 3.522 & 0 & 2.175 & 1 & .648 & 8 & 0 & 1 \\
\hline 0 & 1 & 4.229 & 0 & 2.998 & 1 & .633 & 8 & 0 & 9 \\
\hline 0 & 1 & 4.710 & 1 & 3.244 & 1 & .617 & 8 & 0 & 9 \\
\hline 0 & 0 & 4.744 & 1 & 3.683 & 1 & 1.023 & 8 & 0 & 9 \\
\hline 0 & 1 & 5.578 & 1 & 3.061 & 1 & .748 & 8 & 0 & 9 \\
\hline 0 & 0 & 4.518 & 1 & 4.304 & 1 & .760 & 8 & 0 & 1 \\
\hline 106 & 1 & 6.010 & 1 & 3.820 & 1 & 1.910 & 4 & 1 & 1 \\
\hline 106 & 1 & 6.460 & 1 & 4.150 & 1 & 1.540 & 4 & 2 & 1 \\
\hline 109 & 0 & 3.350 & 1 & 3.860 & 1 & .700 & 0 & 0 & 9 \\
\hline 109 & 0 & 3.930 & 1 & 3.970 & 1 & 1.700 & 0 & 0 & 1 \\
\hline 109 & 1 & 4.950 & 1 & 3.510 & 1 & .820 & 1 & 1 & 3 \\
\hline 109 & 1 & 5.980 & 1 & 4.380 & 1 & 1.270 & 1 & 1 & 1 \\
\hline 109 & 1 & 7.140 & 1 & 3.980 & 1 & 1.790 & 4 & 1 & 1 \\
\hline 109 & 1 & 2.640 & 1 & 2.000 & 1 & .610 & 8 & 0 & 3 \\
\hline 110 & 0 & 3.013 & 0 & 2.360 & 0 & .560 & 0 & 0 & 1 \\
\hline 110 & 0 & 3.040 & 0 & 6.470 & 1 & 2.900 & 0 & 0 & 1 \\
\hline 110 & 0 & 4.750 & 1 & 4.030 & 1 & 1.860 & 0 & 0 & 1 \\
\hline 110 & 1 & 2.960 & 1 & 1.290 & 1 & .380 & 8 & 0 & 9 \\
\hline 244 & 0 & 4.060 & 1 & 3.030 & 1 & .720 & 0 & 0 & 1 \\
\hline 244 & 1 & 6.310 & 1 & 3.900 & 1 & 1.090 & 1 & 2 & 1 \\
\hline 244 & 1 & 6.926 & 1 & 4.135 & 1 & 2.078 & 4 & 0 & 9 \\
\hline 244 & 1 & 2.890 & 1 & 1.620 & 1 & .030 & 8 & 0 & 1 \\
\hline 244 & 1 & 12.210 & 1 & 4.830 & 1 & 2.530 & 4 & 1 & 1 \\
\hline 246 & 0 & 2.060 & 1 & 2.810 & 1 & .590 & 0 & 0 & 1 \\
\hline 246 & 0 & 2.560 & 1 & 2.960 & 1 & .720 & 0 & 0 & 1 \\
\hline 246 & 0 & 4.050 & 1 & 2.180 & 1 & 1.080 & 0 & 0 & 1 \\
\hline 246 & 1 & 7.880 & 1 & 4.160 & 1 & 1.640 & 4 & 0 & 1 \\
\hline 246 & 0 & 2.981 & 1 & 2.179 & 1 & .610 & 8 & 0 & 1 \\
\hline 247 & 0 & 8.390 & 0 & 6.100 & 0 & 1.500 & 0 & 1 & 1 \\
\hline 247 & 0 & 1.990 & 0 & 1.905 & 1 & .582 & 8 & 0 & 1 \\
\hline 247 & 1 & 3.760 & 1 & 2.576 & 1 & .784 & 8 & 0 & 1 \\
\hline 247 & 0 & 4.190 & 1 & 3.250 & 1 & .970 & 0 & 0 & 1 \\
\hline 247 & 1 & 6.910 & 1 & 4.620 & 1 & 2.610 & 1 & 2 & 1 \\
\hline 247 & 0 & 7.810 & 1 & 5.250 & 1 & 1.380 & 0 & 1 & 1 \\
\hline 247 & 1 & 4.250 & 1 & 3.150 & 1 & .760 & 5 & 0 & 1 \\
\hline 250 & 1 & 7.190 & 1 & 4.190 & 1 & 1.360 & 4 & 1 & 1 \\
\hline 250 & 1 & 9.120 & 1 & 4.660 & 1 & 1.160 & 4 & 1 & 1 \\
\hline 252 & 0 & 2.646 & 1 & 2.067 & 1 & .544 & 5 & 0 & 1 \\
\hline 252 & 0 & 3.977 & 1 & 2.585 & 1 & .604 & 8 & 0 & 1 \\
\hline 252 & 1 & 5.250 & 1 & 3.460 & 1 & 1.020 & 4 & 0 & 1 \\
\hline 252 & 1 & 6.060 & 1 & 3.510 & 1 & .710 & 4 & 0 & 1 \\
\hline 252 & 1 & 6.150 & 1 & 4.590 & 1 & 2.110 & 5 & 1 & 9 \\
\hline 253 & 0 & 2.460 & 1 & 2.580 & 1 & .630 & 0 & 0 & 4 \\
\hline 253 & 1 & 5.280 & 1 & 4.790 & 1 & 1.490 & 1 & 1 & 1 \\
\hline 253 & 1 & 6.100 & 1 & 4.250 & 1 & 1.770 & 1 & 1 & 1 \\
\hline 253 & 1 & 4.824 & 1 & 2.599 & 1 & .659 & 8 & 0 & 1 \\
\hline 362 & 0 & 4.440 & 1 & 3.440 & 1 & .664 & 0 & 0 & 1 \\
\hline
\end{tabular}


Table E-1. continued...

\begin{tabular}{|c|c|c|c|c|c|c|c|c|c|}
\hline $\begin{array}{c}11 \mathrm{TG} \\
\#\end{array}$ & \begin{tabular}{|c|}
$\begin{array}{c}\text { Complete } \\
\text { Length }\end{array}$ \\
\end{tabular} & $\begin{array}{l}\text { Length } \\
\mathrm{cm} .\end{array}$ & $\begin{array}{c}\begin{array}{c}\text { Complete } \\
\text { Width }\end{array} \\
\end{array}$ & $\begin{array}{l}\text { Width } \\
\mathrm{cm} .\end{array}$ & $\begin{array}{l}\text { Complete } \\
\text { Thickness } \\
\end{array}$ & \begin{tabular}{|c}
$\begin{array}{c}\text { Thickness } \\
\text { cm. }\end{array}$ \\
\end{tabular} & $\begin{array}{l}\text { Biface } \\
\text { Shape }\end{array}$ & $\begin{array}{c}\text { Cortex } \\
\text { Present } \\
\end{array}$ & $\begin{array}{c}\text { Material } \\
\text { Types }\end{array}$ \\
\hline 362 & 0 & 2.393 & 0 & 2.235 & 1 & .783 & 8 & 0 & 1 \\
\hline 364 & 0 & 3.197 & 1 & 3.661 & 1 & .723 & 8 & 0 & 1 \\
\hline 365 & 0 & 3.570 & 1 & 3.230 & 1 & .712 & 8 & 0 & 1 \\
\hline 367 & 0 & 2.800 & 1 & 2.810 & 1 & .640 & 0 & 0 & 1 \\
\hline 367 & 0 & 1.550 & 0 & 1.870 & 1 & .504 & 8 & 0 & 9 \\
\hline 367 & 0 & 1.976 & 0 & 2.501 & 0 & .485 & 8 & 0 & 1 \\
\hline 367 & 0 & 3.130 & 1 & 3.610 & 1 & .645 & 8 & 0 & 1 \\
\hline 369 & 0 & 3.170 & 0 & 1.713 & 1 & .377 & 0 & 0 & 1 \\
\hline 370 & 0 & 4.490 & 1 & 2.320 & 1 & .834 & 0 & 0 & 1 \\
\hline 372 & 0 & 3.250 & 1 & 7.480 & 1 & 2.360 & 0 & 0 & 1 \\
\hline 373 & 0 & 3.996 & 0 & 2.995 & 1 & .636 & 0 & 0 & 1 \\
\hline 378 & 0 & 4.636 & 1 & 3.514 & 1 & .806 & 0 & 0 & 1 \\
\hline 378 & 0 & 5.110 & 1 & 3.490 & 1 & 1.150 & 0 & 0 & 1 \\
\hline 378 & 0 & 4.373 & 1 & 2.442 & 1 & .744 & 5 & 1 & 1 \\
\hline 378 & 0 & 3.409 & 1 & 2.559 & 1 & .734 & 8 & 0 & 1 \\
\hline 381 & 1 & 5.063 & 1 & 2.729 & 1 & .711 & 8 & 0 & 1 \\
\hline 382 & 1 & 5.626 & 1 & 2.703 & 1 & .835 & 1 & 0 & 1 \\
\hline 382 & 1 & 7.047 & 1 & 3.211 & 1 & .659 & 8 & 0 & 1 \\
\hline 385 & 0 & 3.660 & 1 & 5.230 & 1 & 1.960 & 0 & 0 & 1 \\
\hline 385 & 0 & 4.690 & 1 & 4.760 & 1 & 2.020 & 0 & 1 & 1 \\
\hline 385 & 1 & 6.300 & 1 & 2.970 & 1 & 1.650 & 4 & 0 & 1 \\
\hline 385 & 1 & 6.550 & 1 & 5.090 & 1 & 1.090 & 4 & 0 & 1 \\
\hline 388 & 0 & 4.020 & 1 & 4.920 & 1 & .990 & 0 & 0 & 1 \\
\hline 388 & 0 & 7.500 & 1 & 6.020 & 1 & 1.910 & 0 & 0 & 1 \\
\hline 388 & 0 & 7.500 & 1 & 7.550 & 1 & 2.250 & 0 & 1 & 1 \\
\hline 388 & 1 & 5.920 & 1 & 6.020 & 1 & 2.290 & 1 & 0 & 1 \\
\hline 388 & 1 & 9.250 & 1 & 8.340 & 1 & 3.420 & 1 & 1 & 4 \\
\hline 388 & 1 & 4.542 & 1 & 3.072 & 1 & .505 & 8 & 0 & 1 \\
\hline 389 & 0 & 5.700 & 1 & 3.990 & 1 & 1.140 & 0 & 0 & 1 \\
\hline 389 & 1 & 7.890 & 1 & 4.900 & 1 & 1.880 & 1 & 1 & 1 \\
\hline 389 & 0 & .862 & 0 & 1.521 & 1 & .386 & 8 & 0 & 1 \\
\hline 389 & 0 & 3.530 & 0 & 2.482 & 1 & .557 & 8 & 0 & 1 \\
\hline 390 & 0 & 3.543 & 1 & 3.031 & 1 & .417 & 8 & 0 & 1 \\
\hline 391 & 0 & 3.210 & 1 & 3.110 & 1 & 1.010 & 0 & 0 & 1 \\
\hline 391 & 0 & 5.100 & 1 & 3.250 & 1 & .690 & 0 & 0 & 1 \\
\hline 391 & 1 & 7.480 & 1 & 3.790 & 1 & 1.070 & 4 & 0 & 9 \\
\hline 391 & 0 & 3.371 & 1 & 2.569 & 1 & .609 & 8 & 0 & 1 \\
\hline 395 & 1 & 9.320 & 1 & 8.410 & 1 & 2.580 & 1 & 0 & 1 \\
\hline 395 & 1 & 8.190 & 1 & 6.220 & 1 & 2.520 & 4 & 0 & 9 \\
\hline 395 & 1 & 11.640 & 1 & 7.800 & 1 & 2.430 & 4 & 1 & 1 \\
\hline 395 & 0 & .830 & 0 & .930 & 1 & .120 & 8 & 0 & 9 \\
\hline 397 & 0 & 4.000 & 1 & 6.300 & 1 & 1.890 & 0 & 0 & 1 \\
\hline 397 & 0 & 4.440 & 1 & 4.690 & 1 & 1.950 & 0 & 1 & 1 \\
\hline 397 & 0 & 4.930 & 1 & 8.310 & 1 & 1.860 & 0 & 1 & 2 \\
\hline 397 & 0 & 5.430 & 1 & 4.130 & 1 & 1.640 & 0 & 0 & 1 \\
\hline 397 & 0 & 6.930 & 0 & 4.980 & 1 & 2.290 & 0 & 0 & 1 \\
\hline 397 & 1 & 12.660 & 1 & 10.210 & 1 & 1.960 & 0 & 0 & 4 \\
\hline 397 & 1 & 13.260 & 1 & 9.360 & 1 & 2.350 & 1 & 1 & 1 \\
\hline 397 & 1 & 7.350 & 1 & 5.220 & 1 & 2.660 & 4 & 1 & 1 \\
\hline 397 & 1 & 9.920 & 1 & 4.530 & 1 & 2.360 & 4 & 1 & 1 \\
\hline 400 & 0 & 2.770 & 0 & 5.640 & 1 & 1.810 & 0 & 0 & 1 \\
\hline
\end{tabular}


Table E-1. continued...

\begin{tabular}{|c|c|c|c|c|c|c|c|c|c|}
\hline $\begin{array}{c}\text { 41TG } \\
\#\end{array}$ & $\begin{array}{c}\text { Complete } \\
\text { Length }\end{array}$ & $\begin{array}{c}\text { Length } \\
\mathrm{cm} .\end{array}$ & $\begin{array}{c}\text { Complete } \\
\text { Width }\end{array}$ & $\begin{array}{c}\text { Width } \\
\mathrm{cm} .\end{array}$ & $\begin{array}{l}\text { Complete } \\
\text { Thickness }\end{array}$ & $\begin{array}{c}\text { Thickness } \\
\mathrm{cm} .\end{array}$ & $\begin{array}{l}\text { Biface } \\
\text { Shape }\end{array}$ & $\begin{array}{c}\text { Cortex } \\
\text { Present } \\
\end{array}$ & $\begin{array}{c}\text { Materia } \\
\text { Types } \\
\end{array}$ \\
\hline 400 & 0 & 3.170 & 1 & 3.370 & 1 & .735 & 0 & 0 & 1 \\
\hline 400 & 1 & 5.350 & 1 & 4.220 & 1 & 1.400 & 1 & 0 & 1 \\
\hline 400 & 1 & 6.840 & 1 & 3.310 & 1 & 1.800 & 1 & 0 & 2 \\
\hline 400 & 1 & 3.090 & 1 & 1.800 & 1 & .710 & 4 & 0 & 1 \\
\hline 400 & 1 & 5.740 & 1 & 3.110 & 1 & 1.490 & 4 & 0 & 1 \\
\hline 400 & 1 & 11.140 & 1 & 4.970 & 1 & 2.820 & 4 & 1 & 1 \\
\hline 404 & 1 & 5.760 & 1 & 3.010 & 1 & 1.780 & 0 & 0 & 1 \\
\hline 404 & 1 & 5.560 & 1 & 4.690 & 1 & 2.950 & 1 & 1 & 1 \\
\hline 404 & 1 & 8.710 & 1 & 5.350 & 1 & 2.790 & 1 & 0 & 9 \\
\hline 404 & 1 & 8.820 & 1 & 6.780 & 1 & 2.730 & 1 & 1 & 1 \\
\hline 404 & 1 & 4.850 & 1 & 3.810 & 1 & .880 & 4 & 0 & 2 \\
\hline 404 & 1 & 9.550 & 1 & 4.830 & 1 & 2.230 & 4 & 0 & 1 \\
\hline 404 & 1 & 4.077 & 1 & 2.317 & 1 & .554 & 8 & 0 & 1 \\
\hline 405 & 0 & 1.750 & 0 & 2.520 & 1 & .710 & 0 & 0 & 1 \\
\hline 405 & 0 & 3.510 & 1 & 2.910 & 1 & .580 & 0 & 0 & 1 \\
\hline 405 & 0 & 3.670 & 0 & 3.810 & 1 & .900 & 0 & 0 & 1 \\
\hline 405 & 1 & 5.250 & 1 & 3.410 & 1 & 2.050 & 1 & 1 & 1 \\
\hline 405 & 1 & 8.240 & 1 & 5.850 & 1 & 2.290 & 1 & 1 & 9 \\
\hline 405 & 1 & 5.690 & 1 & 4.080 & 1 & 1.990 & 4 & 0 & 1 \\
\hline 405 & 1 & 8.360 & 1 & 7.110 & 1 & 1.900 & 5 & 1 & 1 \\
\hline 405 & 0 & 1.341 & 0 & 1.836 & 1 & .750 & 8 & 0 & 1 \\
\hline 405 & 0 & 2.400 & 1 & 2.510 & 1 & .250 & 8 & 0 & 1 \\
\hline 408 & 0 & 3.330 & 1 & 2.400 & 1 & .380 & 0 & 0 & 1 \\
\hline 409 & 0 & 1.300 & 0 & 1.990 & 1 & .640 & 8 & 0 & 1 \\
\hline 410 & 1 & 5.043 & 1 & 3.206 & 1 & .548 & 8 & 0 & 1 \\
\hline 411 & 0 & 1.980 & 1 & 2.110 & 1 & .270 & 0 & 0 & 1 \\
\hline 411 & 0 & 3.870 & 1 & 4.520 & 1 & 1.250 & 0 & 1 & 1 \\
\hline 411 & 0 & 4.170 & 1 & 4.700 & 1 & 2.330 & 0 & 1 & 1 \\
\hline 411 & 0 & 7.850 & 1 & 4.330 & 1 & 2.390 & 4 & 1 & 1 \\
\hline 412 & 0 & 5.240 & 1 & 5.380 & 1 & 2.060 & 0 & 1 & 1 \\
\hline 412 & 0 & 5.710 & 1 & 4.160 & 1 & 1.090 & 1 & 1 & 1 \\
\hline 412 & 1 & 6.350 & 1 & 3.890 & 1 & 1.620 & 1 & 1 & 1 \\
\hline 412 & 1 & 4.210 & 1 & 2.880 & 1 & 1.330 & 4 & 0 & 1 \\
\hline 412 & 0 & 2.760 & 1 & 2.876 & 1 & .713 & 8 & 0 & 1 \\
\hline 412 & 0 & 3.072 & 1 & 2.609 & 1 & .707 & 8 & 0 & 1 \\
\hline 412 & 0 & 3.097 & 1 & 2.851 & 1 & .643 & 8 & 0 & 1 \\
\hline 413 & 0 & 3.458 & 1 & 2.399 & 1 & .755 & 8 & 0 & 1 \\
\hline 414 & 0 & 3.370 & 1 & 2.710 & 1 & 1.120 & 0 & 1 & 1 \\
\hline 414 & 0 & 4.480 & 0 & 3.310 & 1 & 1.650 & 0 & 2 & 2 \\
\hline 418 & 0 & 3.270 & 1 & 2.947 & 1 & .646 & 8 & 0 & 1 \\
\hline 419 & 1 & 7.100 & 1 & 5.370 & 1 & 1.100 & 0 & 0 & 1 \\
\hline 419 & 0 & 6.400 & 1 & 5.830 & 1 & 2.780 & 1 & 0 & 9 \\
\hline 423 & 0 & 5.960 & 1 & 4.370 & 1 & 2.250 & 0 & 1 & 1 \\
\hline 423 & 1 & 6.840 & 1 & 5.310 & 1 & 1.170 & 4 & 1 & 1 \\
\hline 423 & 1 & 7.660 & 1 & 4.890 & 1 & 3.970 & 4 & 0 & 1 \\
\hline 423 & 1 & 8.230 & 1 & 5.220 & 1 & 2.050 & 4 & 2 & 1 \\
\hline 424 & 0 & 3.870 & 1 & 2.900 & 1 & .700 & 0 & 0 & 1 \\
\hline 424 & 0 & 1.210 & 1 & .590 & 1 & .180 & 8 & 0 & 1 \\
\hline 424 & 0 & 2.809 & 0 & 1.296 & 1 & .020 & 8 & 0 & 1 \\
\hline 424 & 1 & 4.690 & 1 & 4.065 & 1 & .681 & 8 & 0 & 1 \\
\hline 424 & 0 & 5.899 & 1 & 3.562 & 1 & .850 & 8 & 0 & 1 \\
\hline
\end{tabular}


Table E-1. continued...

\begin{tabular}{|c|c|c|c|c|c|c|c|c|c|}
\hline $\begin{array}{c}41 \mathrm{TG} \\
\# \\
\end{array}$ & \begin{tabular}{|c|}
$\begin{array}{c}\text { Complete } \\
\text { Length }\end{array}$ \\
\end{tabular} & $\begin{array}{l}\text { Length } \\
\mathrm{cm} .\end{array}$ & $\begin{array}{c}\text { Complete } \\
\text { Width }\end{array}$ & $\begin{array}{c}\text { Width } \\
\mathrm{cm} .\end{array}$ & \begin{tabular}{|l} 
Complete \\
Thickness \\
\end{tabular} & \begin{tabular}{|c|c|} 
Thickness \\
$\mathbf{c m}$.
\end{tabular} & $\begin{array}{l}\text { Biface } \\
\text { Shape }\end{array}$ & $\begin{array}{c}\text { Cortex } \\
\text { Present } \\
\end{array}$ & $\begin{array}{c}\text { Material } \\
\text { Types } \\
\end{array}$ \\
\hline 427 & 0 & 3.230 & 1 & 4.210 & 1 & 1.070 & 0 & 1 & 1 \\
\hline 427 & 0 & 6.290 & 1 & 3.840 & 1 & 1.280 & 0 & 1 & 1 \\
\hline 427 & 1 & 6.220 & 1 & 5.240 & 1 & 2.260 & 1 & 1 & 1 \\
\hline 427 & 1 & 7.270 & 1 & 5.880 & 1 & 2.230 & 1 & 0 & 1 \\
\hline 427 & 0 & 2.620 & 1 & 1.860 & 1 & .458 & 8 & 0 & 9 \\
\hline 427 & 0 & 2.686 & 1 & 2.327 & 1 & .642 & 8 & 0 & 1 \\
\hline 427 & 1 & 3.310 & 0 & 1.888 & 1 & .524 & 8 & 0 & 9 \\
\hline 429 & 1 & 9.693 & 1 & 5.862 & 1 & 1.273 & 1 & 0 & 1 \\
\hline 430 & 0 & 2.620 & 0 & 5.100 & 1 & 1.020 & 0 & 0 & 1 \\
\hline 430 & 1 & 4.970 & 1 & 4.760 & 1 & 1.100 & 1 & 1 & 1 \\
\hline 430 & 1 & 6.420 & 1 & 3.550 & 1 & 2.050 & 4 & 1 & 1 \\
\hline 430 & 1 & 4.280 & 1 & 4.790 & 1 & .750 & 5 & 0 & 1 \\
\hline 437 & 0 & 4.460 & 1 & 4.510 & 1 & 1.310 & 0 & 1 & 1 \\
\hline 437 & 1 & 8.240 & 1 & 3.250 & 1 & 2.050 & 4 & 1 & 1 \\
\hline 440 & 1 & 5.722 & 1 & 3.338 & 1 & .965 & 8 & 0 & 1 \\
\hline 441 & 0 & 3.360 & 0 & 5.190 & 1 & 2.050 & 0 & 0 & 1 \\
\hline 441 & 0 & 6.630 & 1 & 3.760 & 1 & 1.020 & 0 & 0 & 1 \\
\hline 441 & 1 & 6.660 & 1 & 5.130 & 1 & 1.080 & 5 & 0 & 1 \\
\hline 443 & 0 & 4.360 & 1 & 3.820 & 1 & 1.870 & 0 & 1 & 1 \\
\hline 447 & 0 & 2.810 & 1 & 4.980 & 1 & 1.280 & 0 & 1 & 1 \\
\hline 447 & 0 & 5.340 & 0 & 5.170 & 1 & 1.280 & 0 & 0 & 1 \\
\hline 447 & 0 & 5.763 & 1 & 2.501 & 1 & .646 & 4 & 0 & 1 \\
\hline 449 & 0 & 3.020 & 1 & 5.950 & 1 & 1.260 & 0 & 0 & 1 \\
\hline 449 & 0 & 4.680 & 1 & 4.730 & 1 & 1.990 & 0 & 0 & 1 \\
\hline 449 & 0 & 5.080 & 1 & 4.480 & 1 & 2.020 & 0 & 1 & 1 \\
\hline 449 & 0 & 5.390 & 1 & 5.450 & 1 & .510 & 0 & 0 & 1 \\
\hline 449 & 1 & 4.690 & 1 & 4.330 & 1 & 1.360 & 1 & 1 & 1 \\
\hline 449 & 1 & 4.310 & 1 & 4.290 & 1 & .990 & 3 & 0 & 1 \\
\hline 449 & 1 & 8.080 & 1 & 4.780 & 1 & 1.350 & 4 & 0 & 1 \\
\hline 456 & 0 & 3.570 & 0 & 7.200 & 1 & 1.710 & 0 & 1 & 1 \\
\hline 456 & 0 & 5.690 & 1 & 5.650 & 1 & 1.830 & 0 & 1 & 1 \\
\hline 461 & 0 & 4.500 & 1 & 4.550 & 1 & 2.090 & 0 & 1 & 1 \\
\hline 461 & 0 & 4.500 & 1 & 4.670 & 1 & 1.520 & 0 & 0 & 1 \\
\hline 461 & 0 & 5.500 & 1 & 4.610 & 1 & 1.330 & 0 & 0 & 1 \\
\hline 461 & 0 & 6.500 & 1 & 5.970 & 1 & 2.440 & 0 & 1 & 1 \\
\hline 461 & 0 & 6.500 & 1 & 6.300 & 1 & 1.160 & 0 & 2 & 1 \\
\hline 461 & 0 & 7.500 & 1 & 6.680 & 1 & 2.490 & 0 & 1 & 1 \\
\hline 464 & 1 & 9.720 & 1 & 4.750 & 1 & 2.270 & 4 & 1 & 1 \\
\hline 465 & 0 & 5.490 & 1 & 3.200 & 1 & 1.580 & 0 & 1 & 1 \\
\hline 465 & 1 & 5.450 & 1 & 3.210 & 1 & 1.370 & 4 & 0 & 1 \\
\hline 467 & 0 & 2.620 & 1 & 4.600 & 1 & 1.800 & 0 & 1 & 1 \\
\hline 467 & 0 & 3.410 & 0 & 2.760 & 1 & .750 & 0 & 0 & 1 \\
\hline 467 & 0 & 3.950 & 1 & 2.470 & 1 & .770 & 0 & 0 & 1 \\
\hline 471 & 1 & 5.460 & 1 & 2.900 & 1 & 1.250 & 4 & 0 & 1 \\
\hline 474 & 0 & 5.360 & 1 & 3.270 & 1 & 1.160 & 0 & 0 & 1 \\
\hline 474 & 0 & 5.830 & 1 & 4.320 & 1 & 1.050 & 0 & 0 & 1 \\
\hline 474 & 1 & 5.390 & 1 & 3.690 & 1 & .940 & 4 & 0 & 1 \\
\hline 481 & 1 & 4.003 & 0 & 2.597 & 1 & .709 & 8 & 0 & 1 \\
\hline 482 & 1 & 7.350 & 1 & 5.860 & 1 & 2.180 & 4 & 2 & 1 \\
\hline 485 & 0 & 4.130 & 0 & 2.430 & 1 & .810 & 0 & 0 & 1 \\
\hline 485 & 0 & 4.770 & 0 & 2.950 & 1 & .570 & 0 & 0 & 1 \\
\hline
\end{tabular}


Table E-1. continued...

\begin{tabular}{|c|c|c|c|c|c|c|c|c|c|}
\hline $\begin{array}{c}\text { 411TG } \\
\#\end{array}$ & \begin{tabular}{|c|}
$\begin{array}{c}\text { Complete } \\
\text { Length }\end{array}$ \\
\end{tabular} & $\begin{array}{l}\text { Length } \\
\mathrm{cm} .\end{array}$ & $\begin{array}{c}\begin{array}{c}\text { Complete } \\
\text { Width }\end{array} \\
\end{array}$ & $\begin{array}{c}\text { Width } \\
\mathrm{cm} .\end{array}$ & $\begin{array}{l}\text { Complete } \\
\text { Thickness }\end{array}$ & $\begin{array}{c}\text { Thickness } \\
\mathrm{cm} .\end{array}$ & $\begin{array}{l}\text { Biface } \\
\text { Shape }\end{array}$ & $\begin{array}{c}\text { Cortex } \\
\text { Present } \\
\end{array}$ & $\begin{array}{c}\text { Material } \\
\text { Types } \\
\end{array}$ \\
\hline 485 & 1 & 5.190 & 1 & 4.860 & 1 & 1.520 & 1 & 1 & 9 \\
\hline 485 & 1 & 3.737 & 1 & 3.408 & 1 & .591 & 4 & 0 & 1 \\
\hline 485 & 0 & 2.430 & 0 & 1.190 & 1 & .450 & 8 & 0 & 1 \\
\hline 486 & 0 & 1.953 & 1 & 2.979 & 1 & .689 & 0 & 0 & 1 \\
\hline 488 & 1 & 4.279 & 1 & 1.728 & 1 & .759 & 8 & 0 & 1 \\
\hline 489 & 0 & 2.830 & 0 & 3.400 & 1 & .660 & 0 & 0 & 1 \\
\hline 489 & 0 & 3.930 & 0 & 3.800 & 1 & .910 & 0 & 1 & 9 \\
\hline 489 & 1 & 4.730 & 1 & 3.810 & 1 & .580 & 4 & 0 & 1 \\
\hline 489 & 1 & 5.400 & 1 & 3.590 & 1 & 1.200 & 4 & 0 & 1 \\
\hline 489 & 1 & 5.850 & 1 & 4.510 & 1 & 1.820 & 5 & 0 & 1 \\
\hline 489 & 1 & 3.770 & 1 & 2.407 & 1 & .803 & 8 & 0 & 1 \\
\hline 489 & 0 & 4.207 & 1 & 2.491 & 1 & .709 & 8 & 0 & 1 \\
\hline 491 & 1 & 6.400 & 1 & 3.200 & 1 & 1.330 & 0 & 1 & 1 \\
\hline 495 & 1 & 11.950 & 1 & 5.010 & 1 & 2.970 & 4 & 0 & 1 \\
\hline 496 & 0 & 2.650 & 1 & .870 & 1 & .480 & 0 & 0 & 1 \\
\hline 496 & 0 & 5.600 & 1 & 4.740 & 1 & 1.400 & 0 & 1 & 1 \\
\hline 496 & 1 & 6.180 & 1 & 5.430 & 1 & 1.670 & 1 & 1 & 1 \\
\hline 497 & 1 & 5.876 & 1 & 3.343 & 1 & .808 & 8 & 0 & 1 \\
\hline 500 & 0 & 2.160 & 1 & 2.010 & 1 & .340 & 0 & 0 & 9 \\
\hline 500 & 0 & 2.990 & 1 & 2.280 & 1 & .850 & 0 & 0 & 1 \\
\hline 500 & 1 & 6.600 & 1 & 6.160 & 1 & 1.350 & 1 & 0 & 1 \\
\hline 504 & 1 & 5.060 & 1 & 3.493 & 1 & .572 & 3 & 0 & 2 \\
\hline 504 & 0 & 1.120 & 0 & .500 & 0 & .790 & 8 & 0 & 1 \\
\hline 504 & 0 & 2.810 & 1 & 2.561 & 1 & .816 & 8 & 0 & 9 \\
\hline 506 & 0 & 1.630 & 1 & 1.570 & 1 & .420 & 0 & 0 & 1 \\
\hline 506 & 0 & 1.990 & 0 & 2.110 & 1 & .250 & 0 & 0 & 9 \\
\hline 506 & 0 & 2.740 & 1 & 4.640 & 1 & .950 & 0 & 1 & 1 \\
\hline 506 & 0 & 2.940 & 1 & 3.110 & 1 & .710 & 0 & 0 & 9 \\
\hline 506 & 0 & 3.070 & 1 & 1.910 & 1 & .620 & 0 & 0 & 3 \\
\hline 506 & 0 & 3.370 & 0 & 5.280 & 1 & .960 & 0 & 0 & 1 \\
\hline 506 & 0 & 4.490 & 1 & 3.350 & 1 & 1.100 & 0 & 1 & 1 \\
\hline 506 & 1 & 7.180 & 1 & 3.980 & 1 & 1.270 & 1 & 2 & 1 \\
\hline 506 & 1 & 4.940 & 1 & 5.040 & 1 & 1.220 & 5 & 1 & 1 \\
\hline 506 & 0 & 2.688 & 1 & 1.667 & 1 & .717 & 8 & 0 & 9 \\
\hline 506 & 0 & 2.962 & 1 & 2.124 & 1 & .347 & 8 & 0 & 2 \\
\hline 507 & 1 & 7.110 & 1 & 5.490 & 1 & 1.220 & 1 & 1 & 1 \\
\hline 508 & 1 & 6.380 & 1 & 4.080 & 1 & 1.080 & 4 & 0 & 1 \\
\hline 511 & 0 & 3.190 & 1 & 2.940 & 1 & .600 & 0 & 0 & 1 \\
\hline 511 & 0 & 4.650 & 1 & 2.440 & 1 & 1.690 & 0 & 0 & 1 \\
\hline 511 & 1 & 5.710 & 1 & 4.830 & 1 & 1.660 & 1 & 1 & 9 \\
\hline 512 & 0 & 3.590 & 1 & 4.690 & 1 & 2.770 & 0 & 1 & 1 \\
\hline 512 & 1 & 5.250 & 1 & 4.290 & 1 & 1.160 & 5 & 1 & 9 \\
\hline 512 & 0 & 5.717 & 1 & 3.493 & 1 & .877 & 8 & 0 & 1 \\
\hline 513 & 0 & 3.510 & 1 & 3.140 & 1 & .480 & 0 & 0 & 1 \\
\hline 513 & 0 & 3.800 & 1 & 2.820 & 1 & 1.430 & 0 & 0 & 1 \\
\hline 513 & 1 & 5.500 & 1 & 2.540 & 1 & .960 & 5 & 0 & 1 \\
\hline 513 & 1 & 5.500 & 1 & 4.340 & 1 & 1.220 & 5 & 0 & 1 \\
\hline 515 & 0 & 5.540 & 1 & 4.570 & 1 & 1.860 & 0 & 1 & 1 \\
\hline 515 & 1 & 9.340 & 1 & 5.690 & 1 & 1.880 & 4 & 1 & 1 \\
\hline 515 & 1 & 5.970 & 1 & 6.200 & 1 & 1.170 & 5 & 1 & 1 \\
\hline 517 & 0 & 2.656 & 0 & 3.332 & 1 & .696 & 8 & 0 & 1 \\
\hline
\end{tabular}


Table E-1. continued...

\begin{tabular}{|c|c|c|c|c|c|c|c|c|c|}
\hline $\begin{array}{c}\text { 41TG } \\
\# \\
\end{array}$ & $\begin{array}{c}\text { Complete } \\
\text { Length }\end{array}$ & $\begin{array}{c}\text { Length } \\
\mathrm{cm} .\end{array}$ & \begin{tabular}{|c|}
$\begin{array}{c}\text { Complete } \\
\text { Width }\end{array}$ \\
\end{tabular} & $\begin{array}{l}\text { Width } \\
\mathrm{cm} .\end{array}$ & $\begin{array}{l}\text { Complete } \\
\text { Thickness }\end{array}$ & $\begin{array}{c}\text { Thickness } \\
\mathrm{cm} .\end{array}$ & $\begin{array}{l}\text { Biface } \\
\text { Shape } \\
\end{array}$ & $\begin{array}{c}\text { Cortex } \\
\text { Present } \\
\end{array}$ & $\begin{array}{c}\text { Material } \\
\text { Types }\end{array}$ \\
\hline 517 & 0 & 2.950 & 1 & 4.401 & 1 & .914 & 8 & 0 & 9 \\
\hline 518 & 0 & 2.145 & 1 & 2.134 & 1 & .548 & 0 & 0 & 1 \\
\hline 518 & 0 & 2.880 & 1 & 2.890 & 1 & .390 & 0 & 0 & 1 \\
\hline 518 & 0 & 3.600 & 1 & 3.950 & 1 & 1.110 & 0 & 0 & 2 \\
\hline 518 & 1 & 5.440 & 1 & 4.220 & 1 & 1.250 & 5 & 1 & 2 \\
\hline 519 & 0 & 3.080 & 1 & 6.590 & 1 & 1.480 & 0 & 0 & 2 \\
\hline 523 & 1 & 8.250 & 1 & 7.250 & 1 & 2.320 & 4 & 0 & 1 \\
\hline 523 & 0 & 1.920 & 0 & 1.584 & 1 & .612 & 8 & 0 & 1 \\
\hline 523 & 0 & 2.879 & 1 & 2.633 & 1 & .816 & 8 & 0 & 1 \\
\hline 524 & 0 & 2.110 & 1 & 1.940 & 1 & .720 & 0 & 0 & 1 \\
\hline 524 & 1 & 5.675 & 1 & 2.950 & 1 & .745 & 8 & 0 & 1 \\
\hline 525 & 0 & 3.110 & 1 & 5.560 & 1 & .820 & 0 & 0 & 1 \\
\hline 525 & 0 & 3.303 & 0 & 2.584 & 1 & .690 & 8 & 0 & 1 \\
\hline 526 & 0 & 2.380 & 1 & 2.350 & 1 & .800 & 0 & 0 & 9 \\
\hline 526 & 0 & 4.580 & 1 & 7.460 & 1 & 1.700 & 0 & 0 & 9 \\
\hline 526 & 1 & 4.090 & 1 & 3.090 & 1 & .450 & 3 & 0 & 1 \\
\hline 526 & 0 & 2.242 & 1 & 3.891 & 1 & .784 & 8 & 0 & 9 \\
\hline 528 & 0 & 3.670 & 1 & 5.100 & 1 & 1.340 & 0 & 0 & 1 \\
\hline 530 & 0 & 2.450 & 1 & 1.800 & 1 & .350 & 8 & 0 & 1 \\
\hline 530 & 0 & 2.665 & 1 & 2.010 & 1 & .615 & 8 & 0 & 1 \\
\hline 531 & 1 & 3.190 & 1 & 2.570 & 1 & .720 & 0 & 0 & 1 \\
\hline 531 & 0 & 3.480 & 1 & 4.400 & 1 & .780 & 0 & 1 & 1 \\
\hline 531 & 0 & 3.500 & 1 & 6.060 & 1 & 1.660 & 0 & 0 & 9 \\
\hline 531 & 0 & 3.730 & 0 & 1.550 & 1 & .740 & 0 & 0 & 1 \\
\hline 531 & 0 & 3.980 & 1 & 4.030 & 1 & 1.560 & 0 & 1 & 2 \\
\hline 531 & 0 & 4.190 & 0 & 2.460 & 1 & .450 & 0 & 0 & 1 \\
\hline 531 & 0 & 4.370 & 1 & 4.260 & 1 & 1.560 & 0 & 1 & 1 \\
\hline 531 & 1 & 5.960 & 1 & 3.700 & 1 & 1.600 & 1 & 0 & 1 \\
\hline 531 & 0 & 2.062 & 0 & 3.663 & 1 & .735 & 8 & 0 & 1 \\
\hline 533 & 0 & 2.370 & 1 & 2.330 & 1 & .590 & 0 & 0 & 1 \\
\hline 533 & 0 & 6.510 & 1 & 3.910 & 1 & 1.050 & 0 & 0 & 1 \\
\hline 533 & 1 & 5.610 & 1 & 3.240 & 1 & .490 & 4 & 0 & 9 \\
\hline 533 & 1 & 8.640 & 1 & 3.890 & 1 & 1.970 & 4 & 1 & 1 \\
\hline 533 & 1 & 6.550 & 1 & 2.750 & 1 & .820 & 5 & 0 & 9 \\
\hline 534 & 0 & 4.060 & 0 & 3.642 & 1 & .721 & 0 & 0 & 2 \\
\hline 534 & 0 & 3.948 & 0 & 2.865 & 1 & .568 & 3 & 0 & 1 \\
\hline 534 & 0 & 1.706 & 1 & 2.509 & 1 & .525 & 8 & 0 & 1 \\
\hline 534 & 0 & 3.346 & 0 & 2.970 & 1 & .777 & 8 & 0 & 1 \\
\hline 535 & 0 & 4.712 & 1 & 1.604 & 1 & .740 & 4 & 0 & 1 \\
\hline
\end{tabular}





\section{Appendix F: \\ Twin Buttes Archaeological Project}

\section{Unique Items}

Chipped stone Unique Items (UIs) discussed in the text are illustrated on the following pages. The diagnostics are arranged in chronological order, from Paleoindian through Late Prehistoric, followed by non-diagnostic tools. 


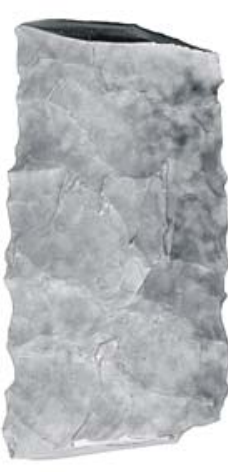

a

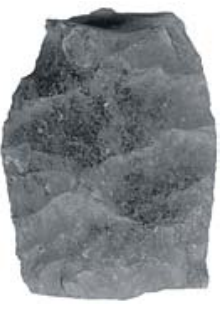

d
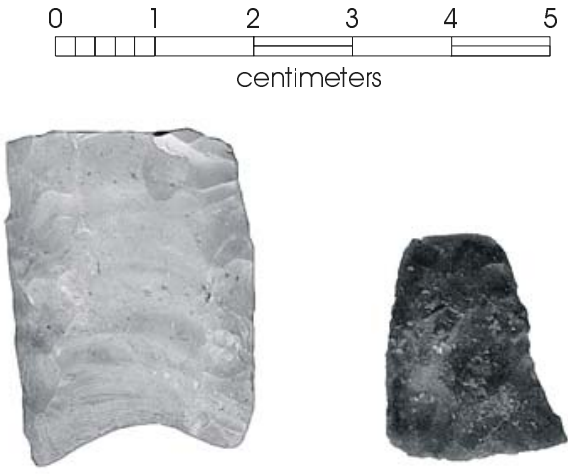

b

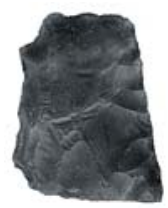

e

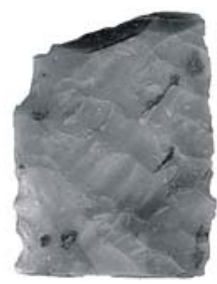

f

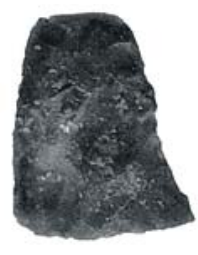

C

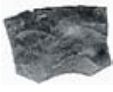

9

Figure F-1. Paleoindian points: (a) 41TG370, UI-7, Lanceolate; (b) 41TG378, UI-12, Clovis; (c) 41TG109, UI66, Angostura; (d) 41TG246, UI-35, Angostura-like; (e) 41TG523, UI-67, Late Paleo-like; (f) 41TG252, UI-60, Plainview-like; (g) 41TG534, UI-303, Barber or Golondrina-like. 


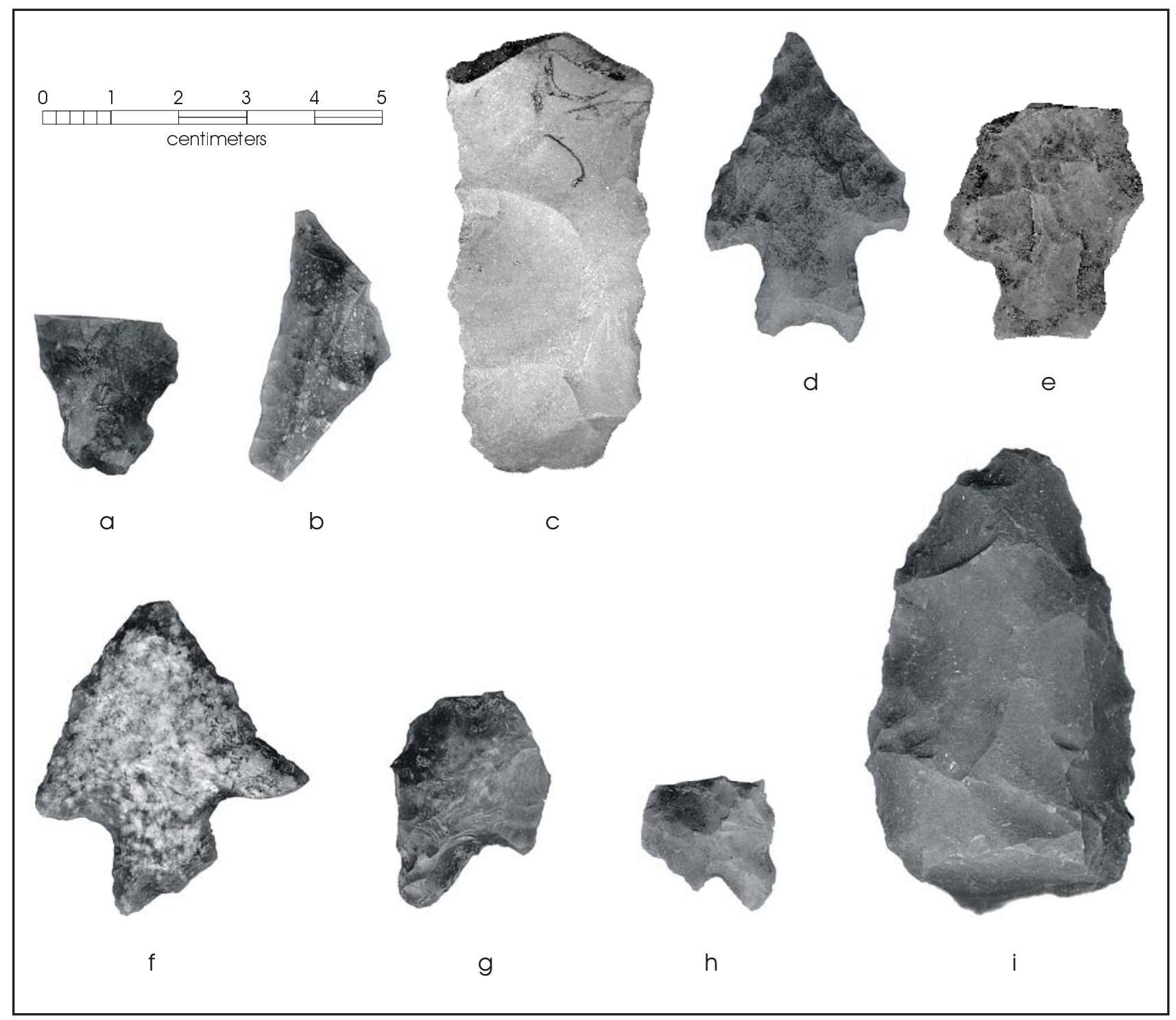

Figure F-2. Early Archaic points and tools: (a) 41TG362, UI-41, Uvalde-like; (b) 41TG373, UI-10, Early Triangular; (c) 41TG388, UI-20, Guadalupe Adze; (d) 41TG388, UI-70, Uvalde; (e) 41TG390, UI-21, Bandy-like; (f) 41TG424, UI-29, Martindale or Bandy; (g) 41TG449, UI-56, Baker-like; (h) 41TG247, UI-57, Martindale-like; (i) 41TG244, UI-319, Guadalupe Adze. 


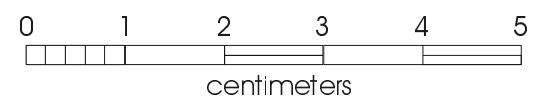

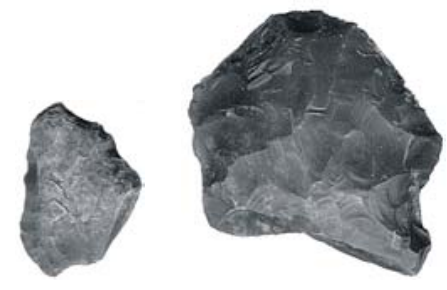

j

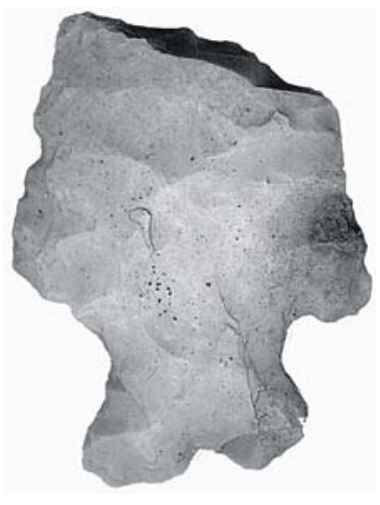

○

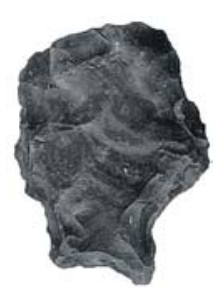

I

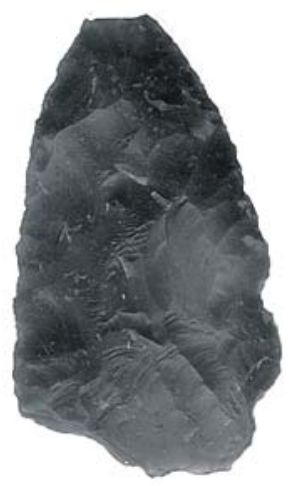

$\mathrm{p}$

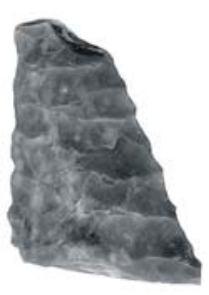

$\mathrm{m}$

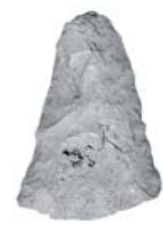

$\mathrm{n}$

Figure F-2 (continued). Early Archaic points and tools: (j) 41TG534, UI-308, Early Triangular-like; (k) 41TG523, UI-64, Gower-like; (l) 41TG530, UI-58, Val Verde; (m) 41TG369, UI-6, point medial fragment; (n) 41TG504, UI-305, Early Triangular [possibly Late Archaic Tortugas]; (o) 41TG529, UI-322, Martindale [possibly Late Archaic Frio]; (p) isolated find, UI-23, Early Triangular [possibly Late Archaic Tortugas]; (q) 41TG526, UI47, morphologically Early Archaic. 


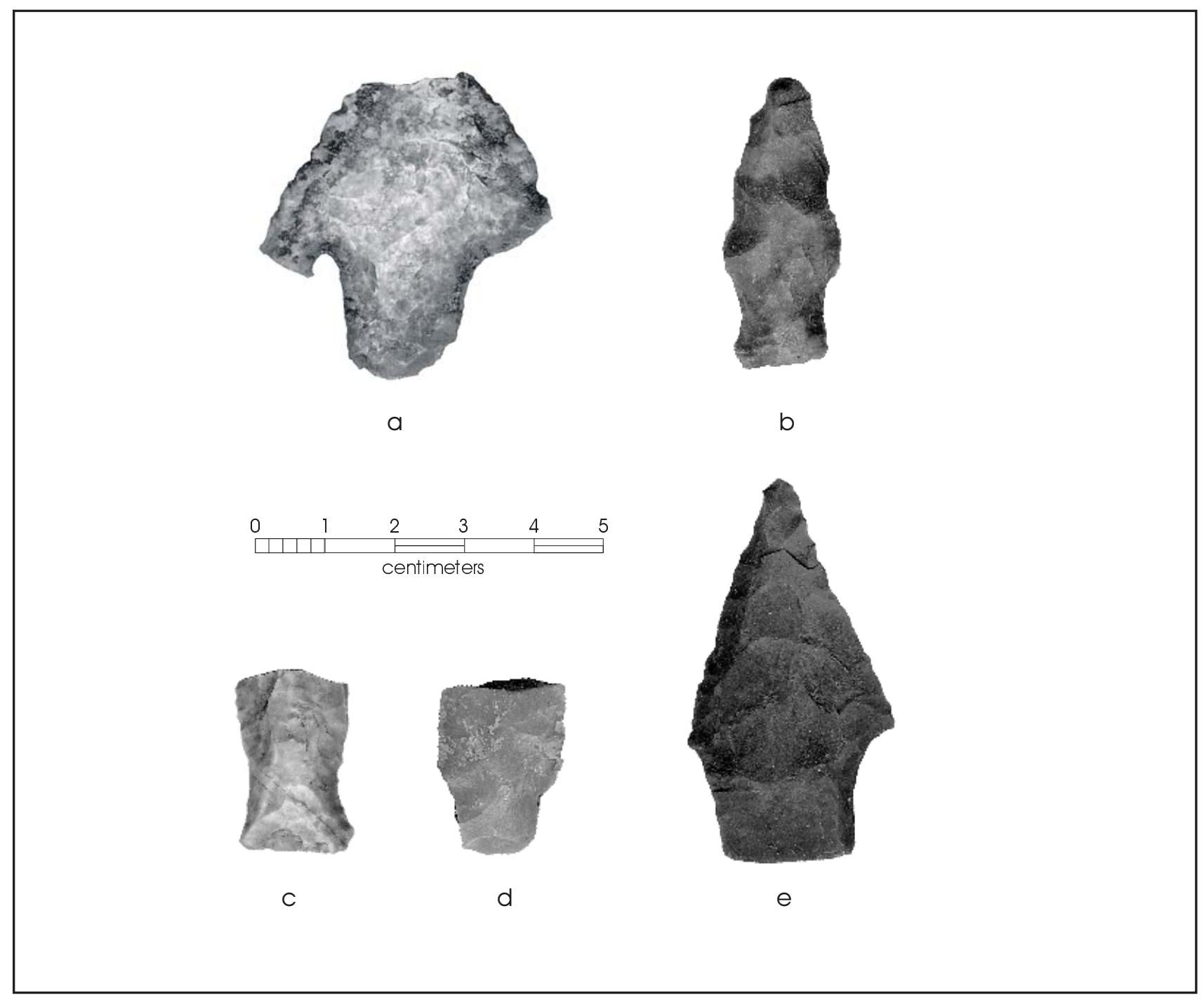

Figure F-3. Middle Archaic points: (a) 41TG364, UI-39, Andice; (b) 41TG488, UI-37, Pandale; (c) 41TG506, UI-302, Pandale; (d) 41TG530, UI-63, Pandale; (e) isolated find, UI-34, Nolan. 


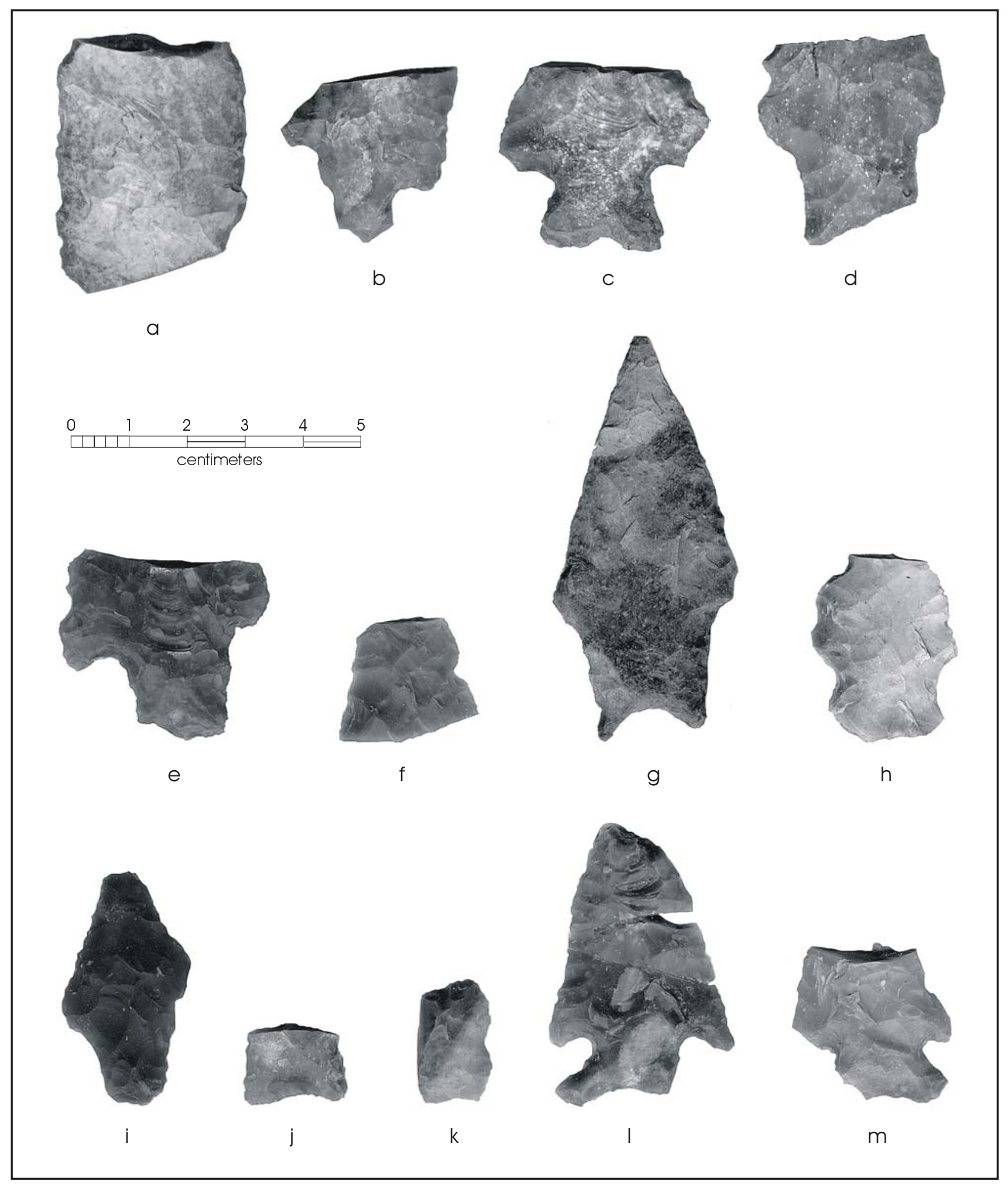

Figure F-4. Late Archaic points: (a) 41TG362, UI-1, Marcos/Marshall-like; (b) 41TG418, UI-26, Frio-like; (c) 41TG105, UI-40, Marshall; (d) 41TG365, UI-2, Marcos-Marshall-like; (e) 41TG367, UI-3, Marshall or Williams-like; (f) 41TG367, UI-5, morphologically Late Archaic; (g) 41TG382, UI-18, Pedernales; (h) 41TG391, UI-22, Trinity-like; (i) 41TG404, UI-49, Langtry; (j) 41TG405, UI-50, Marshall-like; (k) 41TG409, UI-65, Marcos-like; (l) 41TG410, UI-45, Frio-like; (m) 41TG412, UI-24, Ensor or Frio. 


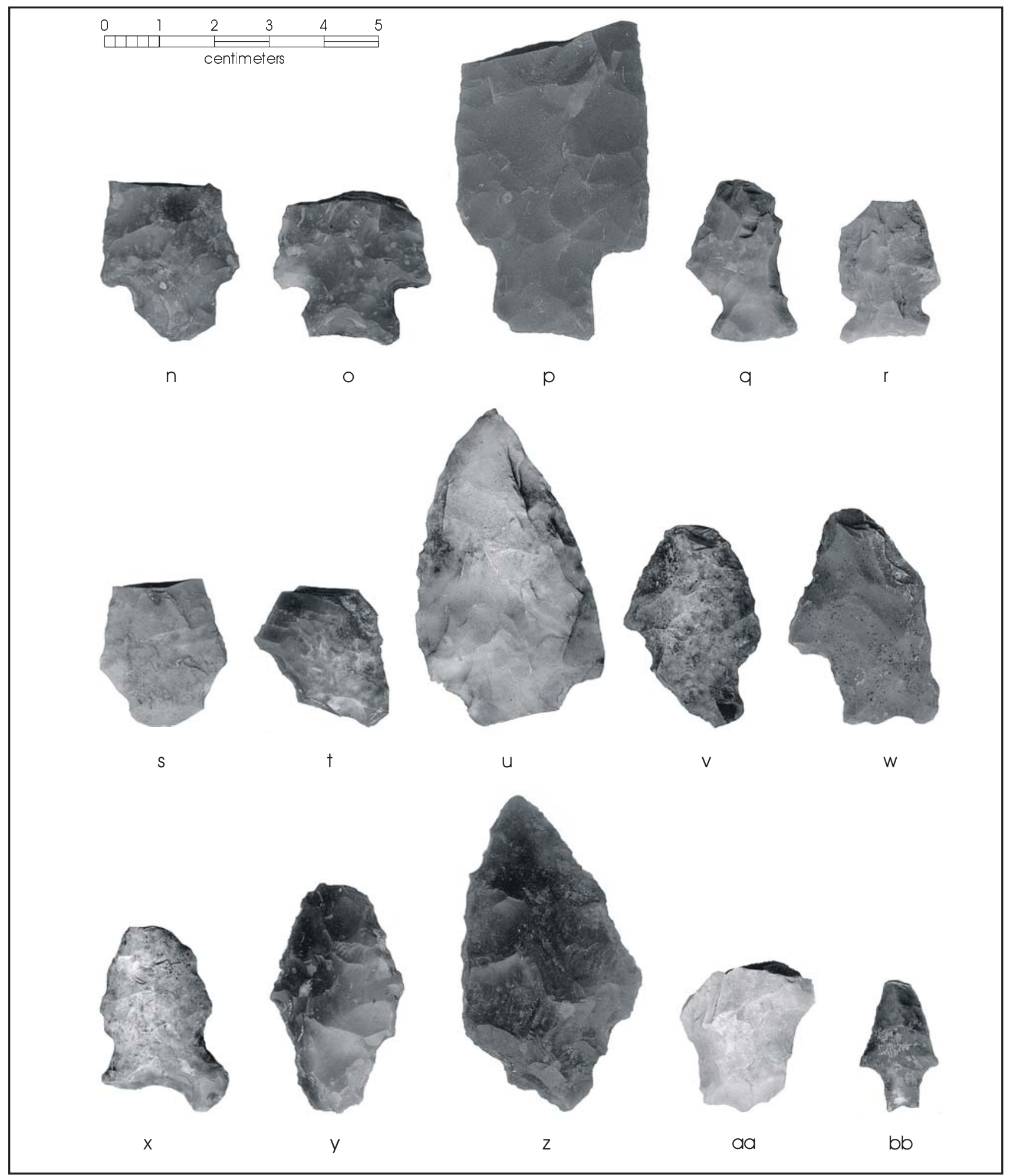

Figure F-5. Late Archaic points (continued): (n) 41TG412, UI-25, morphologically Late Archaic; (o) 41TG412, UI-68, Marshall; (p) 41TG424, UI-72, Marshall-like; (q) 41TG427, UI-28, Ensor-like; (r) 41TG427, UI-53, Fairland; (s) 41TG427, UI-54, morphologically Late Archaic; (t) 41TG110, UI-32, Frio-like; (u) 41TG440, UI31, Marshall or Williams-like; (v) 41TG247, UI-71, Frio-like; (w) 41TG481, UI-36, morphologically Late Archaic; (x) 41TG489, UI-38, Frio-like; (y) 41TG489, UI-52, Langtry; (z) 41TG497, UI-48, morphologically Late Archaic; (aa) 41TG504, UI-306, Bulverde-like; (bb) 41TG512, UI-310, Langtry. 


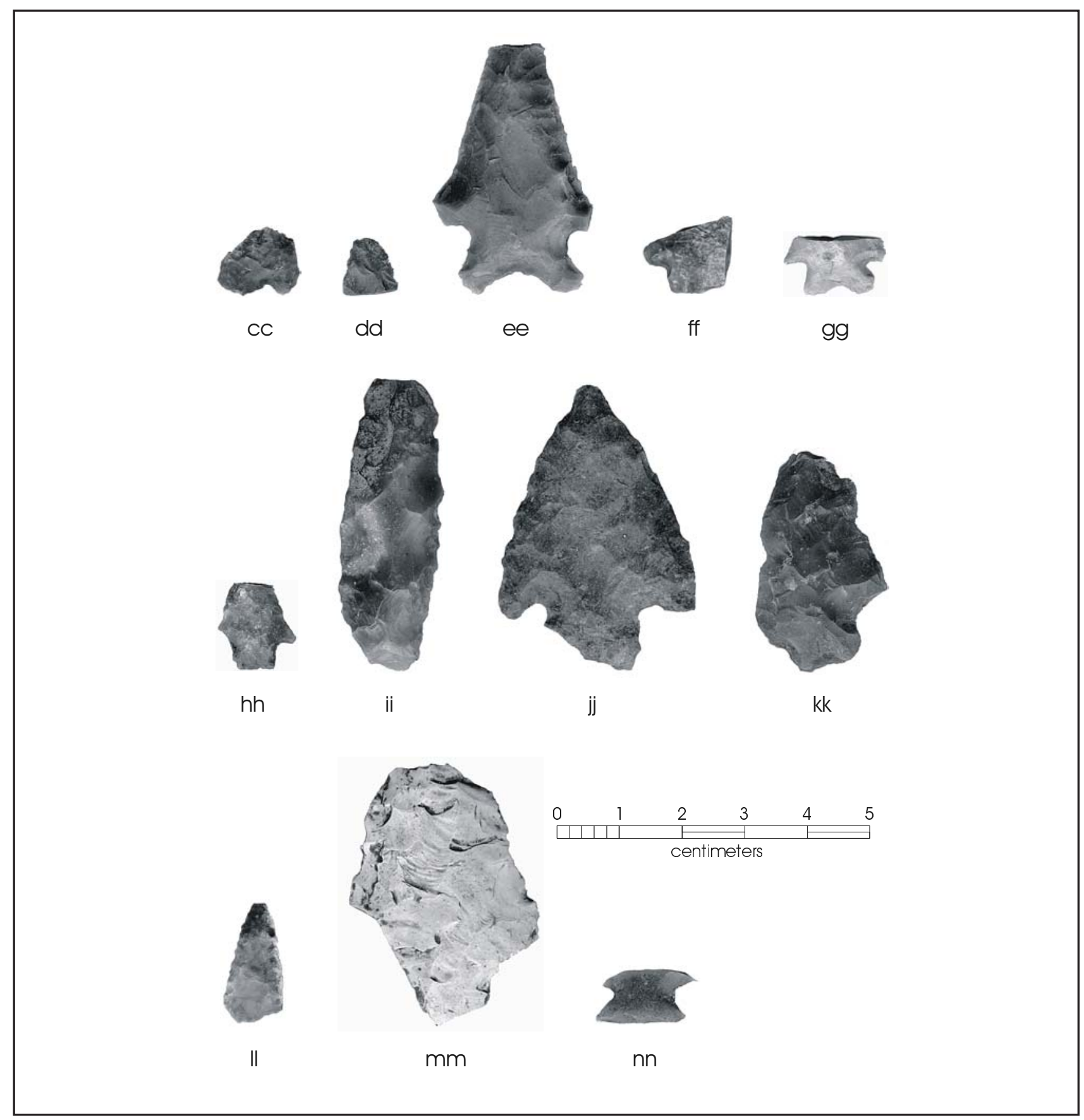

Figure F-6. Late Archaic points (continued): (cc) 41TG517, UI-312, Marcos or Williams-like; (dd) 41TG518, UI-46, morphologically Late Archaic; (ee) 41TG252, UI-323, Frio; (ff) 41TG525, UI-316, morphologically Late Archaic, burinated; (gg) 41TG526, UI-317, Frio-like; (hh) 41TG534, UI-304, Marcos or Marshall-like; (ii) 41TG535, UI-61, Pedernales-like; (jj) isolated find, UI-19, Marshall or Williams-like; (kk) isolated find, UI33, morphologically Late Archaic; (Il) isolated find, UI-314, Tortugas-like; (mm) isolated find, UI-324, morphologically Late Archaic; (nn) 41TG389, UI-69, Ensor or Ellis [possibly Late Prehistoric Scallorn]. 


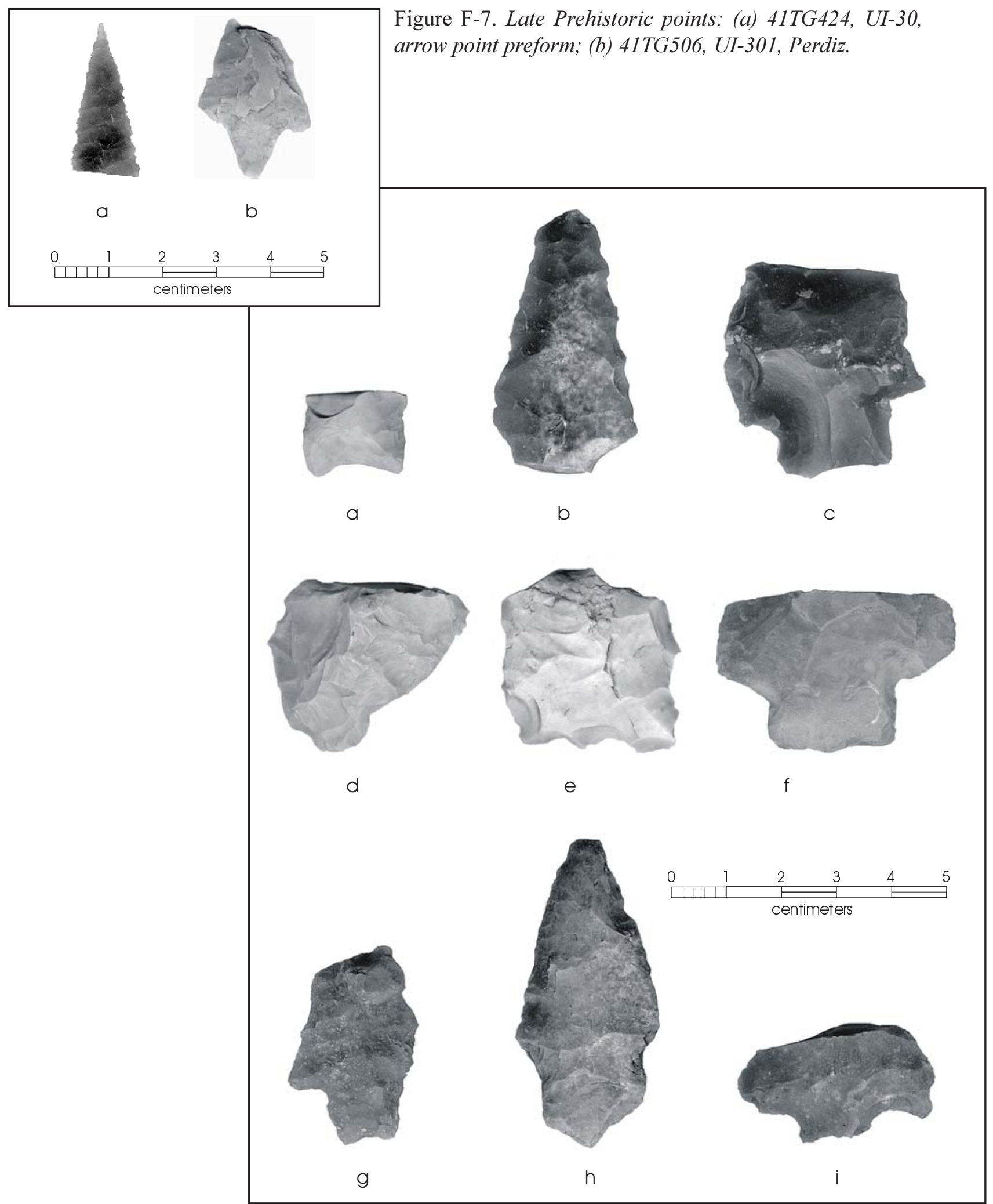

Figure F-8. Untypeable points: (a) 41TG367, UI-4, point base; (b) 41TG253, UI-75, point fragment; (c) 41TG377, UI-11, dart point preform base; (d) isolated find, UI-311, point fragment; (e) 41TG534, UI-307, point base; (f) 41TG517, UI-313, dart point base; (g) 41TG389, UI-44, dart point fragment; (h) 41TG524, UI-73, dart point fragment (possible Pedernales); (i) 41TG531, UI-62, dart point fragment. 


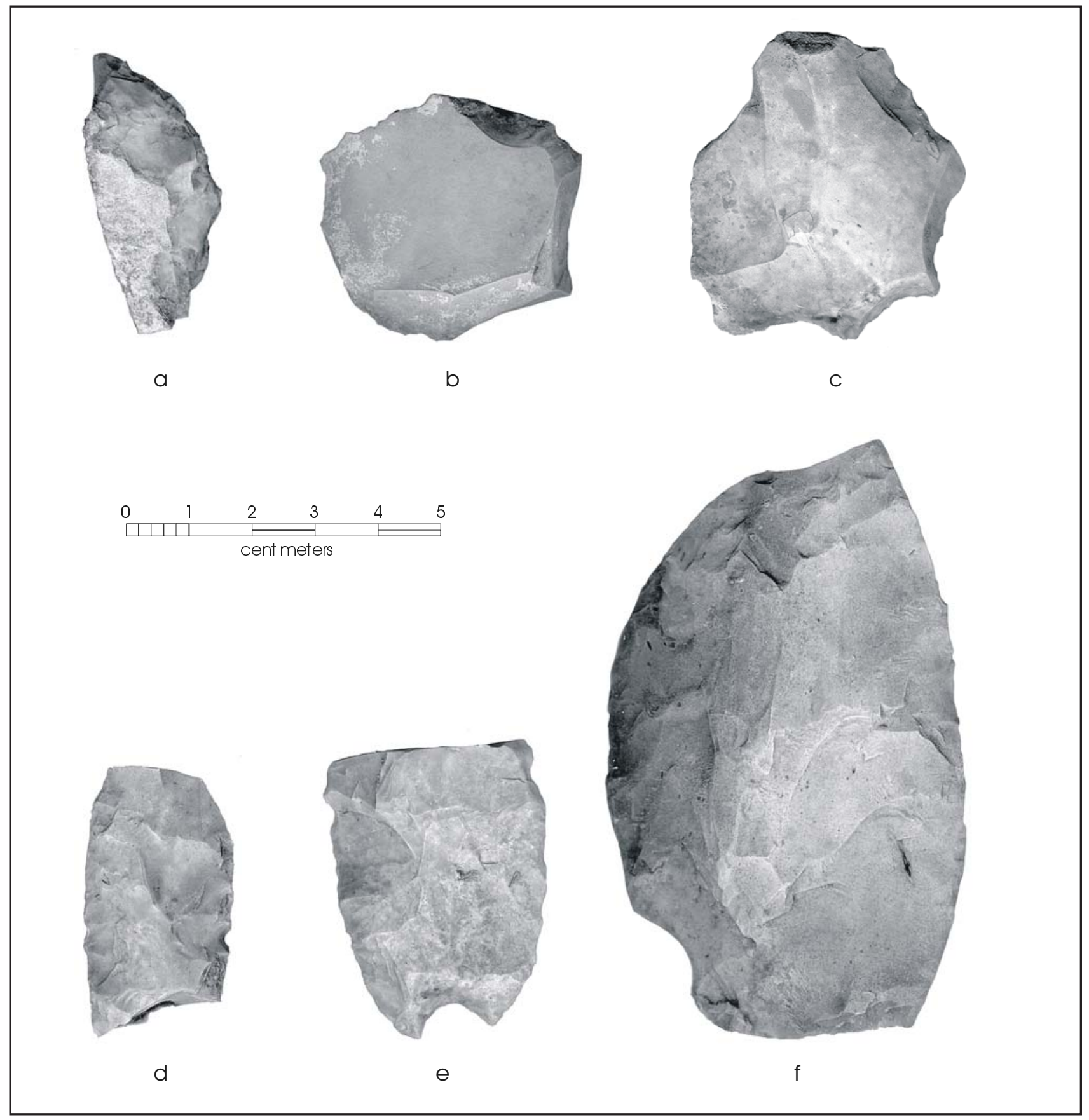

Figure F-9. Bifaces: (a-e) 41TG378, UIs 13, 14, 17, 43, and 59, respectively; (f) 41TG429, UI-55. 


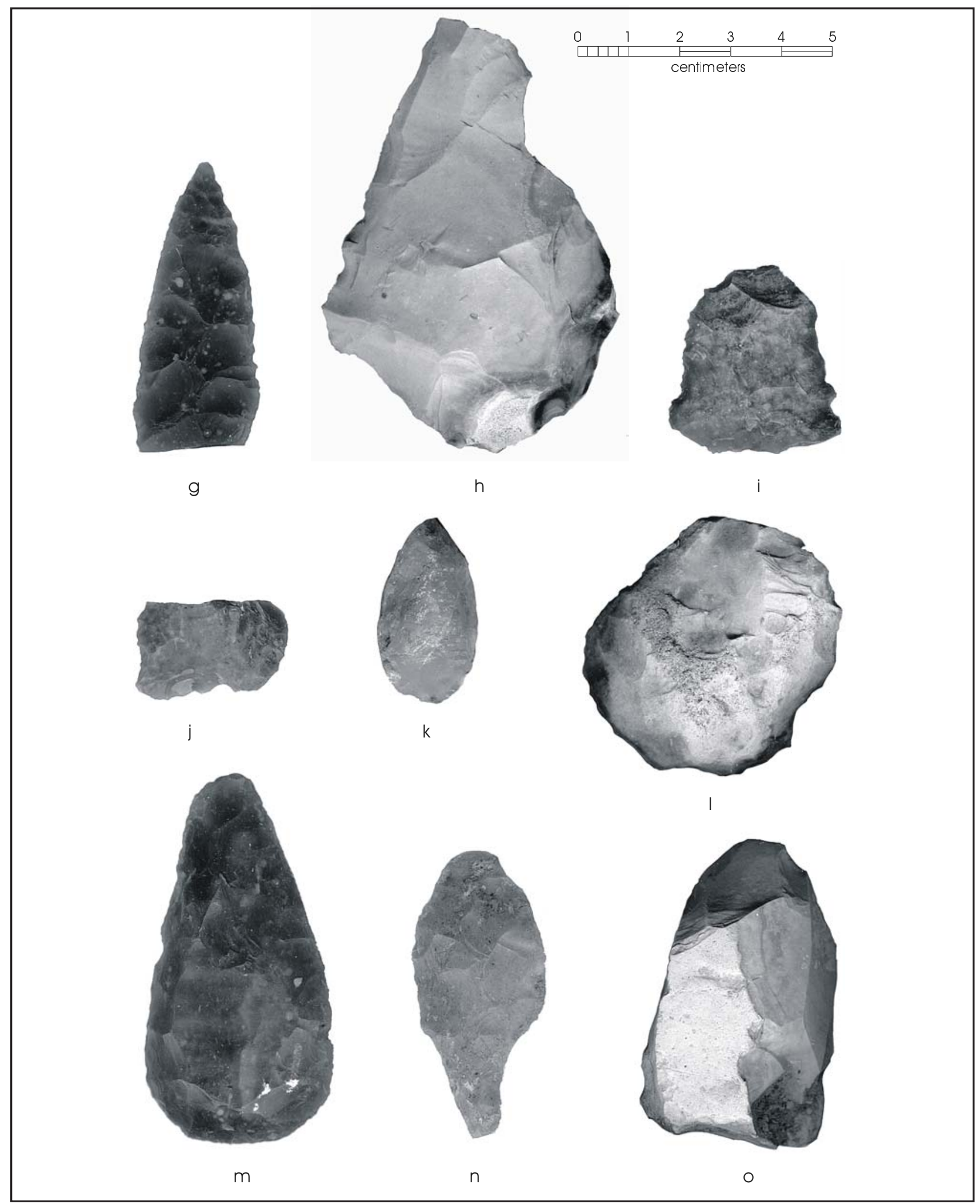

Figure F-10. Bifaces (continued): (g) 41TG447, UI-74; (h) isolated find, UI-309; (i) 41TG485, UI-320; (j) 41TG486, UI-321; (k) 41TG378, UI-15, made from quartz; (l) 41TG420, UI-27, scraper; (m) 41TG252, UI-325, scraper; (n) 41TG382, UI-326, burin; (o) 41TG378, UI-16, core tool/gouge. 


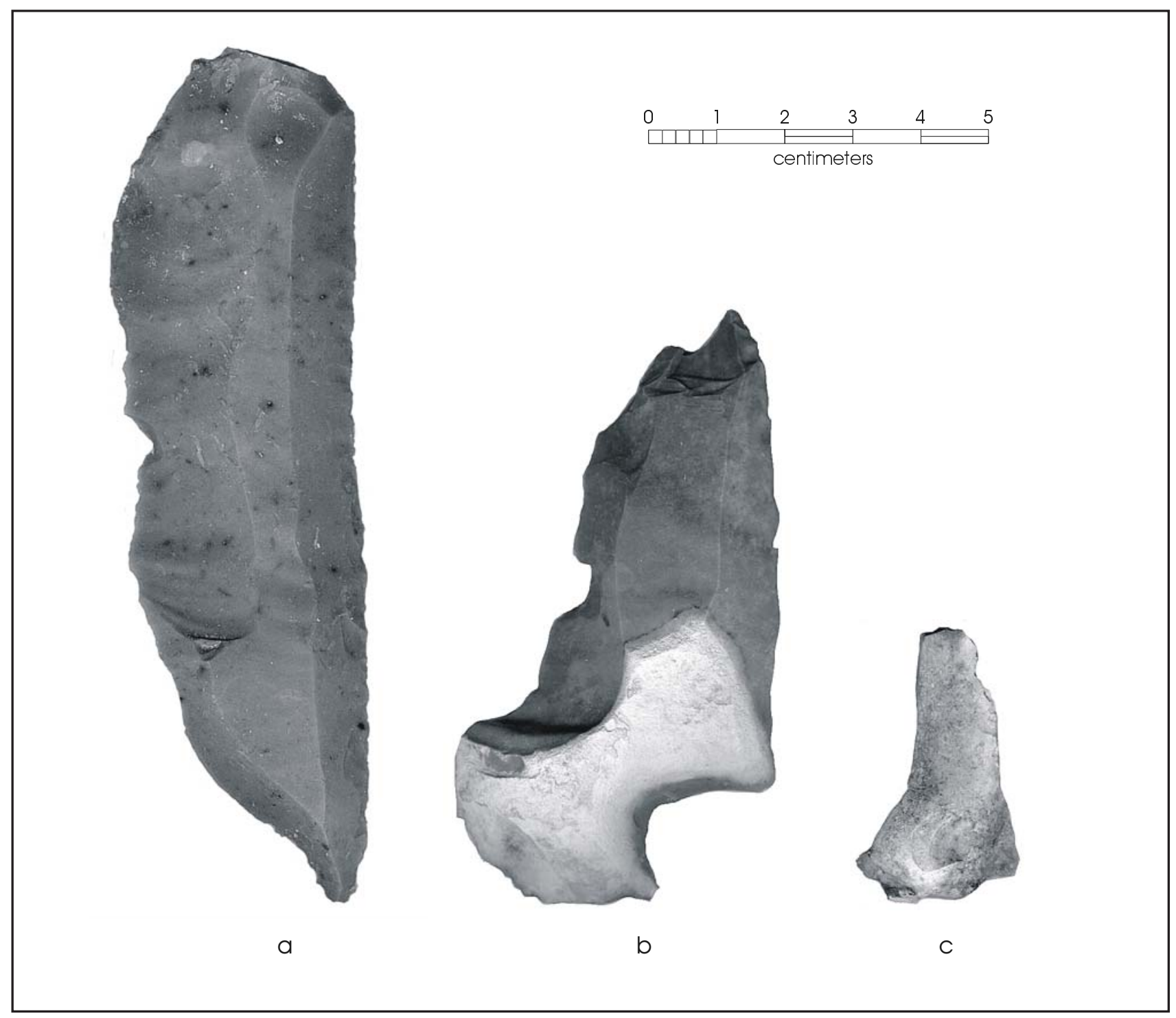

Figure F-11. Unifaces: (a) 41TG513, UI-76, knife; (b) 41TG372, UI-9, flake tool; (c) 41TG378, UI-42, flake tool. 


\section{Appendix G: \\ Twin Buttes Archaeological Project}

Isolated Finds

This appendix provides individual summaries for all 393 isolated finds. The appendix consists of a table (Table G-1) with isolate information and a map (Figure G-1) showing the distribution of the isolated finds.

Table G-1 provides the UTM northing and easting for each isolate, maximum artifact length in $\mathrm{cm}$, cortex percentage, and artifact type. The codes in the artifact type field are as follows: $1=$ flake, $2=$ core or tested cobble, 4 = biface, $5=$ uniface, $6=$ retouched/utilized item, $10=$ projectile point. Note that projectile points were collected, so no attributes were recorded for the eight specimens in this table. Note that in a small number of cases, data are missing for a given attribute.

Figure G-1 is a map which provides distributional information. 
Table G-1. Data for Isolated Finds

\begin{tabular}{|c|c|c|c|c|}
\hline UTM Northing & UTM Easting & Length $(\mathrm{cm})$ & Cortex (\%) & Artifact Tvpe \\
\hline 3460162 & 356860 & & & 10 \\
\hline 3461440 & 356893 & 8.50 & 20.00 & 2 \\
\hline 3461922 & 357078 & 11.50 & 60.00 & 2 \\
\hline 3462467 & 357751 & 1.50 & .00 & 6 \\
\hline 3462563 & 357122 & 7.50 & 20.00 & 1 \\
\hline 3462765 & 357613 & 7.50 & 60.00 & 2 \\
\hline 3462772 & 357632 & 4.50 & 20.00 & 6 \\
\hline 3463392 & 358167 & & & 10 \\
\hline 3463821 & 357539 & 3.50 & 20.00 & 1 \\
\hline 3463979 & 357561 & 6.50 & 20.00 & 1 \\
\hline 3464001 & 357560 & 3.50 & 20.00 & 1 \\
\hline 3464019 & 359482 & 2.50 & 10.00 & 6 \\
\hline 3464045 & 357546 & 4.50 & .00 & 4 \\
\hline 3464112 & 359808 & 2.50 & 10.00 & 1 \\
\hline 3464141 & 357556 & 7.50 & .00 & 1 \\
\hline 3464247 & 356070 & 5.50 & 70.00 & 2 \\
\hline 3464256 & 359831 & 5.50 & 20.00 & 6 \\
\hline 3464297 & 358219 & 4.50 & 40.00 & 1 \\
\hline 3464338 & 358226 & 4.50 & 50.00 & 5 \\
\hline 3465073 & 359057 & 4.50 & 10.00 & 6 \\
\hline 3465074 & 359061 & 3.50 & .00 & 1 \\
\hline 3465079 & 359044 & 2.50 & 10.00 & 6 \\
\hline 3465189 & 359137 & 3.50 & 30.00 & 1 \\
\hline 3465208 & 359132 & 1.50 & & 1 \\
\hline 3465354 & 359229 & 6.50 & 10.00 & 4 \\
\hline 3465404 & 359222 & 9.50 & 20.00 & 2 \\
\hline 3465497 & 358364 & 7.50 & 10.00 & 5 \\
\hline 3465500 & 358374 & 4.50 & 10.00 & 5 \\
\hline 3465513 & 358345 & 4.50 & .00 & 1 \\
\hline 3465523 & 358975 & 7.50 & 50.00 & 1 \\
\hline 3465524 & 358977 & 2.50 & 20.00 & 1 \\
\hline 3465553 & 358177 & 5.50 & 10.00 & 1 \\
\hline 3465559 & 359399 & 7.50 & 20.00 & 2 \\
\hline 3465560 & 359400 & 5.50 & 40.00 & 5 \\
\hline 3465566 & 359415 & 4.50 & 30.00 & 4 \\
\hline 3465588 & 358982 & 5.50 & .00 & 5 \\
\hline 3465592 & 359097 & 2.50 & .00 & 6 \\
\hline 3465599 & 358217 & 9.50 & 10.00 & 1 \\
\hline 3465601 & 358945 & 6.50 & 10.00 & 5 \\
\hline 3465622 & 358235 & 8.50 & 30.00 & 1 \\
\hline 3465650 & 358202 & 4.50 & 60.00 & 1 \\
\hline 3465682 & 358172 & 10.50 & 10.00 & 4 \\
\hline 3465878 & 358523 & 3.50 & 60.00 & 1 \\
\hline 3466013 & 359010 & 2.50 & 10.00 & 1 \\
\hline 3466014 & 359007 & 7.50 & 40.00 & 1 \\
\hline 3466015 & 359008 & 3.50 & .00 & 5 \\
\hline 3466021 & 358959 & 5.50 & .00 & 6 \\
\hline 3466022 & 358967 & 3.50 & .00 & 6 \\
\hline 3466030 & 358631 & 2.50 & 50.00 & 1 \\
\hline 3466031 & 358987 & 4.50 & 20.00 & 5 \\
\hline 3466153 & 358212 & 4.50 & 10.00 & 1 \\
\hline 3466181 & 358986 & 2.50 & 10.00 & 5 \\
\hline
\end{tabular}


Table G-1. continued...

\begin{tabular}{|c|c|c|c|c|}
\hline UTM Northing & UTM Easting & Length $(\mathrm{cm})$ & Cortex (\%) & Artifact Tvpe \\
\hline 3466227 & 358995 & 4.50 & 10.00 & 1 \\
\hline 3466794 & 352031 & 8.50 & 80.00 & 1 \\
\hline 3466800 & 351185 & 2.50 & 50.00 & 1 \\
\hline 3466827 & 351165 & 11.50 & 30.00 & 2 \\
\hline 3466832 & 351154 & 7.50 & 90.00 & 1 \\
\hline 3466836 & 351148 & 15.50 & 80.00 & 2 \\
\hline 3466850 & 349632 & 8.50 & & 6 \\
\hline 3466936 & 352169 & 2.50 & .00 & 5 \\
\hline 3466958 & 351223 & 2.50 & 40.00 & 1 \\
\hline 3467003 & 351066 & 5.50 & 50.00 & 2 \\
\hline 3467092 & 351226 & 1.50 & 30.00 & 1 \\
\hline 3467093 & 351195 & 6.50 & 50.00 & 5 \\
\hline 3467094 & 351217 & 4.50 & 50.00 & 1 \\
\hline 3467099 & 351217 & 10.50 & & 2 \\
\hline 3467102 & 351237 & 5.50 & 10.00 & 1 \\
\hline 3467103 & 350949 & 3.50 & .00 & 1 \\
\hline 3467115 & 351220 & 6.50 & 10.00 & 1 \\
\hline 3467149 & 351207 & 7.50 & & 2 \\
\hline 3467242 & 351103 & 4.50 & 50.00 & 1 \\
\hline 3467243 & 351103 & 2.50 & & 1 \\
\hline 3467277 & 350709 & 2.50 & & 6 \\
\hline 3467283 & 351716 & 11.50 & 70.00 & 2 \\
\hline 3467295 & 351280 & 8.50 & 10.00 & 6 \\
\hline 3467295 & 351280 & 8.50 & 10.00 & 6 \\
\hline 3467298 & 350734 & 4.50 & 10.00 & 1 \\
\hline 3467372 & 351350 & 8.50 & 40.00 & 1 \\
\hline 3467372 & 351350 & 8.50 & 40.00 & 1 \\
\hline 3467376 & 350947 & 4.50 & 60.00 & 2 \\
\hline 3467378 & 350959 & 6.50 & 20.00 & 1 \\
\hline 3467380 & 350961 & 4.50 & .00 & 1 \\
\hline 3467388 & 351352 & 4.50 & 10.00 & 1 \\
\hline 3467388 & 351352 & 4.50 & 10.00 & 1 \\
\hline 3467389 & 350974 & 7.50 & 50.00 & 2 \\
\hline 3467396 & 351341 & 4.50 & .00 & 1 \\
\hline 3467396 & 351341 & 4.50 & .00 & 1 \\
\hline 3467398 & 351313 & 2.50 & & 1 \\
\hline 3467398 & 351313 & 2.50 & & 1 \\
\hline 3467405 & 351652 & 12.50 & 70.00 & 2 \\
\hline 3467460 & 350226 & 4.50 & .00 & 1 \\
\hline 3467465 & 350088 & 4.50 & .00 & 1 \\
\hline 3467477 & 351251 & 5.50 & 20.00 & 6 \\
\hline 3467502 & 350226 & 4.50 & .00 & 1 \\
\hline 3467513 & 350695 & 4.50 & .00 & 1 \\
\hline 3467514 & 350695 & 3.50 & .00 & 1 \\
\hline 3467536 & 349646 & 3.50 & .00 & 1 \\
\hline 3467537 & 349637 & 4.50 & .00 & 2 \\
\hline 3467555 & 350289 & 3.50 & .00 & 4 \\
\hline 3467567 & 353926 & 1.50 & 10.00 & 1 \\
\hline 3467568 & 353931 & 1.50 & & 1 \\
\hline 3467601 & 349670 & 5.50 & & 1 \\
\hline 3467636 & 351737 & 5.50 & 20.00 & 1 \\
\hline 3467641 & 351738 & 3.50 & & 1 \\
\hline
\end{tabular}


Table G-1. continued...

\begin{tabular}{|c|c|c|c|c|}
\hline UTM Northing & UTM Easting & Length $(\mathrm{cm})$ & Cortex (\%) & Artifact Tvpe \\
\hline 3467681 & 351213 & & .00 & 1 \\
\hline 3467682 & 351248 & 6.50 & 20.00 & 1 \\
\hline 3467704 & 351769 & 9.50 & .00 & 4 \\
\hline 3467790 & 352543 & 4.50 & 30.00 & 1 \\
\hline 3467809 & 352580 & 3.50 & 30.00 & 1 \\
\hline 3467815 & 351953 & 5.50 & & 6 \\
\hline 3467817 & 352842 & 3.50 & .00 & 1 \\
\hline 3467819 & 351976 & 3.50 & & 6 \\
\hline 3467834 & 349019 & 3.50 & & 1 \\
\hline 3467840 & 352572 & 9.50 & 10.00 & 2 \\
\hline 3467842 & 348887 & 1.50 & .00 & 1 \\
\hline 3467845 & 348887 & 2.50 & 10.00 & 1 \\
\hline 3467855 & 352471 & 7.50 & .00 & 4 \\
\hline 3467872 & 348923 & 6.50 & 10.00 & 1 \\
\hline 3467881 & 353678 & 1.50 & & 1 \\
\hline 3467930 & 352591 & 5.50 & .00 & 1 \\
\hline 3467947 & 352606 & 5.50 & 40.00 & 1 \\
\hline 3467975 & 352243 & 4.50 & 20.00 & 1 \\
\hline 3467986 & 352281 & 3.50 & .00 & 1 \\
\hline 3467987 & 352282 & 4.50 & .00 & 1 \\
\hline 3467989 & 352278 & 2.50 & .00 & 1 \\
\hline 3467989 & 352278 & 1.50 & & 1 \\
\hline 3467989 & 352283 & 6.50 & 10.00 & 1 \\
\hline 3467989 & 352283 & 5.50 & .00 & 5 \\
\hline 3467996 & 352627 & 12.50 & 20.00 & 2 \\
\hline 3467998 & 352627 & 4.50 & 40.00 & 1 \\
\hline 3468007 & 353599 & 4.50 & .00 & 6 \\
\hline 3468035 & 352604 & 16.50 & 20.00 & 2 \\
\hline 3468038 & 353575 & 5.50 & .00 & 1 \\
\hline 3468060 & 352457 & 7.50 & & 6 \\
\hline 3468077 & 352607 & 7.50 & 20.00 & 2 \\
\hline 3468082 & 352607 & 8.50 & 10.00 & 2 \\
\hline 3468095 & 352462 & 1.50 & & 1 \\
\hline 3468102 & 352463 & 9.50 & 50.00 & 2 \\
\hline 3468107 & 353534 & 2.50 & 70.00 & 1 \\
\hline 3468157 & 352484 & 8.50 & 10.00 & 1 \\
\hline 3468185 & 350883 & 3.50 & 30.00 & 1 \\
\hline 3468186 & 350883 & 6.50 & 10.00 & 1 \\
\hline 3468275 & 352446 & 7.50 & 70.00 & 5 \\
\hline 3468401 & 354348 & 4.50 & & 6 \\
\hline 3468402 & 353457 & 6.50 & .00 & 5 \\
\hline 3468528 & 352486 & 4.50 & .00 & 1 \\
\hline 3468529 & 352488 & 5.50 & .00 & 1 \\
\hline 3468730 & 352486 & 9.50 & 20.00 & 5 \\
\hline 3468749 & 353769 & 5.50 & 20.00 & 4 \\
\hline 3468969 & 352567 & 5.50 & 50.00 & 1 \\
\hline 3469021 & 353000 & 7.50 & 10.00 & 1 \\
\hline 3469268 & 354216 & 2.50 & .00 & 5 \\
\hline 3469270 & 354072 & 1.50 & .00 & 1 \\
\hline 3469286 & 354083 & 2.50 & .00 & 6 \\
\hline 3469293 & 354082 & 2.50 & .00 & 6 \\
\hline 3469324 & 354104 & 2.50 & & 1 \\
\hline
\end{tabular}


Table G-1. continued...

\begin{tabular}{|c|c|c|c|c|}
\hline UTM Northing & UTM Easting & Length $(\mathrm{cm})$ & Cortex (\%) & Artifact Type \\
\hline 3469695 & 354341 & 2.50 & 10.00 & 1 \\
\hline 3469817 & 349623 & & .00 & 5 \\
\hline 3469819 & 349666 & 4.50 & 50.00 & 1 \\
\hline 3469825 & 350154 & 3.50 & 10.00 & 1 \\
\hline 3469830 & 350148 & 4.50 & .00 & 1 \\
\hline 3469833 & 350152 & 2.50 & .00 & 1 \\
\hline 3469857 & 352993 & 5.50 & 40.00 & 2 \\
\hline 3469865 & 350012 & 3.50 & 10.00 & 1 \\
\hline 3469880 & 350111 & 6.50 & 20.00 & 1 \\
\hline 3469884 & 349272 & 2.50 & 20.00 & 1 \\
\hline 3469892 & 354221 & & & 10 \\
\hline 3469907 & 349977 & & .00 & 5 \\
\hline 3469961 & 348147 & 4.50 & 20.00 & 1 \\
\hline 3469962 & 350262 & 5.50 & 20.00 & 1 \\
\hline 3469963 & 350260 & 2.50 & & 1 \\
\hline 3469994 & 348124 & 3.50 & .00 & 1 \\
\hline 3470106 & 348193 & 7.50 & .00 & 6 \\
\hline 3470173 & 356026 & 2.50 & 20.00 & 1 \\
\hline 3470183 & 356014 & 3.50 & .00 & 5 \\
\hline 3470205 & 356030 & 1.50 & .00 & 4 \\
\hline 3470271 & 348256 & 6.50 & .00 & 5 \\
\hline 3470273 & 355697 & 4.50 & 10.00 & 4 \\
\hline 3470277 & 355699 & 2.50 & .00 & 1 \\
\hline 3470282 & 355691 & 2.50 & 10.00 & 1 \\
\hline 3470284 & 355693 & 2.50 & .00 & 1 \\
\hline 3470337 & 350715 & 5.50 & 20.00 & 6 \\
\hline 3470526 & 356120 & 6.50 & 70.00 & 2 \\
\hline 3470597 & 351008 & 6.50 & 20.00 & 1 \\
\hline 3470676 & 352075 & 6.50 & 20.00 & 4 \\
\hline 3470700 & 353770 & 1.50 & 10.00 & 1 \\
\hline 3470702 & 353768 & 4.50 & 50.00 & 1 \\
\hline 3470716 & 353617 & 7.50 & 10.00 & 2 \\
\hline 3470717 & 353616 & 2.50 & .00 & 1 \\
\hline 3470721 & 350671 & 7.50 & 20.00 & 1 \\
\hline 3470742 & 353700 & 4.50 & 40.00 & 2 \\
\hline 3470780 & 350783 & 9.50 & 10.00 & 2 \\
\hline 3470789 & 349741 & 5.50 & .00 & 1 \\
\hline 3470802 & 349719 & 3.50 & 20.00 & 1 \\
\hline 3470818 & 352674 & 9.50 & & 2 \\
\hline 3470827 & 352225 & 4.50 & 20.00 & 1 \\
\hline 3470856 & 352944 & 5.50 & 40.00 & 1 \\
\hline 3470875 & 353075 & 4.50 & .00 & 6 \\
\hline 3470941 & 350060 & 1.50 & 10.00 & 1 \\
\hline 3470971 & 350060 & 4.50 & 10.00 & 1 \\
\hline 3471076 & 356942 & 10.50 & 40.00 & 2 \\
\hline 3471089 & 356854 & 2.50 & .00 & 4 \\
\hline 3471277 & 348955 & 6.50 & .00 & 1 \\
\hline 3471299 & 356839 & & & 10 \\
\hline 3471433 & 350579 & 5.50 & .00 & 1 \\
\hline 3471598 & 354618 & 7.50 & 10.00 & 1 \\
\hline 3471810 & 354447 & 4.50 & & 1 \\
\hline 3471814 & 354401 & 9.50 & 60.00 & 5 \\
\hline
\end{tabular}


Table G-1. continued...

\begin{tabular}{|c|c|c|c|c|}
\hline UTM Northing & UTM Easting & Length $(\mathrm{cm})$ & Cortex (\%) & Artifact Type \\
\hline 3471821 & 354406 & 6.50 & .00 & 4 \\
\hline 3471899 & 352552 & 7.50 & .00 & 1 \\
\hline 3471903 & 352550 & 11.50 & .00 & 4 \\
\hline 3471932 & 351983 & 4.50 & 20.00 & 1 \\
\hline 3471941 & 350037 & 4.50 & 80.00 & 1 \\
\hline 3471941 & 350040 & 4.50 & 90.00 & 1 \\
\hline 3471950 & 351988 & 3.50 & 50.00 & 1 \\
\hline 3471956 & 350023 & 6.50 & 80.00 & 1 \\
\hline 3471966 & 346666 & 3.50 & .00 & 1 \\
\hline 3471967 & 350929 & 3.50 & 50.00 & 1 \\
\hline 3472068 & 351539 & 6.50 & .00 & 2 \\
\hline 3472078 & 352287 & 4.50 & 10.00 & 1 \\
\hline 3472116 & 351536 & 6.50 & .00 & 2 \\
\hline 3472171 & 348383 & 1.50 & .00 & 1 \\
\hline 3472192 & 347317 & 7.50 & .00 & 6 \\
\hline 3472321 & 347592 & 2.50 & 20.00 & 1 \\
\hline 3472333 & 347533 & 6.50 & 20.00 & 6 \\
\hline 3472333 & 347592 & 3.50 & .00 & 2 \\
\hline 3472338 & 347580 & 3.50 & .00 & 1 \\
\hline 3472352 & 347577 & 3.50 & .00 & 1 \\
\hline 3472382 & 347537 & 2.50 & 30.00 & 2 \\
\hline 3472384 & 347573 & 6.50 & 10.00 & 1 \\
\hline 3472388 & 347545 & 5.50 & .00 & 1 \\
\hline 3472390 & 347541 & 4.50 & 70.00 & 2 \\
\hline 3472400 & 347539 & 4.50 & .00 & 1 \\
\hline 3472453 & 354197 & 4.50 & 50.00 & 1 \\
\hline 3472473 & 346665 & 9.50 & 30.00 & 2 \\
\hline 3472497 & 352509 & 5.50 & .00 & 2 \\
\hline 3472552 & 354092 & 5.50 & .00 & 1 \\
\hline 3472565 & 352138 & 6.50 & 20.00 & 2 \\
\hline 3472586 & 352520 & 11.50 & 20.00 & 2 \\
\hline 3472799 & 352113 & 7.50 & 40.00 & 6 \\
\hline 3472844 & 353577 & 6.50 & .00 & 4 \\
\hline 3472847 & 353107 & 3.50 & .00 & 1 \\
\hline 3472851 & 352472 & 2.50 & .00 & 1 \\
\hline 3472855 & 352474 & 2.50 & .00 & 1 \\
\hline 3472858 & 353203 & 4.50 & .00 & 1 \\
\hline 3472873 & 353147 & 1.50 & .00 & 1 \\
\hline 3472876 & 353552 & 5.50 & .00 & 1 \\
\hline 3472880 & 353595 & 3.50 & .00 & 1 \\
\hline 3472903 & 343059 & 2.50 & .00 & 1 \\
\hline 3472903 & 343059 & 4.50 & .00 & 1 \\
\hline 3472903 & 353059 & 8.50 & 40.00 & 1 \\
\hline 3472932 & 352520 & 8.50 & 30.00 & 1 \\
\hline 3472940 & 352214 & 2.50 & .00 & 1 \\
\hline 3472951 & 352430 & 4.50 & .00 & 1 \\
\hline 3473058 & 346735 & 3.50 & 10.00 & 1 \\
\hline 3473058 & 346735 & 2.50 & & 1 \\
\hline 3473089 & 346949 & 3.50 & & 1 \\
\hline 3473114 & 346954 & 5.50 & & 1 \\
\hline 3473125 & 347025 & 12.50 & 10.00 & 2 \\
\hline 3473134 & 346752 & 10.50 & 70.00 & 6 \\
\hline
\end{tabular}


Table G-1. continued...

\begin{tabular}{|c|c|c|c|c|}
\hline UTM Northing & UTM Easting & Length $(\mathrm{cm})$ & Cortex (\%) & Artifact Type \\
\hline 3473136 & 352685 & 9.50 & 10.00 & 2 \\
\hline 3473150 & 352684 & 5.50 & 10.00 & 1 \\
\hline 3473173 & 346736 & 2.50 & 20.00 & 1 \\
\hline 3473177 & 346739 & 2.50 & 20.00 & 1 \\
\hline 3473222 & 346741 & 3.50 & 10.00 & 1 \\
\hline 3473222 & 346742 & 1.50 & & 1 \\
\hline 3473222 & 346743 & 1.50 & & 1 \\
\hline 3473233 & 352695 & 6.50 & 30.00 & 1 \\
\hline 3473253 & 346743 & 4.50 & 20.00 & 1 \\
\hline 3473263 & 352679 & 6.50 & .00 & 1 \\
\hline 3473338 & 346750 & 2.50 & & 1 \\
\hline 3473365 & 352680 & 6.50 & 10.00 & 1 \\
\hline 3473374 & 346963 & 6.50 & 90.00 & 2 \\
\hline 3473395 & 354210 & 3.50 & & 6 \\
\hline 3473411 & 346731 & 2.50 & 10.00 & 1 \\
\hline 3473415 & 354212 & 8.50 & & 1 \\
\hline 3473421 & 354197 & 5.50 & 10.00 & 6 \\
\hline 3473423 & 346913 & 5.50 & 20.00 & 1 \\
\hline 3473423 & 346961 & 6.50 & & 1 \\
\hline 3473430 & 346722 & 6.50 & 10.00 & 1 \\
\hline 3473433 & 354184 & 4.50 & 20.00 & 1 \\
\hline 3473434 & 354188 & 2.50 & .00 & 1 \\
\hline 3473449 & 354185 & 4.50 & .00 & 6 \\
\hline 3473452 & 346827 & 8.50 & 20.00 & 2 \\
\hline 3473453 & 354190 & 8.50 & 10.00 & 1 \\
\hline 3473500 & 346771 & 3.50 & .00 & 4 \\
\hline 3473515 & 352107 & 7.50 & 10.00 & 1 \\
\hline 3473515 & 354438 & 7.50 & 10.00 & 1 \\
\hline 3473518 & 354418 & 6.50 & & 2 \\
\hline 3473566 & 352411 & 2.50 & .00 & 1 \\
\hline 3473651 & 353282 & 6.50 & 10.00 & 1 \\
\hline 3473653 & 353062 & 3.50 & 40.00 & 1 \\
\hline 3473719 & 354134 & 3.50 & 10.00 & 6 \\
\hline 3473727 & 353401 & 6.50 & 50.00 & 4 \\
\hline 3473731 & 346742 & 1.50 & .00 & 1 \\
\hline 3473744 & 354422 & & & 10 \\
\hline 3473838 & 352619 & 3.50 & .00 & 1 \\
\hline 3473857 & 352599 & 4.50 & 10.00 & 1 \\
\hline 3473915 & 353599 & 3.50 & .00 & 1 \\
\hline 3473920 & 353742 & 3.50 & .00 & 5 \\
\hline 3473930 & 352060 & & & 10 \\
\hline 3473943 & 352044 & & & 10 \\
\hline 3473984 & 353699 & 5.50 & .00 & 1 \\
\hline 3473988 & 353637 & 4.50 & .00 & 1 \\
\hline 3473994 & 353098 & 3.50 & .00 & 1 \\
\hline 3474003 & 354176 & 7.50 & 50.00 & 5 \\
\hline 3474009 & 353945 & 3.50 & 20.00 & 1 \\
\hline 3474019 & 352018 & 5.50 & 10.00 & 6 \\
\hline 3474025 & 353091 & 1.50 & 50.00 & 1 \\
\hline 3474025 & 354158 & 4.50 & 50.00 & 1 \\
\hline 3474026 & 353246 & 4.50 & .00 & 1 \\
\hline 3474026 & 353668 & 3.50 & 20.00 & 1 \\
\hline
\end{tabular}


Table G-1. continued...

\begin{tabular}{|c|c|c|c|c|}
\hline UTM Northing & UTM Easting & Length $(\mathrm{cm})$ & Cortex (\%) & Artifact Type \\
\hline 3474027 & 354137 & 3.50 & 10.00 & 1 \\
\hline 3474035 & 354135 & 6.50 & 40.00 & 1 \\
\hline 3474047 & 353627 & 1.50 & .00 & 1 \\
\hline 3474047 & 353627 & 2.50 & .00 & 1 \\
\hline 3474048 & 352180 & 1.50 & 50.00 & 1 \\
\hline 3474048 & 352180 & 3.50 & 50.00 & 1 \\
\hline 3474050 & 353566 & 2.50 & 50.00 & 1 \\
\hline 3474059 & 353076 & 7.50 & .00 & 2 \\
\hline 3474061 & 352088 & 3.50 & .00 & 1 \\
\hline 3474073 & 352176 & 4.50 & 50.00 & 1 \\
\hline 3474080 & 352283 & 1.50 & .00 & 1 \\
\hline 3474084 & 352217 & 3.50 & 20.00 & 2 \\
\hline 3474092 & 353539 & 2.50 & .00 & 1 \\
\hline 3474093 & 352271 & 3.50 & .00 & 1 \\
\hline 3474094 & 352397 & 3.50 & 20.00 & 1 \\
\hline 3474097 & 352569 & 14.50 & 10.00 & 1 \\
\hline 3474101 & 351969 & 6.50 & .00 & 1 \\
\hline 3474105 & 352049 & 4.50 & .00 & 1 \\
\hline 3474123 & 352486 & 5.50 & 10.00 & 5 \\
\hline 3474126 & 351946 & 3.50 & .00 & 1 \\
\hline 3474126 & 353200 & 3.50 & .00 & 1 \\
\hline 3474152 & 352990 & 3.50 & 10.00 & 1 \\
\hline 3474160 & 351843 & 2.50 & .00 & 5 \\
\hline 3474161 & 353029 & 2.50 & .00 & 1 \\
\hline 3474167 & 353499 & 1.50 & .00 & 1 \\
\hline 3474170 & 352347 & 5.50 & .00 & 5 \\
\hline 3474195 & 353262 & 5.50 & 10.00 & 2 \\
\hline 3474197 & 352418 & 1.50 & .00 & 1 \\
\hline 3474199 & 351893 & 1.50 & .00 & 1 \\
\hline 3474206 & 351862 & 1.50 & .00 & 1 \\
\hline 3474227 & 351757 & 3.50 & .00 & 1 \\
\hline 3474260 & 351765 & 6.50 & 10.00 & 6 \\
\hline 3474267 & 353002 & 6.50 & 10.00 & 1 \\
\hline 3474274 & 352962 & 2.50 & .00 & 1 \\
\hline 3474276 & 353033 & 17.50 & 60.00 & 2 \\
\hline 3474286 & 351742 & 2.50 & 10.00 & 1 \\
\hline 3474286 & 353177 & 3.50 & .00 & 1 \\
\hline 3474288 & 352985 & 2.50 & 20.00 & 1 \\
\hline 3474299 & 353159 & 2.50 & .00 & 1 \\
\hline 3474323 & 357421 & 2.50 & 20.00 & 1 \\
\hline 3474344 & 351873 & 4.50 & 50.00 & 1 \\
\hline 3474345 & 353090 & 5.50 & .00 & 1 \\
\hline 3474349 & 352862 & 2.50 & 30.00 & 1 \\
\hline 3474350 & 351850 & 8.50 & 10.00 & 1 \\
\hline 3474354 & 352895 & 9.50 & .00 & 2 \\
\hline 3474356 & 353404 & 4.50 & 20.00 & 1 \\
\hline 3474369 & 352515 & 1.50 & .00 & 1 \\
\hline 3474379 & 352587 & 2.50 & .00 & 1 \\
\hline 3474408 & 352337 & 5.50 & 10.00 & 1 \\
\hline 3474416 & 351578 & 2.50 & 50.00 & 2 \\
\hline 3474416 & 353014 & 4.50 & & 1 \\
\hline 3474416 & 353014 & 5.50 & & 1 \\
\hline
\end{tabular}


Table G-1. continued...

\begin{tabular}{|c|c|c|c|c|}
\hline UTM Northing & UTM Easting & Length (cm) & Cortex $(\%)$ & Artifact Type \\
\hline 3474416 & 353014 & 9.50 & & 1 \\
\hline 3474423 & 352328 & 2.50 & .00 & 1 \\
\hline 3474434 & 352908 & 7.50 & 10.00 & 5 \\
\hline 3474461 & 351666 & 2.50 & 10.00 & 1 \\
\hline 3474492 & 351598 & 1.50 & .00 & 1 \\
\hline 3474492 & 351598 & 1.50 & 10.00 & 1 \\
\hline 3474511 & 353436 & 2.50 & .00 & 1 \\
\hline 3474523 & 351520 & 3.50 & .00 & 1 \\
\hline 3474557 & 353573 & 5.50 & 20.00 & 1 \\
\hline 3474579 & 352889 & 99.00 & 20.00 & 2 \\
\hline 3474591 & 351530 & 4.50 & .00 & 1 \\
\hline 3474595 & 353525 & 1.50 & & 1 \\
\hline 3474602 & 353528 & 4.50 & & 1 \\
\hline 3474610 & 351931 & 8.50 & 80.00 & 2 \\
\hline 3474644 & 351650 & 3.50 & .00 & 1 \\
\hline 3474686 & 351603 & 1.50 & .00 & 1 \\
\hline 3474721 & 351520 & 2.50 & .00 & 1 \\
\hline 3474746 & 353455 & 5.50 & 10.00 & 1 \\
\hline 3474755 & 351625 & 2.50 & .00 & 1 \\
\hline 3474755 & 353319 & 4.50 & & 1 \\
\hline 3474759 & 353327 & 4.50 & 50.00 & 6 \\
\hline 3474759 & 353330 & 2.50 & & 1 \\
\hline 3474763 & 353332 & 3.50 & 10.00 & 1 \\
\hline 3474765 & 352647 & & & 10 \\
\hline 3474797 & 351622 & 5.50 & .00 & 1 \\
\hline 3474818 & 351824 & 8.50 & .00 & 2 \\
\hline 3474822 & 351889 & 5.50 & 10.00 & 4 \\
\hline 3475828 & 353620 & 9.50 & 30.00 & 6 \\
\hline
\end{tabular}




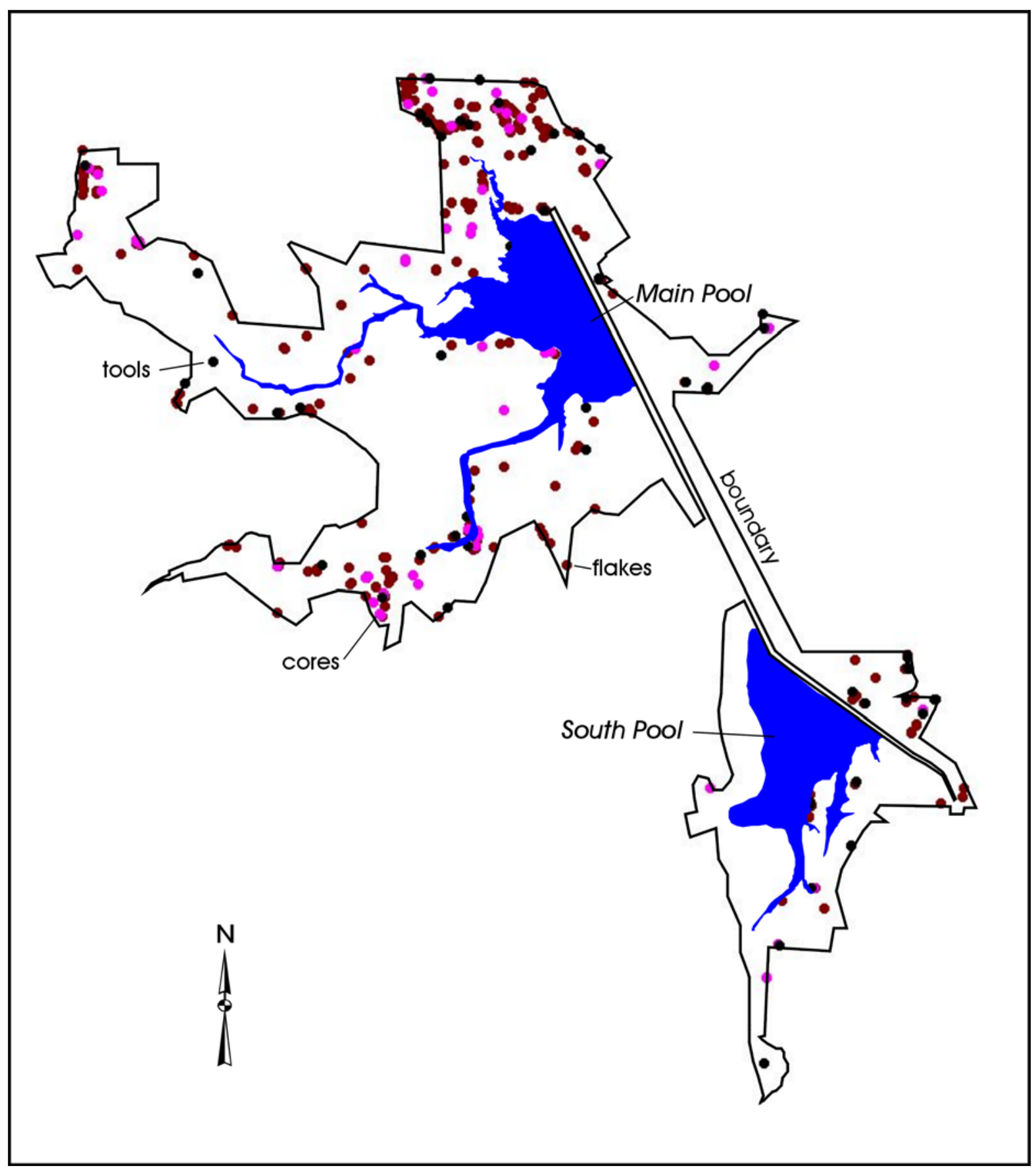

Figure G-1. Distribution of Isolated Finds recorded during Twin Buttes Archaeological Project. 


\section{Appendix H: \\ Twin Buttes Archaeological Project}

Radiocarbon Results 


\section{REPORT OF RADIOCARBON DATING ANALYSES}

Dr. Raymond Mauldin

University of Texas at San Antonio

\begin{tabular}{lccc} 
Sample Data & $\begin{array}{c}\text { Measured } \\
\text { Radiocarbon Age }\end{array}$ & $\begin{array}{c}{ }^{2} \mathrm{C} /{ }^{2} \mathrm{C} \\
\text { Ratio }\end{array}$ & $\begin{array}{c}\text { Conventional } \\
\text { Radiocarbon Age (*) }\end{array}$ \\
\hline Beta-136034 & $480+1-40 \mathrm{BP}$ & $-26.70 \% 0$ & $460+-40 \mathrm{BP}$
\end{tabular}

SAMPLE \#: FS $+52-9$

ANALYSIS: Standard-AMS

MATERIAL/PRETREATMENT:(chatred materialy: acid/alkali/acid

Beta-136035

$870+-40 \mathrm{BP}$

$-25.40 \% 00$

$870+1-40 \mathrm{BP}$
Report Date: December 4, 1999

Material Received: November 4, 1999

SAMPLE \#: FS\#1 52-2

ANALYSIS: Standard-AMS

MATERLAL/PRETREATMENT (charred material): acid/alkali/acid

NOTE: It is important to read the calendar calibration information and to use the calendar

calibrated results (reported separately) when interpreting these results in $\mathrm{AD} / \mathrm{BC}$ terms.

Dates are reported as RCYBP (radiocarbon years before present, "present" = 1950A.D.). By International convention, the modern reference standard was $95 \%$ of the $\mathrm{C} 14$ content of the National Bureau of Standards' Oxalic Acid \& calculated using the Libby C14 half life (5568 years). Quoted errors represent 1 standard deviation statistics ( $68 \%$ probability) \& are based on combined measurements of the sample, background, and modern reference standards.

\begin{abstract}
Measured $\mathrm{C} 13 / \mathrm{C} 12$ ratios were calculated relative to the PDB-1 international standard and the RCYBP ages were normalized to -25 per mil. If the ratio and age are accompanied by an $\left({ }^{*}\right)$, then the C13/C12 value was estimated, based on values typical of the material type. The quoted results are NOT calibrated to calendar years. Calibration to calendar years should be calculated using the Conventional $\mathrm{C} 14$ age.
\end{abstract}


CALIBRATION OF RADIOCARBON AGE TO CALENDAR YEARS

(Variables: C13/C12=-26.7:lab. mult=1)

Laboratory number: Beta-136034

Conventional radiocarbon age: $\quad 460 \pm 40 \mathrm{BP}$

2 Sigma calibrated result: Cal AD 1410 to 1480 (Cal BP 540 to 470) (95\% probability)

Intercept data

Intercept of radiocarbon age

with calibration curve:

1 Sigma calibrated result: $\mathrm{Cal}$ AD 1425 to 1450 (Cal BP 525 to 500 )

(68\% probability)

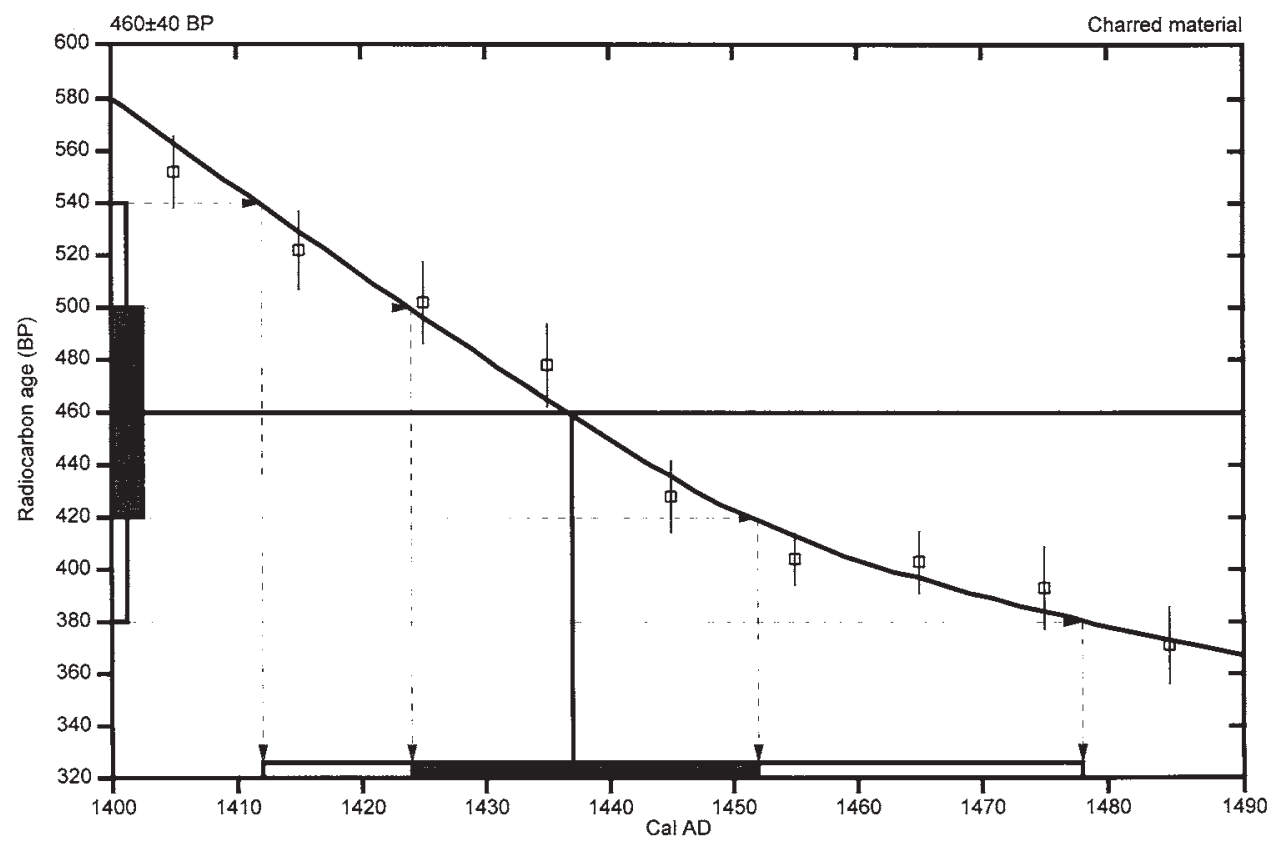

References:

Database used

Calibration Databas

Editorial Comment

Editorial Comment
Stuiver, $M$., van der Plicht, H., 1998, Radiocarbon 40(3), pxii-xiii

INTCAL98 Radiocarbon Age Calibration

Stuiver, M. et. al., 1998, Radiocarbon 40(3), p1041-1083

Mathematics

A Simplified Approach to Calibrating C14 Dates

Talma, A. S., Vogel, J. C., 1993, Radiocarbon 35(2), p317-322

Beta Analytic Radiocarbon Dating Laboratory

4985 S.W.74th Court, Miami, Florida 33155 • Tel: (305)667-5167 • Fax: (305)663-0964 • E-mail: beta@radiocarbon.com 


\section{CALIBRATION OF RADIOCARBON AGE TO CALENDAR YEARS}

(Variables: C13/C12=-25.4:lab. mult=1)

Laboratory number: Beta-136035

Conventional radiocarbon age: $870 \pm 40 \mathrm{BP}$

2 Sigma calibrated result: Cal AD 1040 to 1255 (Cal BP 910 to 695)

(95\% probability)

Intercept data

Intercept of radiocarbon age

with calibration curve: Cal AD 1185 (Cal BP 765)

1 Sigma calibrated result: Cal AD 1155 to 1220 (Cal BP 795 to 730 )

(68\% probability)

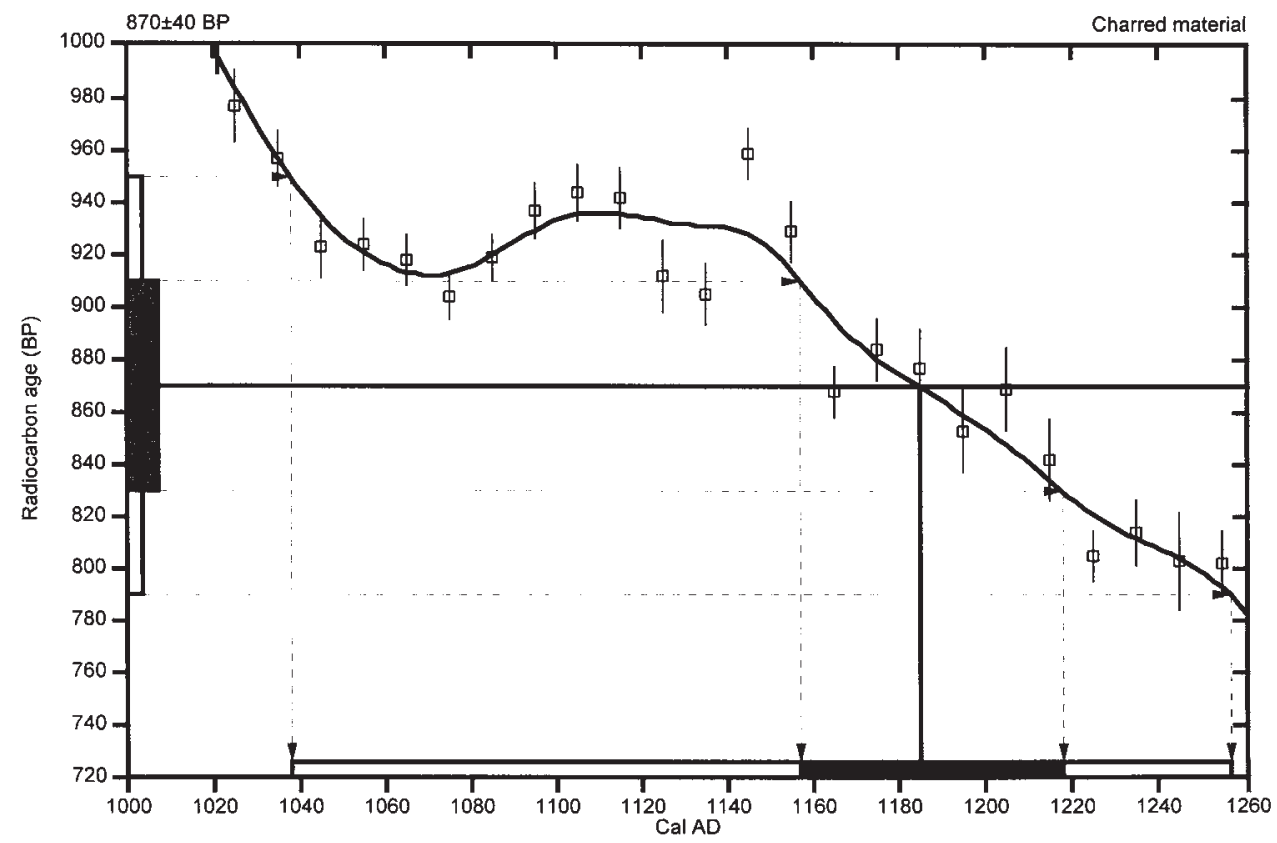

References:

Database used
INTCAL98

Calibration Database

Editorial Comment

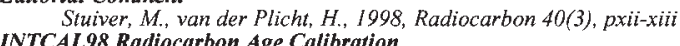

CAL98 Radiocarbon Age Calibration

Stuiver, M., et. al., 1998, Radiocarbon 40(3), p1041-1083

Mathematics

A Simplified Approach to Calibrating C14 Dates

Talma, A. S., Vogel, J. C., 1993, Radiocarbon 35(2), p317-322

\section{Beta Analytic Radiocarbon Dating Laboratory}

4985 S.W.74th Court, Miami, Florida 33155.Tel: (305)667-5167•Fax: (305)663-0964·E-mail: beta@radiocarbon.com 
BETA ANALYTIC INC.

DR. M.A. TAMERS and MR. D.G. HOOD
UNIVERSITY BRANCH

4985 S.W. 74 COURT

MIAMI, FLORIDA, USA 33155

PH: 305/667-5167 FAX: 305/663-0964

E-MAIL: beta@radiocarbon.com

\section{REPORT OF RADIOCARBON DATING ANALYSES}

Dr. Raymond Mauldin/Lee Nordt

University of Texas at Sen Antonio
Report Dete: December 23, 1999

Material Received: November 15, 1999

\begin{tabular}{rlcc}
\hline Sample Data & $\begin{array}{c}\text { Moesured } \\
\text { Radiocarbon Age }\end{array}$ & $\begin{array}{c}{ }^{13} \mathrm{C} /{ }^{12} \mathrm{C} \\
\text { Ratio }\end{array}$ & $\begin{array}{c}\text { Conventional } \\
\text { Radiocarbon Age (*) }\end{array}$ \\
\hline Beta-136274 & $9220+/-70 \mathrm{BP}$ & $-22.10 / 00$ & $9260+/-70 \mathrm{BP}$
\end{tabular}

SAMPLE \#. BHT2BK1 b2

ANALYSIS: Standard-AMS

MATERIAL/PRETREATMENT:(organic sediment): acid washes

Beta- 136276

$3200+/-50 \mathrm{BP}$

$-15.0 \mathrm{o} / \mathrm{o}$

$3360+/-50 \mathrm{BP}$

SAMPLE \#: BHT6AKb1

ANAL YSIS: Standard-AMS

MATERIAL/PRETREATMENT:(onganic sediment): acid washes

Beta-136277

$780+/-60 \mathrm{BP}$

$-17.5 \circ / 00$

$910+/-60 \mathrm{BP}$

SAMPLE \#: CBIbC2b2

ANALYSIS: radiometric-standard

MATERIAL/PRETREATMENT:(organic sediment): acid washes

COMMENT: low carbon sediment requiring special handling

\begin{tabular}{lll}
\hline Beta-136278 & $5740+/-70 \mathrm{BP}$ & $-23.6 \%$
\end{tabular}

SAMPLE \#: CB2acB2

ANALYSIS: Standard-AMS

MATERIAL/PRETREATMENT:(organic sediment): acid washes

Beta-136279

$3360+1-60 \mathrm{BP}$

$-18.80 / 00$

$3460+/-60 \mathrm{BP}$

SAMPLE \#: CB5C5b2

ANALYSIS: Standard-AMS

MATERIAL/PRETREATMENT:(organic sediment): acid washes

Dates are reported as RCYBP (radiocarbon years before present, "present" = 1950A.D.). By International convention, the modern reference standard was $95 \%$ of the C14 content of the National Bureau of Standards' Oxalic Acid \& calculated using the Libby C14 half life ( 5568 years). Quoted errors represent 1 standard deviation statistics ( $68 \%$ probability) \& are based on combined measurements of the sample, background, and modern reference standards.
Measured $\mathrm{C} 13 / \mathrm{C} 12$ ratios were calculated relative to the PDB- 1 international standard and the RCYBP ages were normalized to -25 per mil. If the ratio and age are accompanied by an (*), then the $\mathrm{C} 13 / \mathrm{C} 12$ value was estimated, based on values typical of the material type. The quoted results are NOT calibrated to calendar years. Calibration to calendar years should be calculated using the Conventional C14 age. 


\section{REPORT OF RADIOCARBON DATING ANALYSES}

Dr. Raymond Mauldin/Lee Nordt
Page 2 of 2

Conventional

Radiocarbon Ago (")

$3440+/-50 \mathrm{BP}$

Beta-136280

$3340+/-50 \mathrm{BP}$

$-18.4 \%$

${ }_{\text {Ratio }} \mathrm{C} /{ }^{12} \mathrm{C}$

SAMPLE \#: CB7Cb2

ANAL YSIS: Standard-AMS

MATERIAL/PRETREATMENT:(organic sediment): acid washes

NOTE: It is important to read the calendar calibration information and to use the calendar calibrated results (reported separately) when interpreting these results in $\mathrm{AD} / \mathrm{BC}$ terms.

NOTE: Sample BHT4Ab2 was submitted but not analyzed.

Dates are reported as RCYBP (radiocarbon years before present, "present" = 1950A.D.). By International convention, the modern reference standard was $95 \%$ of the C14 content of the National Bureau of Standards' Oxalic Acid \& calculated using the Libby C14 half life ( 5568 years). Quoted errors represent 1 standard deviation statistics ( $68 \%$ probability) \& are based on combined measurements of the sample, background, and modern reference standards.
Measured $\mathrm{C} 13 / \mathrm{C} 12$ ratios were calculated relative to the PDB-1 international standard and the RCYBP ages were normalized to -25 per mil. If the ratio and age are accompanied by an $\left({ }^{*}\right)$, then the C13/C12 value was estimated, based on values typical of the material type. The quoted results are NOT calibrated to calendar years. Calibration to calendar years should be calculated using the Conventional C14 age. 


\section{CALIBRATION OF RADIOCARBON AGE TO CALENDAR YEARS}

(Variables: $\mathrm{C} 13 / \mathrm{C} 12=-22.1:$ lab. mult=1)

Laboratory number: Beta-136274

Conventional radiocarbon age: $9260 \pm 70 \mathrm{BP}$

2 Sigma calibrated result: Cal BC 8640 to 8285 (Cal BP 10590 to 10235) (95\% probability)

Intercept data

Intercept of radiocarbon age with calibration curve:

1 Sigma calibrated result: Cal BC 8595 to 8320 (Cal BP 10545 to 10270 ) ( $68 \%$ probability)

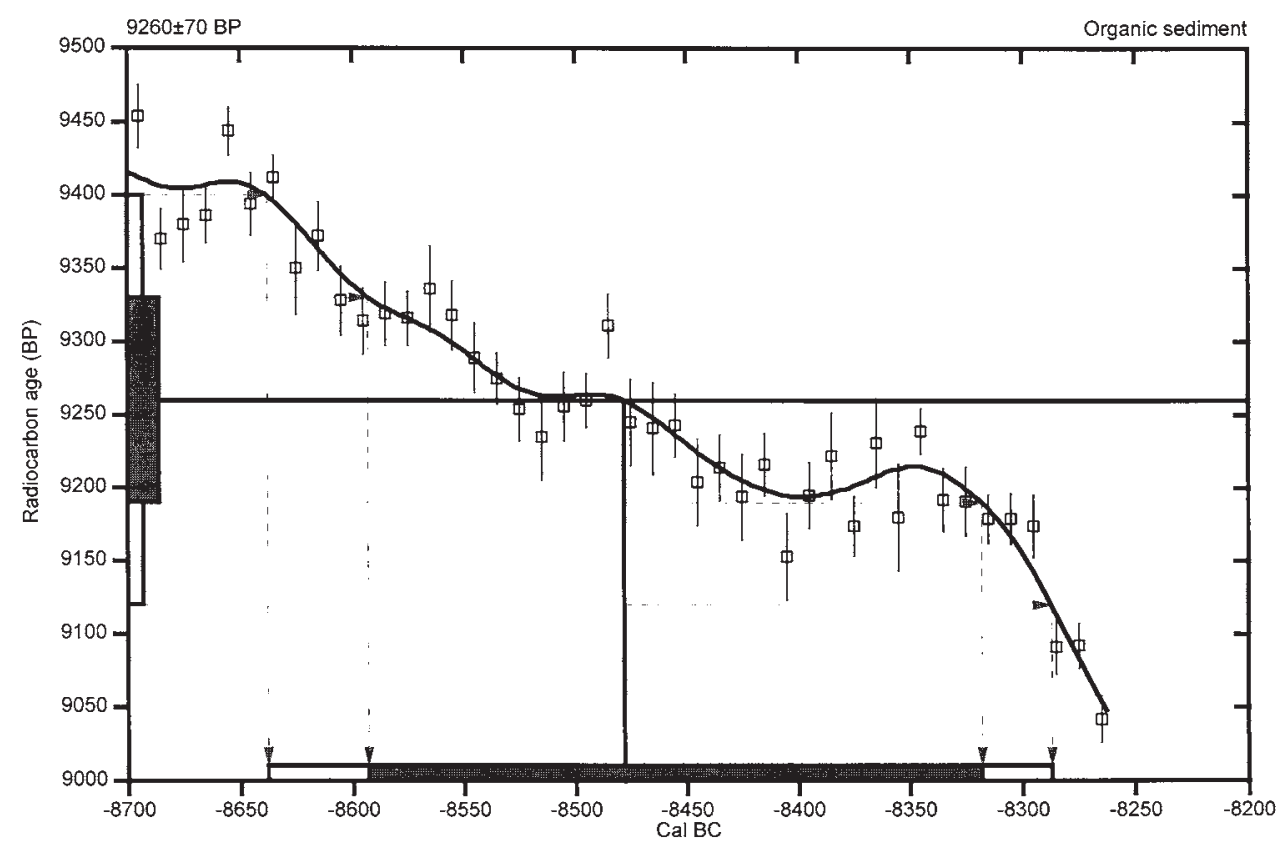

References:

Database used

CA98

Calibration Databas

Editorial Comment

Stuiver, M., van der Plicht, H., I998, Radiocarbon 40(3), pxii-xiii

INTCAL98 Radiocarbon Age Calibration

Stuiver, M., et. al., 1998, Radiocarbon 40(3), p1041-1083

Mathematics

A Simplified Approach to Calibrating C14 Dates

Talma, A. S., Vogel, J. C., 1993, Radiocarbon 35(2), p317-322

\section{Beta Analytic Radiocarbon Dating Laboratory}

4985 S.W. 74th Court, Miami, Florida 33155 - Tel: (305)667-5167 • Fax: (305)663-0964 • E-mail: beta@radiocarbon.com 


\section{CALIBRATION OF RADIOCARBON AGE TO CALENDAR YEARS}

(Variables: C13/C12=-15:lab. mult=1)

Laboratory number: Beta-136276

Conventional radiocarbon age: $3360 \pm 50 \mathrm{BP}$

2 Sigma calibrated result: Cal BC 1750 to 1520 (Cal BP 3700 to 3470)

(95\% probability)

Intercept data

Intercept of radiocarbon age

with calibration curve: Cal BC 1650 (Cal BP 3600)

1 Sigma calibrated result: Cal BC 1700 to 1600 (Cal BP 3650 to 3550 )

(68\% probability)

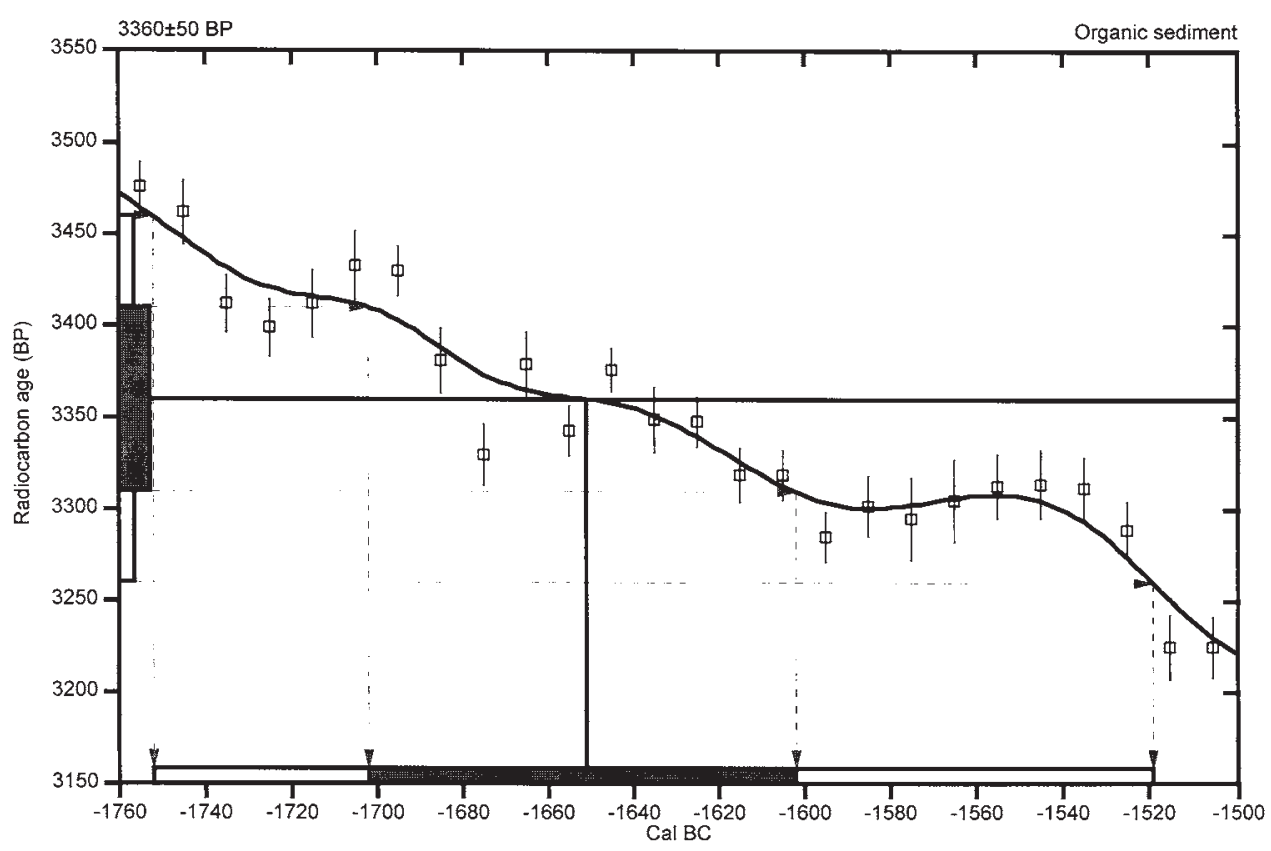

References:

Database used

INTCAL98

Calibration Database

Editorial Comment

Editorial Comment
Stuiver, $M$, van der Plicht, H., 1998, Radiocarbon 40(3), pxii-xili
INTCAL 98 Radiocarbon Age Calibration

Stuiver, M., et. al., 1998, Radiocarbon 40(3), p1041-1083

Mathematics

A Simplified Approach to Calibrating C14 Dates

Talma, A. S., Vogel, J. C., 1993, Radiocarbon 35(2), p317-322

\section{Beta Analytic Radiocarbon Dating Laboratory}

4985 S.W. 74th Court, Miami, Florida 33155 • Tel: (305)667-5167 • Fax: (305)663-0964 • E-mail: beta@radiocarbon. com 


\section{CALIBRATION OF RADIOCARBON AGE TO CALENDAR YEARS}

(Variables: $\mathrm{C} 13 / \mathrm{C} 12=-17.5$ :lab. mult $=1$ )

Laboratory number: Beta-136277

Conventional radiocarbon age: $910 \pm 60 \mathrm{BP}$

2 Sigma calibrated result: Cal AD 1005 to 1255 (Cal BP 945 to 695)

(95\% probability)

Intercept data

Intercept of radiocarbon age

with calibration curve:

1 Sigma calibrated result: Cal AD 1030 to 1205 (Cal BP 920 to 745)

( $68 \%$ probability)

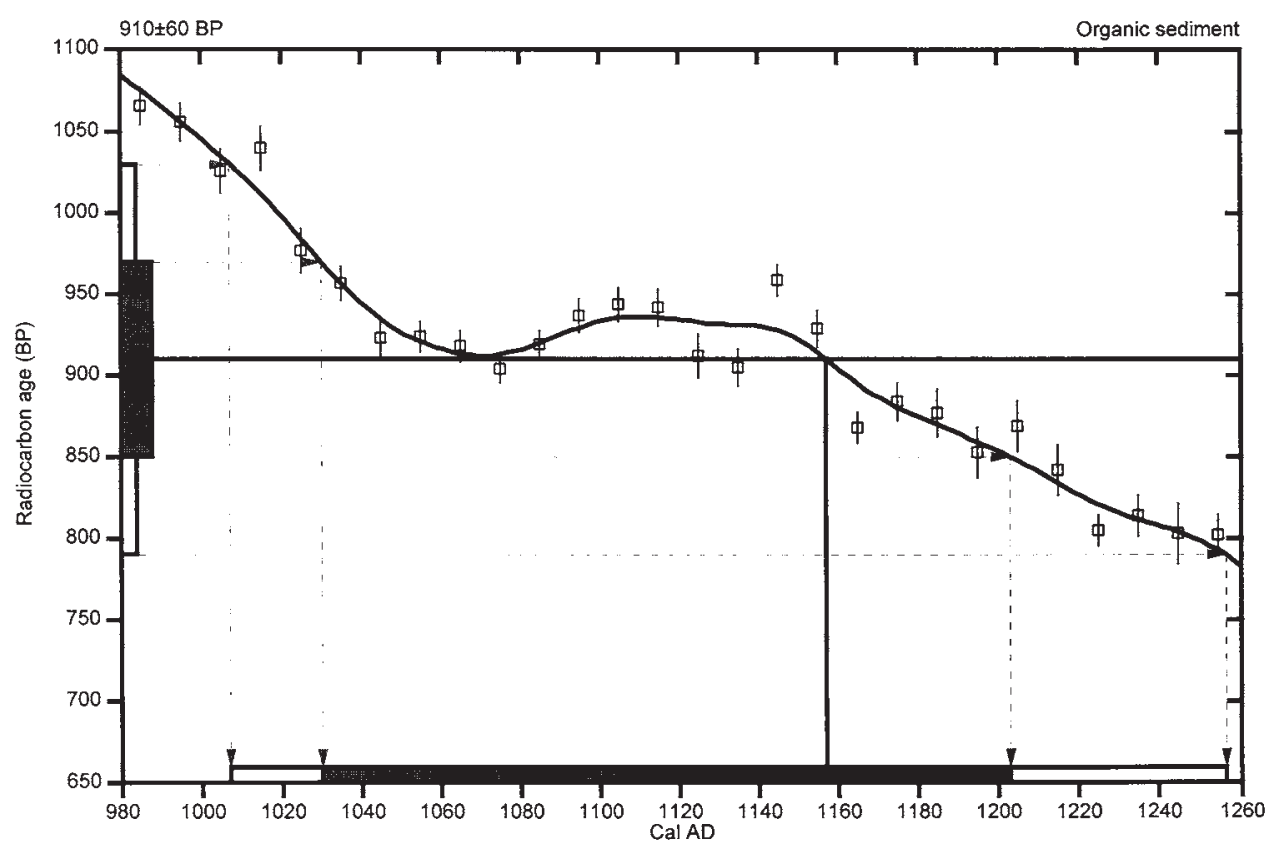

References:

Database used

INTCAL98

Calibration Database

Editorial Comment

Editorial Comment
Stuiver, M., van der Plicht, H. 1998, Radiocarbon 40(3), pxii-xiii

Stuiver, M, van der Plicht, $H ., 1998$,
INTCAL98 Radiocarbon Age Calibration

Stuiver, M., et. al., I998, Radiocarbon 40(3), pI04I-1083

Mathematics

A Simplified Approach to Calibrating C14 Dates

Talma, A. S., Vogel, J. C., 1993, Radiocarbon 35(2), p317-322

\section{Beta Analytic Radiocarbon Dating Laboratory}

4985 S.W. 74th Court, Miami, Florida 33155 • Tel: (305)667-5167 • Fax: (305)663-0964 • E-mail: beta@radiocarbon.com 


\section{CALIBRATION OF RADIOCARBON AGE TO CALENDAR YEARS}

(Variables: $\mathrm{C} 13 / \mathrm{C} 12=-23.6:$ lab. mult $=1$ )

Laboratory number: Beta-136278

Conventional radiocarbon age: $5760 \pm 70 \mathrm{BP}$

2 Sigma calibrated result: Cal BC 4765 to 4455 (Cal BP 6715 to 6405)

(95\% probability)

Intercept data

Intercept of radiocarbon age

with calibration curve: Cal BC 4595 (Cal BP 6545)

1 Sigma calibrated result: Cal BC 4705 to 4515 (Cal BP 6655 to 6465 )

(68\% probability)

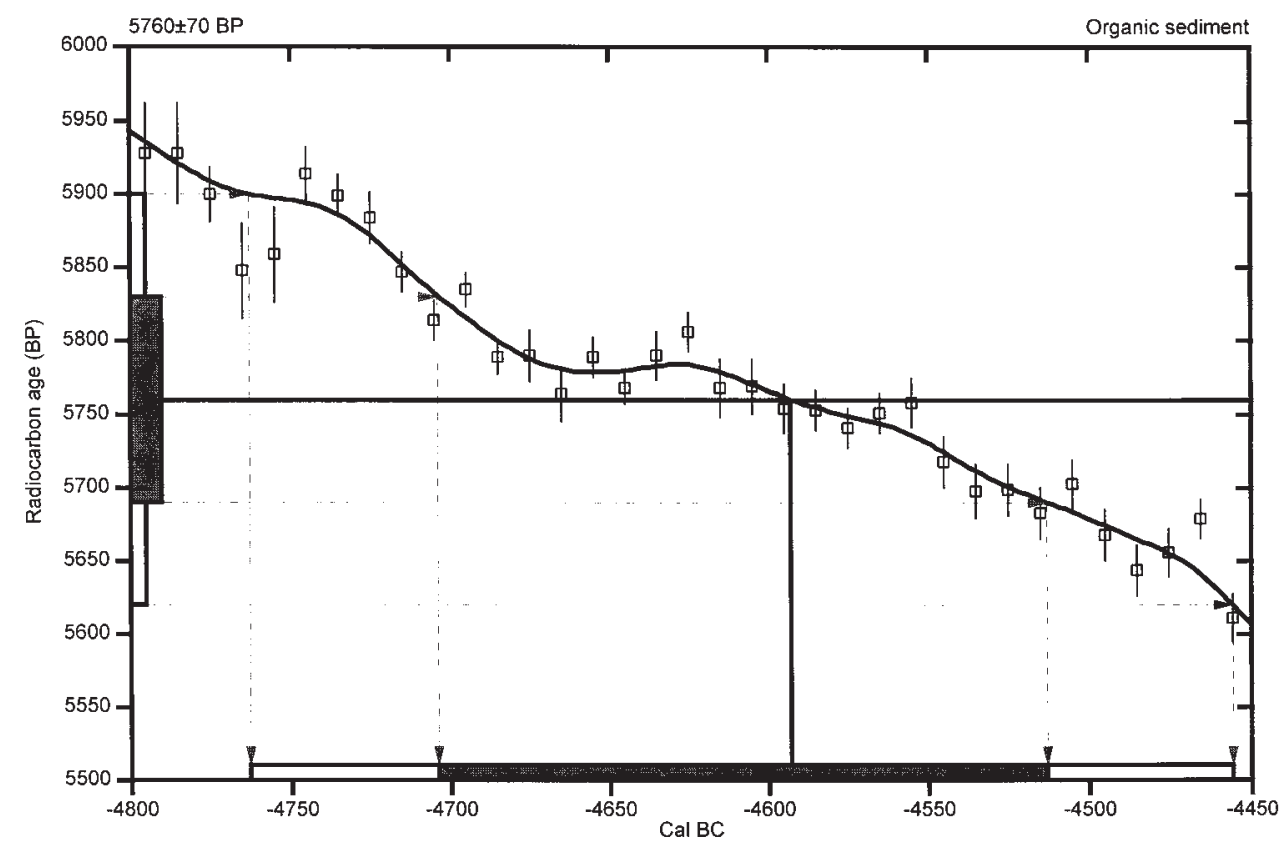

References:
Database used
INTCAL98
Calibration Database
Editorial Comment
Stuiver, M., van der Plicht, H., 1998. Radiocarbon 40(3), pxii-xii
INTCAL98 Radiocarbon Age Callbration
Stuiver, M., et. al., 1998, Radiocarbon 40(3), pl041-1083
Mathematics
A Simplified Approach to Calibrating C14 Dates
Talma, A. S., Vogel, J. C., 1993, Radiocarbon 35(2), p317-322

\section{Beta Analytic Radiocarbon Dating Laboratory}

4985 S.W.74th Court, Miami, Florida 33155 - Tel: (305)667-5167• Fax: (305)663-0964 • E-mail: beta@radiocarbon.com 


\section{CALIBRATION OF RADIOCARBON AGE TO CALENDAR YEARS}

(Variables: $\mathrm{C} 13 / \mathrm{C} 12=-18.8$ :lab. mult $=1$ )

Laboratory number: Beta-136279

Conventional radiocarbon age: $3460 \pm 60 \mathrm{BP}$

2 Sigma calibrated result: Cal BC 1920 to 1625 (Cal BP 3870 to 3575) (95\% probability)

Intercept data

Intercept of radiocarbon age with calibration curve:

1 Sigma calibrated result: Cal BC 1880 to 1695 (Cal BP 3830 to 3645) (68\% probability)

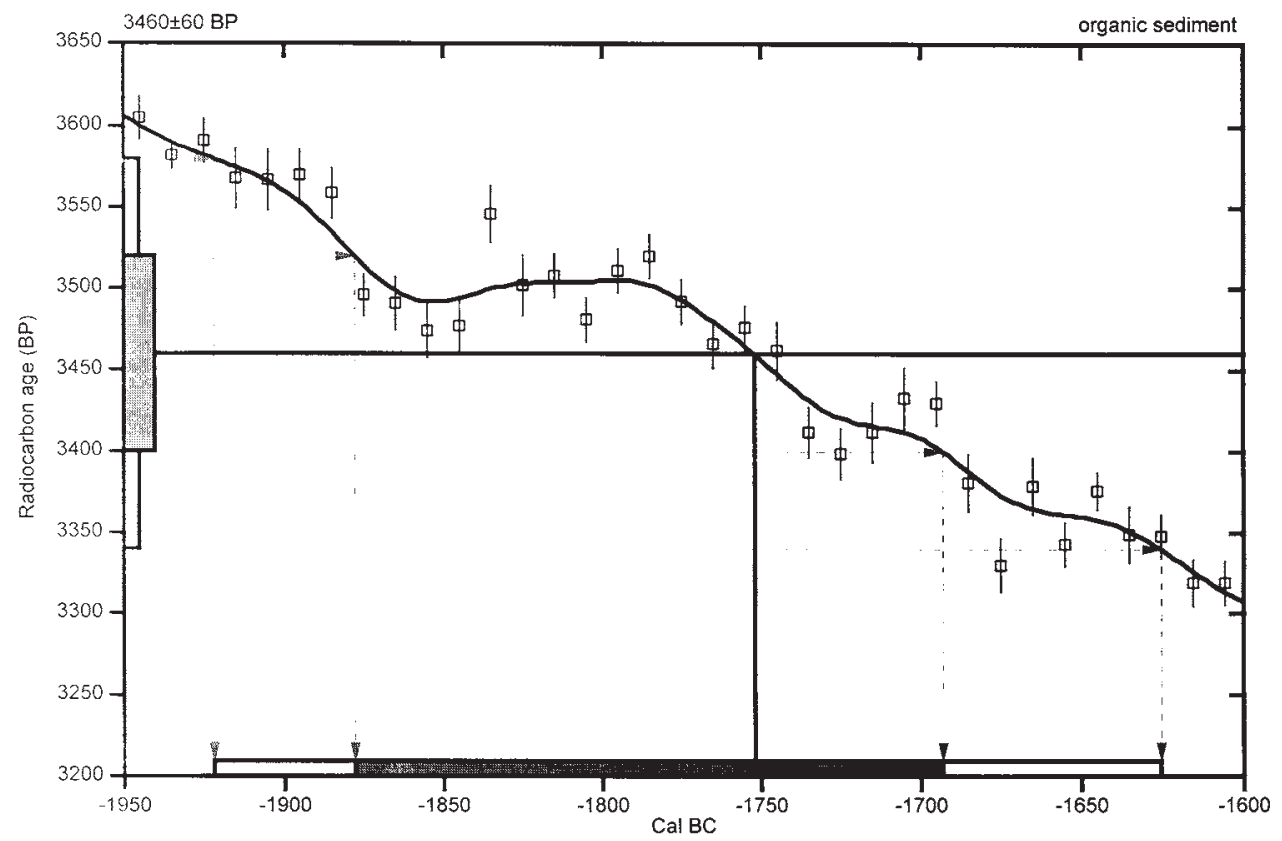

References:

Database used

Calibration Database

Editorial Comment

Stuiver, M., van der Plicht, H., 1998, Radiocarbon 40(3), pxii-xiit

INTCAL98 Radiocarbon Age Calibration

Stuiver, M., et. al. 1998, Radiocarbon 4013), p1041-1083

Mathematics

A Simplified Approach to Calibrating C14 Dates

Talma, A. S., Vogel, J. C., 1993, Radiocarbon 35(2), p317-322

\section{Beta Analytic Radiocarbon Dating Laboratory}

1955.5.11: 74th Court, Miami, Florida 33155 • Tel: (305)667-5167 • Fax: (305)663-0964 • E-mail: beta@radiocarbon.com 


\section{CALIBRATION OF RADIOCARBON AGE TO CALENDAR YEARS}

(Variables: $\mathrm{C} 13 / \mathrm{C} 12=-18.4$ :lab. mult=1)

Laboratory number: Beta-136280

Conventional radiocarbon age: $3440 \pm 50 \mathrm{BP}$

2 Sigma calibrated result: Cal BC 1890 to 1625 (Cal BP 3840 to 3575)

(95\% probability)

Intercept data

Intercept of radiocarbon age

with calibration curve: Cal BC 1740 (Cal BP 3690)

1 Sigma calibrated result: Cal BC 1770 to 1685 (Cal BP 3720 to 3635) ( $68 \%$ probability)

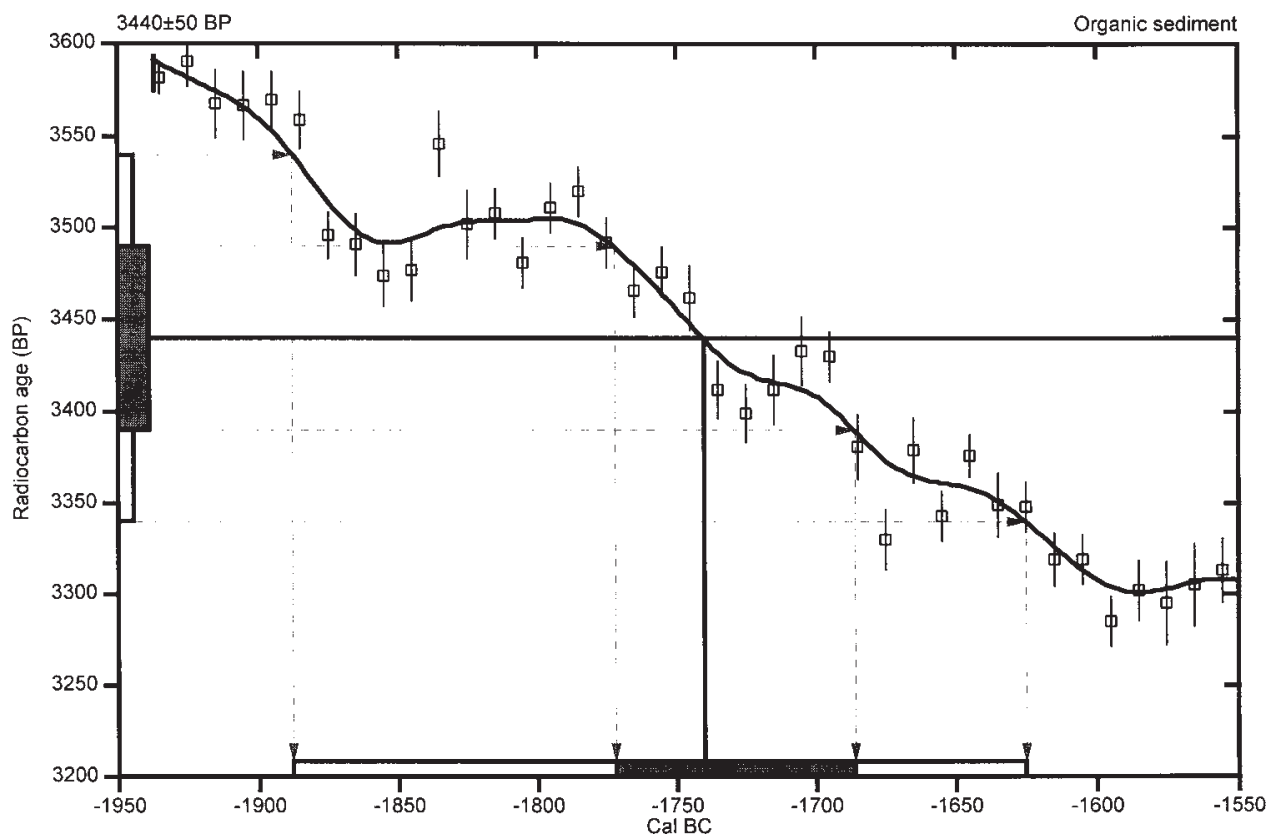

References:

Database used

INTCAL98

Calibration Database

Editorial Comment

Stuiver, M., van der Plicht, H., 1998, Radiocarbon 40(3), pxii-xiit

INTCAL98 Radiocarbon Age Calibration

Stuiver, M., et. al., 1998, Radiocarbon 40(3), pl041-1083

Mathematics

A Simplified Approach to Calibrating C14 Dates

Talma, A. S., Vogel, J. C., 1993, Radiocarbon 35(2), p317-322

Beta Analytic Radiocarbon Dating Laboratory

4985 S.W. 74th Court, Miami, Florida 33155 •Tel: (305)667-5167 • Fax: (305)663-0964•E-mail: beta@radiocarbon.com 


\section{BETA ANALYTIC INC.}

DR. M.A. TAMERS and MR. D.G. HOOD
UNIVERSITY BRANCH

4985 S.W. 74 COURT

MIAMI, FLORIDA, USA 33155

PH: 305/667-5167 FAX: 305/663-0964

E-MAIL: beta@radiocarbon.com

\section{REPORT OF RADIOCARBON DATING ANALYSES}

Dr. Raymond Mauldin

University of Texas at San Antonio
Report Date: January 19, 2000

Material Received: Auth. Dec. 23, 1999

\begin{tabular}{lccc}
\hline Sample Data & $\begin{array}{c}\text { Measured } \\
\text { Radiocarbon Age }\end{array}$ & $\begin{array}{c}{ }^{13} \mathrm{C} /{ }^{12} \mathrm{C} \\
\text { Ratio }\end{array}$ & $\begin{array}{c}\text { Conventional } \\
\text { Radiocarbon Age }(*)\end{array}$ \\
\hline Beta-136275 & $7240+/-40 \mathrm{BP}$ & $-19.0 \mathrm{o} / 00$ & $7340+1-40 \mathrm{BP}$
\end{tabular}

SAMPLE \#: BHT4Ab2

ANALYSIS: Standard-AMS

MATERIAL/PRETREATMENT:(organic sediment): acid washes

NOTE: It is important to read the calendar calibration information and to use the calendar calibrated results (reported separately) when interpreting these results in $\mathrm{AD} / \mathrm{BC}$ terms.

Dates are reported as RCYBP (radiocarbon years before present, "present" = 1950A.D.). By International convention, the modern reference standard was $95 \%$ of the C14 content of the National Bureau of Standards' Oxalic Acid \& calculated using the Libby C14 half life (5568 years). Quoted errors represent 1 standard deviation statistics ( $68 \%$ probability) \& are based on combined measurements of the sample, background, and modern reference standards.
Measured $\mathrm{C} 13 / \mathrm{C} 12$ ratios were calculated relative to the PDB-1 international standard and the RCYBP ages were normalized to -25 per mil. If the ratio and age are accompanied by an $\left({ }^{*}\right)$, then the $\mathrm{C} 13 / \mathrm{C} 12$ value was estimated, based on values typical of the material type. The quoted results are NOT calibrated to calendar years. Calibration to calendar years should be calculated using the Conventional $\mathrm{C} 14$ age. 


\section{CALIBRATION OF RADIOCARBON AGE TO CALENDAR YEARS}

(Variables: C13/C12=-19:1ab. mult=1)

Laboratory number: Beta-136275

Conventional radiocarbon age: $\quad \mathbf{7 3 4 0} \pm 40 \mathrm{BP}$

2 Sigma calibrated result: Cal BC 6245 to 6080 (Cal BP 8195 to 8030) (95\% probability)

Intercept data

Intercept of radiocarbon age

with calibration curve: Cal BC 6215 (Cal BP 8165)

1 Sigma calibrated result: Cal BC 6230 to 6185 (Cal BP 8180 to 8135) ( $68 \%$ probability)

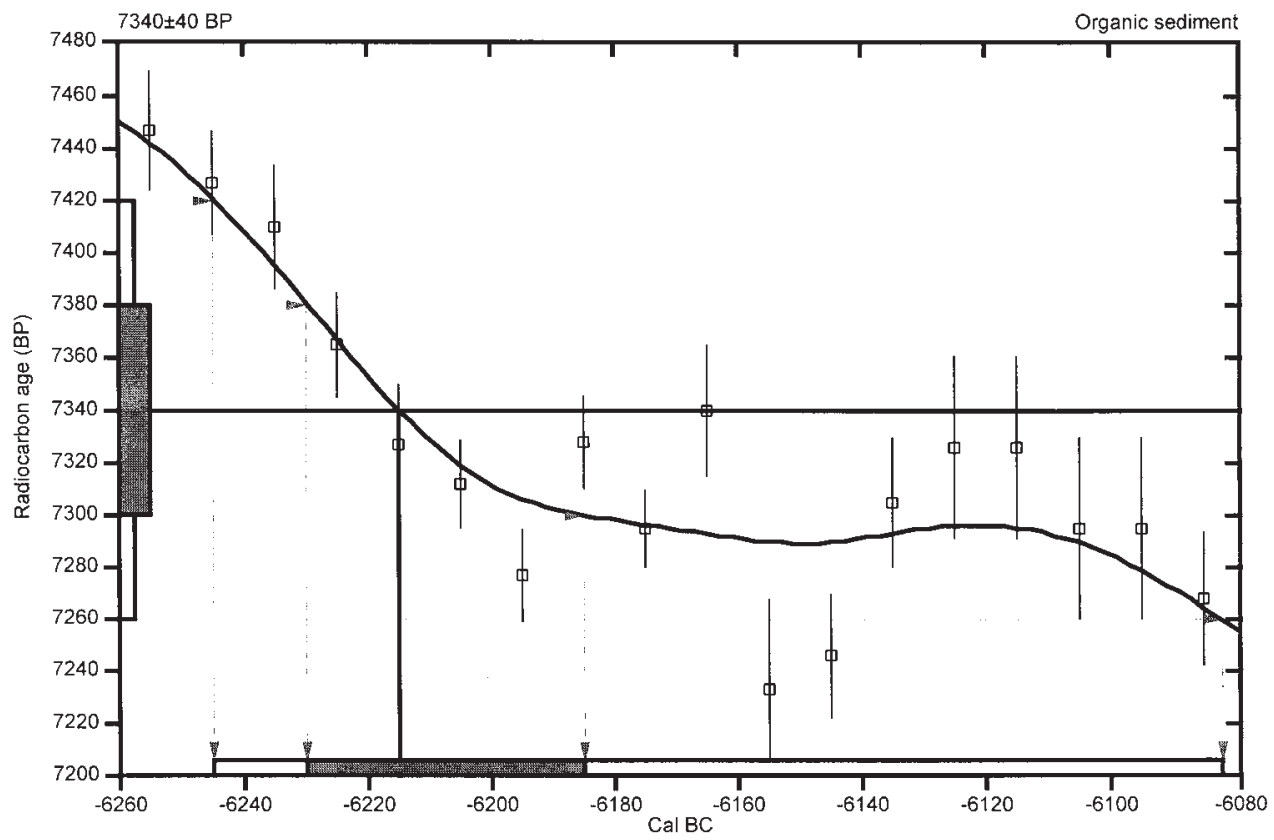

References:

Database used
INTCAL98

Calibration Database

Editorial Comment

Stuiver, M., van der Plicht, H., 1998, Radiocarbon 40(3), pxii-xiii

INTCAL 98 Radiocarbon Age Calibration

Stuiver, M., et. al., 1998, Radiocarbon 40(3), p1041-1083

Mathematics

A Simplified Approach to Calibrating C14 Dates

Talma, A. S., Vogel, J. C., 1993, Radiocarbon 35(2), p317-322

Beta Analytic Radiocarbon Dating Laboratory

4985 S.W. 74th Court, Mami, Florida 33155 - Tel: (305)667-5167 • Fax: (305)663-0964 - E-mail: heta@radiocarbon.com 


\section{Appendix I: \\ Twin Buttes Archaeological Project}

\section{Soil-Stratigraphic Descriptions}

\section{Middle Concho River}

CB1a; Modern alluvium (MO) over Late Holocene alluvium (LH); Holocene flood terrace (6 m); calcareous throughout.

A/C1 0-18 cm; very dark grayish brown (10YR 3/2) silt loam; weak coarse subangular blocky; hard; common fine worm casts; clear smooth.

A/C1 18-43 cm; very dark grayish brown (10YR 3.5/2) silt loam; weak coarse subangular blocky; hard; few laminated clasts; common fine worm casts; clear smooth. Hearth charcoal radiocarbon age of $870 \pm 40$ B.P. in lower part.

$\mathrm{Ab} \quad$ 43-67 cm; very dark grayish brown (10YR 3/2) silt loam; moderate medium prismatic; hard; $1 \%$ carbonate filaments; common fine biocasts; gradual smooth.

Bw1b 67-100 cm; very dark grayish brown (10YR 3.5/2) silt loam; moderate coarse prismatic; hard; $1 \%$ carbonate filaments; few fine biocasts; hearth at upper contact; gradual smooth.

Bw2b 100-151 cm; brown (10YR 4/3) silt loam; weak coarse prismatic; hard; $1 \%$ carbonate filaments; few fine biocasts; gradual smooth.

Bw3b 221- $268 \mathrm{~cm}$; brown (10YR 4/3) silt loam; weak coarse prismatic; hard; 1\% carbonate filaments; few fine biocasts; gradual smooth.

Bw4b 268-322 cm; brown (10YR 4/3) silt loam; weak coarse prismatic; hard; few fine biocasts; dispersed charcoal sample; gradual smooth.

Bw5b 322-348 cm; brown (10YR 4/3, 5/3) silt loam; weak coarse prismatic; hard; few fine biocasts; clear smooth.

Bw6b 348-364 cm; brown (10YR 3.5/3) silt loam; weak coarse prismatic; slightly hard; few fine biocasts; gradual smooth.

Bw1b 364-418 cm; brown (10YR 3.5/3) silty clay loam; 10\% brown (10YR 5/3); weak coarse prismatic; slightly hard; few fine biocasts; clear smooth.

Bw2b 418-452 cm; brown (10YR 3.5/3) silty clay loam; 10\% brown (10YR 5/3); weak coarse prismatic; slightly hard; few fine biocasts; few fine clay clasts; clear smooth. 
Bw3b 452- $483 \mathrm{~cm}$; brown (10YR 4/3) silty clay loam; 20\% brown (10YR 5/3); weak coarse prismatic; slightly hard; few fine biocasts; few fine clay clasts; gradual smooth.

Bkb 483-520 cm; brown (10YR 4/3) silty clay loam; 15\% brown (10YR 5/3); weak coarse prismatic; very hard; $1 \%$ carbonate nodules, 0.5 to $1.5 \mathrm{~cm}$, white, very hard; few fine biocasts; few fine clay clasts; gradual smooth.

BCkb 520-593 cm; brown (10YR 4/3, 5/3) and pale brown (10YR 6/3) silty clay loam; 15\% brown (10YR 5/ 3 ); weak coarse prismatic; very hard; common faint laminations; $2 \%$ carbonate nodules, 0.5 to $1.5 \mathrm{~cm}$, white, very hard; $1 \%$ carbonate filaments; few fine biocasts; few fine clay clasts; gradual smooth.

$\mathrm{BCb} 593-634 \mathrm{~cm}$; bedded dark grayish brown (10YR 3.5/2) and brown (10YR 4/3) clay loam, and pale brown (10YR 6/3) silt loam, 2 to $10 \mathrm{~cm}$ thick; common faint laminations; massive; hard; $1 \%$ carbonate filaments.

CB1b; Modern alluvium (MO); modern floodplain (4 m); calcareous throughout.

A/C1 0-17 cm; dark brown (10YR 3/3) sandy clay loam; moderate fine subangular blocky; friable; common fine biocasts; common uncoated sand particles; gradual smooth.

A/C2 17-35 cm; very dark grayish brown (10YR 3/2) sandy clay loam; moderate medium subangular blocky; friable; common fine biocasts; common uncoated sand particles; gradual smooth.

Ab1 35-66 cm; very dark gray (10YR 3/1) loam; moderate medium subangular blocky; friable; gradual smooth.

Cb1 66-89 cm; very dark grayish brown (10YR 3/2) clay loam with $3(0.5 \mathrm{~cm})$ yellowish brown (10YR 5/4) beds; massive to weak coarse subangular blocky; slightly hard; clear smooth.

Ab2 89-119 cm; very dark gray (10YR 3/1) clay loam; 10\% dark grayish brown (10YR 4/2); weak coarse subangular blocky; hard; gradual smooth.

C1b2 119-146 cm; dark grayish brown (10YR 4/2) sandy clay loam; 20\% pale brown (10YR 6/3); massive; hard; faint bedding planes; gravel line in middle and bottom, 0.5 to $1 \mathrm{~cm}$, subrounded, grain supported; abrupt smooth.

C2b2 146-212 cm; multiple beds 3 to $10 \mathrm{~cm}$ of very dark grayish brown (10YR 3/2) clay loam and pale brown loam (10YR 6/3) with $40 \%$ pebbles, 0.2 to $0.5 \mathrm{~cm}$, subrounded; abrupt smooth. Bulk humate radiocarbon age of $910 \pm 60$ B.P. in lower part.

C1b2 212-247 cm; 80\% pebbles, 0.4 to $1 \mathrm{~cm}$ in upper half and 1 to $4 \mathrm{~cm}$ in lower half, moderately well sorted, subrounded, grain supported; abrupt smooth.

C2b2 247-394 cm; multiple light tan cross beds 10 to $50 \mathrm{~cm}$ thick, 50 to $80 \%$ pebbles, mostly subrounded, moderately sorted, 0.2 to $1 \mathrm{~cm}$ and 2 to $7 \mathrm{~cm}$ diameter.

Cb2 394-425 cm; dark brown (10YR 3/3); upper $10 \mathrm{~cm}$ massive clay loam with few fine pebbles, lower part $60 \%$ pebbles, 0.5 to $4 \mathrm{~cm}$, mostly subrounded, moderately sorted, matrix supported. 
CB2a; Latest Pleistocene alluvium (LP2); latest Pleistocene terrace (8-9 m); calcareous throughout; approximately upper $1 \mathrm{~m}$ truncated.

Bk 0-34 cm; brown (10YR 5.5/3) clay loam; moderate medium subangular blocky; hard; 5\% carbonate nodules, 0.5 to $1.5 \mathrm{~cm}$, white, slightly hard; $20 \%$ pebbles, 0.3 to $2 \mathrm{~cm}$, subrounded, matrix supported, moderately sorted; gradual smooth.

BC $\quad 34-54 \mathrm{~cm}$; brown (7.5YR 5/4) clay loam; 30\% light brownish gray (10YR 6/2); moderate coarse angular blocky; hard; common disseminated carbonate; clear smooth.

BC 54-85 cm; brown (7.5YR 5.5/4) clay loam; moderate medium angular blocky; hard; 3\% carbonate nodules, $0.5 \mathrm{~cm}$, white, slightly hard; clear smooth.

Bkg $\quad 85-133 \mathrm{~cm}$; light brownish gray (2.5Y 6/2) clay; moderate medium angular blocky; hard; $30 \%$ carbonate nodules, 0.5 to $2 \mathrm{~cm}$, white, slightly hard to hard; gradual smooth.

BCg1 133-151 cm; light gray (2.5Y 7/2) clay loam; weak coarse angular blocky; firm; $1 \%$ pebbles, $1 \mathrm{~cm}$, subrounded; clear smooth.

BCg2 151-191 cm; light gray (2.5Y 7.5/2) sandy clay loam; weak coarse angular blocky; firm; common medium distinct light olive brown (2.5Y 5/6) soft iron masses; clear smooth.

$\mathrm{Cg} \quad 191-201 \mathrm{~cm}$; light gray (2.5Y 7.5/2) clay loam; massive; very firm; $1 \%$ carbonate nodules, 0.2 to $0.5 \mathrm{~cm}$, white, slightly hard; abrupt wavy. Bulk humate radiocarbon sample of $5760 \pm 70$ в.P.

C1 201-301 cm; 70\% pebbles, 1 to $8 \mathrm{~cm}$, subrounded, grain supported, moderate sorted; tan loamy matrix with few iron stains; clear wavy.

C2 $\quad 301-581 \mathrm{~cm} ; 70 \%$ pebbles, multiple beds and cross beds, 1 to $8 \mathrm{~cm}$ and 0.2 to $0.5 \mathrm{~cm}$, subrounded, grain supported, moderate sorted; occasional bedrock rip up clasts and cobbles up to $15 \mathrm{~cm}$ in lower half.

CB2b; Latest Pleistocene alluvium (LP2); latest Pleistocene terrace (8-9 m); calcareous throughout; approximately upper $1 \mathrm{~m}$ truncated.

BA 0-19 cm; brown (10YR 5/4) clay loam; moderate medium prismatic to moderate medium subangular blocky; very hard; $2 \%$ pebbles, 0.2 to $0.4 \mathrm{~cm}$, subrounded to angular, matrix supported, moderately well sorted; gradual smooth.

Bk1 19 -39 cm; brown (10YR 5/4) clay loam; moderate medium prismatic to moderate medium angular blocky; very hard; $2 \%$ pebbles, 0.2 to $0.4 \mathrm{~cm}$, subrounded to angular, matrix supported, moderately well sorted; $2 \%$ carbonate nodules, $1 \mathrm{~cm}$, white, slightly hard to hard; gradual smooth.

Bk2 39-51 cm; brown (10YR 5/4) clay loam; moderate medium angular blocky; very hard; 5\% carbonate nodules, $1 \mathrm{~cm}$, white, slightly hard to hard; $3 \%$ carbonate filaments; clear smooth.

B/Ck 51-70 cm; reddish brown (5YR 5/4) clay loam; common medium distinct yellowish brown (10YR 5/6) soft iron masses; faintly bedded; firm; $3 \%$ carbonate nodules, $1 \mathrm{~cm}$, white, slightly hard to hard; $3 \%$ carbonate filaments; abrupt smooth. 
Ck 70-98 cm; reddish brown (5YR 5/4) loam; common medium distinct yellowish brown (10YR 5/6) soft iron masses; massive; firm; $1 \%$ carbonate nodules, $0.5 \mathrm{~cm}$, white, slightly hard to hard; $3 \%$ carbonate filaments; abrupt smooth.

C1 98-200 $\mathrm{cm} ; 60 \%$ pebbles, 0.2 to $2 \mathrm{~cm}$, subrounded, moderate sorted, grain supported and dipping; tan loamy matrix; abrupt wavy.

C2 200-500 cm; 70\% pebbles, 1 to $15 \mathrm{~cm}$, subrounded, moderate sorted, grain supported; tan loamy alluvium.

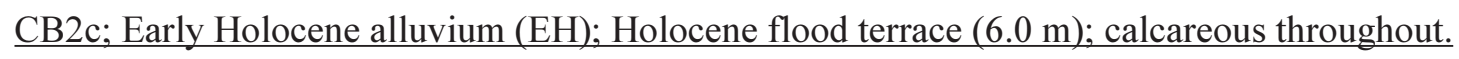

C 0-3 cm; very dark gray (10YR 3/1) loam; moderate medium platy; hard; abrupt wavy. (lake deposit)

A1 3-24 cm; very dark gray (10YR 3/2.5) clay loam; few fine distinct brown (7.5YR 4/4) iron pore linings; weak medium angular blocky; very hard; gradual smooth.

A2 24-53 cm; very dark grayish brown (10YR 3/2) clay loam; weak medium prismatic; very hard; gradual smooth.

Bw $\quad 53-91 \mathrm{~cm}$; brown (10YR 3.5/3) clay loam; weak medium prismatic; very hard; gradual smooth.

Bk1 91-119 cm; brown (7.5YR 4/4) clay loam; weak medium prismatic to weak medium angular blocky; very hard; $1 \%$ carbonate filaments; $5 \%$ pebbles, 0.2 to $0.4 \mathrm{~cm}$, subrounded, matrix supported, moderately well sorted; gradual smooth.

Bk2 119-185 cm; brown (7.5YR 4/4.5) clay loam; weak medium prismatic to weak medium angular blocky; very hard; $1 \%$ carbonate filaments; $1 \%$ pebbles, 0.5 to $1 \mathrm{~cm}$, subrounded, matrix supported and $5 \%$ pebbles, 0.2 to $0.4 \mathrm{~cm}$, subrounded, matrix supported, moderately well sorted; clear smooth.

Bk3 185-239 cm; brown (7.5YR 4/3) clay loam; weak coarse subangular blocky; firm; 2\% carbonate filaments; $5 \%$ pebbles, 0.3 to $1 \mathrm{~cm}$; subrounded, matrix supported, moderately well sorted; clear wavy.

C 239-275 cm; light brown (7.5YR 6/4) loam; 40\% pebbles, 0.5 to $3 \mathrm{~cm}$, subrounded, grain supported.

CB3 Early Holocene alluvium (EH); truncated Holocene flood terrace (6 m); calcareous throughout.

Bk1 $\quad 0-80 \mathrm{~cm} ; 70 \%$ pebbles, 0.2 to $0.6 \mathrm{~cm}$ and 1 to $6 \mathrm{~cm}$, moderately sorted; grain supported, subrounded; 1 to $3 \mathrm{~mm}$ thick discontinuous carbonate pendants; brown (10YR 5/3) clay loam matrix; abrupt wavy.

Bk2 80-112 cm; brown (7.5YR 4/4) clay loam; 20\% brown (7.5YR 5/3); weak coarse prismatic; very firm; $3 \%$ carbonate nodules, 0.5 to $1.5 \mathrm{~cm}$, white, soft to hard; few biocasts.

Bw1 112-130 cm; brown (7.5YR 4/3) and light brown (7.5YR 6/3) clay loam; weak coarse prismatic; very firm; gradual smooth.

Bw2 130-160 cm; brown (7.5YR 4/3, 5/4) clay loam; weak coarse prismatic; firm; common fine distinct iron manganese root pores; gradual smooth. 
BC 160-220 cm; pale brown (10YR 6/3) clay loam; $3 \%$ pebbles, 0.5 to $1 \mathrm{~cm}$, subrounded, matrix supported; clear smooth.

C1 220-235 cm; light brown (7.5YR 6/4) clay loam; 15\% pebbles, 1 to $3 \mathrm{~cm}$, matrix supported, moderately sorted, subrounded; clear smooth.

C2 235-285 cm; 70\% pebbles, 0.2 to $0.5 \mathrm{~cm}$ and 1 to $5 \mathrm{~cm}$, moderately to poorly sorted; subrounded, grain supported; tan matrix; abrupt wavy.

C3 285-400 cm; very pale brown (10YR 7/3) sandy clay loam; few medium faint yellow iron soft masses; three pebbles beds, $10 \mathrm{~cm}$ thick, 1 to $3 \mathrm{~cm}$, subrounded, grain supported, moderately well sorted; abrupt wavy.

C4 $400-440 \mathrm{~cm} ; 80 \%$ pebbles, 1 to $8 \mathrm{~cm}$, grain supported, moderately sorted, subrounded; loamy gray matrix.

BHT-1; Modern alluvium (MO) over Early Holocene alluvium (EH); Holocene flood terrace (6.5 m); calcareous throughout.

A $\quad 0-9 \mathrm{~cm}$; dark grayish brown (10YR 3.5/2) silt loam; 10\% brown (10YR 4/3); moderate fine and medium platy; slightly hard; many biocasts; clear smooth.

$\mathrm{Ab} \quad 9-51 \mathrm{~cm}$; very dark grayish brown (10YR 3/2) silty clay loam; moderate medium and coarse angular blocky; hard; many biocasts; gradual smooth.

Bw1b 51-80 cm; dark yellowish brown (10YR 4/3.5) silty clay loam; moderate medium prismatic to moderate medium angular blocky; hard; common biocasts; gradual smooth.

Bw2b 80-112 cm; dark yellowish brown (10YR 4/4) silty clay loam; weak coarse prismatic; hard; common biocasts; few pebbles, subrounded, $1 \mathrm{~cm}$; gradual smooth.

Bw3b 112-147 cm; dark yellowish brown (10YR 4/4) silty clay loam; weak coarse prismatic; very hard; few biocasts; few pebbles, subrounded, $1 \mathrm{~cm}$; clear smooth.

Bkb 147-209 cm; dark yellowish brown (10YR 4/4) silty clay loam; weak coarse prismatic to weak coarse angular blocky; very hard; few biocasts; $2 \%$ carbonate filaments; gradual smooth.

BCb 209-279 cm; brown (10YR 4/3) and yellowish brown (10YR 5/4) silty clay loam; 5\% yellowish brown (7.5YR 5/4) sandy patches; weak coarse prismatic; very hard; few biocasts; few pebbles, subrounded, $1 \mathrm{~cm}$.

BHT-2; late Pleistocene alluvium (LP1); late Pleistocene terrace (12-14 m); calcareous throughout.

A $\quad 0-16 \mathrm{~cm}$; very dark grayish brown (10YR 3/2) clay loam; moderate medium subangular blocky; hard; $3 \%$ pebbles, 0.5 to $1 \mathrm{~cm}$, angular to subrounded, matrix supported, moderately sorted; gradual smooth. 
BA 16-35 cm; yellowish brown (10YR 5/4) clay loam; 30\% very dark grayish brown (10YR 3/2) biocasts; moderate medium to coarse angular blocky; hard; $4 \%$ carbonate clasts, 0.5 to $1 \mathrm{~cm}$, white, slightly hard; $3 \%$ pebbles, 0.2 to $0.4 \mathrm{~mm}$, angular to subrounded, moderate sorted, matrix supported; gradual smooth.

Bw $\quad 35-53 \mathrm{~cm}$; brown (7.5YR 5/4) clay loam; few fine faint strong brown (7.5YR 4/6) soft iron masses; $2 \%$ very dark grayish brown (10YR 3/2) biocasts; moderate coarse angular blocky; very hard; $1 \%$ carbonate clasts, 0.5 to $1 \mathrm{~cm}$, white, slightly hard; $1 \%$ pebbles, 0.2 to $0.4 \mathrm{~cm}$, angular to subrounded, moderate sorted, matrix supported; clear smooth.

Bk1b 53-74 cm; brown (7.5YR 5/4) clay; moderate medium angular blocky; very hard; $15 \%$ carbonate nodules, 0.5 to $1.5 \mathrm{~cm}$, white, slightly hard to hard; clear smooth.

Bk2b 74-144 cm; yellowish brown (5YR 5/4) clay; moderate medium prismatic to moderate medium angular blocky; very hard; $20 \%$ carbonate nodules, 0.5 to $1.5 \mathrm{~cm}$, white, slightly hard to hard; gradual smooth.

BCkb 144-173 cm; brown (7.5YR 5/4) clay; weak coarse prismatic; very hard; 25\% yellowish brown (5YR 5/ 4) pockets; $5 \%$ disseminated carbonate soft masses; clear smooth.

Bk1b 173-203 cm; brown (7.5YR 5.5/4) clay; weak coarse prismatic; very hard; $10 \%$ carbonate nodules, 0.5 to $1.5 \mathrm{~cm}$, white, slightly hard to hard; gradual smooth. Bulk humate radiocarbon sample of $9260 \pm 70$ B.P.

Bk2b 203-234 cm; light brown (7.5YR 6/4) clay; weak coarse prismatic; very hard; $15 \%$ carbonate nodules, 0.5 to $1.5 \mathrm{~cm}$, white, slightly hard to hard; few fine organic root pores; gradual smooth.

Bk3b 234-326 cm; light brown (7.5YR 6/4) clay; weak coarse prismatic; very hard; 40\% carbonate nodules, 0.5 to $1.5 \mathrm{~cm}$, white, slightly hard to hard; clear smooth.

$\mathrm{Cb} \quad 326-345 \mathrm{~cm}$; brown (7.5YR 5/4) clay; $10 \%$ light brown (7.5YR 6/3) pockets; massive; very hard; $2 \%$ carbonate clasts, $0.5 \mathrm{~cm}$, white, hard.

BHT-3; Early Holocene alluvium (EH); Holocene flood terrace $(6.5 \mathrm{~m})$; calcareous throughout.

A1 2-6 cm; very dark gray (10YR 3/1) clay loam; moderate medium subangular blocky; hard; common fine distinct strong brown (7.5YR 5/6) pore linings; gradual smooth.

A2 6-28 cm; very dark grayish brown (10YR 3.5/2) clay loam; moderate medium subangular blocky; friable; gradual smooth.

Bw 28-70 cm; brown (10YR 5.5/3) clay loam; moderate medium subangular blocky; firm; few very dark gray (10YR 3/1) biocasts; clear smooth.

Bk1 70-91 cm; yellowish brown (10YR 5/4) clay loam; few fine faint yellowish brown (10YR 5/8) pore linings; moderate medium angular blocky; hard; $2 \%$ carbonate filaments; gradual smooth. 
Bk2 91-118 cm; brown (10YR 5.5/4) clay loam; moderate medium angular blocky; hard; 3\% carbonate nodules, 0.5 to $1 \mathrm{~cm}$, white, slightly hard to hard; $3 \%$ pebbles, 1 to $2 \mathrm{~cm}$, subrounded, moderately sorted, matrix supported; gradual smooth.

Bk3 118-161 cm; dark grayish brown (10YR 4/2) and brownish yellow (10YR 6/6) clay loam; moderate medium angular blocky; hard; $10 \%$ pebbles, 1 to $3 \mathrm{~cm}$, subrounded, moderately sorted, matrix supported; $2 \%$ carbonate nodules, white, slightly hard to hard; clear wavy.

Bk4 161-198 cm; light brownish gray (10YR 6/2) clay loam; weak coarse angular blocky; very hard; $10 \%$ dispersed carbonate masses, 0.3 to $0.5 \mathrm{~cm}$; abrupt wavy.

Bkmb 198-210 cm; many cemented waterworn pebbles and cobbles, 2 to $10 \mathrm{~cm}$, subrounded, moderately sorted.

BHT-4; Late Holocene (LH) over Early Holocene alluvium (EH); Holocene flood terrace (6.5 m); calcareous throughout.

A $\quad$-14 cm; very dark gray (10YR 3.5/1) silty clay loam; moderate fine and medium subangular blocky; friable; $10 \%$ strong brown $(7.5 \mathrm{YR} 4 / 6)$ iron pore linings; gradual smooth.

BA 14-30 cm; dark gray (10YR 4/1) silty clay loam; common fine distinct strong brown (7.5YR 4/6) iron pore linings; moderate medium subangular blocky; friable; gradual smooth.

Bw1 30-63 cm; brown (10YR 4/2.5) clay loam; common fine distinct strong brown $(7.5 \mathrm{YR} 4 / 6)$ iron pore linings; moderate medium angular blocky; hard; gradual smooth.

Bw2 63-117 cm; grayish brown (10YR 4.5/2) clay loam; moderate medium angular blocky; hard; few fine faint dark yellowish brown (10YR 4/6) soft iron masses; $1 \%$ carbonate filaments; clear smooth.

Bw1b 117-150 cm; dark grayish brown (3.5/2) clay; 30\% brown (10YR 5/3); moderate coarse prismatic; very hard; gradual smooth.

Bw2b 150-184 cm; brown (7.5YR 5/3) clay; moderate coarse prismatic; very hard; gradual smooth.

Bkb 184-218 cm; brown (7.5YR 5/3) and light brown (7.5YR 6/3) clay; moderate coarse prismatic; very hard; $1 \%$ carbonate nodules, 0.5 to $1 \mathrm{~cm}$, white, hard; gradual smooth.

BC1b 218-272 cm; brown (7.5YR 5/4, 4/2) clay; moderate coarse prismatic; very hard; clear smooth.

BC2b 272-321 cm; brown (7.5YR 4.5/4, 4/2) clay; moderate coarse prismatic; very hard; $1 \%$ carbonate nodules, $0.5 \mathrm{~cm}$, white, hard; $1 \%$ pebbles, subrounded, $1 \mathrm{~cm}$, matrix supported; clear smooth. Bulk humate radiocarbon age of $7340 \pm 40$. 
$\mathrm{Cgb} \quad 321-364 \mathrm{~cm}$; light gray (10YR 7/2) and light yellowish brown (7.5YR 6/4) loam; common medium distinct light bluish gray (5B 7/1) iron depletions; massive; very firm; $1 \%$ pebbles subrounded, $1 \mathrm{~cm}$, matrix supported; partially carbonate cemented in lower part.

41TG378 (TU1); Holocene colluvium (HO) over Permian bedrock; upland deflational hillslope; calcareous throughout.

Bw1 0-15 cm; brown (7.5YR 5/4) sandy clay loam; moderate mediums subangular blocky; friable; few fine distinct brown ( $7.5 \mathrm{YR} 4 / 4)$ iron pore coats; $2 \%$ carbonate clasts 0.2 to $0.4 \mathrm{~cm}$, white, hard, angular; many fine roots; clear smooth.

Bw2 15-29 cm; brown (7.5YR 5/4) sandy clay loam; moderate medium subangular blocky; friable; few fine distinct brown (7.5YR 4/4) iron pore coats; $15 \%$ carbonate clasts 0.3 to $1 \mathrm{~cm}$, white, hard, angular; few large rip up carbonate clasts, 0.5 to $3 \mathrm{~cm}$, angular, white, very hard; common fine roots; clear smooth.

Bw3 29-38 cm; brown (7.5YR 5/4) clay loam; 30\% light reddish brown (2.5YR 6/4); moderate medium subangular blocky; firm; few fine roots; abrupt wavy.

$\mathrm{C} / \mathrm{Bb} \quad 38-54 \mathrm{~cm}$; faint bedding planes, pale yellow (5Y 8/2), 30\% brown (7.5YR 5/4), 5\% light reddish brown (2.5YR 6/4) silt loam; massive to weak coarse subangular blocky; hard; few fine roots; abrupt smooth.

$\mathrm{Crb} \quad 54-95 \mathrm{~cm}$; multiple bedding planes, 1 to $5 \mathrm{~cm}$, pale yellow (5Y 8/2), 30\% brown (7.5YR 5/4), 5\% light reddish brown (2.5YR 6/4), olive yellow (2.5Y 6/6), greenish gray (10GY 6/1) silt loam; few thin nodular iron zones.

$\underline{41 T G 389 \text { (TU1); Holocene eolian undifferentiated (HO); upland hillslope; calcareous throughout. }}$

A $\quad$ 0-12 cm; brown (10YR 4/3) fine sandy loam; few fine distinct brown (7.5YR 4/4/) iron pore linings; moderate medium subangular blocky; friable; many fine roots; gradual smooth.

Bw 12-29 cm; brown (10YR 4/3) fine sandy loam; few fine distinct brown (7.5YR 4/4) iron pore linings; moderate medium subangular blocky; friable; many fine roots; $2 \%$ carbonate clasts, angular, 0.2 to 0.3 $\mathrm{cm}$; clear smooth.

Bw 29-59 cm; brown (10YR 4/3) sandy clay loam; 15\% brown (10YR 5/3); common fine distinct brown (7.5YR 4/4) iron pore linings; moderate medium and coarse subangular blocky; firm; many fine roots; abrupt wavy.

Bkmb 59-63 cm; carbonate cemented channel gravels.

41TG410 (TU1); late Holocene alluvium (LH); Holocene flood terrace (6.5 m); calcareous throughout.

A1 0-11 cm; very dark grayish brown (10YR 3/2) silt loam; moderate fine and medium subangular blocky; firm; many roots and worm casts; gradual smooth. 
A2 11-26 cm; dark grayish brown (10YR 4/2) silt loam; moderate medium subangular blocky; firm; many fine and medium roots and worm casts; gradual smooth.

Bw1 26-45 cm; dark grayish brown (10YR 4/2) silt loam; moderate medium angular blocky; firm; many fine and medium roots and worm casts; gradual smooth.

Bw2 45-66 cm; brown (10YR 5/3) loam; moderate coarse angular blocky; hard; common fine and medium roots and worm casts; $1 \%$ carbonate filaments; gradual smooth.

Bw3 66-95 cm; brown (10YR 5/3) loam; weak coarse prismatic; hard; few fine and medium roots and worm casts; $1 \%$ carbonate filaments.

\section{South Concho River}

CB6; Early Holocene alluvium (EH); Holocene flood terrace $(6.5 \mathrm{~m})$; calcareous throughout. Quarry pit with $1.5 \mathrm{~m}$ of overburden.

A $\quad 0-35 \mathrm{~cm}$; very dark grayish brown (10YR 3/2) silty clay loam; moderate medium subangular blocky; very hard; gradual smooth.

AB $\quad 35-72 \mathrm{~cm}$; brown (10YR 4.5/3) silty clay loam; moderate coarse subangular blocky; firm; gradual smooth.

Bk 72-142 cm; brown (10YR 4/3) silty clay loam; 15\% brown (7.5YR 5/4); moderate medium angular blocky; firm; $1 \%$ carbonate filaments; $5 \%$ pebbles, 0.5 to $1.5 \mathrm{~cm}$, subrounded, matrix supported, moderately sorted; $6 \mathrm{~cm}$ pebble line at base of horizon; abrupt smooth.

BC1 142-178 cm; light brown (7.5YR 6/4) silty clay loam; weak coarse subangular blocky; firm; 3\% pebbles, 0.5 to $1.5 \mathrm{~cm}$, subrounded, matrix supported, moderately sorted; gradual smooth.

BC2 178-269 cm; strong brown (7.5YR 5/6) silty clay loam; 20\% light yellowish brown (10YR 6/4); weak coarse subangular blocky; firm; gradual smooth.

BC3 269-336 cm; light yellowish brown (10YR 6.5/4) sandy clay loam; massive; friable; abrupt wavy.

C $\quad 336-466 \mathrm{~cm} ; 65 \%$ pebbles, 0.5 to $5 \mathrm{~cm}$, subrounded, grain supported, moderately sorted.

CB7; Modern alluvium (MO) over Late Holocene alluvium (LH) over Early Holocene alluvium (EH); Holocene flood terrace $(6.0 \mathrm{~m})$; calcareous throughout. Quarry pit exposure.

A $\quad 0-13 \mathrm{~cm}$; very dark grayish brown (10YR 3/2) silty clay loam; moderate medium subangular blocky; hard; clear smooth.

Ab1 13-49 cm; black (10YR 2/1) silty clay loam; moderate medium subangular blocky; hard; abrupt wavy. 
Bwb1 49-111 cm; very dark gray (10YR 2.5/1) loam; 50\% pebbles, 1-8 cm, subrounded, matrix and grain supported, moderately sorted; abrupt wavy.

Cb1 111-158 cm; dark grayish brown (10YR 4/2) loam; 50\% pebbles, 1-8 cm, subrounded, matrix and grain supported, moderately sorted; abrupt wavy.

Bkb1 158-203 cm; brown (3.5/3) clay loam; moderate medium prismatic; very hard; $1 \%$ carbonate filaments; abrupt wavy. Bulk humate radiocarbon age of $3440 \pm 50$ в.P.

Cb1 203-277 cm; 70\% bedded pebbles, 1 to $6 \mathrm{~cm}$, subrounded, matrix and grain supported, moderately sorted; brown (10YR 4/3) loamy; abrupt wavy.

Bkb2 277-300 cm; brown (7.5YR 5/3) clay loam; few medium distinct strong brown (7.5YR 5/6) soft iron masses; $10 \%$ carbonate filaments.

\section{Spring Creek}

CB5; Late Holocene alluvium (LH); Holocene flood terrace (4.5 m); calcareous throughout.

A $\quad$-18 cm; very dark gray (10YR 3.5/1) clay loam; moderate medium and coarse subangular blocky; hard; common uncoated sand grains; abrupt smooth.

C1 18-31 cm; very dark gray (10YR 3/1) clay loam; $50 \%$ bedded pebbles, 0.3 to $0.6 \mathrm{~cm}$ and 1 to $4 \mathrm{~cm}$, poorly sorted, subrounded, grain supported; abrupt smooth.

C2 31-49 cm; very dark gray (10YR 3/1) clay loam; moderate medium subangular blocky; hard; occasional discontinuous pebble beds, 0.3 to $0.6 \mathrm{~cm}$ and 1 to $4 \mathrm{~cm}$, poorly sorted, subrounded, grain supported; abrupt wavy.

C3 49-134 cm; very dark gray (10YR 3.5/1) clay loam; 60\% pebbles, matrix and grain supported, 1 to $2 \mathrm{~cm}$ and 3 to $10 \mathrm{~cm}$, subrounded, moderately to moderately well sorted; abrupt wavy.

ABkb 134-153 cm; dark grayish brown (10YR 4/2.5) clay; moderate medium prismatic; very hard; $2 \%$ carbonate filaments; gradual smooth.

$\mathrm{Bk} / \mathrm{Cb}$ 153-228 cm; brown (7.5YR 4/3) clay; $2 \%$ carbonate filaments; $10 \mathrm{~cm}$ pebble bed in middle, $60 \%, 1-5$ $\mathrm{cm}$, subrounded, moderately well sorted, grain supported; abrupt wavy.

C1b 228-276 cm; brown (7.5YR 4/3) clay loam; multiple beds of pebbles, 0.2 to $0.4 \mathrm{~cm}$ and 0.5 to $1.5 \mathrm{~cm}$, subrounded, moderately to poorly sorted, grain supported; abrupt wavy.

C2b 276-301 cm; 65\% pebbles, 1 to $7 \mathrm{~cm}$, moderately sorted, subrounded, grain supported; loamy brown matrix; abrupt wavy.

C3b 301-330 cm dark grayish brown (10YR 4/3.5) clay; massive; very firm. 
C4b 330-380 cm; $65 \%$ pebbles, 1 to $6 \mathrm{~cm}$, subrounded, grain supported, moderately sorted; brown loamy matrix; abrupt wavy.

C5b 380-400 cm; brown (10YR 4/3) clay loam; 2\% carbonate filaments; massive; firm. Bulk humate radiocarbon age of $3460 \pm 60$ B.P.

BHT-8; Late Pleistocene alluvium (LP1), late Pleistocene terrace; calcareous throughout.

A $\quad 0-17 \mathrm{~cm}$; very dark grayish brown (10YR 3/2) clay loam; moderate medium subangular blocky; firm; common fine distinct brown (7.5YR 4/4) iron pore linings; gradual smooth.

Bw $\quad$ 17-37 cm; brown (7.5YR 5/4) silty clay loam; few fine faint brown (7.5YR 4/4) iron pore linings; weak medium subangular blocky; friable; abrupt irregular.

Bk1 37-83 cm; light brown (7.5YR 6/4) silty clay loam; common fine distinct yellowish brown (10YR 5/6) soft iron masses; $50 \%$ coalescing soft to hard carbonate masses; moderate medium angular blocky; hard; abrupt wavy.

Bk2 83-150 cm; brown (7.5YR 5/4) clay; few biocasts; moderate coarse angular blocky; hard; 20\% carbonate nodules, 1 to $2 \mathrm{~cm}$, slightly hard to hard, white; gradual smooth.

BCk3 150-197 cm; light brown (7.5YR 6/4) silty clay loam; moderate coarse prismatic; very hard; $1 \%$ carbonate clasts, $1 \mathrm{~cm}$, subrounded; common organic/manganese root linings.

BHT-9; truncated version of BHT-8.

BHT-10; Early Holocene alluvium (EH); Holocene flood terrace $(6.5 \mathrm{~m})$; calcareous throughout.

Bk 0-49 cm; brown (7.5YR 5/4) clay loam; common fine distinct dark yellowish brown (10YR 4/6) iron pore linings; moderate to strong medium angular blocky; very hard; $3 \%$ carbonate filaments; gradual smooth.

Bw1 49-120 cm; light reddish brown (5YR 6/4) clay loam; weak coarse prismatic; very firm; gradual smooth.

Bw2 120-210 cm; pink (7.5YR 7/4) loam; common medium distinct light gray (5Y 7/1) iron depletions; weak coarse prismatic; very firm; gradual smooth.

Bw3 210-265 cm; pink (7.5YR 7/4) loam; 20\% brown (7.5YR 5/4); common medium distinct light gray (5Y 7/1) iron depletions; weak coarse prismatic; very firm; gradual smooth. 


\section{Unnamed/No Name Tributary}

CB4; Late Holocene alluvium (LH) over early Holocene alluvium (EH) over Permian bedrock; Holocene flood terrace $(3 \mathrm{~m})$; calcareous throughout.

A $\quad 0-30 \mathrm{~cm}$ brown (7.5YR 5/4) clay loam; moderate medium subangular blocky; firm; few fine distinct strong brown (7.5YR 5/6) iron pore linings; clear smooth. (lake deposit)

A1 30-46 cm; very dark grayish brown (10YR 3/2) clay loam; few fine distinct dark yellowish brown (10YR 4/6) iron pore linings; strong fine and medium angular blocky; very firm; gradual smooth.

A2 46-71 cm; dark grayish brown (10YR 4/2) clay loam; few fine distinct yellowish brown (10YR 5/8) iron pore linings; moderate medium angular blocky; very firm; gradual smooth.

Bk 71-107 cm; brown (10YR 5/3) clay loam; moderate medium and coarse angular blocky; very firm; few fine distinct yellowish brown (10YR 5/8) iron pore linings; $2 \%$ carbonate nodules, white, hard, 0.5 to $1.5 \mathrm{~cm}$; clear smooth.

Abkb1 107-142 cm; brown (7.5YR 4/3) clay loam; many distinct clay/organic ped coats; moderate medium angular blocky; very firm; $5 \%$ carbonate nodules, white, hard, 0.5 to $1.5 \mathrm{~cm}$; gradual smooth.

Bkb1 142-169 cm; brown (7.5YR 4/4) clay loam; common distinct clay/organic ped coats; moderate medium angular blocky; very firm; $12 \%$ carbonate nodules, 0.5 to $2 \mathrm{~cm}$, white, hard; clear smooth.

Bw1b1 169-215 cm; strong brown (7.5YR 5/8) clay loam; weak medium subangular blocky; firm; clear smooth.

Bw2b1 215-248 cm; strong brown (7.5YR 5/6) sandy clay loam; weak coarse angular blocky; firm; gradual smooth.

B/Cb1 248-297 cm; multiple beds 2 to $10 \mathrm{~cm}$ of pink (7.5YR 8/4) and strong brown (7.5YR 5/6) fine sandy loam; weak coarse subangular blocky; friable; gradual smooth.

Cb1 297-325 cm; 70\% pebbles, 0.2 to $0.5 \mathrm{~cm}$, well sorted, subrounded, grain supported.

Crb2 $325 \mathrm{~cm}$; yellow and olive sandstone bedrock.

BHT-5; Late Holocene alluvium (LH); Holocene flood terrace $(3 \mathrm{~m})$; calcareous throughout.

A $\quad 0-12 \mathrm{~cm}$; very dark gray (10YR 3.5/1) clay loam; moderate fine and medium subangular blocky; friable; $5 \%$ dark yellowish brown (10YR 4/4) iron pore linings; gradual smooth.

BA $\quad 12-28 \mathrm{~cm}$; dark grayish brown (10YR 4/2) clay loam; moderate medium subangular blocky; friable; $8 \%$ dark yellowish brown (10YR 4/4) iron pore linings; many very dark gray (10YR 3/1) biocasts; gradual smooth.

Bw1 28-70 cm; brown (7.5YR 4.5/4) clay loam; 5\% dark yellowish brown (10YR 4/6) iron pore linings; common very dark gray (10YR 3/1) biocasts; moderate medium and coarse subangular blocky; firm; gradual smooth. 
Bw2 70-101 cm; brown (7.5YR 5/4) clay loam; common very dark gray (10YR 3/1) biocasts; moderate medium angular blocky; firm; gradual smooth.

Bw3 101-153 cm; strong brown (7.5YR 5.5/6) clay loam; few very dark gray (10YR 3/1) biocasts; few fine dark gray (10YR 4/1) pore iron depletions; moderate medium and coarse angular blocky; very firm; clear smooth.

By 153-230 cm; brown (7.5YR 5/4) clay loam; few fine dark gray (10YR 4/1) pore iron depletions; moderate medium and coarse angular blocky; very firm; $5 \%$ gypsum crystals 0.2 to $0.3 \mathrm{~cm}$.

Estimated description from extended trench.

230-310 cm; 60\% pebbles, 1 to $8 \mathrm{~cm}$, subrounded, moderately to poorly sorted, matrix supported; yellowish brown (10YR 5/4) clay loam matrix.

310-340 cm; brownish yellow (10YR 6/6) very fine sandy loam; 3\% dark yellowish brown (10YR 4/4) iron pore linings; $2 \%$ light gray (10YR 7/1) iron depletions.

BHT-6; Late Holocene alluvium (LH) over early Holocene alluvium (EH); Holocene flood terrace (3 m); calcareous throughout.

C $\quad 0-8 \mathrm{~cm}$; light gray (2.5Y 7/1) fine sandy loam; single grained; few fine faint yellowish brown (10YR 5/ 6) iron pore linings; abrupt wavy. (lake deposit).

A1 8-33 cm; very dark gray (10YR 2.5/1) clay loam; moderate medium subangular blocky; friable; few fine faint dark yellowish brown (10YR 3/4) iron pore linings; gradual smooth.

A2 33-51 cm; very dark gray (10YR 2.5/1) clay loam; moderate medium subangular blocky; friable; common fine distinct yellowish brown (10YR 5/6) iron pore linings; gradual smooth.

Bw1 51-76 cm; very dark grayish brown (10YR 3.5/2) clay loam; moderate medium angular blocky; hard; $15 \%$ dark yellowish brown (10YR 3/4) iron pore linings; $2 \%$ carbonate filaments; $5 \%$ pebbles, 0.2 to $0.4 \mathrm{~cm}$, subrounded, moderately sorted; gradual smooth.

Bw2 76-103 cm; yellowish brown (10YR 4.5/4) clay loam; 5\% strong brown (7.5YR 5/6) pore linings; moderate medium angular blocky; $10 \%$ pebbles, 0.2 to $0.8 \mathrm{~cm}$, subrounded, moderately sorted, matrix supported; $2 \%$ carbonate filaments; abrupt wavy.

$\mathrm{ABb} \quad 103-118 \mathrm{~cm}$; brown (7.5YR 4/3) clay; 5\% very dark gray (10YR 3/1) biocasts; moderate coarse angular blocky; very hard; $1 \%$ carbonate filaments; common distinct clay/organic ped coats; gradual wavy. Bulk humate radiocarbon age of $3360 \pm 50$ B.P.

Bkb 118-150 cm; brown (7.5YR 4/4) clay; few very dark gray (10YR 3/1) biocasts; moderate medium and coarse prismatic; very hard; $5 \%$ carbonate nodules, $1 \mathrm{~cm}$, white, hard; common distinct clay/organic ped coats; gradual wavy. 
Bwb 150-199 cm; brown (7.5YR 4/3) clay; few very dark gray (10YR 3/1) biocasts; common medium distinct dark gray (10YR 4/1) iron depletions; moderate coarse prismatic; very hard; common distinct clay/ organic ped coats; clear wavy.

Byb 199-221 cm; brown (7.5YR 4/3, 10YR 5/3) clay; 2\% gray (10YR 5/1) iron depletions; moderate medium prismatic to moderate medium angular blocky; firm; $10 \%$ gypsum crystals, 0.2 to $0.4 \mathrm{~cm}$; abrupt wavy.

$\mathrm{Cb} \quad 221-241 \mathrm{~cm}$; brownish yellow (10YR 6/6) sandy clay; 20\% light yellowish brown (10YR 6/4); few gray (2.5Y 6/1) iron depletions; massive; very firm; few fine carbonate nodules.

BHT-7; Late Pleistocene alluvium (LP1); Late Pleistocene terrace (5 m); calcareous throughout. Deflated surface by 20 to $30 \mathrm{~cm}$.

C $\quad 0-10 \mathrm{~cm}$ light gray $(2.5 \mathrm{Y} 7 / 2)$ fine sand; single grained; few fine faint brown $(7.5 \mathrm{YR} 4 / 4)$ iron pore linings; abrupt wavy. (lake deposit)

A $\quad 10-22 \mathrm{~cm}$; yellowish brown (10YR 5/4) sandy clay loam; weak medium to coarse subangular blocky; friable; $1 \%$ carbonate nodules, 0.5 to $1 \mathrm{~cm}$, white, hard; clear wavy.

ABk 22-41 cm; reddish yellow (7.5YR 6/6) sandy clay loam; 20\% brown (7.5YR 5/4); weak coarse subangular blocky; friable; $10 \%$ carbonate nodules, 0.5 to $1 \mathrm{~cm}$, white, hard; gradual wavy.

Bk1 41-70 cm; reddish yellow (7.5YR 6/6) sandy clay loam; 5\% strong brown (7.5YR 5/6) iron soft masses; weak coarse subangular blocky; friable; $8 \%$ carbonate nodules, 0.5 to $1 \mathrm{~cm}$, white, hard; gradual wavy.

Bk2 70-112 cm; strong brown (7.5YR 5.5/6) fine sandy loam; 5\% strong brown (7.5YR 5/6) iron soft masses; weak coarse angular blocky; firm; $15 \%$ carbonate nodules, 0.5 to $1.5 \mathrm{~cm}$, white, hard; gradual wavy.

Bk3 112-151 cm; reddish yellow (7.5YR 6.5/6) fine sandy loam; 40\% light brown (7.5YR 6/3); weak coarse prismatic; hard; $10 \%$ carbonate nodules, 0.5 to $1.5 \mathrm{~cm}$, white, hard; gradual wavy.

BC 151-174 cm; yellowish red (5YR 5/6) and pale brown (7.5YR 6/3) fine sandy loam; weak coarse prismatic; hard; $1 \%$ carbonate nodules, $0.5 \mathrm{~cm}$, white, hard; abrupt wavy.

C 174-200 cm; gravely and sandy bedded with common laminations; gravel beds- $60 \%$ pebbles, 0.2 to 0.5 $\mathrm{cm}$, subrounded, moderately well sorted, grain supported; sandy beds-light brown (7.5YR 6.5/4). Permian bedrock at base. 


\title{
Appendix J: Twin Buttes Archaeological Project
}

\section{Paleomagnetic and Magnetic Soil Susceptibility Results}

\author{
by Wulf Gose
}

\section{Introduction}

This appendix presents the results of soil susceptibility sampling from 41TG378, 41TG389, and 41TG410, and the results of archaeomagnetic samples collected from a fire-cracked rock feature at 41TG389. A brief background of these analyses is given, followed by specific site analyses. The reader is referred to Chapter 6 for a discussion of the methods used to process the samples.

\section{Soil Susceptibility Analysis}

\section{Background}

The magnetic susceptibility (MS) is a parameter which is proportional to the magnetic mineral content of a rock or soil sample. If the sample contains only one magnetic mineral, then the susceptibility is a direct measure of the concentration of this mineral. The susceptibility of a natural soil can be modified by pedogenic as well as cultural processes. Both processes tend to enhance the MS. In the organic-rich top layer of a soil, the susceptibility increases by as much as a factor of ten, probably due to the creation of maghemite as a result of organic activities (e.g., Singer and Fine 1989). If the soil is subsequently buried, this increase is preserved and can be used to identify paleosols.

The susceptibility is also enhanced by the action of fire. In the reducing environment of a wood-burning fireplace, new magnetic minerals are formed such as magnetite or maghemite. This effect can be observed in rocks and soils, as well as in the ash from the wood (Gose 2000; McClean and Kean 1993; Tite and Mullins 1971). The heat and ash produced by a grass fire are insufficient to enhance the MS.

\section{Site Analysis}

\section{$41 T G 378$}

Samples for magnetic susceptibility measurements were collected in $3-\mathrm{cm}$ intervals down to $100 \mathrm{~cm}$ below datum. The susceptibilities are very low, close to the detection limit of the instrument. The profile shows two distinct zones; from the surface to a depth of about $48 \mathrm{~cm}$ and from $62 \mathrm{~cm}$ to $100 \mathrm{~cm}$ (Figure J-1). The intervening interval is a transition zone. No obvious expressions of human activity can be discerned. The two susceptibility units correspond well with the observed lithology which shows Permian strata overlain by Holocene beds (see Chapter 4). 


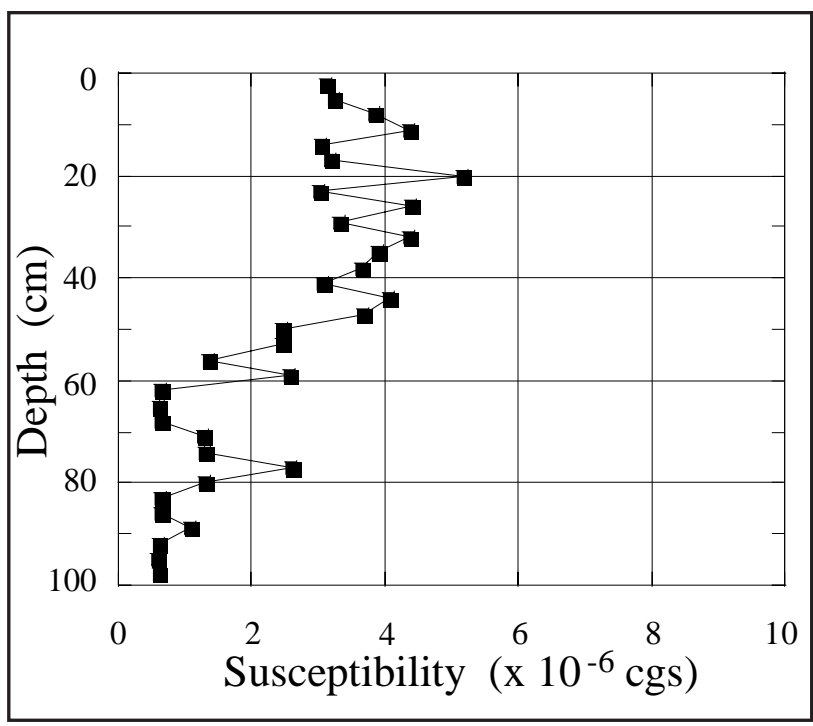

Figure J-1. Soil susceptibility profile of Unit 1, $41 T G 378$.

\section{$41 T G 380$}

Profiles $31 \mathrm{E}$ and $31 \mathrm{~W}$ are very similar (Figures $\mathrm{J}-2$ and $\mathrm{J}-3$ ). Both show a decrease in susceptibility up-section. The two top samples in profile $31 \mathrm{~W}$ have much lower susceptibilities than the other samples and the sample at the 7.5$\mathrm{cm}$ depth in profile $31 \mathrm{E}$ is very large. These three samples seem to be "contaminated" or, in some way, not representative of these sections. The general upward decrease in susceptibility suggests an influx of material from a different source area. At the level where a burned rock feature was found, both profiles show one sample with larger susceptibility values (at $25 \mathrm{~cm}$ in profile $31 \mathrm{E}$ and at $40 \mathrm{~cm}$ in profile $31 \mathrm{~W}$ ). Such increases can be due to the presence of wood ash (e.g., Collins et al. 1994).

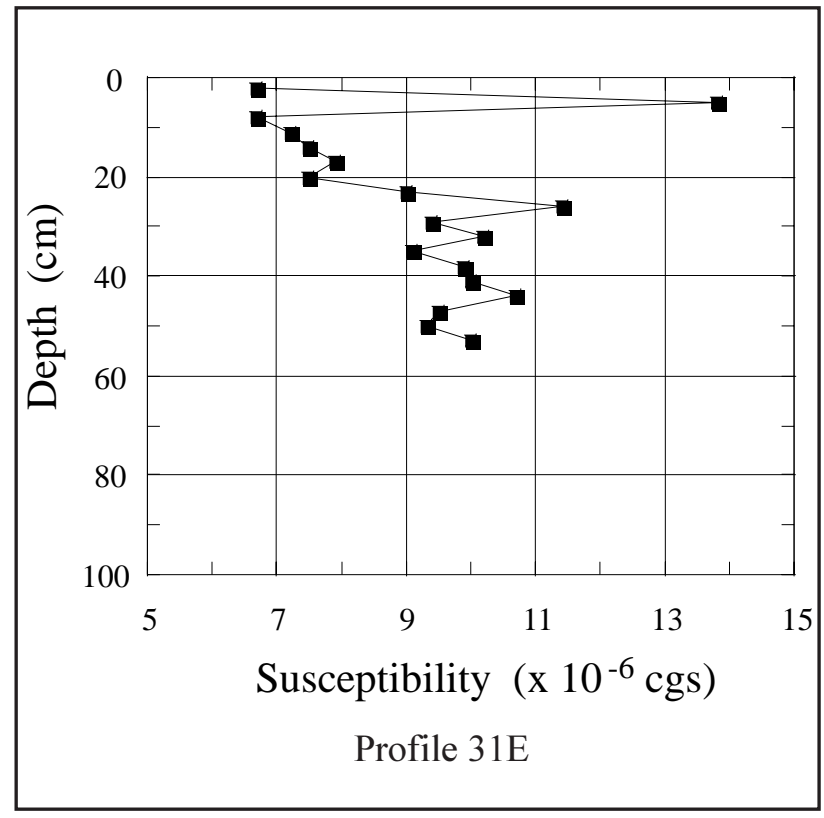

Figure J-2. Soil susceptibility profile of the east wall of Unit 1, 41TG389.

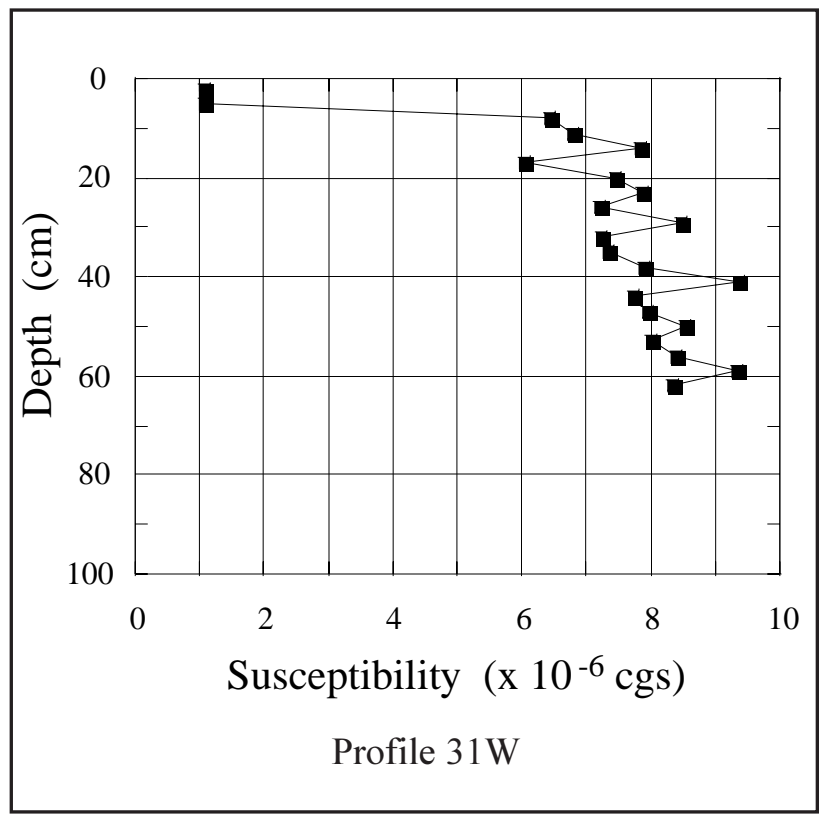

Figure J-3. Soil susceptibility profile of the west wall of Unit 1, 41TG389. 


\section{$41 T G 410$}

Samples from this one meter long profile (Figure J-4) yielded the largest susceptibility values of the four sites, averaging about $13 \times 10^{-6} \mathrm{cgs}$ (centimeter, gram, second) units. The variations with depth are small. In the interval from 30 to $50 \mathrm{~cm}$ or possibly $70 \mathrm{~cm}$ below datum the values are slightly larger. This may be the result of human activity, mainly the burning of wood.
Figure J-4. Soil susceptibility profile of Unit 1, $41 T G 410$.

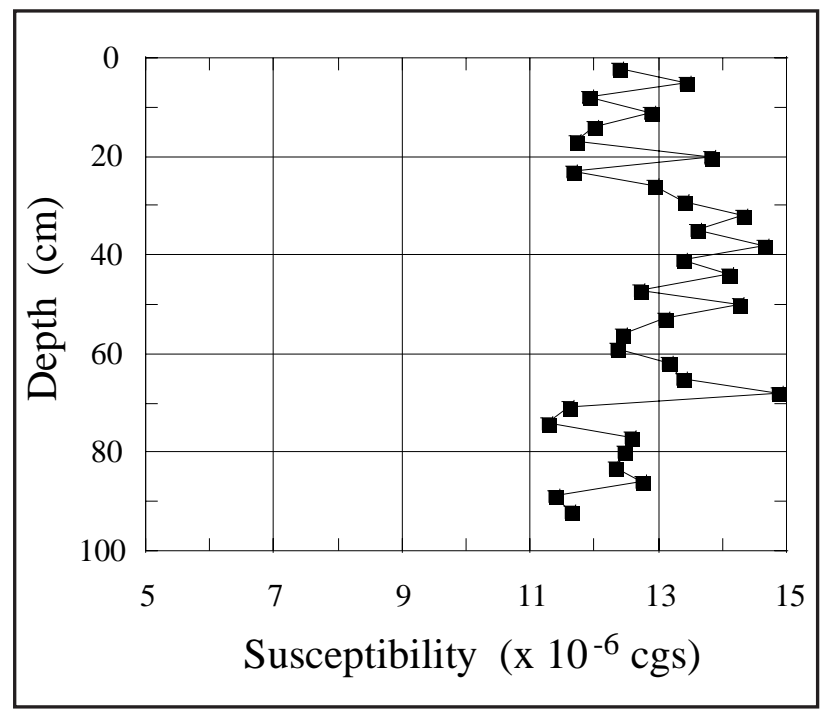

\section{Archaeomagnetic Analysis of Heated Rocks - 41TG389}

\section{Background}

Paleomagnetic methods can be applied to a variety of archaeological problems. The most common application of archaeomagnetism makes use of the secular variation of the earth's magnetic field for dating archaeological sites (e.g., Eighmy and Sternberg 1990). The material used in these studies typically is taken from clay-lined fire pits. As the fireplace cools to ambient temperature, the magnetic minerals in the clay liner will acquire a thermoremanent magnetization (TRM). The direction of this TRM will be parallel to the geomagnetic field as seen at this site and at this point in time. Similarly, if a stone is heated above the Curie temperature of its magnetic mineral(s), it will be magnetized parallel to the ambient magnetic field if it cools in situ. The Curie temperature is a mineral specific temperature above which all magnetic alignments are randomized and thus all prior magnetic information is lost. The value for magnetite is $580^{\circ} \mathrm{C}$ and $680^{\circ} \mathrm{C}$ for hematite. If, on the other hand, the stone is moved while cooling it will acquire partial thermoremanent magnetization (pTRM) components for each temperature interval during which the stone was not moved. If moved only once at temperature T1, say $300^{\circ} \mathrm{C}$, then the rock will have two components of magnetization, one corresponding to the time when the rock cooled from above its Curie temperature to $300^{\circ} \mathrm{C}$ and a second component which was acquired between $300^{\circ} \mathrm{C}$ and the ambient temperature. In the laboratory, these components can often be identified by detailed progressive demagnetization.

The paleomagnetic data are best viewed in a vector component diagram. Here, the magnetic vector is projected onto two perpendicular planes, typically the north-south-east-west plane and the up-down-horizontal plane, and the two graphs are combined into one figure. Each demagnetization step yields one point in each plane and the sequential data points are connected. A component of magnetization is identified by at least three colinear points. The data are analyzed in three dimensions by the principal component analysis (PCA, Butler 1992; Kirschvink 1980). PCA calculates the best-fitting vector over a selected demagnetization range. 
The resulting directions of magnetization are then plotted in a stereographic projection. The declination is counted clockwise starting at north and the inclination varies from $0^{\circ}$ at the periphery to $90^{\circ}$ at the center of the stereonet. The paleomagnetic methods as applied to archaeological samples has recently been reviewed by Gose (2000).

\section{Site Analysis}

Eleven burned rock samples from a fire-cracked rock feature at 41TG389 were subjected to paleomagnetic analyses. All samples were thermally demagnetized in $50^{\circ} \mathrm{C}$ increments from $150^{\circ} \mathrm{C}$ to $550^{\circ} \mathrm{C}$. All samples contained two components of magnetization as best seen in vector component diagrams for rocks $\mathrm{J}$, E, and $\mathrm{H}$ (Figure J-5). In these diagrams, the normalized intensity of magnetization is plotted for all demagnetization

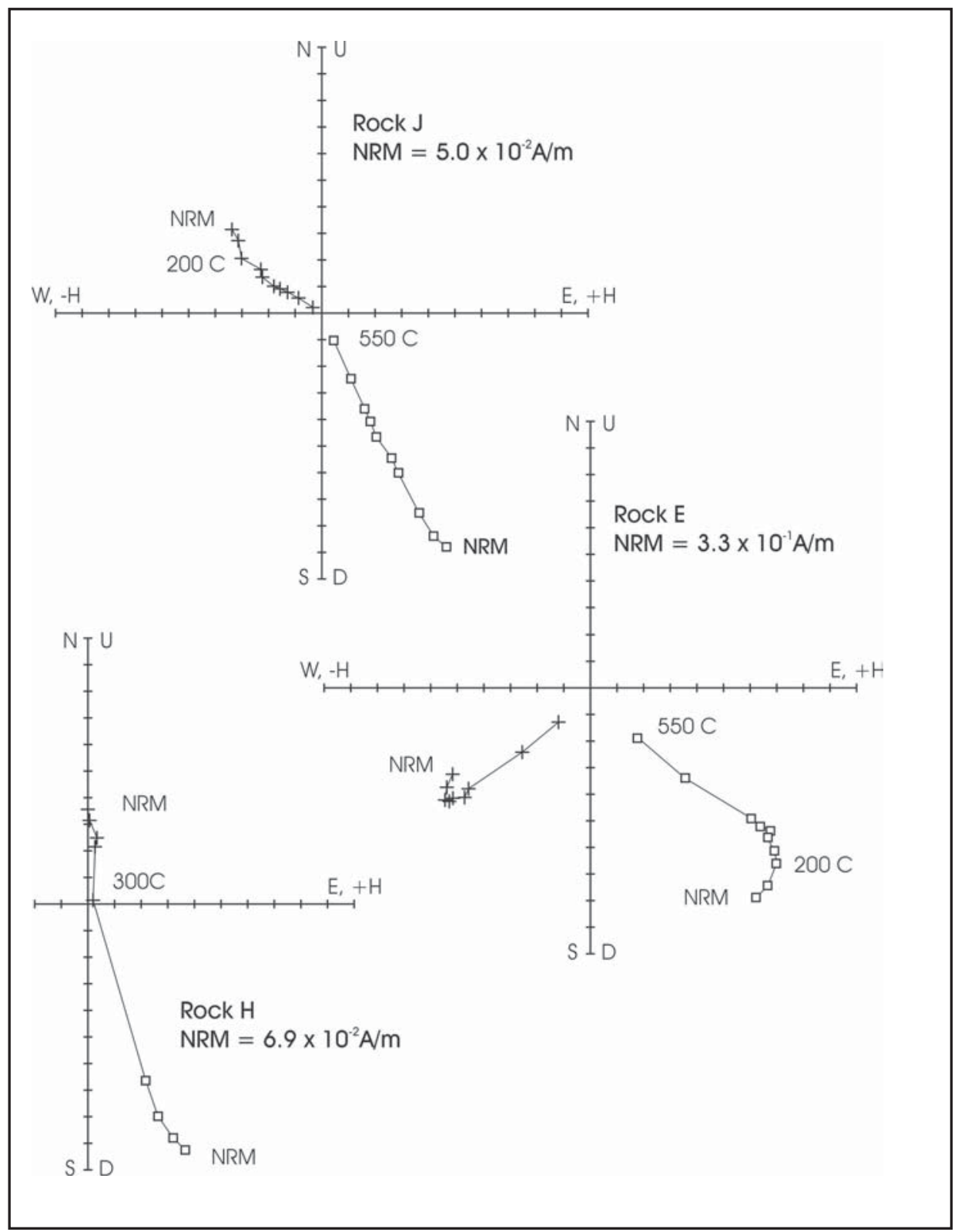

Figure J-5. Components of magnetization in vector component diagrams for rocks $J, E$, and $H$. 
steps in two perpendicular planes. A component of magnetization is identified by three or more colinear points and the best fitting vector is calculated by the principal component method (Kirschvink 1980). All samples contain a low-temperature component which is stable up to 200 or $250^{\circ} \mathrm{C}$ and a high temperature component which, in most cases, is stable up to $550^{\circ} \mathrm{C}$.

The directions of the best fitting vectors are plotted in a stereographic projection in Figure J-6. An examination of Figure J-6a indicates that all of the rocks contain a low temperature magnetization at $250^{\circ} \mathrm{C}$ which clusters near the present dipole field direction, except for rock I.

Sample I has a negative direction of magnetization implying that this rock was turned upside down after it cooled. When heated beyond $250^{\circ} \mathrm{C}$, the directions of the high temperature components scatter, representing the original, geological magnetization. These results imply that the rocks were heated to about $250^{\circ} \mathrm{C}$ and cooled, and have remained in situ since. Only rock I was turned over.

Figure J-6. Directions of the best fitting vectors in a stereographic projection for (a) low temperature, ca. $250^{\circ} \mathrm{C}$, and (b) high temperature, $>250^{\circ} \mathrm{C}$, representing the original, geological magnetization.

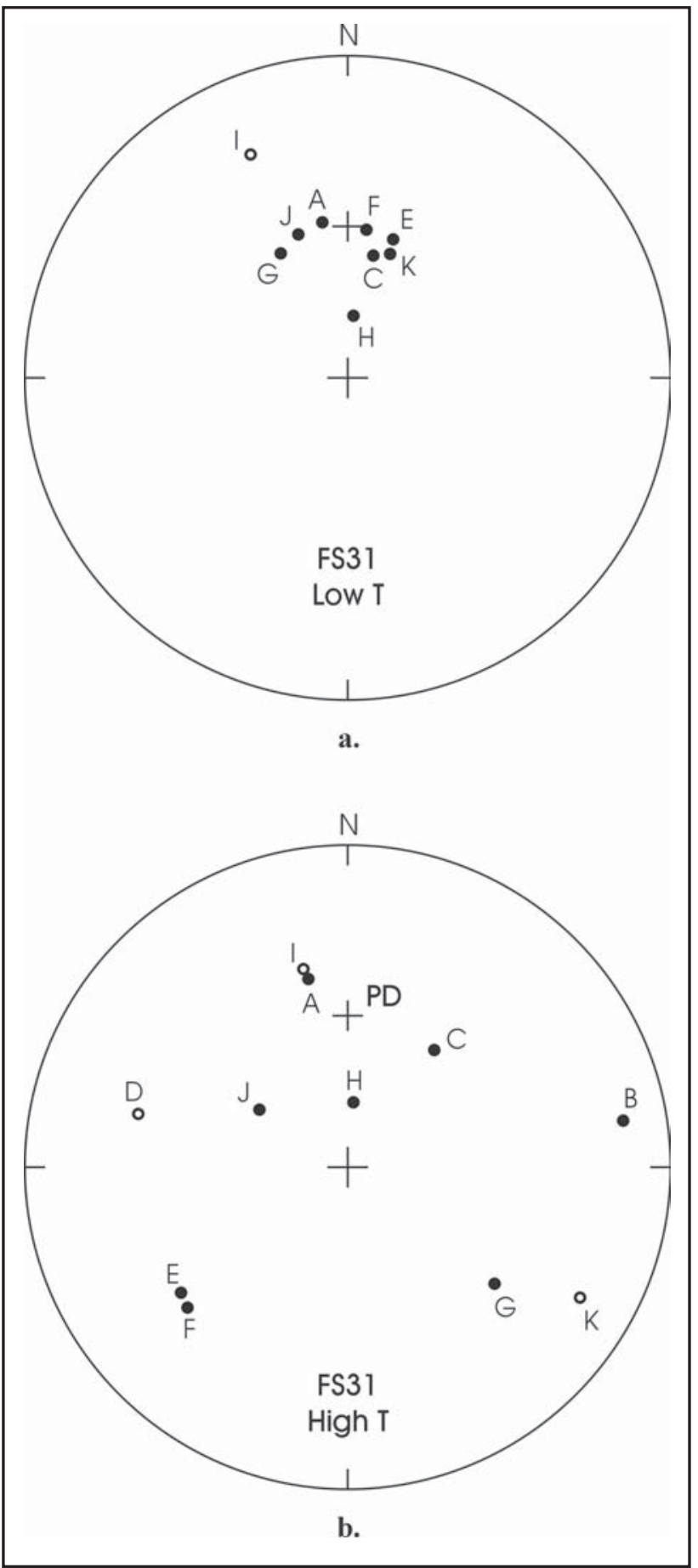




\section{References Cited}

Butler, R. F.

1992 Paleomagnetism: Magnetic Domains to Geologic Terranes. Blackwell Scientific Publications, Boston.

Collins, M. B., W. A. Gose, and S. Shaw

1994 Preliminary Geomorphological Findings at Dust and Nearby Caves. Journal of Alabama Archaeology 40:35-56.

Eighmy, J. L., and R. S. Sternberg (editors)

1990 Archeomagnetic Dating. University of Arizona Press, Tucson.

Gose, W. A.

2000 Paleomagnetic Studies of Burned Rocks. Journal of Archaeological Science 27:409-421.

Kirschvink, J. L.

1980 The Least Square Line and Plane and the Analysis of Paleomagnetic Data. Geophysical Journal of the Royal Astronomical Society 62:699-718.

McClean, R. G., and W. F. Kean

1993 Contributions of Wood Ash Magnetism to Archaeomagnetic Properties of Fire Pits and Hearths. Earth and Planetary Science Letters 119:387-394.

Singer, M. J., and P. Fine

1989 Pedogenic Factors Affecting Magnetic Susceptibility of Northern California Soils. Soil Science Society of America Journal 53:1119-1127.

Tite, M. S., and C. Mullins

1971 Enhancement of the Magnetic Susceptibility of Soils on Archeological Sites. Archaeometry 13:209-219. 


\section{Appendix K: \\ Twin Buttes Archaeological Project}

\section{Project Forms}

This appendix provides copies of all forms used on the Twin Buttes survey, along with information sheets.

1) Twin Buttes survey site checklist. Two pages.

2) Twin Buttes shovel test form, and explanation sheet. Two pages.

3) Isolated find log. One page.

4) Unique item log. One page.

5) Photographic log. One page.

6) Archaeomagnetic sample log. One page.

7) Unit level excavation form. Two pages.

8) Collection bag log. One page.

9) Surface observation area recording form and explanation. Three pages. 


\section{TWIN BUTTES SURVEY SITE CHECKLIST - Phase I}

Field Site \# Area Nearest Station and Transect

1. Identify surface artifact distribution, establish boundary, and then place site datum with 望 "iron rebar and aluminum identification tag.

2. Complete sketch map.

Indicate provenience of datum, features, and diagnostics collected. Map in artifact concentrations, disturbed areas, slope, approximate boundary, and natural terrain features such as drainages, etc.

3. Take GPS readings on site datum and boundary.

4. Complete the following checklist:

\begin{tabular}{||l|l|l||}
\hline \hline Artifacts & Present & Absent \\
\hline Cores & & \\
\hline Primary Flakes & & \\
\hline Secondary Flakes & & \\
\hline Tertiary Flakes & & \\
\hline Tested Cobbles & & \\
\hline Quarry Blanks & & \\
\hline Preforms & & \\
Bifaces & & \\
\hline Unifaces & & \\
\hline Retouched Flakes & & \\
\hline Utilized Flakes & & \\
\hline Ground Stone & & \\
\hline Fire Cracked Rock (Not \\
Modern) & & \\
\hline Prehistoric Hearth & & \\
\hline
\end{tabular}

Twin Buttes Survey Site Checklist form, page 1. 


\begin{tabular}{||l|l|l||}
\hline Prehistoric Ceramics & & \\
\hline Historic Ceramics or Glass & & \\
\hline Historic Metal & & \\
\hline Historic Structure & & \\
\hline
\end{tabular}

5. Make subjective and general observations about the site:

a. Setting (circle one) riverine, terrace, upland

b. Vegetation

c. Estimated surface visibility (\%)

d. Nearest extant water

e. Observable landmarks (and Degrees)

f. Subjective evaluation of disturbance which equates to \% of site still intact

g. Estimated debitage present (check mark on line):

$<10 \_>10 \_>50 \_>100 \_>150 \_>300 \_>500 \_$

h. Estimated fire cracked rock present (check mark on line): $<10 \_>10 \_>50 \_>100 \_>150 \_>300 \_>500 \_>1,000$

i. Subjective evaluation of artifacts observed on the surface expressed in numbers:

\# Tertiary Flakes

\# Primary Flakes

\# Secondary Flakes

\# Cores

\# Tested Cobbles

\# Bifaces

\# Unifaces

\# Retouched Flakes

\# Utilized Flakes

Diagnostics Collected:

Twin Buttes Survey Site Checklist form, page 2. 
Shovel Test \#

Date

EXCAVATOR(S)

0-10 CM

COLOR

TEXTURE

STRUCTURE

MOTTLES

INCLUSIONS

MATERIAL COLLECTED

REMAR KS

10-20 CM

COLOR

TEXTURE

STRUCTURE

MOTTLES

IN CLUSIONS

MATERIAL COLLECTED

REMAR KS

20-30 CM COLOR

TEXTURE

STRUCTURE

MOTTLES

IN CLUSIONS

MATERIAL COLLECTED

REMAR KS

30-40 CM COLOR

TEXTURE

STR UCTURE

MOTTLES

IN CLUSIONS

MATERIAL COLLECTED

REMAR KS

40-50 CM COLOR

TEXTURE

STRUCTURE

MOTTLES

INCLUSIONS

MATERIAL COLLECTED

REMAR KS

(See reverse side for soil description guidelines)

Twin Buttes shovel test form. 
1. Depth - top and bottom layer, centimeters from surface.

2. Color - Munsell Chart, moist or dry, but indicate which-be consistent!

\section{Texture -}

Type $=1 . \underline{\text { Sand }}$ 2. $\underline{\text { Sandy Loam }}$ 3. Loam 4. Silt Loam 5. Clay Loam 6. Clay Consistency $($ moist $)=$ Loose Friable Firm Extremely Firm

4. Structure-

Type: 1. Massive

Size $=$ fine $<2 \mathrm{~cm}$

2. Blocky

3. Prismatic

4. Granular

5. Platy

Grade $=$ weak

medium $<5 \mathrm{~cm}$

moderate coarse $>5 \mathrm{~cm}$ strong

5. Mottles - Color $=$ Munsell Chart

$\begin{array}{llll}\text { Abundance }= & \text { few }<2 \% & \text { common }<20 \% & \text { many }>20 \% \\ \text { Size }= & \text { fine }<0.5 \mathrm{~cm} & \begin{array}{l}\text { medium }<1.5 \mathrm{~cm} \\ \text { distinct }\end{array} & \text { coarse }>1.5 \mathrm{~cm} \\ \text { Contrast }= & \text { faint } & \text { prominent }\end{array}$

6. Inclusions - manganese, charcoal, calcium carbonate, pebbles-cobbles, roots, rodent burrows, leaf litter, artifacts, features, or anything else.

\section{Lower Boundary -}

Distinctness = very abrupt $<1 \mathrm{~mm} \quad$ abrupt $<2.5 \mathrm{~cm} \quad$ clear $<6 \mathrm{~cm}$ gradual $<12.5 \mathrm{~cm} \quad$ diffuse $>12.5 \mathrm{~cm}$

Topography $=$ smooth, wavy, irregular, broken, sloping, horizontal

8. Soil Horizon - O, A, B, E, C, R-if you are sure!

Field Definitions of Texture -

1. $\underline{\text { Sand }}$ - loose and single grained-moist squeezed cast will crumble

2. Sandy Loam - mostly sand with silt and clay_individual sand grains visible, moist cast can bear careful handling

3. Loam - even mixture of sand, silt, and clay_-gritty, but smooth and slightly plastic, moist cast handled freely

4. Silt Loam - fine sands, little clay, mostly silt_-dry clods break easily, soft, smooth and floury if dry, moist casts don't break, won't ribbon

5. Clay Loam - dry clods are hard, moist ribbon breaks easily, moist cast bears heavy handling, kneaded heavy compact mass that won't crumble

6. Clay - very hard clods, very plastic ad sticky if wet, flexible ribbon 
Center for Archaeological Research - UTSA

Twin Buttes Reservoir Survey

ISOL A TED FIND LOG

\begin{tabular}{|c|c|c|c|c|}
\hline Iso. Find\# & Area & Station & Description & Date \\
\hline & & & & \\
\hline & & & & \\
\hline & & & & \\
\hline & & & & \\
\hline & & & & \\
\hline & & & & \\
\hline & & & & \\
\hline & & & & \\
\hline & & & & \\
\hline & & & & \\
\hline & & & & \\
\hline & & & & \\
\hline & & & & \\
\hline & & & & \\
\hline & & & & \\
\hline & & & & \\
\hline & & & & \\
\hline & & & & \\
\hline & & & & \\
\hline & & & & \\
\hline & & & & \\
\hline & & & & \\
\hline & & & & \\
\hline
\end{tabular}

Twin Buttes Isolated Find log. 
Center for Archaeological Research - UTSA

Twin Buttes Reservoir Survey

UNIQUE ITEM LOG

\begin{tabular}{|c|c|c|c|c|}
\hline Unique \# & Area & Description & Surveyor & Date \\
\hline & & & & \\
\hline & & & & \\
\hline & & & & \\
\hline & & & & \\
\hline & & & & \\
\hline & & & & \\
\hline & & & & \\
\hline & & & & \\
\hline & & & & \\
\hline & & & & \\
\hline & & & & \\
\hline & & & & \\
\hline & & & & \\
\hline & & & & \\
\hline & & & & \\
\hline & & & & \\
\hline & & & & \\
\hline & & & & \\
\hline & & & & \\
\hline & & & & \\
\hline & & & & \\
\hline & & & & \\
\hline & & & & \\
\hline & & & & \\
\hline
\end{tabular}

Twin Buttes Unique Item log. 
Center for Archaeological Research - UTSA

Twin Buttes Reservoir Survey

PHOTOGRAPHIC LOG

Color [ ] Black/ White [ ] Print [ ] Slide [ ] Film Type _____ Roll \#___-

Camera:

Frame\#

Description

Facing Name Date

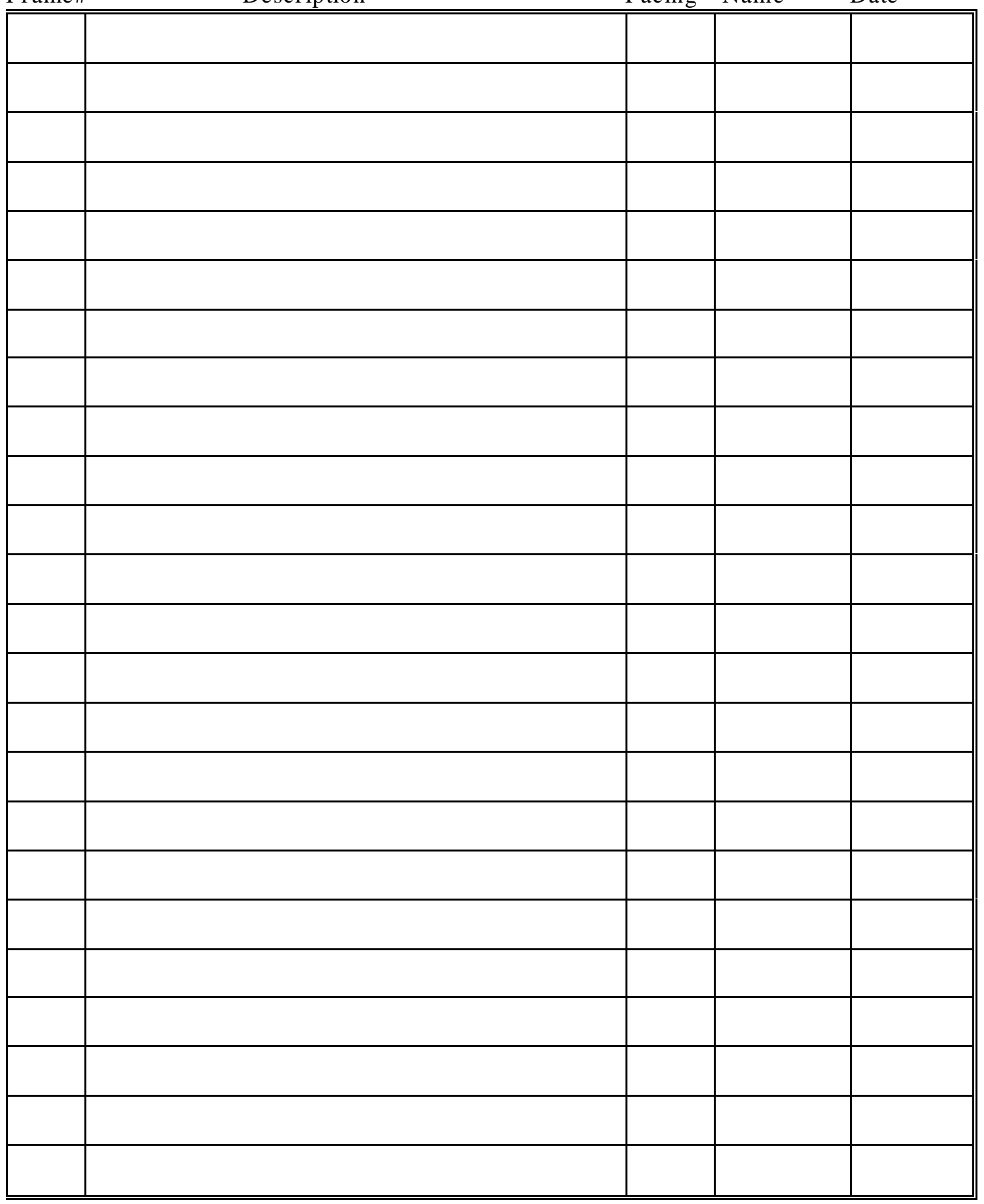

Twin Buttes Photographic log. 


\begin{tabular}{|c|c|c|c|c|c|c|c|c|}
\hline & & & ARCHA & OMAGNE & TIC SAMP & LES & & \\
\hline & & ELD SITE & - TWIN B & UTTES AR & CHAEOLO & GICAL SU & RVEY & \\
\hline & & & & CAR-UTS & & & & \\
\hline & & & & & & Declination & Inclination & \\
\hline Sample \# & Level & Provenience & Northing & Easting & Elevation & (Degrees) & (Degrees) & Intensity \\
\hline & & & & & & & & \\
\hline & & & & & & & & \\
\hline & & & & & & & & \\
\hline & & & & & & & & \\
\hline & & & & & & & & \\
\hline & & & & & & & & \\
\hline & & & & & & & & \\
\hline & & & & & & & & \\
\hline & & & & & & & & \\
\hline & & & & & & & & \\
\hline & & & & & & & & \\
\hline & & & & & & & & \\
\hline & & & & & & & & \\
\hline & & & & & & & & \\
\hline & & & & & & & & \\
\hline & & & & & & & & \\
\hline & & & & & & & & \\
\hline & & & & & & & & \\
\hline & & & & & & & & \\
\hline & & & & & & & & \\
\hline & & & & & & & & \\
\hline & & & & & & & & \\
\hline & & & & & & & & \\
\hline & & & & & & & & \\
\hline & & & & & & & & \\
\hline & & & & & & & & \\
\hline & & & & & & & & \\
\hline & & & & & & & & \\
\hline & & & & & & & & \\
\hline & & & & & & & & \\
\hline & & & & & & & & \\
\hline & & & & & & & & \\
\hline & & & & & & & & \\
\hline & & & & & & & & \\
\hline
\end{tabular}

Twin Buttes Archaeomagnetic Sample log. 


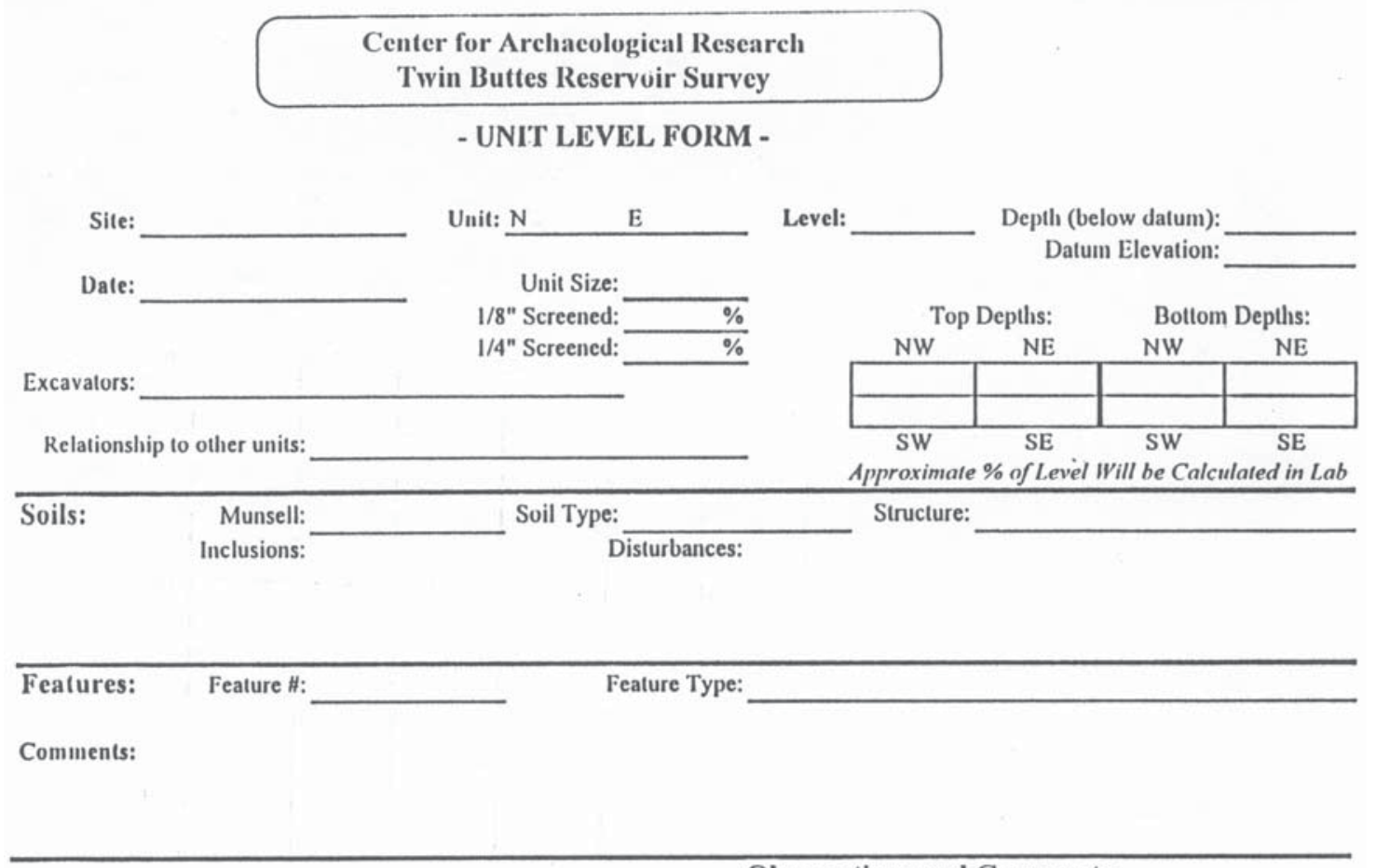

Observations and Comments:

\begin{tabular}{|c|c|c|c|c|c|c|c|}
\hline \multirow[t]{7}{*}{ Bay\# } & Type & $\#$ & Feature \# & \multirow[t]{7}{*}{ Photographs: } & Roll & Frame & \multirow{2}{*}{$\begin{array}{l}\text { Use back of form to sketch } \\
\text { diagnostics as needed. }\end{array}$} \\
\hline & & & & & & & \\
\hline & & & & & & & \multirow{5}{*}{ Supervisor:[ } \\
\hline & & & & & & & \\
\hline & & & & & & & \\
\hline & & & & & & & \\
\hline & & & & & & & \\
\hline
\end{tabular}

Twin Buttes Unit Level Excavation form, page 1. 


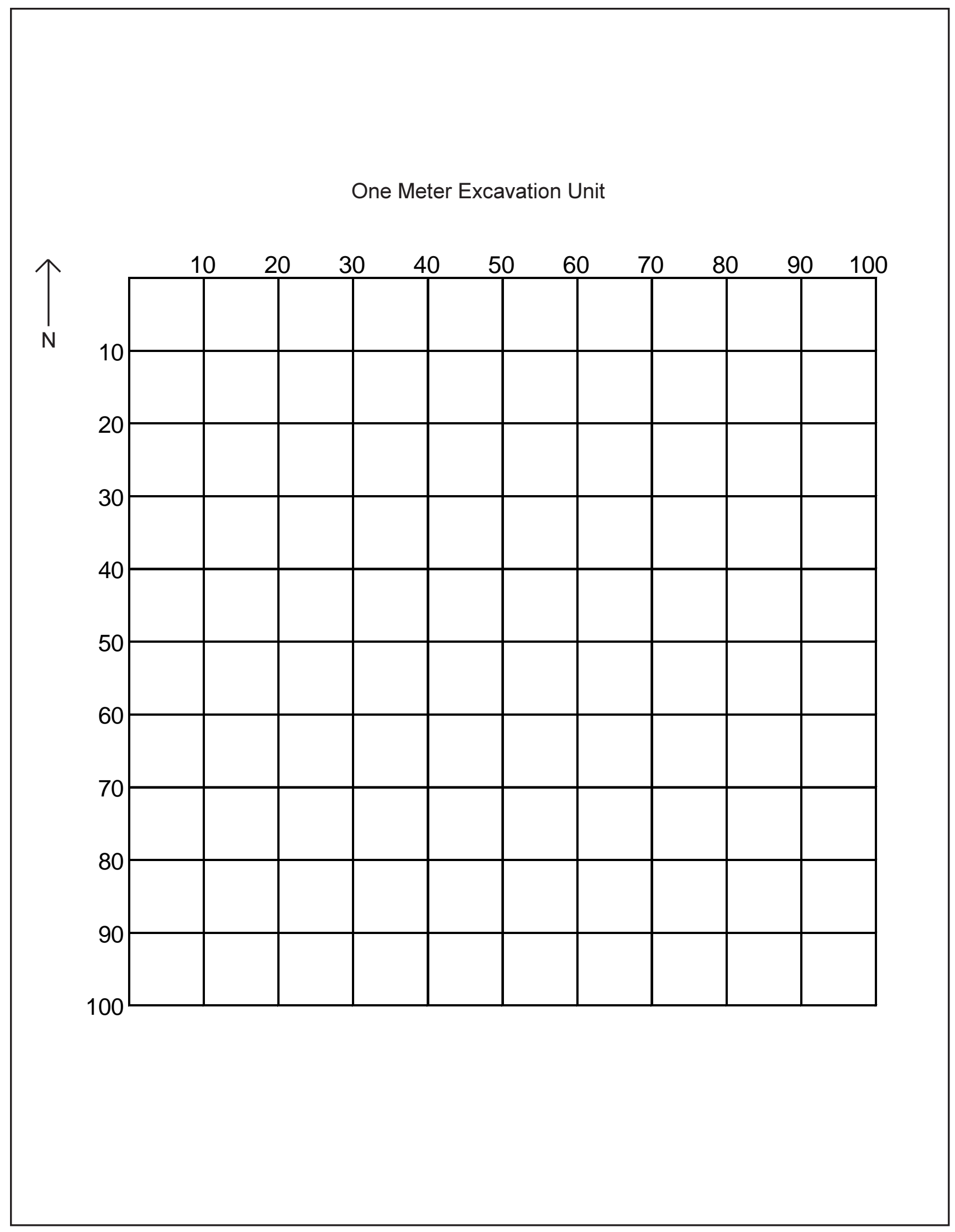

Twin Buttes Unit Level Excavation form, page 2. 
Center for Archaeological Research - UTSA

Twin Buttes Reservoir Survey

COLLECTION BAG LOG

\begin{tabular}{|c|c|c|c|c|c|}
\hline Bag\# & Location & Depth & Contents & Excavator & Date \\
\hline & & & & & \\
\hline & & & & & \\
\hline & & & & & \\
\hline & & & & & \\
\hline & & & & & \\
\hline & & & & & \\
\hline & & & & & \\
\hline & & & & & \\
\hline & & & & & \\
\hline & & & & & \\
\hline & & & & & \\
\hline & & & & & \\
\hline & & & & & \\
\hline & & & & & \\
\hline & & & & & \\
\hline & & & & & \\
\hline & & & & & \\
\hline & & & & & \\
\hline & & & & & \\
\hline & & & & & \\
\hline & & & & & \\
\hline & & & & & \\
\hline & & & & & \\
\hline & & & & & \\
\hline
\end{tabular}

Twin Buttes Collection Bag log. 


\section{CENTER FOR ARCHAEOLOGICAL RESEARCH \\ TWIN BUTTES \\ SURFACE OBSERV ATION AREAS}

Site:

Date:

Rec order:

Page:

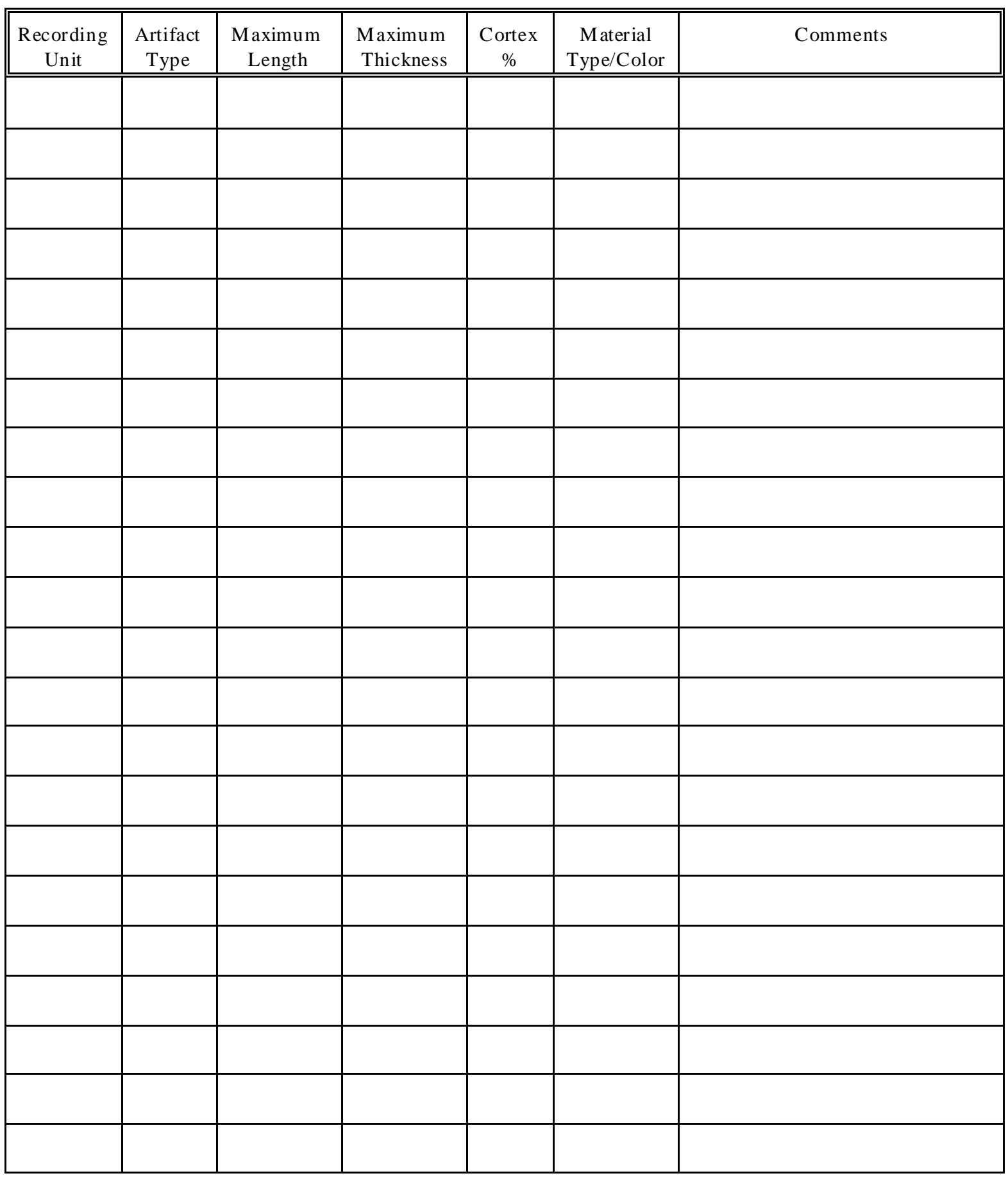

Twin Buttes Surface Observation Area recording form, page 1. 


\section{S URF ACE OBSER VATION D AT A COLLECTION INFORM ATION}

Recording Unit: Refers to the recording unit- area (e.g., A1; A2,....An) or Dog Leash (DL1, DL2...).

Artifact Type: Codes 1 through 7.

1. Flakes. All utilized flakes and/or debris which cannot be classified as a core, tool, or ground stone. Flakes and debris do not necessarily have to have a clearly distinguishable ventral and dorsal surface - pieces often referred to as "chunks" or "angular debris" are of this type.

2. Cores. Cores are defined as any parent block from which flakes have been removed. This category does not, however, include tools. Cores must have at least three negative bulbs and cannot have a positive bulb; if a positive bulb is present in this classification, it is a flake and not a core. "Flake cores" were not a category for the purposes of this analysis.

3. Tested Cobbles. A tested cobble must have had one or two negative bulbs present; no positive bulb.

4. Bifaces. This type includes any item on which both facies have flake scars that cover at least $1 / 3$ of the face and have been removed in a systematic manner. This class includes points, preforms, and blanks. Pieces elsewhere called "bifacial cores" were considered as cores for the purposes of this analysis, and functional use was not considered.

5. Unifaces. This type includes any item on which only one face has systematically removed flakes that cover at least $1 / 3$ of that face. In some cases, unifacial retouch may be accompanied by use and retouch; in this case the piece was still classified as a uniface.

6. Ret ouched/Utilized Flakes. This type includes any item on which flakes have been systematically removed from an edge, but which does no qualify as a biface or uniface.

7. Other. This includes any specimen not covered above such as ground stone, hammer stone, etc. Their identity would be specified in the comments section.

\section{Maximum Length:}

This is the maximum length of an artifact, recorded in $1-\mathrm{cm}$ units ( 0 to $1=1 ; 1$ to $2 \mathrm{~cm}=2 ; 2$ to $3 \mathrm{~cm}=3 \ldots$...tc.).

\section{Maximum Thickness:}

This is the maximum thickness of an artifact, recorded in 1-cm units as shown above for Maximum Length.

Twin Buttes Surface Observation Area recording form, page 2. 


\section{Cortex:}

This is the percentage of cortex on the dorsal surface (including the platform) of all items with the exception of cores, tested cobbles, and the "other" artifact type (see notes below). The following groupings were used:

$$
\begin{array}{lll}
0 \% & =\text { No Cortex } & \text { Code as } 0 \\
1-25 \% & =\text { Cortex } & \text { Code as } 12 \\
26-50 \%=\text { Cortex } & \text { Code as } 38 \\
51-75 \%=\text { Cortex } & \text { Code as } 62 \\
76-99 \%=\text { Cortex } & \text { Code as } 88 \\
100 \%=\text { Cortex } & \text { Code as } 100
\end{array}
$$

On Cores, tested cobbles, and "other" types the following codes were used:
No Cortex
Code as 0
Less than $51 \%$ Cortex
Code as 25
More than $50 \%$ Cortex
Code as 75

\section{Material Ty pe/Color:}

The vast majority of the material was coded as " 1 ", which is either grey, brown, or blue-grey. Codes 2 and 4 are white and/or pale white. Code 3 is either pinkish or red; often heat-treated. Code 9 is any other color.

\section{Comments:}

Any additional information or observations not covered by the above. 



\title{
Appendix L: \\ Twin Buttes Archaeological Project
}

\section{Previous Land Ownership}

\author{
by Tanya Norris, Katie Plum, Preston Stone, and David Nickels
}

This appendix provides a brief synthesis of land ownership prior to 1962 for properties on which historic sites were identified during the Twin Buttes survey. This information was compiled from deed records and maps from the West Texas Collection at Porter Henderson Library, Angelo State University, and the Tom Green County Courthouse Annex, San Angelo, Texas. In addition to land ownership and oil and gas maps, the most current USGS Quadrangle maps (Twin Buttes, Pecan Station, and Knickerbocker) were used to locate more modern structures. In many cases the maps used did not provide complete information, and therefore they are not referenced in the tables.

Map 1 cited in the tables is a Twin Buttes Reservoir Land Acquisition map drawn by the United States Department of the Interior, Bureau of Reclamation, September 27, 1962 (copy on file, CAR-UTSA).

Copies of maps obtained from the West Texas Collection and cited in the tables are:

21945 Ownership Map, Tom Green County.

TW-8 1959 Oil and Gas Map, Tom Green County.

TW-64 1947 Southern Tom Green County Ownership Map.

TW-10 1942 Southern Tom Green County Ownership Map.

31916 Map of Tom Green County.

41894 Map of Tom Green County (Tweedy Collection).

TW-4 1855 Survey Map of Fisher \& Miller's Colony, prepared by G. Schleicher, District Surveyor for Bexar District, San Antonio, 1855.

An additional reference cited most often in Chapter 11 is an extract from a 1923-24 topographical survey map originally prepared by W. H. Griffin, R. W. Buchard, W. J. Lloyd, and H. S. Milsted from which the Twin Buttes Reservoir area was traced over for the reservoir project (copy on file, CAR-UTSA). Handwritten Survey Records are from Survey Record Books in the West Texas Collection at Porter Henderson Library, Angelo State University. Deed Records were obtained from deed books at the Tom Green County Courthouse Annex, San Angelo, Texas.

Tanya Norris, University Archivist, Angelo State University

Katie Plum, Student Assistant, West Texas Collection, Angelo State University

Preston Stone, Student Assistant, West Texas Collection, Angelo State University 


\section{$\underline{41 T G 160}$}

Table L-1. Land ownership of 41TG160 - Survey \#1896

\begin{tabular}{|c|l|l|}
\hline Map \# & Map Reference & Current Owner \\
\hline TW-4 & 1855 Fisher and Miller Colony & In McDonald's District \#11 \\
\hline 4 & 1894 Tom Green County Map & H. Deidrich \\
\hline 3 & 1916 Map of Tom Green County & H. Deidrich \\
\hline TW-64 & 1947 Southern Tom Green County Ownership Map & H. Deidrich \\
\hline TW-8 & 1959 Oil and Gas Map, Tom Green County & H. Deidrich (213 acres) \\
\hline 1 & 1962 Land Ownership Map & H. Deidrich \\
\hline
\end{tabular}

See Figure L-1 for Surveyor's Record.

\section{$\underline{41 T G 248}$}

Table L-2. Land ownership of 41TG248 - Survey \#958 and Survey \#1815

\begin{tabular}{|c|l|l|}
\hline \multicolumn{2}{|c|}{ Survey \#1815 (south side of river) } \\
\hline Map \# & Map Reference & Current Owner \\
\hline TW-4 & 1855 Fisher and Miller Colony & In McDonald's District \#11 \\
\hline 4 & 1894 Tom Green County Map & Samuel W. Woodhouse \\
\hline 3 & 1916 Map of Tom Green County & \\
\hline TW-10 & 1942 Southern Tom Green County Ownership Map & Samuel W. Woodhouse \\
\hline TW-64 & 1947 Southern Tom Green County Ownership Map & Samuel W. Woodhouse \\
\hline TW-8 & 1959 Oil and Gas Map, Tom Green County & Samuel W. Woodhouse (626.5 acres) \\
\hline 1 & 1962 Land Ownership Map & J. Willis Johnson \\
\hline
\end{tabular}

\begin{tabular}{|c|l|l|}
\hline \multicolumn{2}{|c|}{ Survey \#958 (north side of river) } \\
\hline Map \# & Map Reference & Current Owner \\
\hline TW-4 & 1855 Fisher and Miller Colony & In McDonald's District \#11 \\
\hline 4 & 1894 Tom Green County Map & Frans Dorris \\
\hline 3 & 1916 Map of Tom Green County & - \\
\hline TW-10 & 1942 Southern Tom Green County Ownership Map & Frans Dorris (320.9 acres) \\
\hline TW-64 & 1947 Southern Tom Green County Ownership Map & Frans Dorris/J. B. Russell \\
\hline TW-8 & 1959 Oil and Gas Map, Tom Green County & Mrs. Frans Dorris \\
\hline 1 & 1962 Land Ownership Map & Frans Dorris \\
\hline
\end{tabular}

\section{Notes to Survey \#1815 (41TG248):}

Woodhouse, the original grantee, was a doctor who lived in Philadelphia. The property remained in his family until his grandson sold the land in 1940. Samuel Woodhouse, Jr. lived in Maine when he sold the survey to Gordon, Hall and Johnson. All of the men in the Woodhouse family were doctors. 
Deed. T. R. and S. W. Woodhouse, grantee; John Lackey, grantor. March 7, 1881. Woodhouse paid Lackey $\$ 100$ for the land originally granted to Sam Woodhouse that was purchased by Lackey at a tax sale for taxes due in 1877. Date: March 7, 1881. Deed Book D, page 297.

Deed. T. R. Woodhouse by extr. (Philadelphia, Trust, Safe Deposit and Insurance Co.) to Samuel Woodhouse. March 18, 1886. Deed Book Q, page 600.

S.W. Woodhouse dec'd. C/C S. W. Woodhouse last will and testament. Estate given to S.W. Woodhouse Jr. and Matilda Woodhouse, his children. November 14, 1904.

Matilda died and will filed December 29, 1926. Gave Woodhouse Survey to brother, Samuel Jr.

Deed. Samuel Woodhouse Jr. and Catherine, grantor. Kansas City, Mexico and Orient Railway Co., grantee. Easement of land 100 feet wide on each side of the track. Consideration \$200. April 30, 1930. Deed Book 163, page 231 .

Affidavit and Heirship. Affidavit saying S.W. Woodhouse owned survey 1815. The land was granted to his grandfather by letter patent issued by Governor Edward Clark September 1861 recorded 1867. District 11, Toby script No. 449. May 22, 1940. Deed Book 205, page 285.

Deed. Samuel Woodhouse, Jr. sold land to Ruth J. Gordon, Mary B. Hall and J. Willis Johnson. May 14, 1940. Deed Book 205, page 302.

See Figure L-2 for Surveyor's Record.

Notes to Survey \#958 (41TG248):

Deed. Grantor: Franz Dorris; Grantee: Gustavus Schleicher, Date of Instrument: August 14, 1858; Date of Filing: October 17, 1859. B. R. Bexar County Records, page 106.

320 acres in Fisher Miller Colony; Schleicher bought land for $\$ 50$.

Dorris died in Comal County.

No further information discovered. 


\section{$\underline{41 T G 253}$}

Table L-3. Land ownership of 41TG253 - Survey \#811 and Survey \#656

\begin{tabular}{|c|l|l|}
\hline \multicolumn{2}{|c|}{ Survey \#811 (south side of creek) } \\
\hline Map \# & Map Reference & Current Owner \\
\hline TW-4 & 1855 Fisher and Miller Colony & In McDonald's District \#11 \\
\hline 3 & 1916 Map of Tom Green County & - \\
\hline TW-64 & 1947 Southern Tom Green County Ownership Map & J. M. Wagstatt \\
\hline TW-8 & 1959 Oil and Gas Map, Tom Green County & Edward H. Jones (328 acres) \\
\hline 1 & 1962 Land Ownership Map & - \\
\hline
\end{tabular}

\begin{tabular}{|c|l|l|}
\hline \multicolumn{2}{|c|}{ Survey \#656 (north side of creek) } \\
\hline Map \# & Map Reference & Current Owner \\
\hline TW-4 & 1855 Fisher and Miller Colony & Possibly Plot \# 174 in McDonald's District \#11 \\
\hline 3 & 1916 Map of Tom Green County & - \\
\hline TW-64 & 1947 Southern Tom Green County Ownership Map & H. C. White et al. \\
\hline TW-8 & 1959 Oil and Gas Map, Tom Green County & H. C. White (76 acres) \\
\hline 1 & 1962 Land Ownership Map & H. C. White \\
\hline
\end{tabular}

\section{Notes to Survey \#811 (41TG253):}

Patent. State of Texas to Gustavus Schleicher. Filed March 12, 1883. Deed Book F, page 468.

Deed. Gustavus Schleicher to Eleanor Elliott. Sold eight tracks of land on the south bank of Dove Creek and Good Spring Fork of the Concho River; including survey 811. Deed Book F, page 471.

Deed. Eleanor Elliott to Grinnell, Tweedy and Reynolds. Filed March 12, 1883. Deed Book F, page 474.

Warranty Deed, O. W. Matthews and wife to J. M. Wagstatt. Filed April 15, 1927. All of survey 811. Deed Book 128, page 429. (Matthews lived in Tarrant County and Wagstat in Taylor County.)

Release of Vendors Lien between O. W. Matthews and J. M. Wagstatt. August 8 1927. Deed Book 130, page 616.

Nothing in the index that mentioned Wagstatt after 1944. Unable to link Grinnell, Tweedy and Reynolds to Wagstatt.

\section{Notes to Survey \#656 (41TG253):}

No information on H. C. White was found in the indexes up to 1920. A copy of the entry in the Surveyor's Record Book 2 is provided (Figure L-3).

See Figure L-3 for Surveyor's Record. 


\section{$\underline{41 T G 344}$ and $41 T G 459$}

Table L-4. Land ownership of 41TG344 and 41TG459 - Survey \#876

\begin{tabular}{|c|c|c|c|c|c|c|c|}
\hline Survey & Acreage & Date/Map Reference & Grantor & Grantee/Current Owner & Book & Page & Map \# \\
\hline \multirow[t]{3}{*}{876} & & $\begin{array}{l}1855 \text { Fisher and Miller } \\
\text { Colony }\end{array}$ & & $\begin{array}{l}\text { Part of McDonald's District } \\
\# 11\end{array}$ & & & TW-4 \\
\hline & 320 & 18 January 1861 & State of Texas & John O. Meusebach & $\mathrm{D}$ & 492 & \\
\hline & 320 & 1 November 1861 & J.O. Meusebach & Gustave Schleicher & $\mathrm{BR}$ & 435 & \\
\hline \multicolumn{8}{|l|}{$*$} \\
\hline & 320 & 16 July 1881 & Mary A. Maverick & Jacob Fluhr & $\mathrm{D}$ & 497 & \\
\hline & 320 & 19 November 1881 & Jacob Fluhr & John D. Patterson & $\mathrm{D}$ & 635 & \\
\hline & 320 & 2 July 1902 & J.D. Patterson (will) & T.W. \& W.W. Patterson & 26 & 56 & \\
\hline & 320 & 25 April 1906 & T.W. Patterson & George Hagelstein & 26 & 343 & \\
\hline \multicolumn{8}{|l|}{$*$} \\
\hline & 25.5 & 1 December 1913 & George Hagelstein & Frank Jecker & 82 & 249 & \\
\hline & 103.2 & 1 December 1913 & George Hagelstein & Frank Jecker & 82 & 248 & \\
\hline & 25.5 & 5 August 1914 & Frank Jecker, et ux. & S.S. Cummings, et al. & 75 & 189 & \\
\hline & 103.2 & 5 August 1914 & Frank Jecker, et ux. & S.S. Cummings, et al. & 75 & 193 & \\
\hline & & $\begin{array}{l}1916 \text { Map of Tom Green } \\
\text { County }\end{array}$ & & Adam Burkhardt & & & 3 \\
\hline & 50 & 12 November 1917 & J.B. Burns & Sallie Armstrong & 91 & 120 & \\
\hline & 77.3 & 1 December 1919 & S.S. Cummings, et al. & J.W. Cox & 96 & 419 & \\
\hline & 103.2 & 29 November 1922 & Gus Allen, et ux. & J.W. Cox & 106 & 128 & \\
\hline & 103.2 & 3 November 1923 & J.W. Cox & Gus Allen & 108 & 486 & \\
\hline & 50 & 2 April 1924 & Sallie Armstrong & J.W. Cox & 110 & 90 & \\
\hline & 77.3 & 30 October 1935 & J.W. Cox & Rose \& R.F. Gandy & 183 & 198 & \\
\hline \multicolumn{8}{|l|}{ * } \\
\hline & 50 & 30 April 1951 & J.W. Cox & Rose Gandy & & & \\
\hline $\begin{array}{l}\text { Site } \\
41 T G 459\end{array}$ & & $\begin{array}{l}1959 \text { Oil and Gas - } \\
\text { Southern Tom Green } \\
\text { County }\end{array}$ & & Adam Burkhardt & & & TW-8 \\
\hline \multirow[t]{2}{*}{$\begin{array}{l}\text { Site } \\
41 \mathrm{TG} 344\end{array}$} & 127.3 & $\begin{array}{l}1959 \text { Oil and Gas Map, } \\
\text { Tom Green County }\end{array}$ & & J. W. Cox & & & TW-8 \\
\hline & & $\begin{array}{l}1962 \text { Land Ownership } \\
\text { Map }\end{array}$ & & Rose Gandy & & & 1 \\
\hline
\end{tabular}

* Indicates gaps in sequence

Notes to Survey \#816 (41TG344 and 41TG459):

RELEASE OILAND GAS LEASE

HUMBLE OIL AND REFINING CO.

THE STATE OF TEXAS

COUNTY OF HARRIS

TO J.W. COX

KNOW ALL MEN BY THESE PRESENTS:

That Humble Oil and Refining Company does hereby release, remise, and relinquish unto J.W. Cox his successors and assigns, all its rights, titles, and interest in and to that certain oil, and gas mineral lease dated the 16th day of October A.D. 1937 executed by J.W. Cox as Lessor, to Gordon Kenley as Lessee, and of record in Volume 
192 at page 462-65 of the Deed Records of Tom Green County, State of Texas, in so far as said lease covers and includes the following described land situated in Tom Green County, State of Texas...

\section{QUITCLAIM DEED}

STATE OF CALIFORNIA

COUNTY OF LOS ANGELES

KNOW ALL MEN BY THESE PRESENTS, that I, IRENE SPARKS, a widow of the County of Los Angeles, State of California, for and in consideration of the sum of Ten Dollars, cash in hand, paid to me by ROSE GANDY, the receipt of which I hereby acknowledge, have granted, sold, quitclaimed, and conveyed, and by these presents do grant, sell, quitclaim, and convey unto the said ROSE GANDY of Tom Green County, Texas, all of my right, title, and interest in and to all that certain tract of land which is situated in Tom Green County, Texas described as follows:

The West one-half (W. 1/2) of the following described 50 acres of land out of Survey No. 876, Adam Burkhardt, Abstract No. 64, which 50 acres is described by metes and bounds as follows:

Beginning at a stake and stone mound on East line of said Survey No. 876, at the intersection with the North line of the San Angelo and Sherwood Road; Thence North with East line of said Survey No. 876, 649 1/2 varas to a stake from which a mesq. 6" bears S. $93 / 4$ W. 22 varas, a do 8 " bears N. 89 W. 35 varas; Thence West with South line of a 210.9 acre tract sold to $\mathrm{Wm}$. Linbrugger, $4261 / 2$ varas to a stake from which a mesq. 8 " bears N. $3 \frac{3 / 4}{4}$ E. 3 varas, a do 6 " bears S. 38 W. 16 varas; Thence South $4361 / 2$ varas to a stake from which a mesq. 6" bears S. 47 1 1 E. 20 1/2 varas; a do 6" bears N. 70 1/2 W. 11 varas; Thence S. 31 1 2 E. 404 1/2 varas to a stake and stone mound on North side of the San Angelo and Sherwood road, from which a mesq. 5" bears N. 24 3/4 E. 15 1/2 varas; a double mesq. 6 " bears N. 10 1/4 W. $72 \frac{1}{2}$ varas; Thence N. $58 \frac{1}{2} 2$ E. with North line of said road, 252 varas to place of beginning.

TO HAVE AND TO HOLD all of my right, title, and interest in and to the above-described premises, together with all and singular the rights and appurtenances in anywise belonging thereto unto the said Rose Gandy, her heirs and assigns forever.

(Witnessed and signed by George W. Arnold)

Deed Book 433, pages 102-103.

\section{DEED}

I, J.W. COX of the county of Dallas, State of Texas for $\$ 10.00$ and other good and valuable considerations hand paid (in cash) by IRENE SPARKS, have Granted, Sold and Conveyed... unto the said MRS. IRENE SPARKS... all that certain tract or parcel of land lying and being situated in Tom Green County, Texas, described as follows:

(*See deed transferring this land -Survey No. 876- to R. Gandy for description.)

(Witnessed on 30 April 1951 by Pat Tinkle.) 


\section{DEED}

I, J.W. COX of the county of Dallas, State of Texas for $\$ 10.00$ and other good and valuable considerations hand paid (in cash) by ROSE GANDY, have Granted, Sold and Conveyed... unto the said MRS. ROSE GANDY... all that certain tract or parcel of land lying and being situated in Tom Green County, Texas, described as follows:

THE WEST ONE HALF (W. 1/2) of the following described 50 acres of land out of Survey No. 876, Ada [sic], Burkhardt, Abstract No. 64, which 50 acres is described by metes and bounds as follows:

BEGINNING at a stake and stone mound on East line of said Survey No. 876, at the intersection with the North line of the San Angelo and Sherwood Road; THENCE North with East line of said Survey 876, 649 1/2 varas to a stake from which a mesq. 6 " bears S. $93 / 4 \mathrm{~W} .22$ varas, a do 8 " bears N. $89 \mathrm{~W} .35$ varas; THENCE West with South line of a 210.9 acre tract sold to Wm. Linbrugger, $4261 / 2$ varas to a stake from which a mesq. 8 " bears N. $3 \frac{3 / 4}{\text { E. }} 3$ varas, a do 6" bears S. 38 W. 16 varas; THENCE South $436 \frac{1}{2}$ varas to a stake from which a mesq. 6" bears S. $47 \frac{1}{2}$ E. $201 \frac{1}{2}$ varas; a do 6" bears N. $701 \frac{1}{2}$ W. 11 varas; THENCE S. $31 \frac{1}{2}$ E. 404 1 $1 / 2$ varas to a stake and stone mound on North side of the San Angelo and Sherwood road, from which a mesq. 5" bears N. 24 3/4 E. $15 \frac{1}{2}$ varas; a double mesq. $6 "$ bears N. $101 \frac{1}{4}$ W. $72 \frac{1}{2}$ varas; THENCE N. $581 \frac{1}{2}$ E. with North line of said road, 252 varas to place of BEGINNING.

(Witnessed 30 April 1951 by Pat Tinkle)

\section{AGREEMENT OF LEASE}

\section{January 1961:}

EDITH J. MONROE leased to R.F. GANDY 40 7/10 acres out of Survey No. 875, in name of F. Decker; 42 8/10 acres out of Survey No. 876, in name of Adam Burkhardt, for a term of three years, beginning on 1 January 1961, and ending on 31 December 1963, paying $\$ 900.00$ semi-annually in advance on the first day of January and on the first day of July of each year in payments of $\$ 150.00$.

The premises will be used strictly for farming and ranching, and for no other purpose.

This lease shall not interfere with the rights of the Lessor to lease the property for oil or mineral purposes.

Rent shall be paid to Edith J. Monroe, 6526 Fairfield Ave. Berwyn Illinois.

\section{Boundary Line Agreement}

THIS AGREEMENT is made on this, the 12th day of October, 1961, by and between IRENE SPARKS, a widow, on the one hand, and ROSE GANDY, and her husband, R.F. GANDY, on the other hand.

\section{WITNESSETH THAT:}

WHEREAS, by deed from J.W. Cox dated April 30, 1951, and recorded in Volume 309 at page 155 of the Deed Records of Tom Green County, Texas, the west one-half of a certain fifty-acre tact out of Survey No. 876, Adam Burkhardt, Abstract No. 64, was conveyed to the said Rose Gandy which tract is more particularly described in the said deed to which reference is hereby made for all purposes; and, 
WHEREAS, the said J.W. Cox, by deed dated April 30, 1951, recorded in the Deed Records of Tom Green County, Texas in Volume 309 at page 156 conveyed to the said Irene Sparks the east one-half of the said fifty-acre tract above described; and,

WHEREAS, certain questions have arisen concerning the boundary line established between the two tracts by such conveyance; which the parties hereto by these presents do mutually desire to resolve;

NOW, THEREFORE, for and in consideration of the mutual covenance hereinafter set forth, the said IRENE SPARKS, on the one hand, and the said ROSE GANDY and her husband, R.F. GANDY, on the other hand, do hereby covenant and agree that the boundary line which exists between the two tracts hereinabove described is and shall hereafter be described and recognized to be as follows:

"BEGINNING at a point in the North right-of-way boundary of U.S. Highway No. 67 whence the intersection of the East line of the Adam Burkhardt Survey No. 876 and the North right-of-way boundary of U.S. Highway No. 67 bears North fifty eight degrees forty minutes East $\left(\mathrm{N} 58^{\circ} 40^{\prime} \mathrm{E}\right)$, three hundred fifty six and one tenth (356.1) feet; Thence North twenty three degrees eleven minutes West (N23ำ $\left.11^{\prime} \mathrm{W}\right)$, eight hundred nineteen and seven tenths (819.7) feet; Thence North one thousand two hundred twelve and five tenths $(1,212.5)$ feet to the North boundary of said Rose Gandy and Irene Sparks property and the South boundary of the Edith Monroe property."

(Witnesses: Irene Sparks, Rose Gandy, and R.F. Gandy)

Deed Book 433, pages 100-101.

See Figure L-4 for Surveyor's Record.

\section{$\underline{41 T G 412}$}

Table L-5. Land ownership of 41TG412 - Survey \#825

\begin{tabular}{|c|c|c|c|c|c|c|c|}
\hline Survev & Acreage & Date/Map Reference & Grantor & Grantee/Current Owner & Book & Page & Map \# \\
\hline \multirow[t]{2}{*}{825} & 314 & 4 February 1858 & State of Texas & John O. Meusebach & $\mathrm{D}$ & 489 & \\
\hline & 314 & 1 November 1861 & J.O. Meusebach & Gustave Schleicher & $\mathrm{BR}$ & 435 & \\
\hline \multicolumn{8}{|c|}{ (1) } \\
\hline & 314 & 16 July 1881 & Mary A. Maverick & Jacob Fluhr & $\mathrm{D}$ & 497 & \\
\hline & 314 & 19 November 1881 & Jacob Fluhr & John D. Patterson & $\mathrm{D}$ & 635 & \\
\hline & 314 & 25 April 1906 & W.W. \& T.W. Patterson & George Hagelstein & 26 & 343 & \\
\hline & 192 & $\begin{array}{l}1947 \text { Southern Tom Green } \\
\text { County Ownership Map }\end{array}$ & & Wilhelm Leuders & & & TW-64 \\
\hline & & $\begin{array}{l}1959 \text { Oil and Gas - Southern } \\
\text { Tom Green County }\end{array}$ & & Wilhelm Leuders & & & TW-8 \\
\hline & & 1962 Land Ownership Map & & Homer Byrd & & & 1 \\
\hline
\end{tabular}

* Indicates gaps in sequence 


\section{$\underline{41 T G 419}$}

Table L-6. Land ownership of 41TG419 - Survey \#824

\begin{tabular}{|c|c|c|c|c|c|c|c|}
\hline Survey & Acreage & Date/Map Reference & Grantor & Grantee/Current Owner & Book & Page & Map \# \\
\hline \multirow[t]{2}{*}{824} & 246 & 1 November 1861 & J.O. Meusebach & Gustave Schleicher & $\mathrm{BR}$ & 435 & \\
\hline & 246 & 2 April 1866 & Gustave Schleicher & Mexican Gulf R.R. Co. & $\mathrm{C}$ & 762 & \\
\hline \multicolumn{8}{|l|}{$*$} \\
\hline & 246 & 25 April 1906 & T.W. \& W.W. Patterson & George Hagelstein & 26 & 343 & \\
\hline & & $\begin{array}{l}1947 \text { Southern Tom Green } \\
\text { County Ownership Map }\end{array}$ & & L. M. Ratliff et al. & & & TW-64 \\
\hline & & $\begin{array}{l}1959 \text { Oil and Gas - Southern } \\
\text { Tom Green County }\end{array}$ & & W. B. Duncan III & & & TW-8 \\
\hline & & $\begin{array}{l}1959 \text { Oil and Gas - Southern } \\
\text { Tom Green County }\end{array}$ & & Jno. Odstreil & & & TW-8 \\
\hline & & $\begin{array}{l}1959 \text { Oil and Gas - Southern } \\
\text { Tom Green County }\end{array}$ & & Charles C. Motl & & & TW-8 \\
\hline & & $\begin{array}{l}1959 \text { Oil and Gas - Southern } \\
\text { Tom Green County }\end{array}$ & & ERG Blanco & & & TW-8 \\
\hline & & $\begin{array}{l}1959 \text { Oil and Gas - Southern } \\
\text { Tom Green County }\end{array}$ & & Stanford & & & TW-8 \\
\hline & & 1962 Land Ownership Map & & O. D. Harrison & & & 1 \\
\hline
\end{tabular}

* Indicates gaps in sequence

See Figure L-5 for Surveyor's Record.

\section{$\underline{41 T G 443}$ and $41 T G 445$}

Table L-7. Land ownership of 41TG443 and 41TG445 - Survey \#104

\begin{tabular}{|c|l|l|}
\hline Map \# & Map Reference & Current Owner \\
\hline TW-4 & 1855 Fisher and Miller Colony & Plot \# 174 or 175 in McDonald's District \#11 \\
\hline 3 & 1916 Map of Tom Green County & Washington County School \\
\hline TW-64 & 1947 Southern Tom Green County Ownership Map & Washington County School \\
\hline TW-8 & 1959 Oil and Gas - Southern Tom Green County & Washington County School \\
\hline TW-8 & 1959 Oil and Gas - Southern Tom Green County & J. R. Medlock - 640 acres - 1933? \\
\hline 1 & 1962 Land Ownership Map & Washington County School \\
\hline
\end{tabular}

Notes to Survey \#104 (41TG443):

\section{Land Patent}

Deed Book B, page 245.

August 22, 1877

Filed September 18, 1877. 
Land Patent awarded by P. H. Bell, Governor of Texas to the school Commissioners of Washington County. One league of land in Bexar County known as survey 104 in Sec. No. 14 on the west bank of the south fork of the Concho River. The NE corner of survey 102 from which a pecan bears E. ...

\section{Lease}

Deed Book S, page 422.

Date of instrument: July 7, 1886.

Date of filing: October 16, 1886.

In May 1883 C. B. Metcalfe leased four leagues of Washington Co. School Land at 13 1/2 cents per acre per annum for the term of five years beginning July 1, 1888. Metcalfe agreed to pay the sum of $\$ 239.12$ annually in advance. Metcalfe canceled the agreement and left a lease for the remaining term of 2 years, July 11886 - July 11888.

Beginning July 1886 Washington County leased the land to Seaton Keith for $131 / 2$ cents per acre. This leased land was on both sides of the Concho River. Seaton Keith agreed to use all necessary means and labor to protect and care for the timber on the said land. Lease expired July 1, 1888.

Searched indexes through 1970 and found no other Washington County entries that referred to survey 104.

See Figure L-6 for Surveyor's Record.

\section{$\underline{41 T G 450}$}

Table L-8. Land ownership of 41TG450 - Survey \#653

\begin{tabular}{|c|l|l|}
\hline Map \# & Map Reference & Current Owner \\
\hline TW-4 & 1855 Fisher and Miller Colony & Possibly Plot \# 174 in McDonald's District \#11 \\
\hline 3 & 1916 Map of Tom Green County & James Davis \\
\hline TW-64 & 1947 Southern Tom Green County Ownership Map & James Davis \\
\hline TW-8 & 1959 Oil and Gas - Southern Tom Green County & James Davis \\
\hline 1 & 1962 Land Ownership Map & E. Hobbs \\
\hline
\end{tabular}

See Figure L-7 for Surveyor's Record.

\section{$\underline{41 T G 452}$}

Table L-9. Land ownership of 41TG452 - Survey \#654

\begin{tabular}{|c|l|l|}
\hline Map \# & Map Reference & Current Owner \\
\hline TW-4 & 1855 Fisher and Miller Colony & Possibly Plot \# 174 in McDonald's District \#11 \\
\hline 3 & 1916 Map of Tom Green County & Probandt and Raphael \\
\hline TW-64 & 1947 Southern Tom Green County Ownership Map & Probandt and Raphael \\
\hline TW-8 & 1959 Oil and Gas - Southern Tom Green County & Joe Sawyer \\
\hline 1 & 1962 Land Ownership Map & Joe Sawyer \\
\hline
\end{tabular}

See Figure L-8 for Surveyor's Record. 


\section{$\underline{41 T G 458}$}

Table L-10. Land ownership of 41TG458 - Survey \#875

\begin{tabular}{|c|c|c|c|c|c|c|c|}
\hline Survey & Acreage & Date/Map Reference & Grantor & Grantee/Current Owner & Book & Page & Map \# \\
\hline \multirow[t]{3}{*}{875} & & 1855 Fisher and Miller Colony & & $\begin{array}{l}\text { Possibly Plot \# } 174 \text { in } \\
\text { McDonald's District \#11 }\end{array}$ & & & TW-4 \\
\hline & 320 & 1 November 1861 & J.O. Meusebach & Gustave Schleicher & $\mathrm{BR}$ & 435 & \\
\hline & 320 & 1 July 1873 & State of Texas & Samuel A. Maverick & $\mathrm{D}$ & 491 & \\
\hline \multirow[t]{5}{*}{ * } & & & & & & & \\
\hline & 320 & 16 July 1881 & Mary A. Maverick & Jacob Fluhr & $\mathrm{D}$ & 497 & \\
\hline & 320 & 19 November 1881 & Jacob Fluhr & John D. Patterson & $\mathrm{D}$ & 635 & \\
\hline & 320 & 2 July 1902 & J.D. Patterson (will) & T.W. \& W.W. Patterson & 26 & 56 & \\
\hline & 320 & 25 April 1906 & T.W. Patterson & George Hagelstein & 26 & 343 & \\
\hline \multicolumn{8}{|c|}{ ( } \\
\hline & 43.2 & 19 February 1913 & P.J. Baron (will-J.D.P) & George Hagelstein & 77 & 338 & \\
\hline & 46.6 & 1 December 1913 & George Hagelstein & Adel Jecker & 74 & 561 & \\
\hline & 163.3 & 1 December 1913 & George Hagelstein & Frank Jecker & 82 & 249 & \\
\hline & 101.4 & 1 December 1913 & George Hagelstein & Frank Jecker & 82 & 248 & \\
\hline & 101.4 & 5 August 1914 & Frank Jecker, et ux. & S.S. Cummings, et al. & 75 & 189 & \\
\hline & 163.3 & 5 August 1914 & Frank Jecker, et ux. & S.S. Cummings, et al. & 75 & 193 & \\
\hline & 46.6 & 5 August 1914 & Adel Jecker & S.S. Cummings, et al. & 75 & 191 & \\
\hline & & $\begin{array}{l}1916 \text { Map of Tom Green } \\
\text { County }\end{array}$ & & Illegible & & & 3 \\
\hline & 163.3 & 1 December 1919 & S.S. Cummings, et al. & J.W. Cox & 96 & 419 & \\
\hline & 102.9 & 29 November 1922 & Gus Allen, et ux. & J.W. Cox & 106 & 128 & \\
\hline & 102.9 & 3 November 1923 & J.W. Cox & Gus Allen & 108 & 486 & \\
\hline & 163.3 & 30 October 1935 & J.W. Cox & Rose \& R.F. Gandy & 183 & 198 & \\
\hline & & $\begin{array}{l}1947 \text { Southern Tom Green } \\
\text { County Ownership Map }\end{array}$ & & Illegible & & & TW-64 \\
\hline & & $\begin{array}{l}1959 \text { Oil and Gas - Southern } \\
\text { Tom Green County }\end{array}$ & & Rose Gandy & & & TW-8 \\
\hline & & 1962 Land Ownership Map & & Thomas A. Childress & & & 1 \\
\hline
\end{tabular}

* Indicates gaps in sequence

See Figure L-9 for Surveyor's Record. 


\section{$\underline{41 T G 501}$}

Table L-11. Land ownership of 41TG501 - Survey \#817

\begin{tabular}{|c|c|c|c|c|c|c|}
\hline Acreage & Date/Map Reference & Grantor & Grantee/Current Owner & Book & Page & Map \# \\
\hline & 1855 Fisher and Miller Colony & & Part of McDonald's District \#11 & & & TW-4 \\
\hline \multirow[t]{6}{*}{160} & 1856 & Fisher and Miller & Phillip Huppman & $\begin{array}{l}\text { Survey Record } \\
\text { Book } 2\end{array}$ & 289 & \\
\hline & 1894 Map of Tom Green County & & P. Huppmann (Patent \#5138) & & & 4 \\
\hline & 1916 Map of Tom Green County & & illegible & & & 3 \\
\hline & 1942 Map of Tom Green County & & P. Huppmann (Patent \#5138) & & & \\
\hline & $\begin{array}{l}1947 \text { Southern Tom Green County } \\
\text { Ownership Map }\end{array}$ & & P. Huppmann (J. D. Suggs estate) & & & TW-64 \\
\hline & $\begin{array}{l}1959 \text { Oil and Gas - Southern Tom } \\
\text { Green County }\end{array}$ & & P. Huppmann & & & TW-8 \\
\hline 155.8 & 1959 Map of Tom Green County & & O. McGowan et al. & & & \\
\hline \multirow[t]{2}{*}{154} & 1962 Land Ownership Map & & O. McGowan et al. & & & 1 \\
\hline & 1965 Map of Tom Green County & & C. M. McGowan et al. & & & \\
\hline
\end{tabular}

See Figure L-10 for Surveyor's Record.

\section{$\underline{41 T G 504}$}

Table L-12. Land ownership of 41TG504 - Survey \#888

\begin{tabular}{|c|c|c|c|c|c|c|}
\hline Acreage & Date/Map Reference & Grantor & Grantee/Current Owner & Book & Page & Map \# \\
\hline & 1855 Fisher and Miller Colony & & Part of McDonald's District \#11 & & & TW-4 \\
\hline \multirow[t]{6}{*}{160} & & Fisher and Miller & Heirs of Joseph Jung & $\begin{array}{l}\text { Survey Record } \\
\text { Book } 2\end{array}$ & 210 & \\
\hline & 1894 Tom Green County Map & & Joseph Jung & & & 4 \\
\hline & 1916 Map of Tom Green County & & Illegible & & & 3 \\
\hline & $\begin{array}{l}1942 \text { Southern Tom Green County } \\
\text { Ownership Map }\end{array}$ & & Joseph Jung & & & TW-10 \\
\hline & $\begin{array}{l}1947 \text { Southern Tom Green County } \\
\text { Ownership Map }\end{array}$ & & Joe Jung & & & TW-64 \\
\hline & $\begin{array}{l}1959 \text { Oil and Gas - Southern Tom } \\
\text { Green County }\end{array}$ & & Joe Jung & & & TW-8 \\
\hline 160 & 1959 Map of Tom Green County & & Edward H. Jones & & & \\
\hline 160 & 1962 Land Ownership Map & & Joe Jung & & & 1 \\
\hline & 1965 Map of Tom Green County & & E. H. Jones & & & \\
\hline
\end{tabular}

See Figure L-11 for Surveyor's Record. 


\section{$\underline{41 T G 513}$}

Table L-13. Land ownership of 41TG513 - Survey \#102

\begin{tabular}{|c|c|c|c|c|c|c|}
\hline Acreage & Date/Map Reference & Grantor & Grantee/Current Owner & Book & Page & Map \# \\
\hline \multirow[t]{2}{*}{$\begin{array}{l}\text { half league } \\
\text { or } 1 / 2 \text { labor }\end{array}$} & 1849 & $\begin{array}{l}\text { Board of Land Comm. } \\
\text { County of Red River }\end{array}$ & David Lloyd & $\begin{array}{l}\text { Survey Record } \\
\text { Book } 1\end{array}$ & 480 & \\
\hline & 1855 Fisher and Miller Colony & & $\begin{array}{l}\text { Part of McDonald's District } \\
\# 11\end{array}$ & & & TW-4 \\
\hline \multirow[t]{3}{*}{640} & 1886 & C. A. Ragsdale & J. N. Upton & & & \\
\hline & 1887 & J. N. Upton & J. W. Johnson & & & \\
\hline & 1894 Tom Green County Map & & David Lloyd & & & 4 \\
\hline Plat I-840 & 1942 Tom Green County Map & & David Lloyd & & & \\
\hline Plat I-840 & $\begin{array}{l}1947 \text { Southern Tom Green } \\
\text { County Ownership Map }\end{array}$ & & David Lloyd & & & TW-64 \\
\hline 1,400 & $\begin{array}{l}1959 \text { Oil and Gas - Southern } \\
\text { Tom Green County }\end{array}$ & & David Lloyd & & & TW-8 \\
\hline 640 & 1959 Tom Green County Map & & $\begin{array}{l}\text { Mrs. J.T. Jenson/ J.W. } \\
\text { Shepperson }\end{array}$ & & & \\
\hline 1,318 & 1959 Tom Green County Map & & Gene Whitehead & & & \\
\hline 376 & $\begin{array}{l}\text { 1959-65 Tom Green County } \\
\text { Maps }\end{array}$ & & W.C. Hoelscher & & & \\
\hline 568.8 & 1962 Land Ownership Map & & G. D. Morgan & & & 1 \\
\hline 1318 & 1965 Tom Green County Map & & Alfredo D. Braden et al. & & & \\
\hline \multirow[t]{2}{*}{$55.4(?)$} & 1965 Tom Green County Map & & A.A. Hoelscher & & & \\
\hline & 1965 Tom Green County Map & & $\begin{array}{l}\text { Ann W. McGowan/ J.W. } \\
\text { Shepperson }\end{array}$ & & & \\
\hline
\end{tabular}

\section{Notes to Survey \#102 (41TG513):}

Field Notes of a survey of a Half League of a half labor land made for David Lloyd it being part of a quantity of land to which he is entitled by virtue of Certificate issued be the Board of Land for the county of Red River on the 5 th day of July 1838 . No. 655.

Said survey is 102 in Section 14 situated on the South Fork of the Concho river about 58 miles NW of the Old San Saba Fort being at a stake on the West bank of Concho River.

See Figure L-12 for Surveyor's Record. 


\section{TG516}

Table L-14. Land ownership of 41TG516 - Survey \#814

\begin{tabular}{|c|c|c|c|c|c|c|c|}
\hline Survey & Acreage & Date/Map Reference & Grantor & Grantee/Current Owner & Book & Page & Map \# \\
\hline 814 & & 1855 Fisher and Miller Colony & & $\begin{array}{l}\text { Probably Plot \#166 in } \\
\text { McDonald's District \#11 }\end{array}$ & & & TW-4 \\
\hline & 561 & 10 July 1882 & Mary A. Maverick & T.J. Wiley & $\mathrm{F}$ & 18 & \\
\hline & 561 & 15 February 1884 & T.J. Wiley & John R. Nasworthy & $\mathrm{J}$ & 41 & \\
\hline & 561 & 20 October 1886 & John R. Nasworthy & William Lackey & $\mathrm{S}$ & 441 & \\
\hline & 561 & 15 October 1888 & William Lackey, et ux. & J.S. Fowlkes & $\mathrm{Y}$ & 44 & \\
\hline & & 1894 Tom Green County Map & & Mrs. Heinr. Roth & & & 4 \\
\hline & & 1916 Map of Tom Green County & & Charles Mott & & & 3 \\
\hline & 110 & 6 October 1929 & R.D. Loika & J.M. Wagstaff & 157 & 140 & \\
\hline & 174 & 9 October 1929 & Wencel Motl, et ux. & J.M. Wagstaff & 157 & 139 & \\
\hline & 100 & 24 December 1929 & Frank Motl, et ux. & J.M. Wagstaff & 157 & 517 & \\
\hline & & $\begin{array}{l}1942 \text { Southern Tom Green County } \\
\text { Ownership Map }\end{array}$ & & H. Roth & & & TW-10 \\
\hline & & $\begin{array}{l}1945 \text { Tom Green County Land } \\
\text { Ownership Map }\end{array}$ & & Heirs of H. Roth & & & 2 \\
\hline & & $\begin{array}{l}1947 \text { Southern Tom Green County } \\
\text { Ownership Map }\end{array}$ & & R. Roth & & & TW-64 \\
\hline & 94 & $\begin{array}{l}1959 \text { Oil and Gas - Southern Tom } \\
\text { Green County }\end{array}$ & & D. E. Switzer & & & TW-8 \\
\hline & & 1962 Land Ownership Map & & Dawse E. Switzer & & & 1 \\
\hline
\end{tabular}

See Figure L-13 for Surveyor's Record. 


\section{TG520, 41TG521, and 41TG523}

Table L-15. Land ownership of 41TG520, 41TG521, 41TG523 - Survey \#647

\begin{tabular}{|c|c|c|c|c|c|c|}
\hline Acreage & Date/Map Reference & Grantor & Grantee/Current Owner & Book & Page & Map \# \\
\hline & 1855 Fisher and Miller Colony & & $\begin{array}{l}\text { Plot \#166 in McDonald's } \\
\text { District \#11 }\end{array}$ & & & TW-4 \\
\hline & 1856 & Fisher and Miller & \begin{tabular}{|l} 
Heirs of Phillip \\
Kurzenocker
\end{tabular} & $\begin{array}{l}\text { Survey Record } \\
\text { Book } 2\end{array}$ & 340 & \\
\hline & 1894 Tom Green County Map & & Phil Kurzenocker & & & 4 \\
\hline & 1916 Tom Green County Map & & Phil Kurzenocker & & & 3 \\
\hline 608 & 1922 & A. Lee/ E. \& T. J. Caldwell & R. C. DeLong & 105258 & & \\
\hline $150 \quad 1 / 10$ & 1926 & R. C. DeLong & H. A. Montgomery & 124551 & & \\
\hline 75 & 1927 & H. A. Montgomery & E. V. Morris & 13634 & & \\
\hline $150 \quad 1 / 10$ & 1929 & H. A. Montgomery & J. M. Wagstaff & 157340 & & \\
\hline 75 & 1929 & E. V. Morris & J. M. Wagstaff & 157339 & & \\
\hline 178.62 & 1929 & R. C. DeLong & G. W. Fry & 153210 & & \\
\hline 178.62 & 1929 & G. W. Fry & J. M. Wagstaff & 192174 & & \\
\hline 68.5 & 1929 & & H. C. Ragsdale & 153210 & & \\
\hline 109.6 & 1929 & & Frank Daugherty & 153210 & & \\
\hline 73.939 & 1940 & West Texas Utilities & J. F. Key & 22760 & & \\
\hline 94.594 & 1941 & West Texas Utilities & J. F. Key & 208610 & & \\
\hline \multirow[t]{6}{*}{1332.745} & 1941 & West Texas Utilities & J. F. Key & 22761 & & \\
\hline & $\begin{array}{l}1942 \text { Southern Tom Green } \\
\text { County Ownership Map }\end{array}$ & & Heirs of P. Kurzenocker & & & TW-10 \\
\hline & $\begin{array}{l}1945 \text { Tom Green County Land } \\
\text { Ownership Map }\end{array}$ & & Heirs of P. Kurzenocker & & & 2 \\
\hline & $\begin{array}{l}1947 \text { Southern Tom Green } \\
\text { County Ownership Map } \\
\end{array}$ & & P. Kurzenocker & & & TW-64 \\
\hline & $\begin{array}{l}1959 \text { Oil and Gas - Southern } \\
\text { Tom Green County }\end{array}$ & & J. F. Key (132.7 acres) & & & TW-8 \\
\hline & 1962 Land Ownership Map & & J. F. Key & & & 1 \\
\hline
\end{tabular}

See Figure L-14 for Surveyor's Record. 


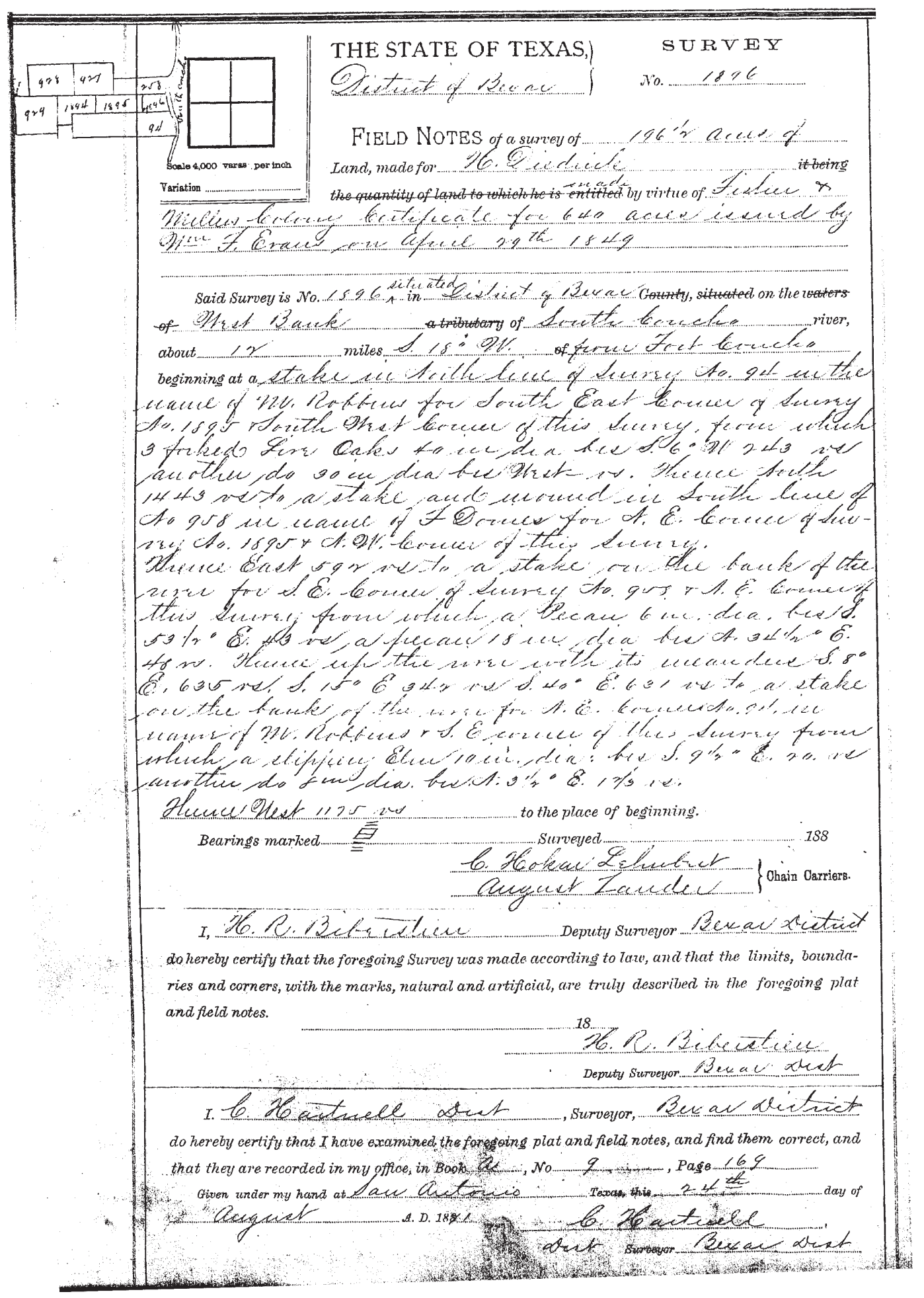

Figure L-1. Survey \#1896 (41TG160), Surveyor's Record, Book 1, Tom Green County. West Texas Collection, Angelo State University. 


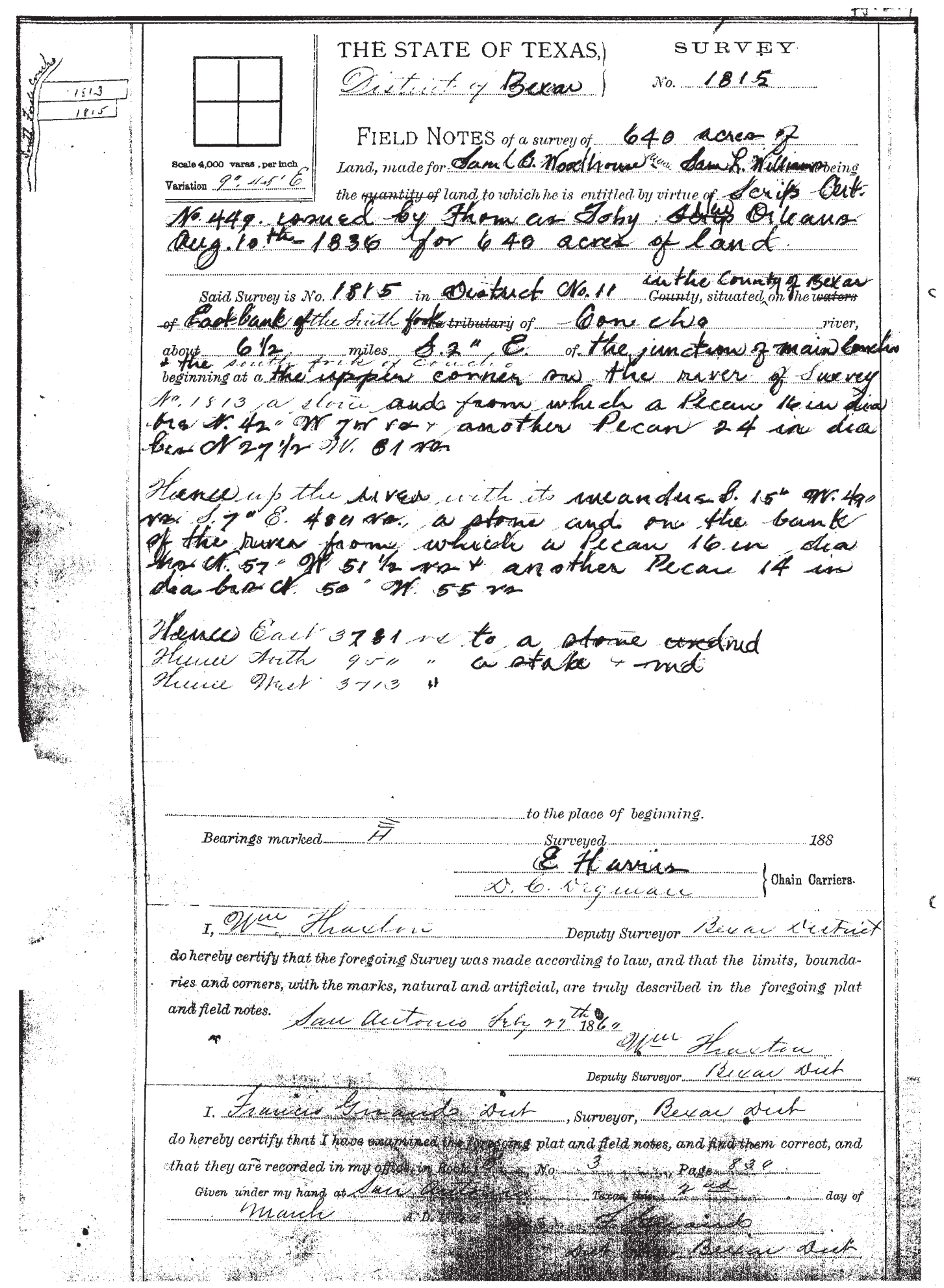

Figure L-2. Survey \#1815 (41TG248), Surveyor's Record, Book 1, page 214, Tom Green County. West Texas Collection, Angelo State University. 


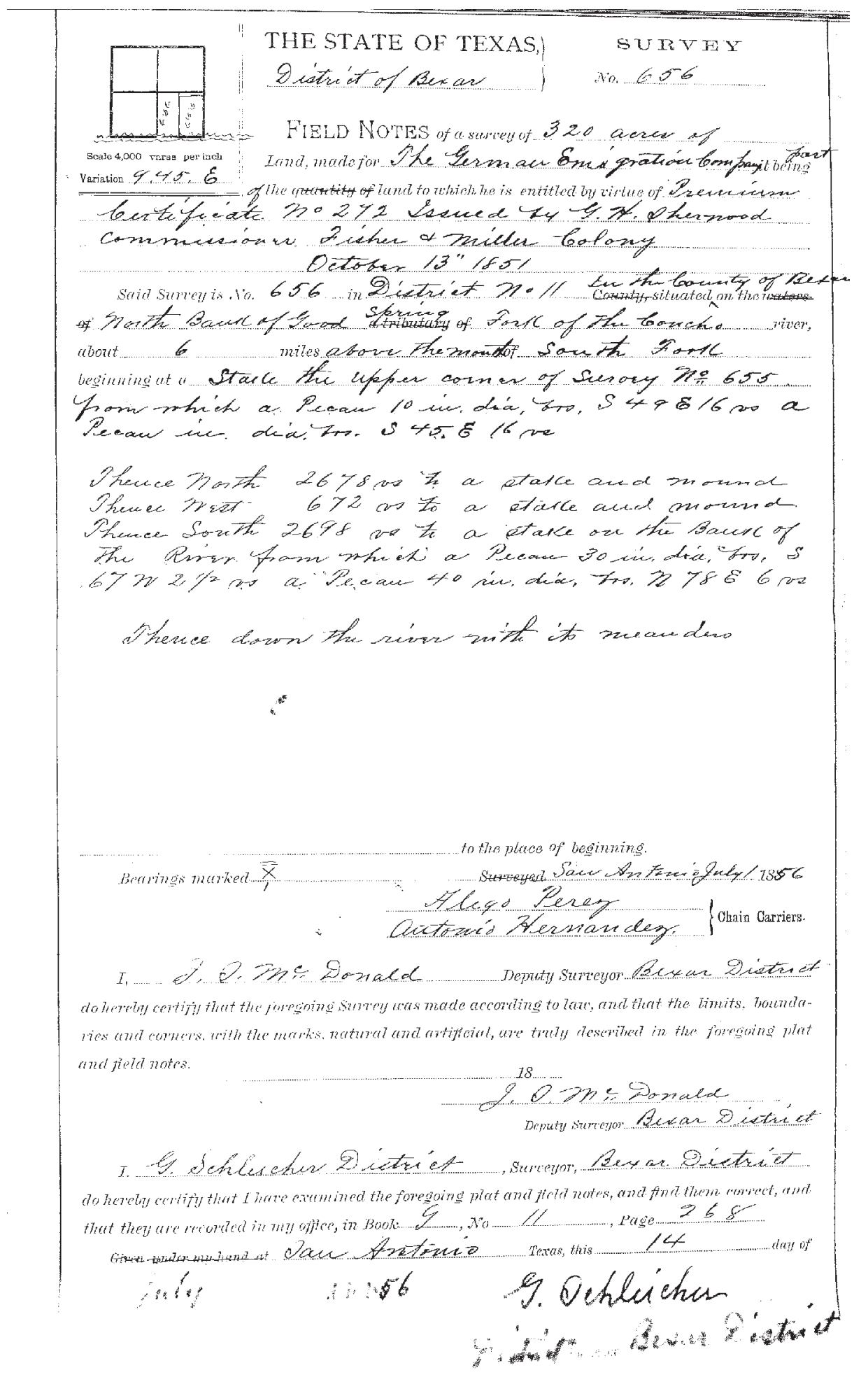

Figure L-3. Survey \#656 (41TG253), Surveyor's Record, Book 2, page 9. West Texas Collection, Angelo State University. 


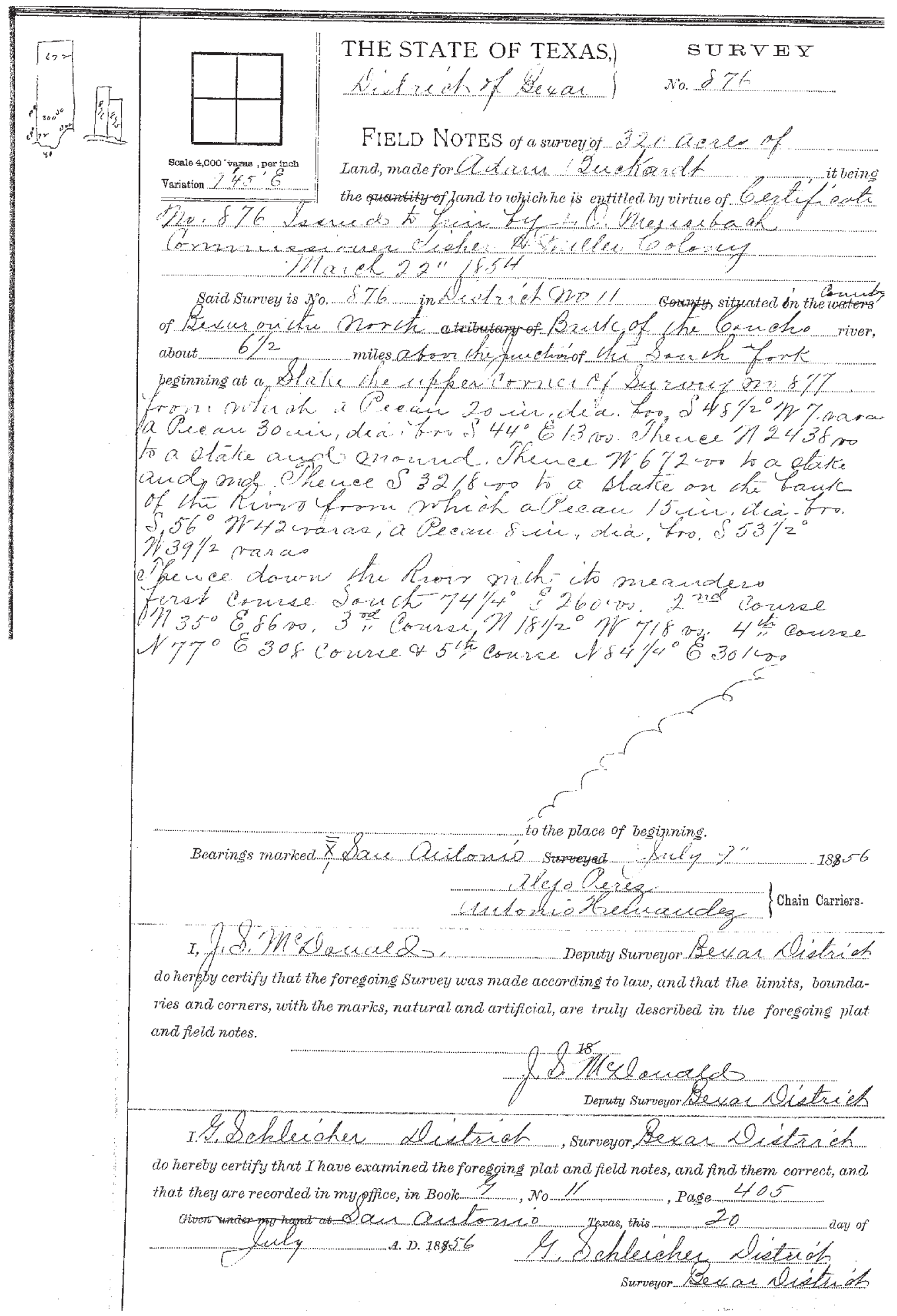

Figure L-4. Survey \#876 (41TG344 and 41TG459), Surveyor's Record, Book 2, page 315. West Texas Collection, Angelo State University. 


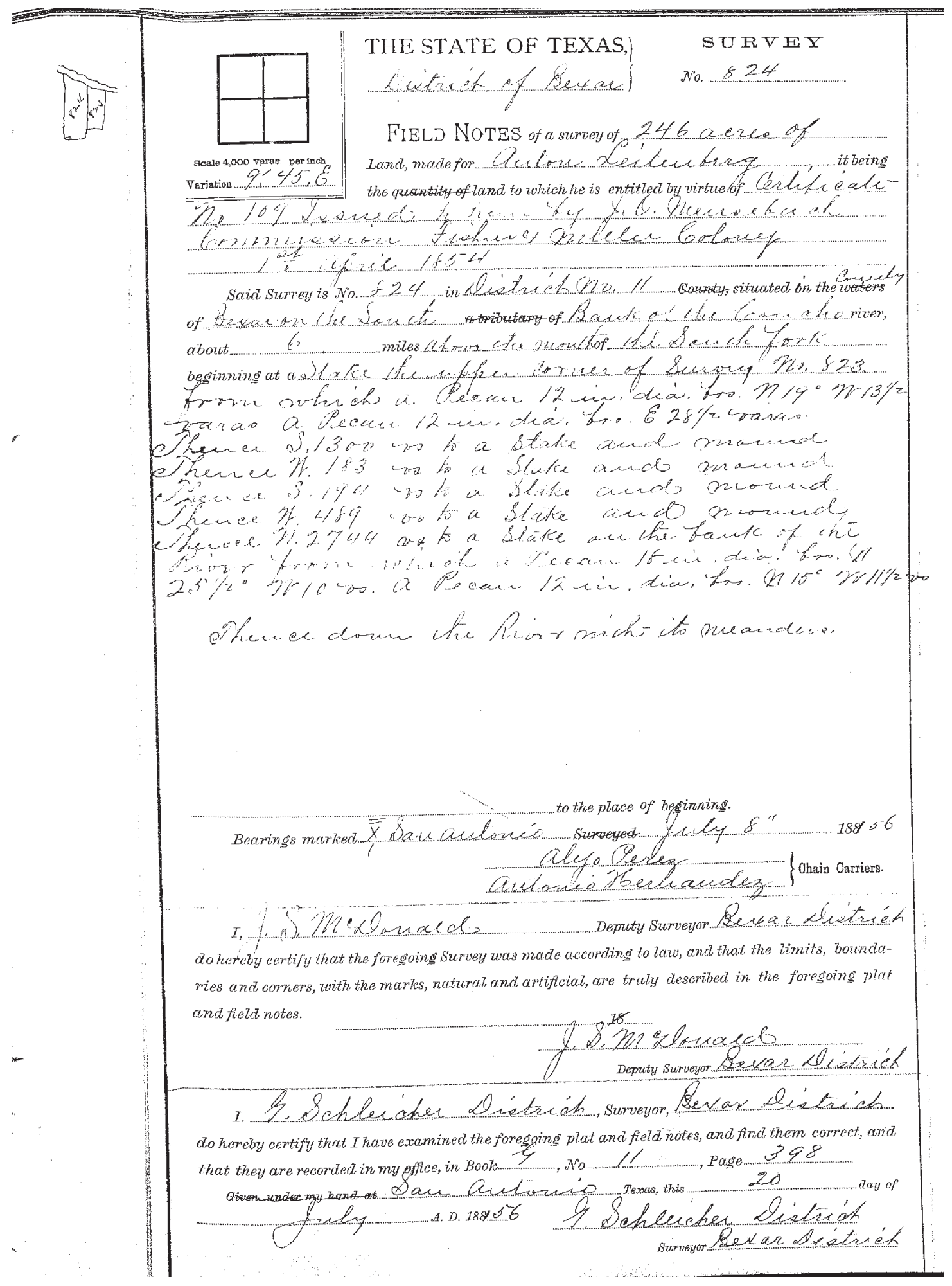

Figure L-5. Survey \#824 (41TG419), Surveyor's Record, Book 2, page 311. West Texas Collection, Angelo State University. 


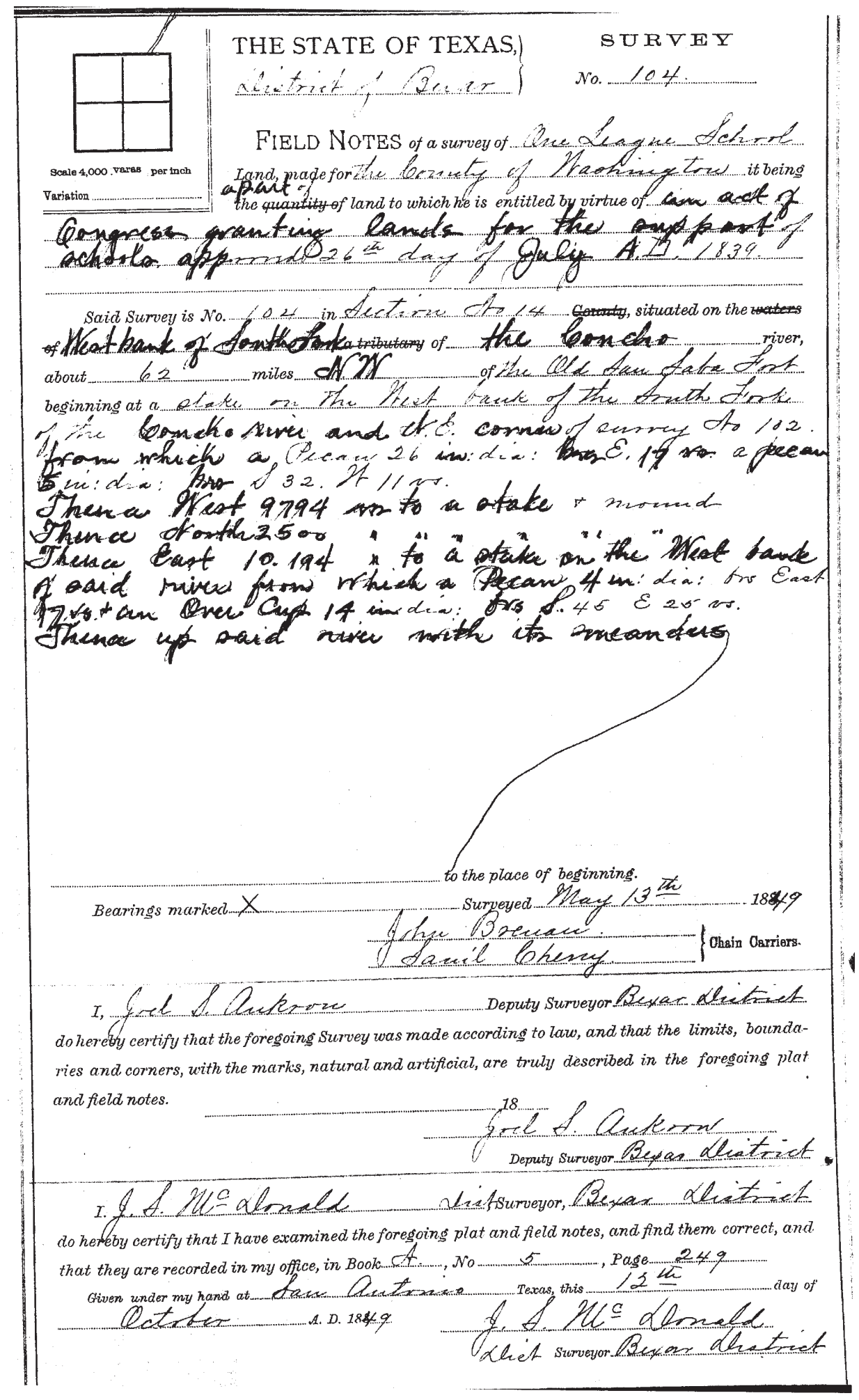

Figure L-6. Survey \#104 (41TG445), Surveyor's Record, Book 1, page 479, Tom Green County. West Texas Collection, Angelo State University. 


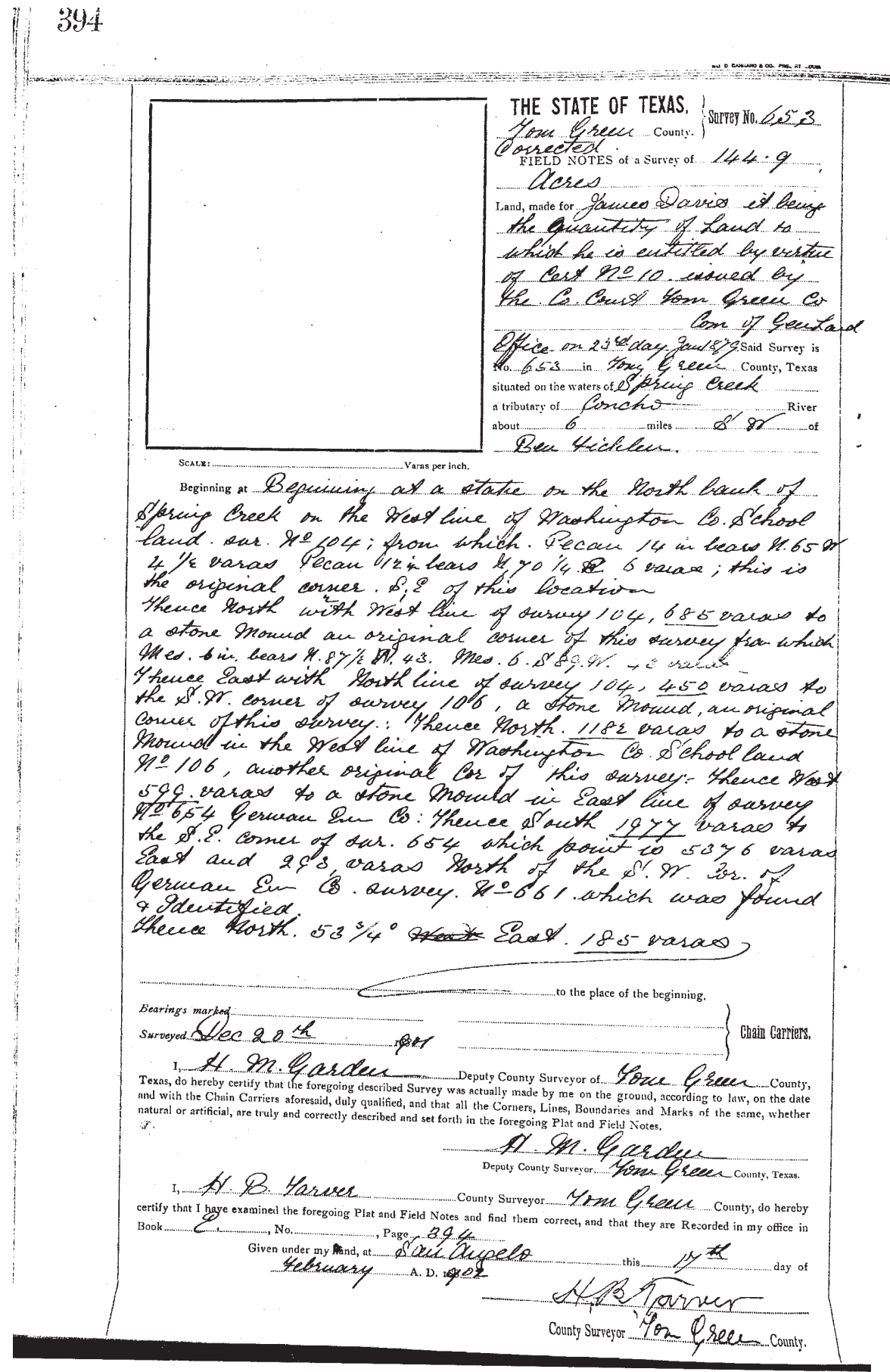

Figure L-7. Survey \#653 (41TG450), Surveyor's Record, School Land E, page 394. West Texas Collection, Angelo State University. 


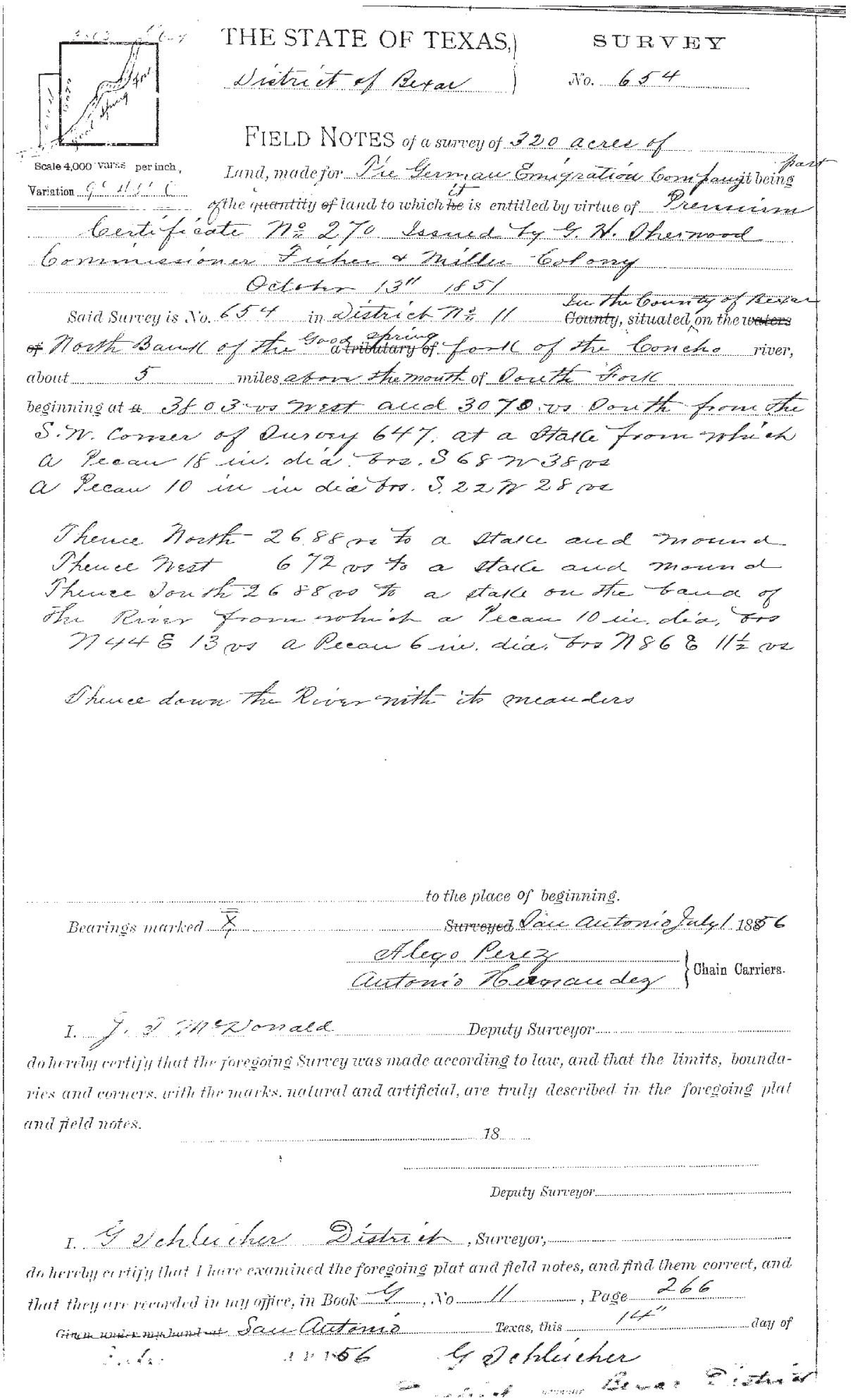

Figure L-8. Survey \#654 (41TG452), Surveyor's Record, Book 2, page 7. West Texas Collection, Angelo State University. 


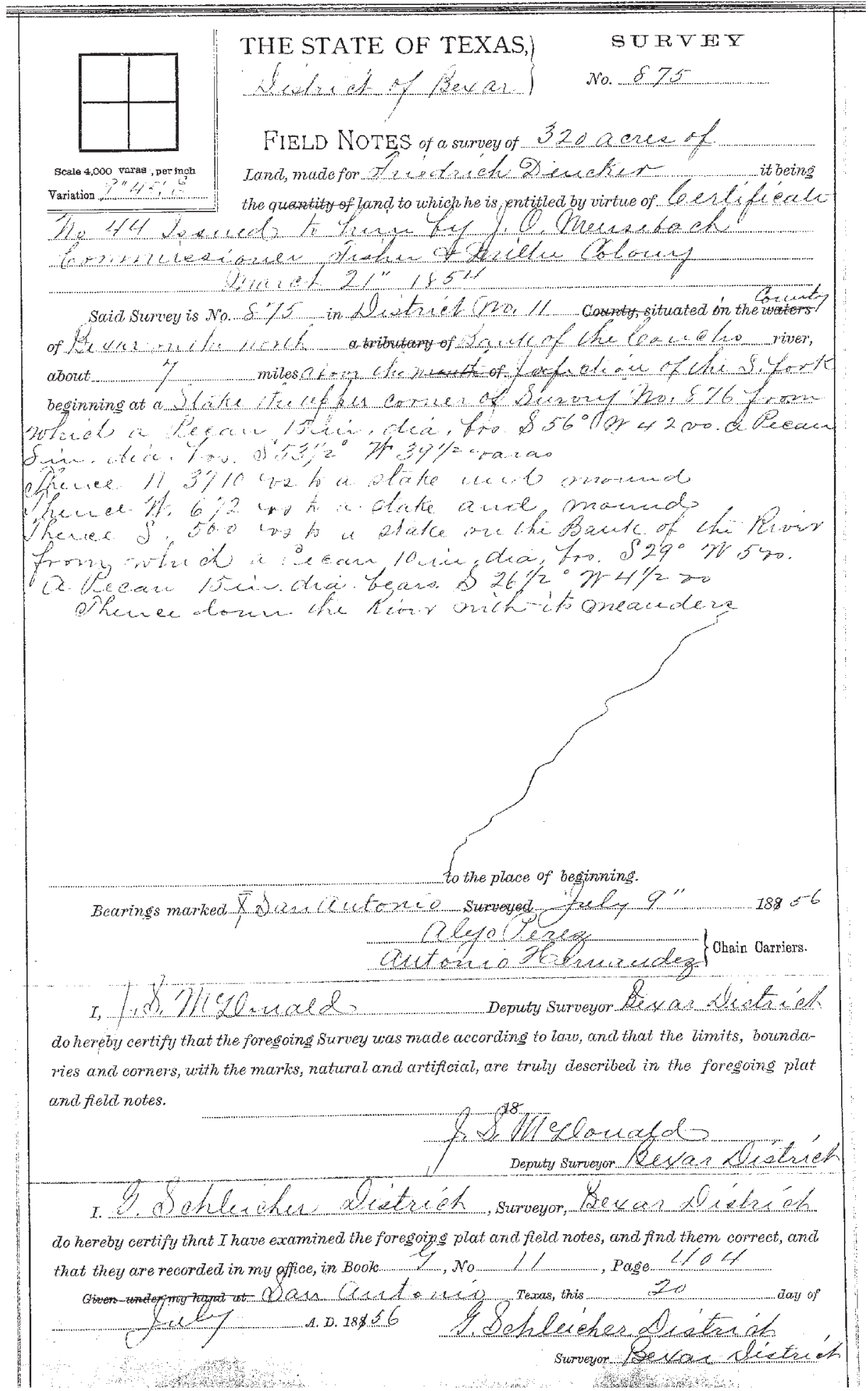

Figure L-9. Survey \#875 (41TG458), Surveyor's Record, Book 2, page 316. West Texas Collection, Angelo State University. 


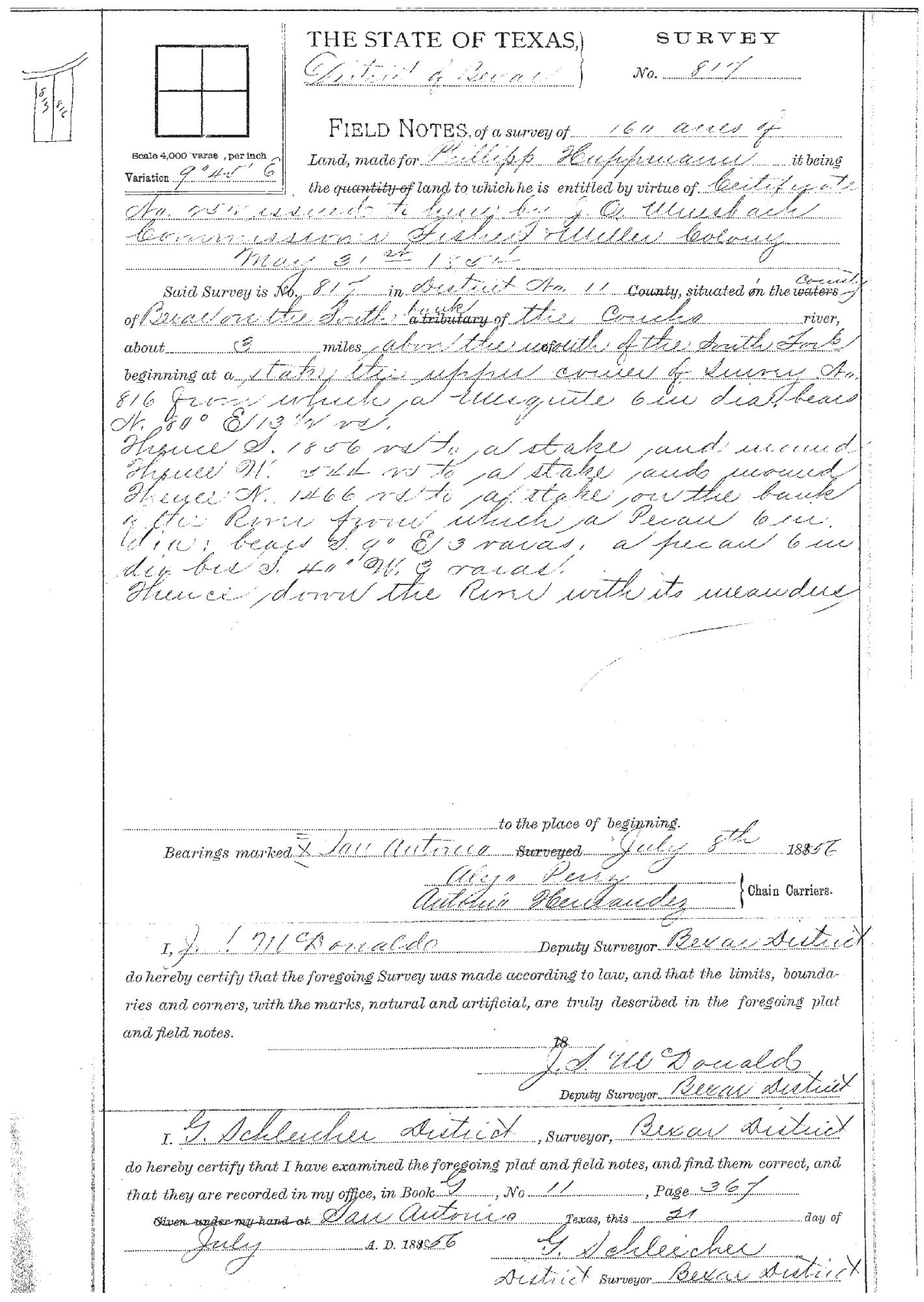

Figure L-10. Survey \#817 (41TG501), Surveyor's Record. West Texas Collection, Angelo State University. 


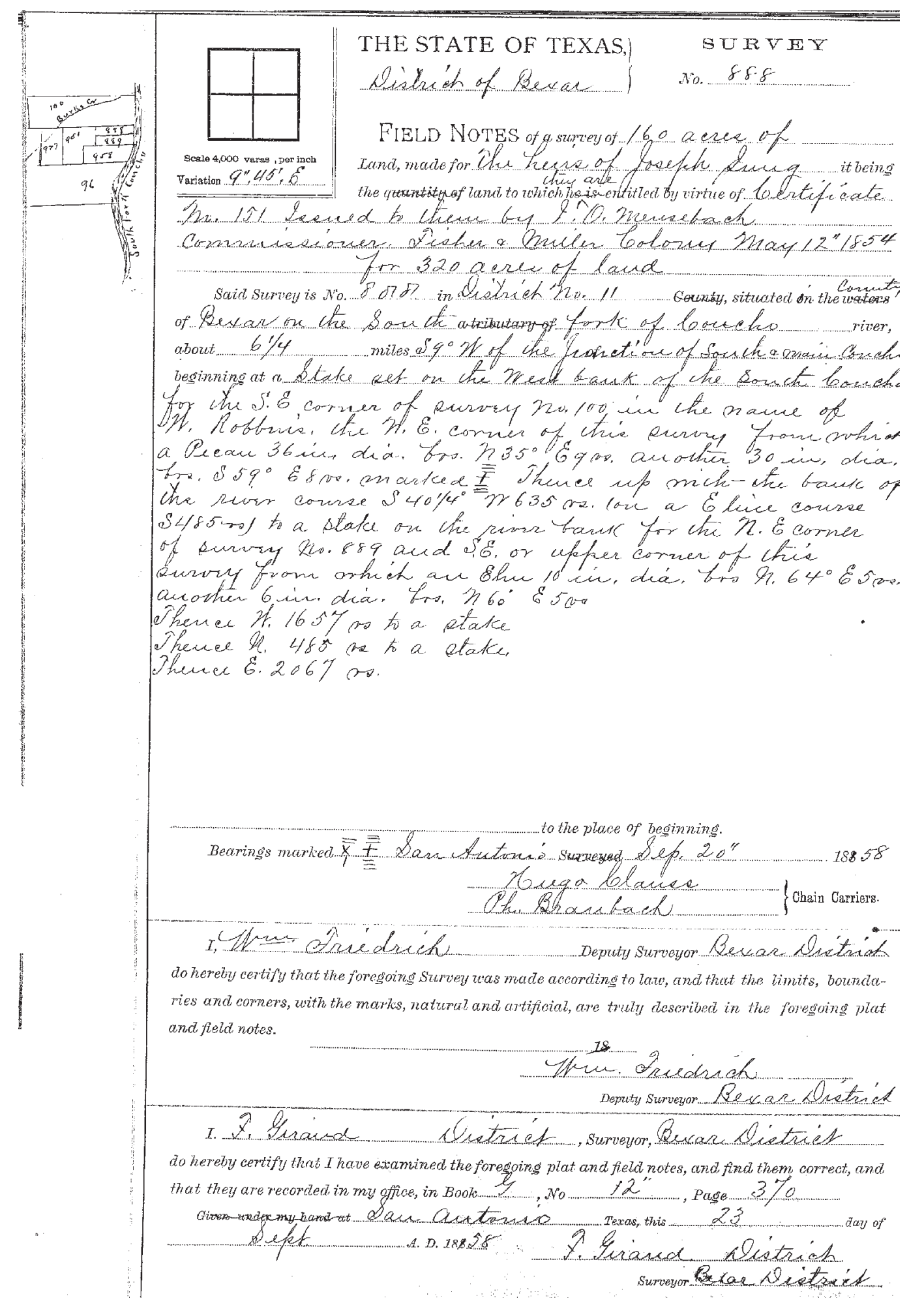

Figure L-11. Survey \#888 (41TG504), Surveyor's Record. West Texas Collection, Angelo State University. 


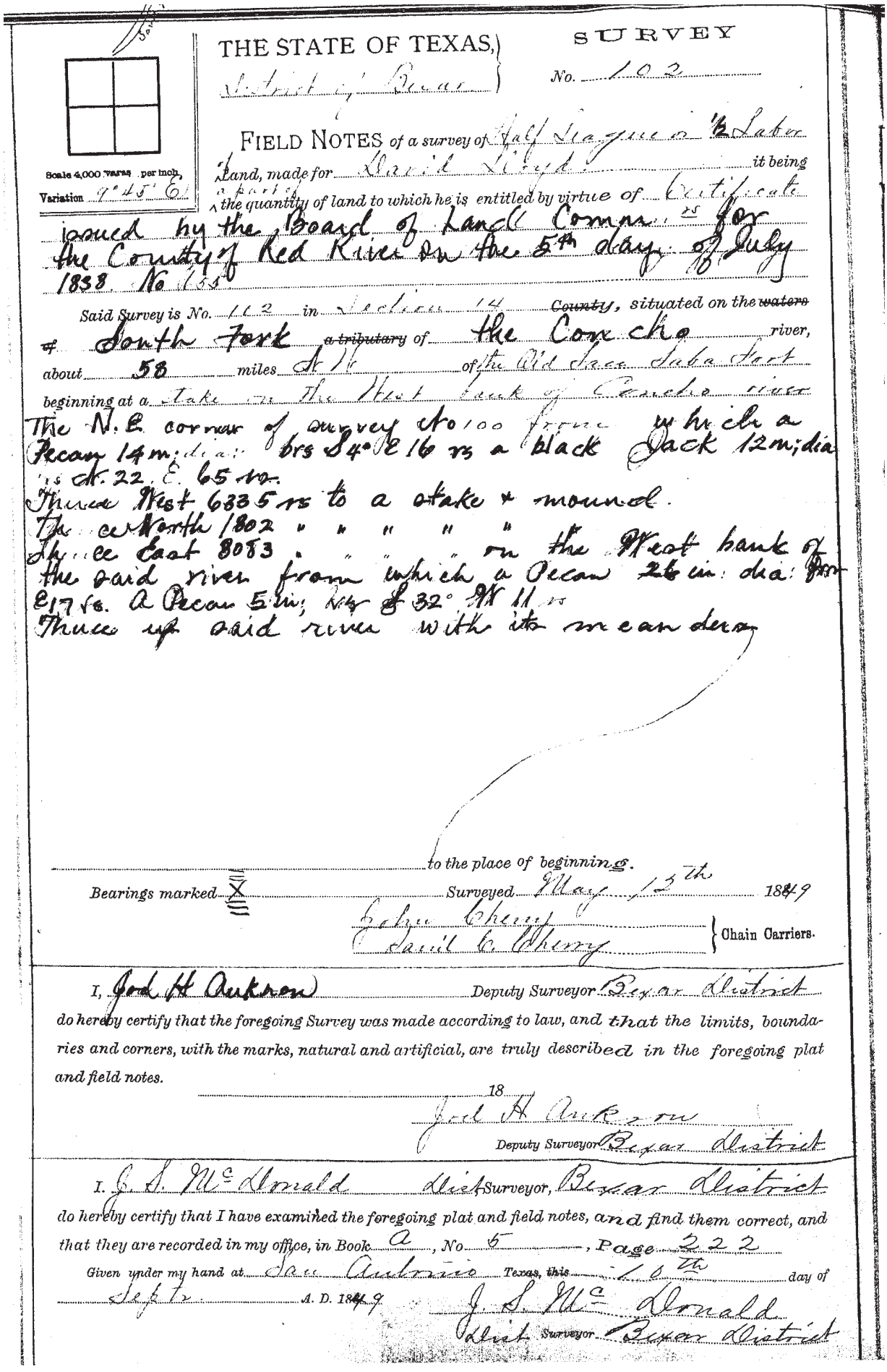

Figure L-12. Survey \#102 (41TG513), Surveyor's Record. West Texas Collection, Angelo State University. 


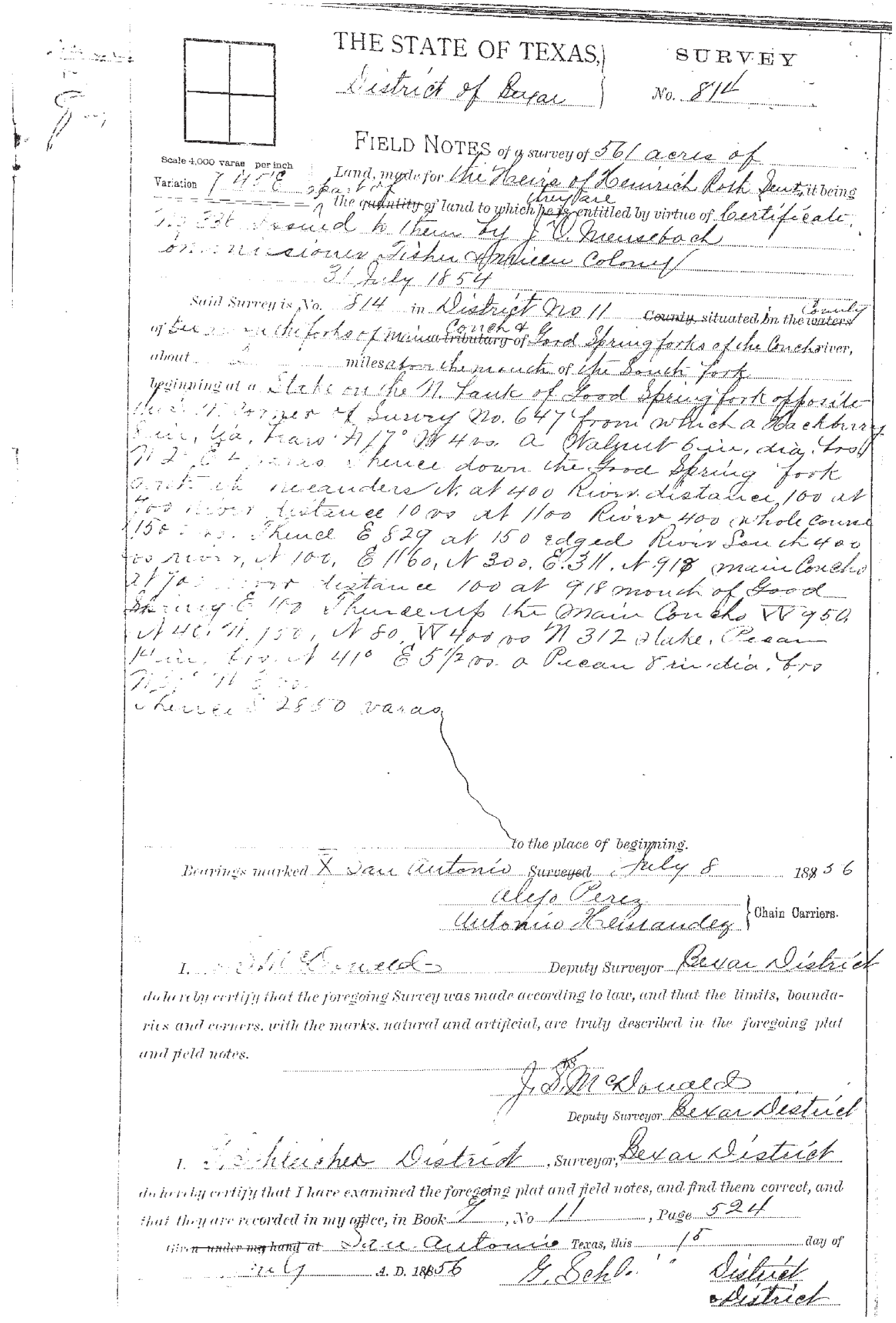

Figure L-13. Survey \#814 (41TG516), Surveyor's Record, Book 2, page 341. West Texas Collection, Angelo State University. 


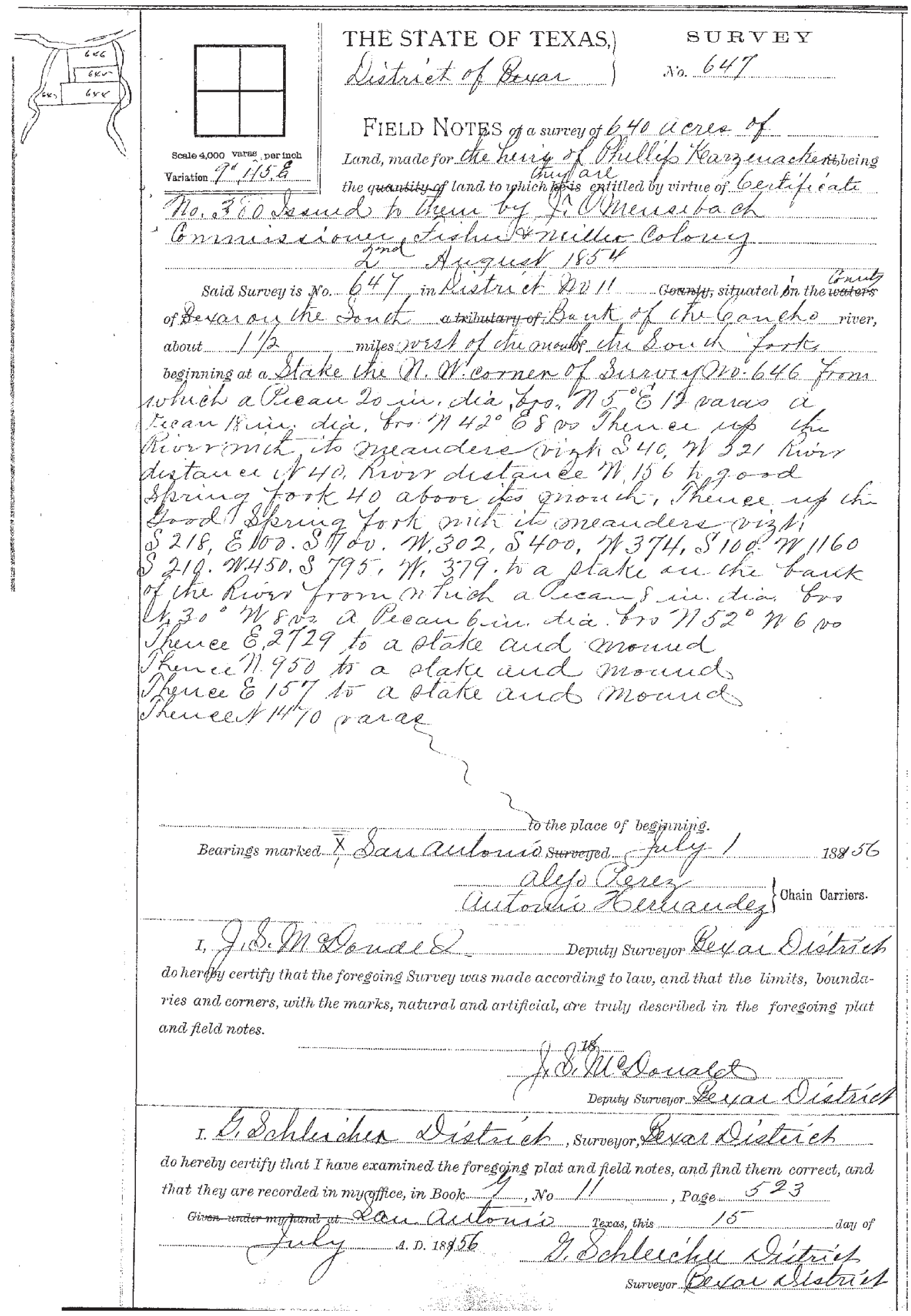

Figure L-14. Survey \#647 (41TG520, 41TG521, and 41TG523), Surveyor's Record, Book 2, page 340. West Texas Collection, Angelo State University. 